UNIVERSIDAD NACIONAL DE LA PLATA

FACULTAD DE CIENCIAS EXACTAS

Departamento de Química

\title{
Propiedades fotofísicas y reactividad fotoquímica de especies poliméricas derivatizadas con grupos cromóforos del tipo $-\operatorname{Re}(\mathrm{CO})_{3} \mathrm{~L}(\mathrm{~L}=$ Azina)
}

\section{Larisa L. B. Bracco}

\author{
Cinética y fotoquímica en solución \\ Instituto de Investigaciones Fisicoquímicas Teóricas y Aplicadas \\ Directores de tesis: Prof. Dr. Mario R. Féliz y Prof. Dr. Ezequiel Wolcan
}


El presente trabajo de tesis se realizó en el Instituto de Investigaciones Fisicoquímicas Teóricas y Aplicadas bajo la dirección del Dr. Mario R. Féliz y del Dr. Ezequiel Wolcan. Se presenta a consideración de las autoridades de la Facultad de Ciencias Exactas de la Universidad Nacional de La Plata a fin de acceder al grado Académico de Doctor de la Facultad de Ciencias Exactas. 
Por medio de las siguientes líneas deseo expresar mi agradecimiento a todas aquellas personas e instituciones que directa o indirectamente han contribuido a la realización de esta tesis doctoral:

Al Prof. Dr. Mario R Féliz, por dirigirme, permitirme trabajar en libertad y darme la posibilidad de aprender de su experiencia.

Al Prof. Dr. Ezequiel Wolcan, porque no sólo me co-dirigió, sino que guió cada uno de mis pasos, estando al lado mío en cada experimento, desde la disolución de las muestras hasta el análisis de datos, porque si no hubiera sido por su guía esto no hubiera sido posible.

Al Dr. Guillermo Ferraudi, por permitir que parte de los experimentos se realicen en el Radiation Laboratory, Universidad de Notre Dame, Indiana, Estados Unidos.

A la Dra. M. Paula Juliarena y al Dr. Gustavo Ruiz, porque me recibieron en el grupo con la mejor predisposición, por ayudarme, por las medidas que hicieron en EE. UU. Por los mates, por las charlas, por los consejos...

Al Ing. Ulises Fagioli y a la Dra Yeny Tobón, por los momentos compartidos.

A mis compañeros del primer piso, gracias a TODOS, becarios e investigadores que día a día compartieron conmigo este camino.

A mis compañeros de la cátedra de química inorgánica de la Facultad de Ciencias Exactas, UNLP, porque además de compartir la difícil tarea de "ayudar a estudiar" me ayudan con su buen humor y alegría a disfrutar mi trabajo, gracias Mica, Pau, Euge, Coqui, Carlitos, Darío, Matías, Lucas, Mauricio, Andrés, Patricia, Mario, Marta y Ale.

A la Dra. Susana Cortizo y el grupo de macromoléculas, por estar siempre dispuestos a ayudarme y prestarme sus instalaciones para poder centrifugar, agitar y medir viscosidad. 
Al Turco Caram y el grupo de Fisicoquímica Orgánica, por permitirme trabajar en su laboratorio con total libertad, utilizando el sonicador y la cámara seca.

A la Agencia, ANPCyT por la beca de iniciación.

Al CONICET, por la beca de postgrado que he recibido para realizar el presente trabajo

A mi madre, por enseñarme que la fuerza de voluntad vale la pena, por educarme, por existir.

A mis hermanos, por ayudarme siempre.

A los que ya no están, en especial a mi padre que aún con su temprana partida supo dejarme su ejemplo de buena persona, a mi abuela Ani, porque me cuidó, estuvo al lado nuestro incondicionalmente y porque estaba llena de humildad, a mi madrina Gladys, porque me brindó su amor en todo momento.

A mis hijitos, Matu y Bauti, porque llenan de alegría mis días.

A mis amigos, que estuvieron SIEMPRE, Vir, Milvia, Lore, Piojo, Chiqui, Ceci, Lorena R. 
Introducción

Referencias

Parte I. Complejos $\mathrm{d}^{6} \mathrm{y}$ métodos experimentales

Capítulo I: Complejos $d^{6}$ en entorno octaédrico 6

Capítulo II: Espectros de absorción $\quad 28$

Capítulo III: Espectroscopía de emisión estacionaria 30

Capítulo IV: Láser flash fotólisis 36

$\begin{array}{ll}\text { Capítulo V: Radiólisis de pulso } & 47\end{array}$

$\begin{array}{ll}\text { Bibliografia } & 54\end{array}$

Parte II: Polímeros derivatizados con complejos del tipo $\left.\operatorname{Re}(\mathrm{CO})_{3} \mathrm{~L} ; \mathrm{L}=\mathrm{Azina}\right)$

Capítulo I: Síntesis y caracterización polímeros de $\mathrm{Re}^{\mathrm{I}} \quad 56$

Capítulo II: Transferencia de energía en polímeros de $\operatorname{Re}^{\mathrm{I}} \quad 74$

Capítulo III: Reducción fotoinducida de Citocromo c por un polímero de $\operatorname{Re}^{\mathrm{I}} \quad 122$

Capítulo IV: Reacción bimolecular de transferencia de electrones entre un

Polímero de $\operatorname{Re}^{\mathrm{I}} \mathrm{y}$ aminas $\mathrm{y}$ un monómero de $\operatorname{Re}^{\mathrm{l}} \mathrm{y}$ aminas $\quad 150$ 


\section{Introducción}

Las propiedades fotoquímicas y fotofísicas de complejos tricarbonílicos de $\operatorname{Re}^{\mathrm{l}} \mathrm{del}$ tipo $\mathrm{XRe}(\mathrm{CO})_{3} \mathrm{~L}_{2}$ donde $\mathrm{X}=$ haluro, azina o fosfina; $\mathrm{L}=$ Azina monodentada o $\mathrm{L}_{2}$ : Azina bidentada, han sido objeto de numerosas investigaciones desde mediados de la década del '70 cuando se pudieron describir por primera vez las propiedades de los estados excitados en algunos compuestos de esta familia ${ }^{1-30}$. Sin embargo, las investigaciones en este campo, continúan siendo de gran interés por la potencial aplicación de estos compuestos en fotocatálisis $^{4,7-8,15,22,24,28}$, como sensores luminiscentes, en la derivatización de polímeros fotoreactivos, como sensibilizadores semiconductores, en sondas específicas para biomoléculas, como inductores de fotoclivaje de ADN etc. ${ }^{29,31,32}$

En los últimos años se han realizado numerosos estudios en polímeros derivatizados con este tipo de complejos. Se han estudiado por ejemplo, las propiedades fotofísicas y fotoquímicas de la polivinilpiridina, (vpy $)_{600}$ derivatizada con el complejo $-\operatorname{Re}(\mathrm{CO})_{3}(1,10-$ phen $)^{+}$, donde 1,10 -phen $=1,10$-Fenantrolina, de manera que cada molécula de polímero posee 200 cromóforos que penden del esqueleto de la polivinilpiridina. Se han comparado las propiedades fotofísicas y fotoquímicas de este polímero con su respectivo monómero. ${ }^{33}$ La principal causa de las diferencias encontradas en dichas propiedades, es la fotogeneración de estados excitados de transferencia de carga del $\mathrm{Re}^{\mathrm{l}}$ al ligando, TCML, en concentraciones que son mucho mayores cuando los cromóforos $-\operatorname{Re}(\mathrm{CO})_{3} \mathrm{~L}^{+}$están coordinados al esqueleto del polímero que cuando se hallan distribuidos en soluciones diluidas de los monómeros. Este es el resultado fotofísico de disponer de cromóforos de $\mathrm{Re}^{\mathrm{I}}$ agrupados en pequeñas regiones de las cadenas del polímero en lugar de hallarse 
homogéneamente distribuidos en la solución del monómero. También se ha visto la asociación de varios cientos de cadenas poliméricas en agregados casi esféricos. ${ }^{\mathbf{3 4}}$

Además pueden observarse efectos del medio debido a la naturaleza polielectrolítica de los polímeros, hecho que puede afectar las reacciones de estados excitados. Algunos de estos efectos fueron previamente estudiados en la fotoquímica de complejos de $\mathrm{Cu}$ (II) que penden de cadenas de poliacrilatos. ${ }^{35}$

El objetivo de este trabajo de tesis es el estudio fotofísico y fotoquímico de nuevos complejos del tipo $-\operatorname{Re}^{\mathrm{I}}(\mathrm{CO})_{3} \mathrm{~L}_{2}$ que penden de un esqueleto de polivinilpiridina.

En la Parte I se describe en primer lugar la configuración electrónica de complejos $d^{6}$ en un entorno octaédrico, familia a la que pertenecen los complejos tricarbonílicos de $\operatorname{Re}^{\mathrm{I}}$. Luego se describe la generalidad de los procesos fotofísicos y fotoquímicos. Por último se realiza una descripción de los métodos experimentales utilizados para el estudio de estos procesos, como espectroscopia de emisión y absorción estacionaria y resuelta en el tiempo, y radiólisis de pulso.

En la Parte II, se detalla la síntesis y caracterización de los polímeros:

$\left\{(\text { vpy })_{2} \text { vpyRe }(\mathrm{CO})_{3}\left(\mathrm{NO}_{2}-\text { phen }\right)^{+}\right\}_{\mathrm{m} \sim 200} ;\left\{(\mathrm{vpy})_{2} \mathrm{vpyRe}(\mathrm{CO})_{3}(\text { tmephen })^{+}\right\}_{\mathrm{n} \sim 200} ;$

$\left\{(\text { vpy })_{2} \text { vpyRe }(\mathrm{CO})_{3}(\text { tmephen })^{+}\right\}_{\mathrm{n} \sim 180}\left\{(\mathrm{vpy})_{2} \operatorname{vpyRe}(\mathrm{CO})_{3}\left(\mathrm{NO}_{2}-\text { phen }\right)^{+}\right\}_{\mathrm{m} \sim 20}$;

$\left\{(\text { vpy })_{2} \operatorname{vpyRe}(\mathrm{CO})_{3}(\text { tmephen })^{+}\right\}_{n \sim 150}\left\{(\text { vpy })_{2} \operatorname{vpyRe}(\mathrm{CO})_{3}\left(\mathrm{NO}_{2}-\text { phen }\right)^{+}\right\}_{\mathrm{m} \sim 50} \mathrm{y}$

$\left\{(\text { vpy })_{2} \text { vpyRe }(\mathrm{CO})_{3}(\text { tmephen })^{+}\right)_{\mathrm{n} \sim 100}\left\{(\text { vpy })_{2} \text { vpyRe}(\mathrm{CO})_{3}\left(\mathrm{NO}_{2}-\text { phen }\right)^{+}\right\}_{\mathrm{m} \sim 100}$

donde vpy; vinilpiridina; 5-NO2-phen: 5-Nitro;1,10-Fenantrolina y

tmphen: 3.4.7.8 tetrametil-1,10-Fenantrolina.

Se muestran los resultados de espectroscopía de absorción y emisión estacionaria y resuelta en el tiempo, y se describe el proceso de transferencia de energía observado en los polímeros mixtos: 
$\left\{(\text { vpy })_{2} \text { vpyRe }(\mathrm{CO})_{3}(\text { tmephen })^{+}\right\}_{\mathrm{n} \sim 180}\left\{(\mathrm{vpy})_{2} \mathrm{vpyRe}(\mathrm{CO})_{3}\left(\mathrm{NO}_{2}-\text { phen }\right)^{+}\right\}_{\mathrm{m} \sim 20} ;$

$\left\{(\text { vpy })_{2} \text { vpyRe }(\mathrm{CO})_{3}(\text { tmephen })^{+}\right\}_{n \sim 150}\left\{(\text { vpy })_{2} \text { vpyRe }(\mathrm{CO})_{3}\left(\mathrm{NO}_{2}-\text { phen }\right)^{+}\right\}_{\mathbf{m} \sim 50} \mathrm{y}$

$\left\{(\text { vpy })_{2} \operatorname{vpyRe}(\mathrm{CO})_{3}(\text { tmephen })^{+}\right)_{\mathrm{n} \sim 100}\left\{(\mathrm{vpy})_{2} \operatorname{vpyRe}(\mathrm{CO})_{3}\left(\mathrm{NO}_{2}-\text { phen }\right)^{+}\right\}_{\mathrm{m} \sim 100}$

Se presentan los resultados de reducción fotoinducida de Citocromo c por el estado excitado del polímero $\left\{(\mathrm{vpy})_{2} \mathrm{vpyRe}(\mathrm{CO})_{3}(\text { tmephen })^{+}\right\}_{\mathrm{m} \sim 200}$.

Y por último se estudia el proceso de desactivación (quenching) del estado excitado de transferencia de carga metal-ligando, TCML, del monómero $\operatorname{PyRe}(\mathrm{CO})_{3}\left(2,2^{\prime} \mathrm{Bpy}\right)$ y del polimero $\left\{(\mathrm{vpy})_{2} \mathrm{vpy} \operatorname{Re}(\mathrm{CO})_{3}\left(2,2^{\prime} \mathrm{Bpy}\right)^{+}\right\}_{\mathrm{m} \sim 200}$, donde Py: Piridina y $2,2^{\prime} \mathrm{Bpy}$; 2,2'Bipiridina; con una serie de aminas alifáticas y aromáticas. Este proceso como se verá mas adelante es un proceso redox en donde ocurre la oxidación de la amina y la reducción del estado excitado de TCML. Esta serie de aminas se eligió de modo de disponer de un amplio rango de potenciales de oxidación. De esta manera se varía ampliamente el $\Delta \mathrm{G}$ de la reacción. El trabajo apunta a establecer las diferencias entre el comportamiento del monómero y el polímero frente al proceso de desactivación reductiva. 


\section{Referencias}

1. Wrighton, M.S.; Morse, D.L. J. Am. Chem. Soc. 1974, 96, 998.

2. Luong, J.C.; Faltynak, H.; Wrighton, M.S. J. Am. Chem. Soc. 1979, 101, 1597.

3. Giordano, P.J.; Fredericks, S.M.; Wrighton, M.S.; Morse, D.L. J. Am. Chem. Soc. 1978, 100, 2257.

4. Luong, J.C.; Nadjo, L.; Wrighton, M.S. J. Am. Chem. Soc. 1978, 100, 5790.

5. Giordano; P.J.; Wrighton, M.S. J. Am. Chem. Soc. 1979, 101, 2888.

6. Fredericks, S.M.; Luong, J.C.; Wrighton, M.S. J. Am. Chem. Soc. 1979, $101,7415$.

7. Summers, D.P.; Luong, J.C.; Wrighton, M.S. J. Am. Chem. Soc. 1981, 103, 5238.

8. Shu, C.; Wrighton, M.S. Inorg. Chem. 1988, 27, 4326.

9. Sacksteder, L.; Zipp, A.P.; Brown, E.A.; Streich, J.; Demas, J.N.; Degraff, B.A. Inorg.Chem. 1990, 29, 4335.

10. Perkins, T.A.; Humer, W.; Netzel, T.L.; Schanze, K.S. J. Phys. Chem. 1990, 94, 2229.

11. Shaw, J.R.; Schmehl, R.H.; J. Am. Chem. Soc. 1991, 113, 389.

12. Glezen, M.M.; Lees, A.J. J. Am. Chem. Commun. 1987, 1752.

13. Glezen, M.M.; Lees, A.J. J. Am. Chem. Soc. 1988, 110, 3892.

14. Glezen, M.M.; Lees, A.J. J. Am. Chem. Soc. 1988, 110, 6243.

15. Hawecker, J.; Lehn, J.M.; Ziessel, R.; J. Chem. Soc., Chem. Commun. 1984, 328.

16. Kutal, C; Weber, M.A.; Ferraudi, G.; Geiger, D. Organometallics 1985, 4, 2161.

17. Kutal, C.; Corbin, A.J.; Ferraudi, G.; Geiger, D. Organometallics 1987, 6, 553.

18. Féliz, M.R.; Ferraudi, G. Chem. Phys. Lett. 1991, 181, 201.

19. Caspar, J.V.; Meyer, T.J. J. Phys. Chem. 1983, 87, 952.

20. Sullivan, B.P.; Meyer, T.J. J. Chem. Soc., Chem. Commun. 1984, 1244.

21. Caspar, J.V.; Sullivan, B.P.; Meyer, T.J. Inorg. Chem. 1984, 23, 2104. 
22. Westmoreland, T.D.; Schanze, K.S.; Neveux, Jr., P.E.; Danielson, E.; Sullivan, B.P.;Chen, P.; Meyer, T.J. Inorg. Chem. 1985, 24, 2596.

23. G. Ferraudi, Neyde Murakami Iha, G. Ruiz, E. Wolcan and M.R.Féliz, Inorg. Chim.Acta, 221, 1994, 161-164

24. G.Ruiz, E.Wolcan, A.L.Capparelli and M.R.Féliz, J. Photochem. Photobiol. A: Chem.89, 1995, 61-66

25. G. Ruiz, E. Wolcan and M. R. Féliz, J. Photochem. Photobiol. A: Chem. 101, $1996,119-125$

26. G. Ruiz, F. Rodriguez Nieto, E. Wolcan and M. R. Féliz, J. Photochem. Photobiol. A:Chem. 107, 1997, 47-54

27. M. R. Féliz, F. Rodriguez Nieto, G. Ruiz and E. Wolcan, J. Photochem. Photobiol. A:Chem. 117, 1998, 185-192.

28. Kalyanasundaram K. and Grätzel M. "Photosensitization and Photocatalysis UsingInorganic and Organometallic Compounds" Kluwer Academic Publishers, Dordrecht, 1993.

29. Stufkens D.J. and Vlèek Jr A. Coord. Chem. Rev. 177 (1998) 127-179.

30. L.S. Forster, Coord. Chem. Rev. 227, 2002, 59-92

31. Autores varios, "Applications of Inorganic Photochemistry", J. Chem. Educ. 1997, Vol74, Nro.6, 633-702.

32. K.E. Ekkila, D.T. Odom and J.K. Barton, Chem. Rev. 1999, 99, 2777-2795.

33. E. Wolcan; G. Ferraudi; J. of Phys. Chem. A. 2000. 104, 9281

34. Wolcan, E; Alessandrini, J. L.; Félia, M. R. J. Phys. Chem. B 2005, 109, 22890

35. Baumgartner, E; Ronco, S; Ferraudi, G;. Inorg. Chem. 1990. 29, 1329 


\section{Parte I}

Complejos $d^{6}$ en entorno octaédrico y métodos experimentales 
Capítulo I

Complejos $d^{6}$ en entorno octaédrico 


\section{Capítulo I}

\section{Complejos de metales de transición con configuración electrónica d}

\section{i. Electrones en el metal}

Los complejos de $\operatorname{Re}$ en estado de oxidación +1 presentan una configuración electrónica en la cual los orbitales $5 d$ poseen 6 electrones apareados, es decir $d^{6}$ de bajo spín.

La teoría del campo cristalino (TCC), expuesta por primera vez por $\mathrm{H}$. Bethe en 1929, trata al ión metálico central en un complejo (o en un medio cristalino) como si estuviera sometido por sus ligantes o vecinos más cercanos a una perturbación puramente electrostática; los ligantes o vecinos se tratan simplemente como si fueran cargas de punto o dipolos de punto. La teoría del campo de ligantes (TCL) introduce algunas modificaciones ad hoc, dictadas empíricamente, que compensan en forma parcial la naturaleza física irreal del modelo TCC.

A pesar de la extrema simplicidad y naturaleza no física de ambas teorías, estas proporcionan resultados provechosos, debido al uso que hacen de las propiedades de simetría, tanto de la estructura como de los orbitales metálicos.

La idea central de la TCC y la TCL, es que la pentadegeneración de los orbitales d desaparece cuando un átomo metálico está rodeado por un conjunto de cargas negativas o de los extremos negativos de los dipolos, figura 1. Los patrones de la energía producida dependen de la simetría en la distribución de los ligantes. 
Considerando un complejo octaédrico, $\mathrm{ML}_{6}$, en el que los ligantes $\mathrm{L}$ son aniones que se tratan como cargas eléctricas distribuidas como en la figura 2 y al observar la figura 3 que muestra la distribución espacial de la carga electrónica en los orbitales d del átomo metálico $\mathrm{M}$; es evidente que los orbitales $\mathrm{d}_{\mathrm{xy}}, \mathrm{d}_{\mathrm{yz}}, \mathrm{y} \mathrm{d}_{\mathrm{zx}}$ son equivalentes y colocan la densidad electrónica entre los ejes $\mathrm{M}-\mathrm{L}$, mientras que en los orbitales $\mathrm{d}_{\mathrm{z} 2}$ y $\mathrm{d}_{\mathrm{x} 2-\mathrm{y} 2}$ la densidad electrónica se dirige hacia los ligantes negativos. Es posible demostrar, aunque no es obvio que los últimos dos orbitales son equivalentes. El efecto de los seis ligantes negativamente cargados es el de dividir los orbitales $\mathrm{d}$ en dos conjuntos, uno triplemente degenerado y el otro doblemente degenerado, los primeros son mas estables y la diferencia de energía se puede denotar por $\Delta o$. 


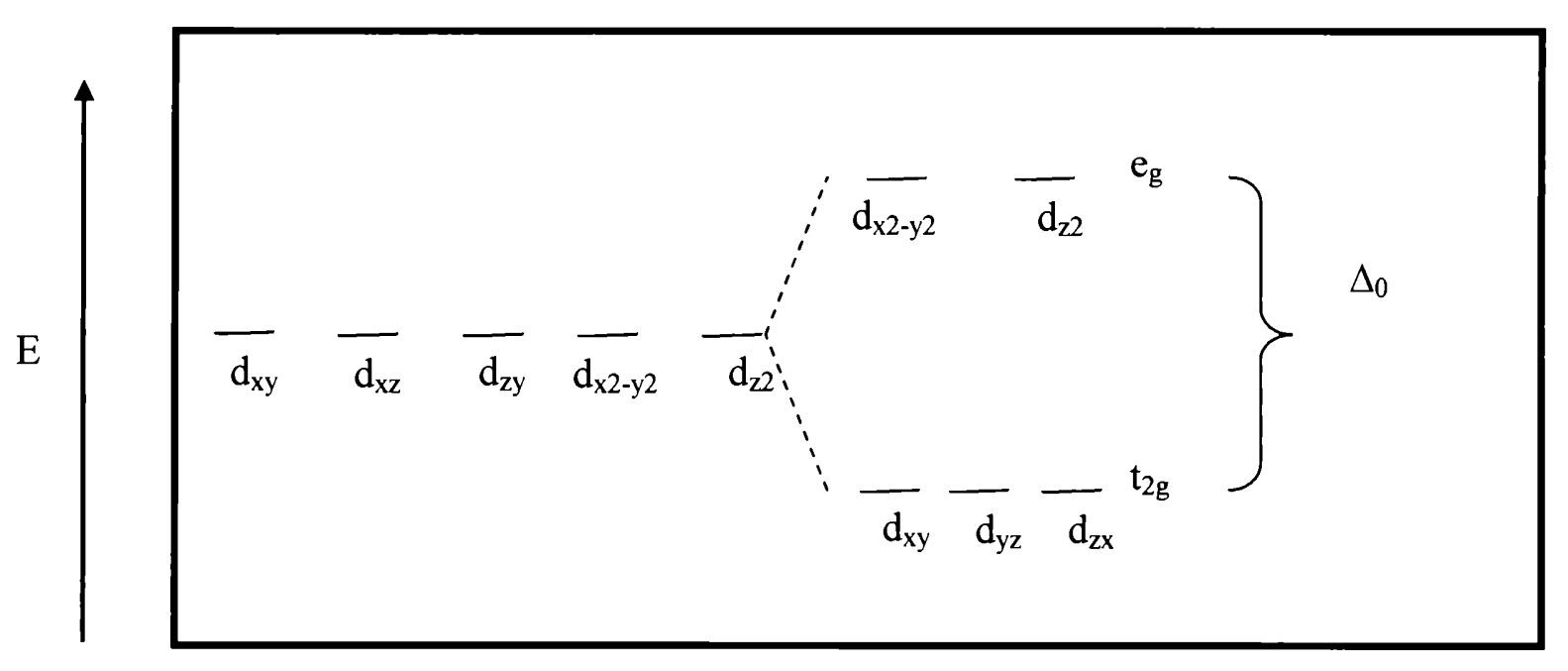

Figura 1: Esquema del desdoblamiento de orbitales $d$ en un entorno octaédrivo

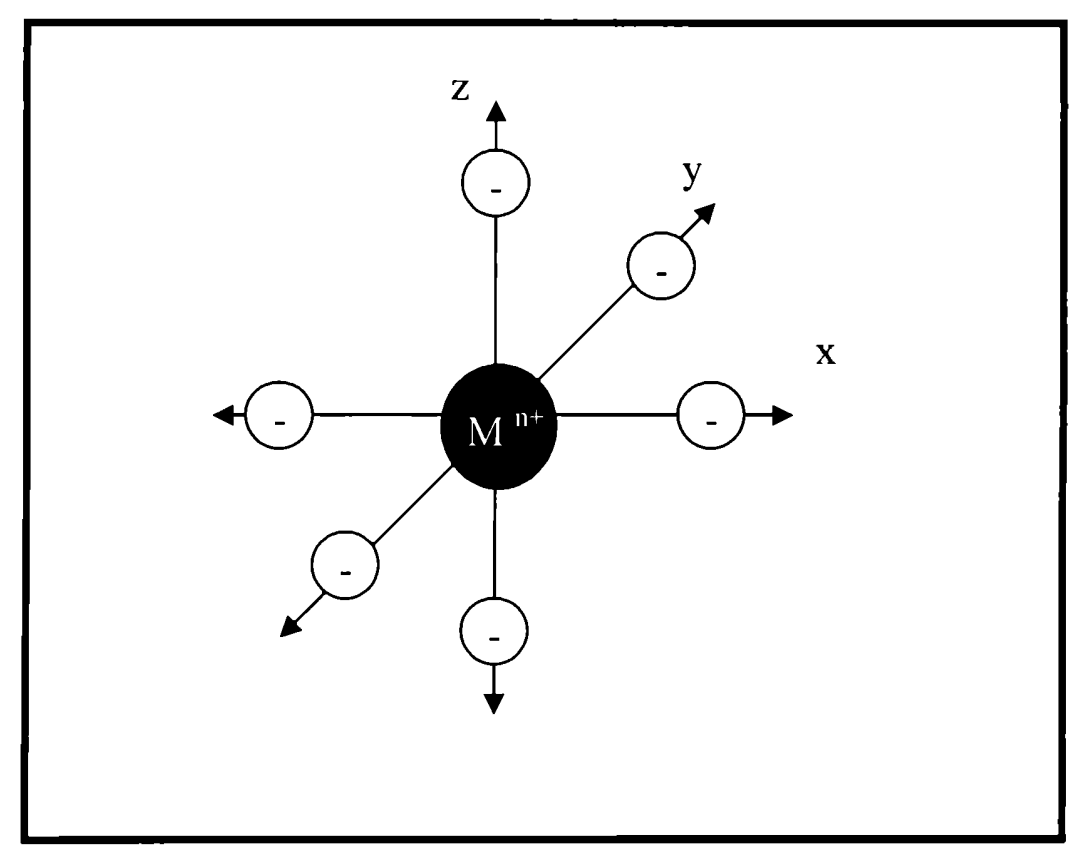

Figura 2: Representación esquemática del complejo octaédrico $\mathrm{ML}_{6}$. 


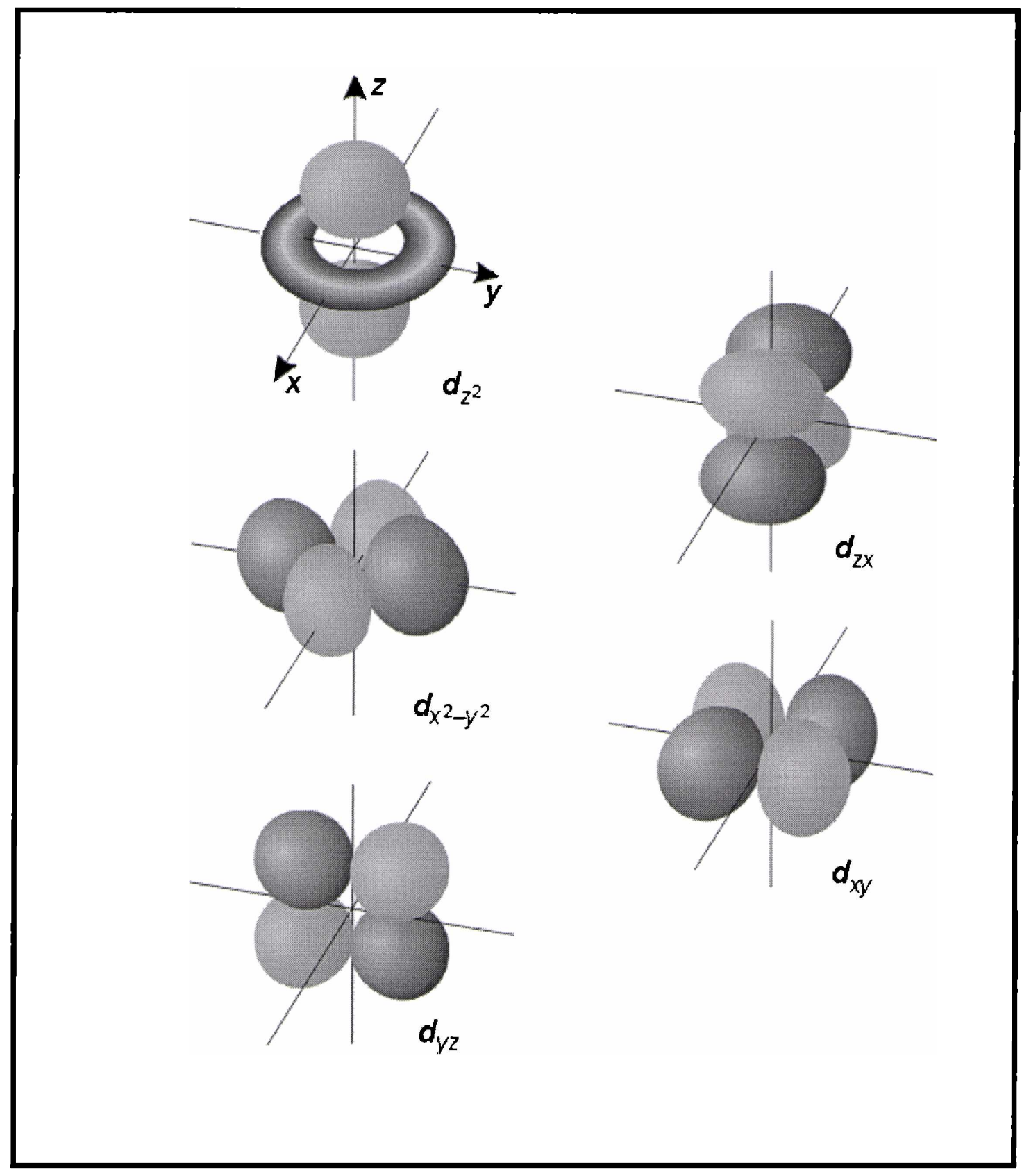

Figura 3: Representación espacial de los orbitales d

El valor de $\Delta \mathrm{o}$ es dependiente de la carga del metal central, la serie de transición a la que pertenece y del ligando que forma el complejo (serie espectroscópica). 
Para el caso de complejos de $\mathrm{Re}^{\mathrm{I}}$ todos presentan una configuración $\mathrm{d}^{6}$ de bajo spín, debido a que la energía necesaria para aparear los electrones en una configuración de bajo spín es favorable con respecto al valor de $\Delta \mathrm{o}$.

\section{ii. Electrones en el ligando}

En una gran cantidad de complejos, los ligandos en su forma libre presentan electrones en orbitales no enlazantes que son fácilmente excitables. La molécula de piridina, un ligando común, presenta un par de electrones en orbitales de no enlace $\left(\mathrm{n}^{2}\right)$ en el átomo de nitrógeno, que pueden ser excitados a un orbital de antienlace sobre el anillo aromático $\left(\pi^{*}\right)$.

La primera transición $n \rightarrow \pi^{*}$, para la piridina es la transición de mas baja energía, seguida por la transición $\pi \rightarrow \pi^{*}$, dentro del anillo aromático, figura 4 .

Cuando la piridina coordina con un ión metálico los electrones $\mathrm{n}$ sobre el $\mathrm{N}$ forman una unión $\sigma$ con el ión, con lo cual, estos electrones no pueden ser excitados por luz de baja energia. Las transiciones $\pi \rightarrow \pi^{*}$, dentro del anillo aromático, en general, no son afectadas por la complejación y usualmente caen en la misma región que en el ligando libre, figura 4. 


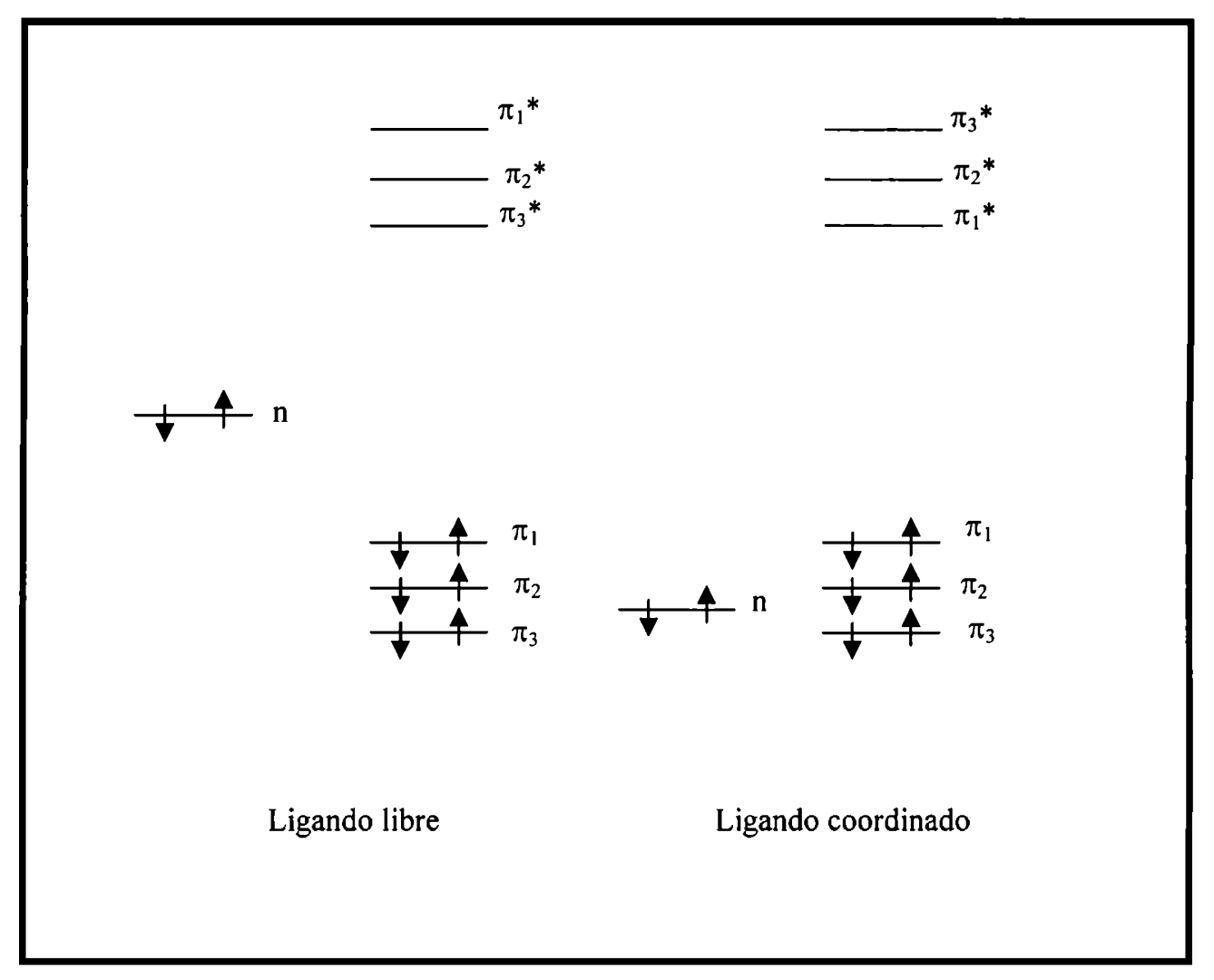

Figura 4: Representación esquemática de orbitales de ligandos N-heterocíclicos.

\section{iii. Electrones en un complejo $d^{6}$}

La interesante fotoquímica y espectroscopia de complejos de $\mathrm{Rh}(\mathrm{III}), \mathrm{Co}(\mathrm{III})$, $\operatorname{Ru}(\mathrm{II}), \operatorname{Re}(\mathrm{I})$, entre otros, reside en los diferentes estados excitados de baja energía que presentan y que son accesibles con luz visibles y radiación UV cercana.

Suponiendo que el campo producido por presencia del ligando es fuerte, el estado fundamental tendrá una configuración $\left(\mathrm{t}_{2 \mathrm{~g}}\right)^{6}$, esto es asi para complejos de metales de la segunda y tercera serie de transición. 
De acuerdo al tipo de metal y ligando que forman el complejo es posible que los orbitales adquieran diferente energía y de dicha disposición dependerá la transición de mas baja energía, este hecho se muestra en la figura 5 .

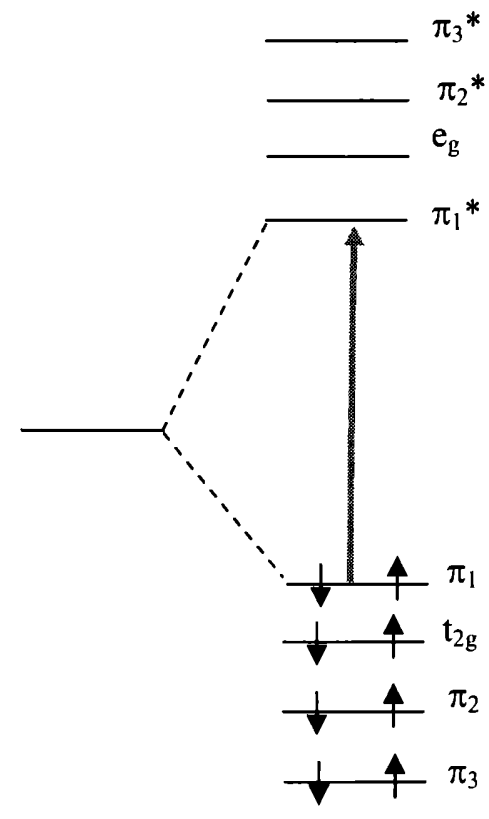

(a)

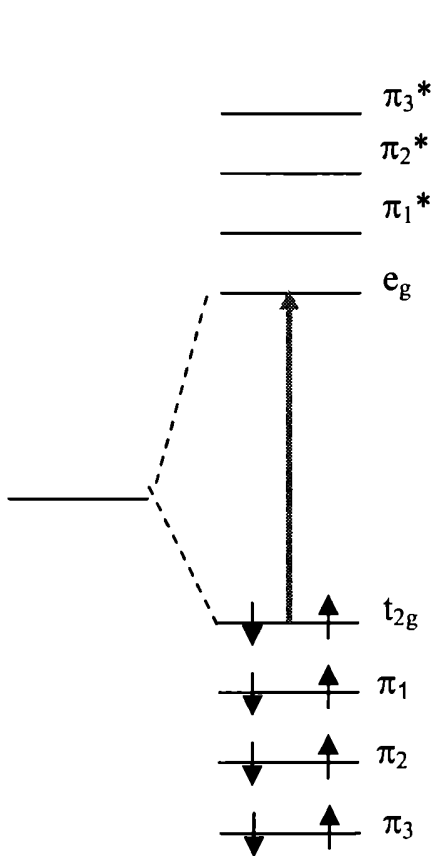

(b)

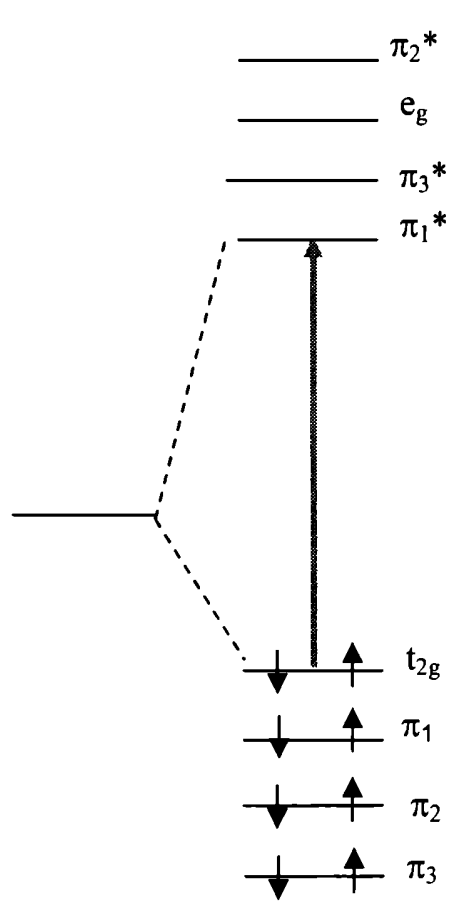

(c)

Figura 5: Representación esquemática de la disposición orbital de un complejo d ${ }^{6}$ en un campo de lignates fuerte. Transición de más baja energía.

En esta figura se pueden apreciar tres casos diferentes de transición de menor energía dependiendo del orden energético en el que se ubiquen los orbitales del metal y del ligando. 
La figura 5.a representa el caso en el que la transición de mas baja energía corresponde a una transición $\pi \rightarrow \pi^{*}$, en este caso los orbitales $t_{2 \mathrm{~g}}$ se llenan antes que los orbitales del ligando, y los orbitales $\mathrm{e}_{\mathrm{g}}$ se encuentran por encima en energía con respecto a los orbitales $\pi^{*}$ del ligando. Por ejemplo, estudios espectroscópicos sobre $\left\{\mathrm{Rh}(\mathrm{bpy})_{3}\right\}^{3+}$, $\left\{\mathrm{Rh}(\text { phen })_{3}\right\}^{3+}$ y otros complejos análogos muestran claramente que el nivel excitado de mas baja energía es derivado de la configuración $\pi \rightarrow \pi^{*}$. Los orbitales del metal no juegan un rol importante en este proceso de excitación, y la transición de energía mas baja está localizada esencialmente en el ligando, por lo cual es de esperar que cualquier proceso químico que ocurra responda a la fotoquímica del ligando.

La figura 5.b representa el caso en el que la transición de mas baja energía corresponde a una transición d-d, en este caso los orbitales $t_{2 g}$ están llenos en el estado fundamental y el orbital desocupado de mas baja energía es el orbital $e_{\mathrm{g}}$, este tipo de transición se da por ejemplo en el complejo $\left\{\mathrm{Co}(\mathrm{CN})_{6}\right\}^{-3}$. La irradiación de soluciones acuosas de $\left.\mathrm{Co}(\mathrm{CN})_{6}\right\}^{-3}$ genera $\left.\mathrm{Co}(\mathrm{CN})_{5}\left(\mathrm{H}_{2} \mathrm{O}\right)\right\}^{-2}$ con alta eficiencia. La fotoquímica sustitucional, es característica en complejos con transiciones $t_{2 g} \rightarrow e_{g}$.

* La figura 5.c representa el caso en el que la transición de mas baja energía corresponde a una transición $\mathrm{d}-\pi^{*}$. Por ejemplo la bipiridina es una molécula no coloreada, el espectro de absorción de este ligando libre muestra que la transición de mas baja energía es una transición $\pi \rightarrow \pi^{*}$. Cuando se adiciona $\mathrm{Ru}(\mathrm{II})$, incoloro, a soluciones acuosas de bipiridina, aparece un color rojo profundo debido a la formación del complejo $\left[\mathrm{Ru}(\mathrm{bpy})_{3}\right]^{2+}$. Las medidas espectrales revelan la aparición de un nuevo set de transiciones electrónicas, que no están localizadas ni en el metal ni en el ligando. Del estudio espectral 
se ha determinado que la intensa transición visible se debe a una transferencia de carga metal ligando donde un electrón sobre el orbital $t_{2 g}$ es promovido a un orbital $\pi^{*}$ sobre el ligando.

iv. Orbitales en complejos tricarbonilicos de $\mathrm{Re}^{I}$

En general, los complejos de metales de transición $d^{6}$ que poseen en su esfera de coordinación ligandos polipiridínicos, presentan estados excitados que se asemejan fuertemente a los observados para el $\mathrm{Ru}\left(2,2^{\prime} \mathrm{Bpy}\right)_{3}{ }^{+2}$. Este, y otros complejos relacionados, han sido ampliamente estudiado y se presentará aquí como complejo "modelo" para entender, de manera simplificada, la dinámica de los estados excitados puestos en juego luego de la excitación electrónica. Los rasgos particulares de complejos tricarbonílicos de $\operatorname{Re}^{1}$ serán comentados convenientemente.

En la figura 6, se ilustra el diagrama de orbitales moleculares resultante de la combinación de los orbitales $4 \mathrm{~d}$ del $\mathrm{Ru}^{\mathrm{II}} \mathrm{y}$ orbitales $n, \pi$ y $\pi *$ de la 2,2 'Bpy. 


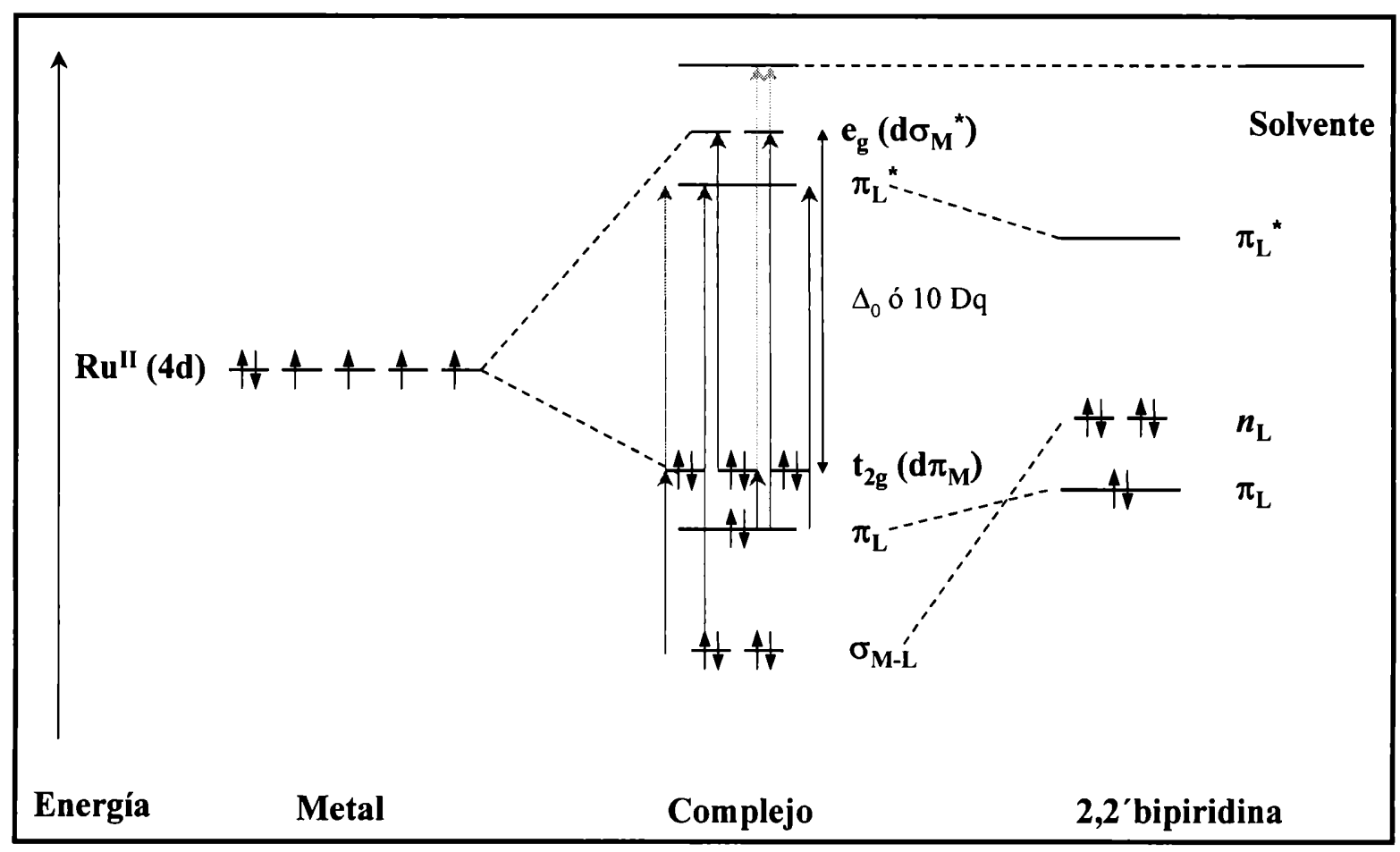

Figura 6: Descripción cualitativa de los orbitales moleculares del Ru(2,2'Bpy $)_{3}{ }^{+2}$ y los tipos de transiciones electrónicas posibles: $(\bullet) \operatorname{TCML},(\bullet) \operatorname{TCLM},(\bullet) \mathrm{IL},(\bullet) \mathrm{CM}$ y (•) TCS.

Los pares de electrones no enlazantes de los átomos de nitrógeno (u orbitales $n$ llenos) participan en la formación de enlaces metal-ligando, estabilizándose y disminuyendo sustancialmente su energía. En función de este diagrama simplificado se pueden identificar las diferentes transiciones electrónicas posibles en esta familia de compuestos luego de la absorción de luz apropiada.

$T C M L$ : la aparición de una intensa absorción en la región visible es un rasgo característico de estos complejos y se corresponde con una transferencia de carga del metal hacia el 
ligando, estas transiciones ocurren cuando un electrón alojado en un orbital localizado sobre el metal es promovido hacia un orbital localizado sobre el ligando $\left(\mathrm{t}_{2 \mathrm{~g}} \rightarrow \pi_{\mathrm{L}}{ }^{*}\right.$

. en la figura 5) presentando típicamente, valores del coeficiente de extinción altos $\left(\varepsilon_{0} \geq 10^{4}\right.$ $\mathrm{M}^{-1} \mathrm{~cm}^{-1}$ ), ya que están permitidas por las reglas de Laporte y usualmente por las del spin. Además se caracterizan por producir separación de cargas, que pueden llevar a reacciones redox.

TCLM: las transiciones en las cuales la carga electrónica es transferida esencialmente desde los ligandos hacia el metal coordinante, pertenecen a otro tipo de transferencia de carga denominada transferencia de carga de ligando a metal, ( $\pi_{L} \rightarrow \boldsymbol{e}_{g}$ en la figura 5).

Que se verifique una u otra transición, estará determinado por la capacidad donora ó aceptora de los ligandos y la capacidad de oxidarse ó reducirse del metal central.

TCS: por último, la transferencia de carga desde el metal hacia el solvente puede, en ocasiones, también observarse. La posición de la banda de absorción depende de la facilidad que tenga el solvente para reducirse y el centro metálico para oxidarse. Estas transiciones donde la carga se mueve hacia el solvente se denominan transferencia de carga al solvente, y pueden llegar a tener valores de $\varepsilon$ de $10^{3} \mathrm{M}^{-1} \mathrm{~cm}^{-1}$.

$I L$ : Además de las transferencias de carga, pueden presentarse transiciones en donde solamente están involucrados orbitales de los ligandos y se denominan transiciones centradas en el ligando ó transiciones intraligando, $\left(\pi_{L} \rightarrow \pi_{L}{ }^{*}\right.$, en la figura 5). Estas transiciones son relativamente poco afectadas por los centros metálicos (se corren 
levemente hacia el rojo con respecto a la correspondiente transición $\pi \rightarrow \pi^{*}$ para el ligando libre), ocurren típicamente en el ultravioleta $(\lambda<350 \mathrm{~nm})$ y poseen un alto coeficiente de extinción $\left(\varepsilon \geq 10^{4} \mathrm{M}^{-1} \mathrm{~cm}^{-1}\right)$.

CM: otro tipo de transición que puede ocurrir es aquella en la cual están involucrados los orbitales $d$ del metal y se denominan transiciones centradas en el metal, ó transiciones $\mathrm{d} \rightarrow \mathrm{d}, \quad\left(t_{2 g} \rightarrow e_{g}\right.$ en la figura 5). Estas transiciones están formalmente prohibidas en una geometría octaédrica resultando en absorciones muy débiles $\left(\varepsilon \approx 10-100 \mathrm{M}^{-1} \mathrm{~cm}^{-1}\right)$, usualmente en la región visible. Por otro lado estas transiciones involucran la promoción de un electrón hacia un orbital de naturaleza antiligante $\left(\mathrm{d}_{\sigma}{ }^{*}\right.$-metal) el enlace metal-ligando se debilita y por lo tanto, las transiciones $\mathrm{d} \rightarrow \mathrm{d}$ comúnmente resultan en reacciones fotoquímicas de sustitución de ligandos. Los complejos tricarbonílicos de $\operatorname{Re}^{\mathrm{I}}$ prácticamente no experimentan este tipo de transiciones debido al gran efecto de campo ligando que producen los carbonilos. Por lo tanto, los orbitales d se encuentran separados por una gran energía y, como consecuencia de ello, presentan una notable estabilidad en relación con el cambio de ligandos.

De esta manera, las absorciones que se observan, en los carbonilos de $\operatorname{Re}^{\mathrm{I}}$, corresponden, generalmente, a las transiciones fuertemente permitidas TCML e IL.

La transición $\left(\mathrm{t}_{2 \mathrm{~g}}\right)^{6} \rightarrow\left(\mathrm{t}_{2 \mathrm{~g}}\right)^{5}\left(\pi^{*}\right)^{1}$, es la dominante en los complejos estudiados a las energías fotónicas estudiadas en esta tesis $\left(\lambda_{\text {exc }} \sim 350 \mathrm{~nm}\right)$. Dicho estado excitado puede visualizarse como conteniendo un hueco en el orbital $t_{2 g}$, con un electrón sobre el sistema del ligando. En el estado excitado el complejo es una especie química con varios canales de 
reactividad posibles. A continuación se describen los posibles caminos de desactivación de este tipo de complejo en estado excitado.

\section{Fotofísica}

Las características de los estados excitados de complejos del tipo $\mathrm{Re}(\mathrm{CO})_{3} \mathrm{~L}^{+}$pueden ser resumidas y representadas en un diagrama de Jablonski, figura 7.

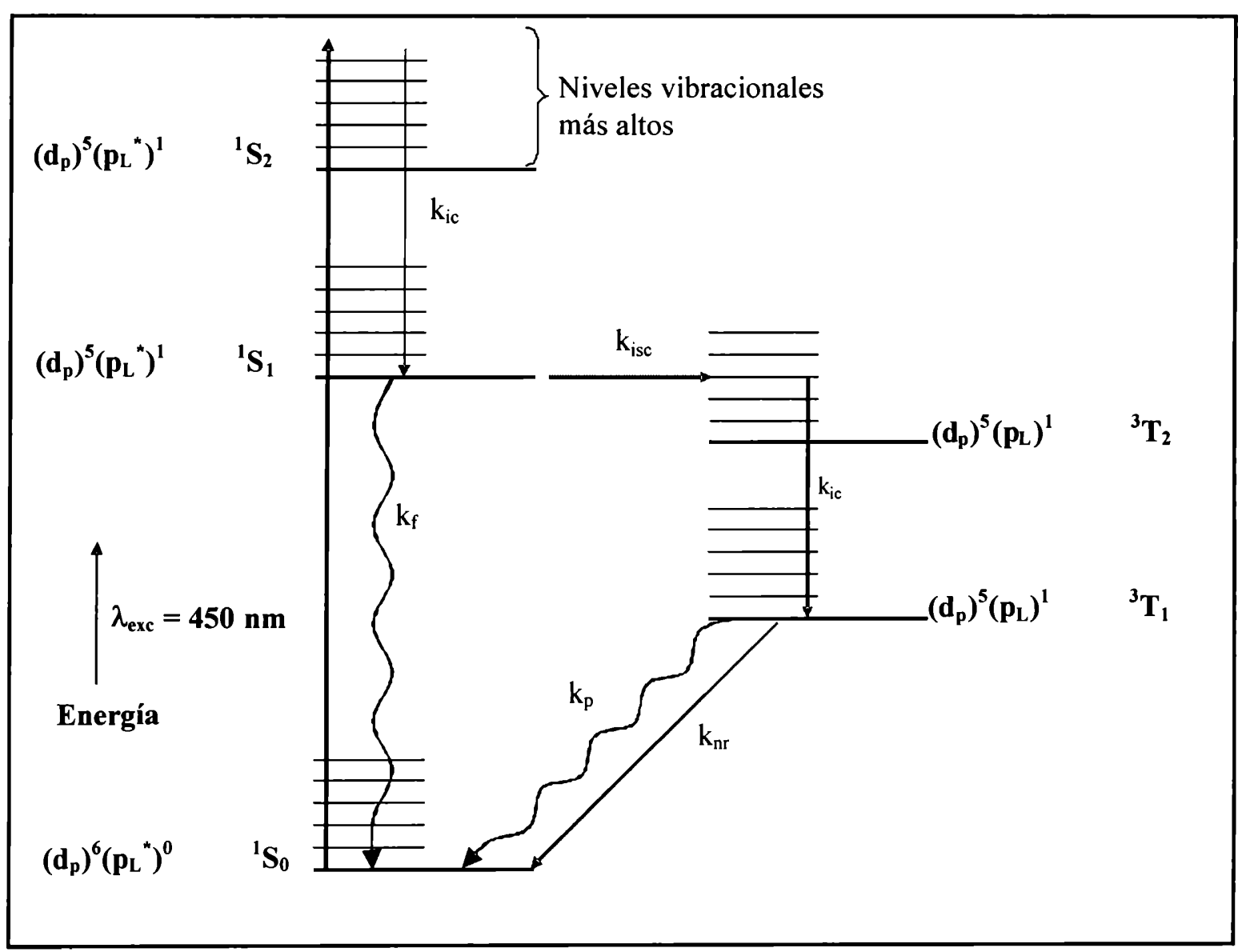

Figura 7: Diagrama de Jablonski que representa los procesos de desactivación de los estados excitados del complejo $\left.\mathrm{Ru}\left(2,2^{\prime} \text { bipi }\right)_{3}{ }^{+2} \cdot\right\}$ caminos radiativos, $\uparrow$ caminos no radiativos. 
En general, se puede considerar que las moléculas del complejo en su estado basal, presentan una distribución electrónica en donde todos sus electrones se encuentran apareados, es decir, es de naturaleza singlete (la multiplicidad global de la molécula es igual a 1). Si la promoción de un electrón a algún estado excitado de energía mayor, luego de la absorción de luz, se produce manteniendo las coordenadas nucleares del estado fundamental, es decir, los cambios electrónicos ocurren mucho más rápidamente que los reacomodamientos nucleares, entonces estas transiciones electrónicas se dice que obedecen al principio de Franck-Condon, y a los estados generados inmediatamente después de la absorción de luz se los denomina estados de Franck-Condon.

Debido a que las transiciones en las cuales se mantiene la misma multiplicidad de spin son completamente permitidas (y por lo tanto las absorciones serán intensas) se asume que los estados excitados poblados también serán de naturaleza singletes. Una transición a un estado triplete (en la cual el electrón promovido sufrió un cambio de spin) es prohibida por spin y por lo tanto existirá una baja probabilidad de poblar ese estado desde uno basal singlete. Por lo tanto las absorciones, si las hubiera, serán muy débiles. Sin embargo, como veremos más adelante, estos estados se pueden poblar siguiendo otros caminos.

Para este tipo de complejos al irradiar con longitud de onda de baja energía (región visible del espectro electromagnético), se pueblan estados singletes de transferencia de carga, ${ }^{I} \boldsymbol{T C M L}$, generalmente el correspondiente al de menor energía $\left(\mathrm{S}_{1}\right)$, aunque otros de niveles más altos se pueden poblar también en forma directa $\left(S_{2}, S_{3}, \ldots\right.$ etc). En estos casos, luego de la absorción, se produce un proceso de relajación rápida, antes que cualquier otro proceso, hacia el estado excitado más bajo ${ }^{1} \boldsymbol{T C M L}$ (ley de Kasha), debido a la reorganización de los enlaces en toda la estructura molecular producto de los cambios en la configuración electrónica. Este paso de disipación de energía en forma no radiativa se la 
denomina conversión interna y ocurre siempre entre estados de igual multiplicidad. La constante de velocidad específica que caracteriza este proceso de denomina $k_{i c}$ y generalmente es del orden de $10^{13} \mathrm{~s}^{-1}$, tanto en sistemas orgánicos como inorgánicos.

El estado excitado ${ }^{l} \boldsymbol{T C M L}$ puede continuar ahora su camino de regreso al estado de energía basal $\left(\mathrm{S}_{0}\right)$ siguiendo pasos de desactivación radiativos y no radiativos. El camino radiativo, es decir, la pérdida de energía por emisión de luz, se conoce como fluorescencia cuando los estados involucrados poseen la misma multiplicidad $\left(\mathrm{S}_{1} \rightarrow \mathrm{S}_{0}\right)$, siendo $k_{f}$ la constante de velocidad. Tanto en sistemas orgánicos como inorgánicos $k_{f}$ toma valores del orden de $10^{8} \mathrm{~s}^{-1}$.

Se denomina cruzamiento intersistemas a una transición no radiativa entre estados de distinta multiplicidad $(\Delta S=1)$ la cual está formalmente prohibida por spin. Esta regla se cumple, en general, en moléculas orgánicas que contienen átomos de bajo número atómico y por lo tanto estos procesos son muy lentos (la constante de velocidad específica, $k_{i s c}$, es del orden 1-1000 x $10^{6} \mathrm{~s}^{-1}$ ). En sistemas inorgánicos, en cambio, las diferencias pueden ser sustanciales. Estas diferencias se deben, en parte, a los altos valores de las constantes de acoplamiento spin-órbita asociadas con los altos pesos atómicos de los iones metálicos. El incremento del acoplamiento spin-orbital causa un efecto de mezcla entre los estados singletes y tripletes lo cual, de alguna manera, atenúa (flexibiliza) la regla de selección anterior. Por este motivo, estados tripletes pueden poblarse eficientemente desde estados singletes cercanos más altos y las $k_{i s c}$ correspondientes serán del orden de $10^{9}-10^{12} \mathrm{~s}^{-1}$. Así, estas transiciones son muy rápidas e indistinguibles de la conversión interna la cual no tiene ninguna prohibición. 
El estado ${ }^{3} \boldsymbol{T C M L}$ de más baja energía, $\mathrm{T}_{1}$, es el estado excitado responsable, en esta familia de complejos, de la luminiscencia y la reactividad química que se monitorean en las medidas de emisión en estado estacionario, emisión resuelta en el tiempo y experimentos de flash fotólisis. En efecto, una vez poblado, $T_{1}$ la desactivación de este estado para retornar al estado basal puede seguir un camino no radiativo por el cual la energía es disipada como calor hacia los alrededores, a través de vibraciones moleculares internas de altas frecuencias. Este proceso posee una constante de velocidad específica denominada $k_{n r}$.

Además, la emisión espontánea de un fotón de luz puede ocurrir desde este estado ${ }^{3} T C M L$, siguiendo un camino radiativo de desactivación con constante $k_{r}$. En sistemas orgánicos, a este paso radiativo en el cual los estados involucrados poseen diferente multiplicidad de spin se lo denomina fosforescencia $\left(\mathrm{T}_{1} \rightarrow \mathrm{S}_{0}\right)$ y la constante de velocidad es $k_{p}$. Este modo de desactivación está prohibido por spin y por lo tanto $k_{p}$ toma valores entre $0,02-100 \mathrm{~s}^{-1}$ permitiendo hacer una clara distinción fenomenológica, entre fluorescencia y fosforescencia, mediante los tiempos de vida de la emisión.

En sistemas inorgánicos esta distinción puede ser incierta debido a la característica de mezcla que poseen los estados excitados y $k_{p}$ puede tomar valores entre $10^{2}-10^{7} \mathrm{~s}^{-1}$. Por esta razón en complejos de metales de transición se utiliza el término luminiscencia para describir el camino radiativo correspondiente. Se puede asumir, como regla para complejos polipiridínicos de metales de transición $\mathrm{d}^{6}$, que el decaimiento no radiativo domina y es más importante que el radiativo. 


\section{i. Decaimiento radiativo}

El análisis del decaimiento radiativo es muy importante para establecer la dinámica de los estados excitados y provee, usualmente, el primer indicio sobre el carácter del/los estado/s excitado/s de más baja energía en esta familia de complejos. La emisión típica del estado excitado $T C M L$ corresponde a una banda ancha y sin estructura a temperatura ambiente en solución. La forma de la banda de emisión, su máximo de energía $E_{\mathrm{em}}$, rendimiento cuántico $\phi_{\mathrm{em}}$, tiempo de vida $\tau_{\mathrm{em}}$, etc., son datos experimentales directamente medibles, como se describirá más adelante. El rendimiento cuántico de emisióm, $\phi_{\mathrm{em}}=k_{r} /$ $\left(k_{r}+k_{n r}\right)$ dependiente de la longitud de onda de excitación, indica un eficiente cruzamiento entre sistemas para este tipo de complejos, es decir, el pasaje de ${ }^{1} \boldsymbol{T C M L} \rightarrow{ }^{3} \boldsymbol{T C M L}$ se produce con eficiencia $\sim 1$. Como se mencionó anteriormente, la presencia de un átomo "pesado" permite una rápida formación del ${ }^{3} \boldsymbol{T C M L}$ desde el ${ }^{l} \boldsymbol{T C M L}$ como consecuencia de la alta constante de acoplamiento spin-órbita, impartiendo algún carácter singkese al estado ${ }^{3}$ TCML. Por esta razón, la denominación de estados singlete y triplete para estos complejos no es estrictamente correcta aunque el formalismo se mantiene. El tiempo de vida del estado excitado emisor está relacionado con la inversa de la suma de las constantes correspondientes a los pasos de decaimiento, $\tau_{\mathrm{em}}=\left(k_{r}+k_{n r}\right)^{-1}$. Como se mencionó anteriormente, la desactivación del estado excitado está dominado por el mecanismo no radiativo, es decir $k_{n r} \gg$. $k_{r}$ y por lo tanto $\tau_{\mathrm{em}} \equiv 1 / k_{n r}$. De esta manera se podría calcular fácilmente $k_{r}$ y $k_{n r}$ una vez determinados experimentalmente el $\phi_{\mathrm{em}} \mathrm{y} \tau_{\mathrm{em}}$. Siguiendo con el decaimiento del estado excitado ${ }^{3} \boldsymbol{T C M L}$ al estado fundamental singlete, nuevamente vemos que este paso está formalmente prohibido por spin, pero el mismo efecto de acoplamiento 
spin-órbita permite un decaimiento luminiscente del estado excitado con tiempos de vida del orden de $0,1-2 \mu$ s para cromóforos como el $\mathrm{Ru}\left(2,2^{\prime} \text { bipi }\right)_{3}{ }^{+2^{*}}$. Estos tiempos son relativamente de vida larga comparado con los de transiciones singlete-singlete puras de sistemas orgánicos cuyo $\tau_{\mathrm{em}} \sim 1 \mathrm{~ns}$ (fluorescencia), pero más cortos que los tiempos en que se efectúa una transición fosforescente, que son del orden del milisegundo, para estos mismos sistemas.

\section{ii. Efectos del solvente}

La emisión originada en un estado excitado de $\boldsymbol{T C M L}$ es también sensible a la polaridad del solvente. El estado excitado genera un gran cambio en el momento dipolar con respecto al estado fundamental, producto de la transferencia de un electrón desde el metal hacia el ligando alojándose ahora sobre esta parte del complejo. Esto provoca una mayor estabilización del estado excitado en solventes más polares. El origen microscópico de esto, está relacionado con el efecto que sobre la constante dieléctrica tiene el solvente y puede ser racionalizado en el siguiente contexto: cuando el estado excitado de más baja energía ${ }^{3} T C M L$ sigue los pasos de relajación, las moléculas del solvente se reorientarán en función del momento dipolar generado por el estado excitado. Los solventes más polares podrían producir una mayor reorientación de sus moléculas generando un efecto de mayor estabilización en el estado excitado. Obviamente, para que esta dinámica tenga lugar, la redistribución del solvente debe ocurrir mucho más rápidamente que el decaimiento del estado excitado. Un rasgo interesante de esta dinámica del solvente es que bajo condiciones de baja temperatura $(120-140 \mathrm{~K}$ en mezclas de etanol -metanol 4:1 (vol/vol), 4:1 
EtOH/MeOH) la solución se encuentra en un estado intermedio vidrio-fluido y los cambios en la reorganización del solvente ocurren en una escala de tiempo similar a la del decaimiento del estado excitado, observándose cinéticas no exponenciales. Al enfriar hasta $77 \mathrm{~K}$ la solución se transforma en una matriz rígida de vidrio y la cinética de tipo exponencial para el decaimiento del estado excitado reaparece. Sobre la energía de la emisión se produce un dramático corrimiento hacia el azul producto del denominado efecto "rigidocrómico". Otro rasgo común de los espectros a baja temperatura es la observación de emisión estructurada.

\section{iii. $\operatorname{Emisión}^{3}\left(\pi^{*} \rightarrow \pi\right)$}

A pesar de la prevalencia de la emisión del estado excitado ${ }^{3} \boldsymbol{T C M L}$ a temperatura ambiente, otro tipo de emisión ha sido observada en complejos de metales de transición $\mathrm{d}^{6}$.

En particular para los complejos tricarbonílicos de $\mathrm{Re}^{\mathrm{I}}$ con ligandos bidentados de tipo diimina, la emisión ${ }^{3}\left(\pi^{*} \rightarrow \pi\right)$ se origina en un estado de tipo $\boldsymbol{I} \boldsymbol{L}$ del complejo; se observa más claramente a $77 \mathrm{~K}$ y puede ser fácilmente asignada por la fuerte similitud con el espectro de fosforescencia del ligando no coordinado. Estos ligandos muestran estructura vibrónica similar a la de los espectros de emisión del TCML a $77 \mathrm{~K}$, sin embargo el máximo de emisión de $T C M L$ está corrido más hacia el rojo $\left(\sim 2500 \mathrm{~cm}^{-1}\right)$ que el del ${ }^{3}\left(\pi^{*}\right.$ $\rightarrow \pi$ ) comparado con la fosforescencia del ligando. La banda de emisión es angosta y estructurada aún a temperatura ambiente aunque muchas veces se encuentra enmascarada por la emisión coexistente de la transferencia de carga. Los estados ${ }^{3} \boldsymbol{I L}$ en el complejo son usualmente de vida larga aunque sus tiempos de vida son más cortos comparados con los del ligando libre. Estos estados muestran una pequeña ó nula dependencia con el solvente debido a que no se produce un gran cambio en el momento dipolar cuando el estado 
excitado IL es generado. La introducción en los complejos de ligandos polipiridínicos con estructuras aromáticas que poseen un sistema $\pi$. fuertemente deslocalizado, aumenta la posibilidad de generar estados excitados de energía más baja centrados en el ligando. Ejemplos de ellos se encuentran en la bibliografía para diferentes complejos de $\operatorname{Re}^{\mathrm{I}}$.

\section{iv. Quenching bimolecular}

Otro método para la desactivación del estado excitado emisor ${ }^{3}$ TCML es por transferencia de electrones ó energía hacia donores ó aceptores apropiados. Cuando se produce la transferencia de carga metal-ligando, el electrón se ubica en un orbital $\pi^{*}$ de alta energía del ligando, y a la vez, se genera un hueco en el orbital de baja energía $\mathrm{d}_{\mathbb{4}}$ del metal. Por lo tanto el potencial de ionización decrece y la afinidad electrónica se incrementa cuando la molécula pasa del estado electrónico fundamental al estado electrónico excitado. Esto quiere decir que un estado electrónico excitado, en general, será un mejor reductor y un mejor oxidante que el estado electrónico fundamental. Por lo tanto, en presencia de aceptores y donores de electrones ocurre una rápida transferencia de electrones que desactiva el estado excitado ${ }^{3}$ TCML. En complejos tricarbonílicos de $\mathrm{Re}^{\mathrm{I}}$ han sido ampliamente utilizadas aminas reductoras como la trietanolamina (TEOA) y trietilamina (TEA). Como ejemplo se muestra en la ecuación siguiente la reacción entre TEOA y un complejo de $\operatorname{Re}^{\mathrm{I}}$ con ligandos 4-fenilpiridina (4-phpy):

$\operatorname{Re}(\mathrm{CO})_{3}\left(4-\right.$ phpy $_{3}{ }^{+*}+\mathrm{TEOA}-\mathrm{k}_{\mathrm{Q}} \operatorname{Re}(\mathrm{CO}) 3(4-\mathrm{phpy})_{3}{ }^{\bullet}+\mathrm{TEOA}^{+}$ 
Posteriormente, la recombinación rápida de los productos regenera el estado fundamental.

El estado excitado ${ }^{3}$ TCML también puede ser empleado en reacciones de sensibilización por transferencia de energía a estados tripletes de moléculas orgánicas como ocurre entre el estado excitado del complejo $\mathrm{Ru}\left(2,2^{\prime} \mathrm{Bpy}\right)_{3}{ }^{+2}$ y el antraceno.

\section{Fotoquimica}

La fotoquímica de estos complejos de metales de transición y en particular los que tienen al $\operatorname{Re}^{\mathrm{I}}$ como metal central, involucra, en la mayoría de los casos, reacciones de transferencia de electrones del estado excitado ${ }^{3} T C M L$ debido, fundamentalmente, como ya se mencionó, a que son fuertes oxidantes y reductores comparado con el estado fundamental. Los estados excitados de estos complejos son usualmente de vida larga (ns$\mu \mathrm{s})$ lo que le permite participar en reacciones rápidas de transferencia de electrones ó de energía similares a las que se describieron anteriormente. A pesar de que los complejos de $\operatorname{Re}^{\mathrm{I}}$ poseen mayor estabilidad fotoquímica que complejos de otros metales, ante la pérdida de ligandos, se han informado algunos ejemplos de este tipo de reacciones, por ejemplo, para el $\operatorname{Re}(\mathrm{CO})_{3}(4-\text { phpy })_{3}{ }^{+}$excitando con pulsos de luz de $355 \mathrm{~nm}$ :

$$
\operatorname{Re}(\mathrm{CO})_{3}(4-\text { phpy })_{3}{ }^{+}+\text {hụ } \longrightarrow \operatorname{Re}(\mathrm{CO})_{3}(4-\text { phpy })_{2}{ }^{+2}+4-\text { phpy }^{\circ-}
$$

Por otro lado la alta reactividad de las especies reducidas puede ser utilizada en fotocatálisis. Por ejemplo, la fotoexcitación del complejo $\mathrm{ClRe}(\mathrm{CO})_{3}\left(2,2^{2}\right.$ bipi) en presencia 
de un quencher reductivo como la TEOA produce el radical $\mathrm{ClRe}(\mathrm{CO})_{3}\left(2,2^{\prime} \mathrm{Bpy}\right)^{\bullet-}$ el cual pierde el ligando $\mathrm{Cl}^{-}$. El radical resultante reacciona posteriormente con $\mathrm{CO}_{2}$ presente en la solución resulta en una reducción fotocatalizada del $\mathrm{CO}_{2}$ para dar $\mathrm{CO}$ vía un complicado mecanismo de reacción.

Similar resultado se obtuvo utilizando como fotocatalizador el complejo $\mathrm{ClRe}(\mathrm{CO})_{3}(4-\mathrm{phpy})_{2}$.

La oxidación del estado excitado, las reacciones de transferencias de electrones intramoleculares ó estudios sobre la dinámica de fragmentación de un ligando por oxidación, son otras de las formas en que estos complejos muestran su variada y versátil reactividad fotoquímica. También fue observado un tipo completamente diferente de fotoreactividad que involucra una ruptura homolítica de un enlace Re-ligando produciendo radicales que podrían encontrar aplicaciones en la fotoiniciación de reacciones de polimerizaciones por radicales.

La fotoquímica y fotofísica de estos complejos dependerá fuertemente de la composición de la esfera de coordinación, es decir, de la naturaleza particular de los ligandos.

Las reacciones correspondientes a estados centrados en el metal ó d-d para esta familia de compuestos son de menor importancia fotoquímica debido a que estos estados se encuentran a una energía mayor. 


\section{Capítulo II}

Espectroscopía de absorción 


\section{Capitulo II}

\section{Espectroscopia de absorción}

Un espectro de absorción es un registro de la intensidad de la luz absorbida por una muestra en función de la longitud de onda de la luz incidente. Las longitudes de onda absorbidas por la molécula en estudio dan cuenta de la energía que necesitan los electrones que la componen para pasar de un nivel de menor a mayor energía. Por lo tanto las bandas que se observan en el espectro dan información sobre las diferencias energéticas entre los estados electrónicos de una molécula.

Un equipo para el registro de un espectro UV-Visible, especctrofotómetro, debe contar con una fuente de luz estable, un sistema de prismas y/o redes de difracción con el fin de variar la longitud de onda que llega a la muestra en forma continua en la región UV-Visible del espectro electromagnético. Y por último debe poseer un detector capaz de responder linealmente con la intensidad de luz transmitida a través de la muestra.

La muestra puede encontrarse en fase gaseosa, líquida o sólida y la celda que la contiene debe ser transparente a la longitud de onda incidente, por lo tanto es necesario trabajar con celdas de cuarzo para registrar la absorción de longitudes de onda menores o iguales a $300 \mathrm{~nm}$. Si la muestra se encuentra en solución debe hacerse una corrección por el solvente, restando a cada absorbancia de la solución la absorbancia del solvente en las mismas condiciones experimentales.

Los espectros de absorción registrados en esta tesis, se obtuvieron sobre soluciones diluidas del compuesto de interés. El equipo utilizado fue un espectrofotómetro de doble haz Cary3 UV-Visible (Varian, Australia). 
El instrumento posee dos fuentes de irradiación, una para luz ultravioleta y otra para luz visible. Utiliza dos redes de difracción (pre-monocromador y monocromador) para obtener un ancho de banda entre 0.2 y $4 \mathrm{~nm}$ de acuerdo con el ancho de rendija seleccionado. Un sistema de espejos y un par de choppers hacen incidir alternativamente los haces de la muestra y la referencia sobre el fototubo. Este equipo permite registrar espectros entre 190 y $900 \mathrm{~nm}$ y está provisto de un software adecuado para registrar y almacenar los espectros.

Además de utilizarse para caracterizar a las sustancias estudiadas, la espectroscopía de absorción ha sido utilizada para el seguimiento de reacciones de síntesis y purificación y para fijar condiciones experimentales como por ejemplo absorción a determinada longitud de onda 


\section{Capítulo III}

Espectroscopía de emisión estacionaria 


\section{Capitulo III}

\section{Espectroscopía de emisión estacionaria}

Un espectro de emisión es la distribución espectral de la emisión de la muestra en estudio, medida excitando con una longitud de onda fija $\left(\lambda_{\text {exc }}\right)$. Inversamente, un espectro de excitación consiste en la dependencia de la intensidad de emisión a una dada longitud de onda $\left(\lambda_{\text {obs }}\right)$, con la variación de la longitud de onda de excitación.

En la figura 1 se muestra un diagrama esquemático del sistema óptico del espectrofluorómetro Perkin-Elmer LS 50B utilizado para los experimentos realizados en este trabajo.

Básicamente, los espectros de emisión en estado estacionario, se obtienen excitando con luz monocromática desde una fuente de excitación luminosa (una lámpara de arco de Xenón en el espectrofluorómetro Perkin-Elmer LS 50B), focalizando el haz (cuya pureza espectral se obtiene del monocromador de excitación), sobre la cubeta. Para evitar complicaciones por efecto de la luz dispersada la emisión de la muestra se detecta a $90^{\circ}$ con respecto al haz de excitación.

El espectro de emisión se obtiene por barrido de un segundo monocromador (monocromador de emisión) situado entre la muestra y el detector, un tubo fotomultiplicador R928 sensible al rojo. 


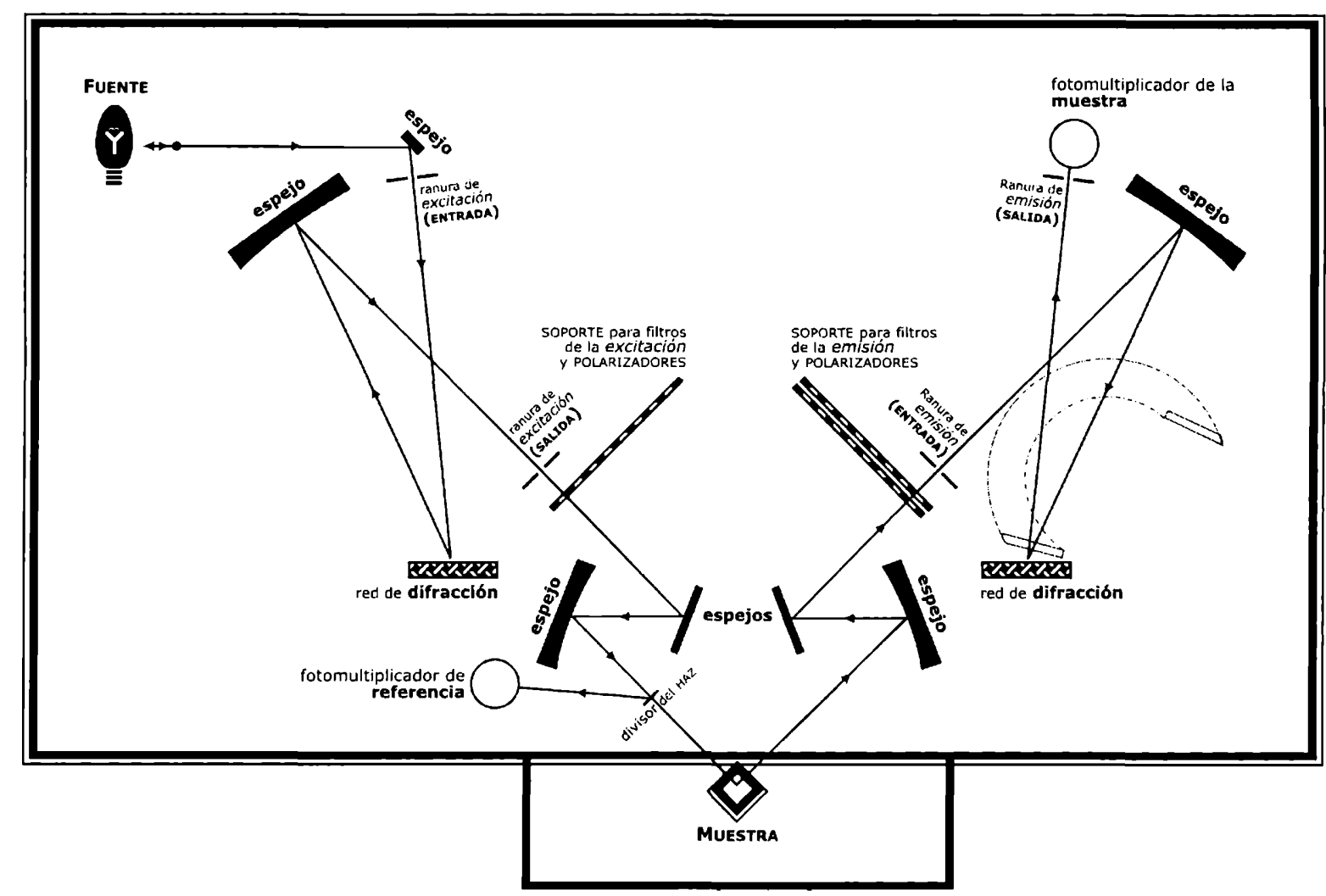

Figura 1: Diagrama esquemático del sistema óptico del espectrofluorómetro utilizado para los experimentos de emisión en estado estacionario

La lámpara de Xenón emite con alta intensidad en todo el rango de longitudes de onda por encima de $250 \mathrm{~nm}$ por lo tanto es muy útil para trabajar en la región UV-Visible. Por esta razón se utilizaron celdas de cuarzo de calidad óptica similar a la utilizada en las medidas de láser flash fotólisis. Las soluciones a medir fueron previamente deaereadas con $\mathrm{N}_{2}$ ultrapuro. El espectro de emisión obtenido es corregido haciendo la diferencia entre éste y el espectro de emisión del solvente ó matriz tomado en las mismas condiciones, y al espectro así obtenido se lo corrige por la respuesta del instrumento (la sensibilidad del detector y la eficiencia de los monocromadores varían con la longitud de onda). Esta 
corrección se realiza dividiendo la señal de emisión de la muestra por la respuesta del instrumento, que en nuestro caso se obtuvo dividiendo el espectro corregido de una sustancia de referencia por el espectro de la misma sustancia tomado en nuestro equipo. El denominado espectro de emisión corregido hace referencia, generalmente, a este tipo de corrección.

Para obtener un espectro de excitación se realiza un barrido con el monocromador de excitación manteniendo fijo el monocromador de emisión, generalmente, a la longitud de onda del máximo de emisión. Los espectros así obtenidos sufren distorsión debido, principalmente, a la dependencia de la intensidad de la luz de excitación con la longitud de onda, es decir, la lámpara no provee una misma intensidad de luz en todo su espectro de emisión. Por lo tanto se debe realizar una corrección de la señal de intensidad de la emisión de la muestra transformándola en una señal proporcional al número de fotones incidentes.

En nuestro equipo y mediante el fotomultiplicador de referencia el sistema calibra y corrige automáticamente, de manera que los espectros de excitación corregidos se obtienen en forma directa.

La dependencia con la longitud de onda de la respuesta del monocromador de emisión y del detector que recibe la emisión de la muestra no son importantes debido a que la longitud de onda de emisión se mantiene constante durante el experimento.

Otro factor a tener en cuenta en todos los experimentos de luminiscencia es la posibilidad de que la intensidad aparente de la emisión y su distribución espectral sean dependientes de la densidad óptica de la muestra y de la geometría del sistema. Para la geometría más común usada en espectrofluorometría, que implica la observación de la emisión en ángulo recto de una cubeta iluminada centralmente, se puede demostrar que si la absorbancia de la muestra a la longitud de onda de excitación es de 0,1 la intensidad en el 
centro de la cubeta, I, es 0,88 .Io, donde Io es la intensidad incidente sobre la cubeta. Como la intensidad de la luminiscencia observada es proporcional a la intensidad de la luz de excitación, el rendimiento cuántico aparente será alrededor de un $10 \%$ menor que el observado con una solución infinitamente diluida. Este es el denominado efecto de filtro interno. Tal fenómeno puede disminuir la intensidad de excitación en el punto de observación, ó disminuir la emisión observada por absorción de ésta. Por lo general, y para minimizar este efecto, los espectros de emisión y excitación se tomaron utilizando soluciones con una absorbancia a la longitud de onda de excitación, $\mathrm{A}_{\lambda \operatorname{exc}} \leqslant 0,1$.

Como se mencionó anteriormente, otro parámetro directamente medible en experimentos de emisión en estado estacionario y de vital importancia para la caracterización del estado excitado emisor de un complejo dado, es el rendimiento cuántico de emisión. Los rendimientos cuánticos dan cuenta de la eficiencia de un proceso en términos de la cantidad de energía lumínica absorbida. El rendimiento cuántico de un proceso fotofísico como la emisión de luz, $\phi_{\mathrm{em}}$, se puede expresar como el número de moléculas emisoras respecto de los fotones de luz absorbidos:

$$
\phi_{\mathrm{em}}=\frac{\text { Nro. de moléculas emisoras por unidad de tiempo y por unidad de volúmen }}{\text { Nro. de cuantos absorbidos por unidad de tiempo y por unidad de volúmen }}
$$

Como el número de fotones absorbidos es proporcional a la intensidad de la radiación absorbida, $I_{A}$, y el número de fotones emitidos es proporcional a la intensidad de la radiación emitida, $I_{e m}$, se puede considerar y escribir que:

$$
\phi_{\mathrm{em}}=\frac{I_{c m i}}{I_{A}}
$$


Nuevamente debemos hacer la siguiente consideración: la expresión anterior es estrictamente válida cuando el estado excitado emisor es poblado en forma directa por la absorción de luz, es decir, cuando el rendimiento cuántico coincide con la eficiencia $\eta$. Si, por otro lado, el estado excitado emisor es poblado en forma indirecta siguiendo algún camino no radiativo, desde otro estado excitado que absorbió la luz, entonces el $\phi_{\mathrm{em}}$ se obtiene multiplicando la expresión por la eficiencia del camino seguido. Como se mencionó anteriormente, éste es el caso para los estados excitados ${ }^{3} \mathrm{TCML}$ de los complejos en estudio que son poblados desde el correspondiente singlete a través de un paso de cruzamiento intersistemas. Por lo tanto, la expresión adecuada será:

$$
\phi_{\mathrm{em}}=\eta_{\text {isc }} \frac{\mathrm{Iem}}{\mathrm{IA}_{\mathrm{A}}}
$$

pero como $\eta_{\text {isc }} \sim 1$ la ecuación anterior se vuelve a transformar en la expresión original.

Experimentalmente, los rendimientos cuánticos de emisión de la solución muestra en estudio, se determinan por comparación de la intensidad de la emisión, $I_{e m}$, tomada como el área bajo la curva del espectro de emisión, $I_{\text {muestra }}$ y la intensidad de emisión, tomado en iguales condiciones, de una solución de una referencia de rendimiento cuántico conocido, $I_{\text {referencia. }}$ La referencia a utilizar debería ser ópticamente comparable con la muestra (es decir, tener la misma densidad óptica y camino óptico y similar perfil de energía de emisión). Si la absorbancia de ambas soluciones a la longitud de onda de excitación, $A_{\text {muestra }}$ y $A_{\text {referencia }}$, es diferente, debe realizarse una corrección teniendo en cuenta esta condición. Finalmente, y tomando ambos espectros con el mismo equipo y en idénticas condiciones de temperatura, celda utilizada, abertura de las rendijas, etc., los rendimiento cuánticos de emisión relativo fueron calculados de acuerdo a la siguiente ecuación: 


$$
\phi_{\mathrm{em}}=\left(\frac{\text { Areferencia }}{\text { Amuestra }}\right)\left(\frac{\mathrm{Imuestra}}{\mathrm{I}_{\text {referencia }}}\right) \phi \mathrm{em}, \text { referencia }\left(\frac{\mathrm{n}_{\text {muestra }}}{n_{\text {referencia }}}\right)^{2}
$$

donde $n$ es el índice de refracción del solvente puro empleado en cada una de las soluciones.

Estas medidas requieren, como se mencionó, que las soluciones sean óptimamente diluidas $\left(\mathrm{A} \lambda_{\mathrm{exc}} \leq 0,1\right)$ permitiendo, además, despreciar las diferencias entre los $n$ del solvente puro y la solución. 


\section{Capítulo IV}

Láser flash fotólisis 


\section{Capítulo IV}

\section{Método de flash fotólisis}

\section{Introducción}

El método de flash fotólisis fue desarrollado por R. G. W. Morrish y G. Porter en 1948. Es un método utilizado para la iniciación y el estudio de procesos fotoquímicos. En esta técnica se crea una situación de no-equilibrio en la mezcla de reacción en un intervalo de tiempo corto, generando intermediarios inestables en concentraciones más altas que las que pueden ser obtenidas por otros métodos.

El procedimiento consiste en someter al material en estudio a un corto e intenso flash de luz con lo que se forma una concentración relativamente alta de moléculas excitadas y/ó radicales libres. Las especies transitorias producidas son detectadas por espectroscopía de absorción ó de emisión, pudiéndose seguir la evolución temporal de las mismas.

Al producirse la absorción de luz por una molécula se originan especies en estados electrónicos excitados. Dichas especies pueden sufrir una transformación química permanente es decir, una reacción fotoquímica, o volver al estado fundamental del cual partieron a través de decaimiento radiativo y/ó no radiativo. Estos procesos de decaimiento cubren un amplio intervalo de tiempos desde los picosegundos (para la relajación vibracional en solución), nanosegundos (para los procesos radiativos entre estados de igual multiplicidad), microsegundos (para los procesos radiativos entre estados de distinta 
multiplicidad) y en algunos casos hasta segundos (para algunos radicales y especies químicas de vida larga).

Los estudios fotoquímicos resultan de gran importancia ya que han permitido investigar numerosas reacciones químicas, sus mecanismos y la naturaleza de los intermediarios involucrados. Además su aplicación al estudio de reacciones rápidas se basa en el hecho de que la iniciación fotoquímica es muy conveniente para producir una reacción química en forma homogénea, en un corto intervalo de tiempo.

La resolución temporal está limitada por la duración del flash, que es usualmente de unos pocos microsegundos en el caso de lámparas de plasma (flash fotólisis convencional). También, la intensidad del flash debe ser suficientemente alta como para producir un cambio medible en la composición química, pero de muy corta duración comparada con la vida media de los procesos que quieren estudiarse. Estas, son dos de las limitaciones más importantes de esta técnica, cuando se desea estudiar intermediarios o estados excitados de vida muy corta, usando lámparas pulsadas como fuente de excitación.

\section{Láser flash fotólisis}

El desarrollo y aplicación de fuentes de luz láser, a fines de la década del '60, dio inicio a la técnica de láser flash fotólisis. El descubrimiento de los láseres pulsados proveyó una nueva fuente para la excitación, posibilitando la extensión del método de flash fotólisis desde los microsegundos a las regiones de los nanosegundos, picosegundos y hasta femtosegundos.

En todos los láseres, la absorción de energia proveniente de una fuente externa eleva a un número suficiente de moléculas desde su estado fundamental a un estado excitado de 
modo de permitir que ocurra una inversión de la población a ciertos niveles de energía favorecidos. La emisión espontánea de un fotón de alguna de las moléculas excitadas estimula la emisión de otras emitiendo en fase y cuando la cascada de energía aumenta muy rápidamente, ocurre la acción láser (amplificación de luz por emisión de radiación estimulada, LASER en inglés), produciéndose un haz de radiación intenso y coherente, de ancho de banda pequeño. De esta manera, las características de la luz de excitación láser y que son una ventaja con respecto a las lámparas de plasma, son las siguientes:

1. las potencias de pico pueden ser enormes (109 a 1012 watts)

2. la luz emitida es coherente (los fotones están en fase), monocromática (porque los fotones estimulan fotones de la misma frecuencia), y puede ser enfocada en áreas muy pequeñas

3. el pulso de luz es generalmente simétrico con el tiempo y no está acompañado por una larga cola

4. la duración del pulso luminoso es muy corto (nano al femtosegundo)

Existen una gran cantidad de tipos de láseres que emiten a longitudes de onda característica abarcando amplias regiones del espectro electromagnético, desde los $190 \mathrm{~nm}$ hasta la décima de $\mu \mathrm{m}$. También, la energía y duración del pulso de luz difiere en cada caso, así como la naturaleza de la especie emisora. En función de estos parámetros, los láseres, que pueden ser continuos ó pulsados, se clasifican en varios grupos.

Los láseres de gas continuos de modo acoplado, como los de helio-neón, heliocadmio, argón, y kriptón, producen pulsos menores que el nanosegundo, repetitivos, de relativamente baja energía, que son apropiados para los estudios de emisión. Los láseres de gas pulsados como los de nitrógeno, hidrógeno y monóxido de carbono emiten pulsos de 
luz ultravioleta de hasta unos cientos de kilowatts de potencia, con anchos de pulso de unos pocos nanosegundos.

Láseres pulsados de dióxido de carbono de alta potencia emiten en la región del infrarrojo, cerca de 10,6 $\mu \mathrm{m}$. Los láseres de semiconductores, que pueden ser continuos o pulsados, emiten generalmente bajas potencias en el infrarrojo. Los láseres de líquidos, que incluyen los de colorantes, pueden producir pulsos cuya energía se encuentra entre el ultravioleta cercano y el infrarrojo. Los láseres de estado sólido (rubí, neodimio) pueden producir pulsos de luz de muy alta potencia, y son los que han encontrado el uso más extensivo en láser flash fotólisis. Para excitar en la región UV, son extremadamente importantes y utilizados los denominados láseres de excímeros, ya que emiten con pulsos de luz de muy alta energía ( 35 a $200 \mathrm{~mJ} /$ pulso). Están compuestos por una mezcla de un gas halógeno con un gas noble y la energía, ancho de pulso y frecuencia de emisión dependen de la composición de la mezcla gaseosa.

\section{Componentes del equipo de láser flash fotólisis}

En general, un equipo de láser flash fotólisis debe incluir los componentes mostrados en la figura 1:

1. una celda que contiene la muestra, que puede ser de geometría y material variable dependiendo del tipo de láser utilizado y del volumen de muestra utilizado.

2. una fuente de luz de análisis, que típicamente puede ser una lámpara de arco de Xe con potencia de la luz emitida dentro del rango de 150 y 1000 watts ó de tungstenohalógeno. 
La luz de análisis es convenientemente colimada y enfocada sobre la celda de reacción, entrando usualmente por una pequeña ventana u orificio (1 ó $2 \mathrm{~mm}$ ). La intensidad de la luz de análisis, para el registro en el nanosegundo, debe ser alta de modo de producir una señal fotométrica con adecuada relación señal / ruido y reducir los efectos de la fluorescencia dispersada.

3. una fuente de excitación láser cuya elección deberá hacerse teniendo en cuenta, fundamentalmente, la energía, longitud de onda de excitación y duración del pulso. El valor de la energía requerida dependerá de los coeficientes de extinción de los estados excitados y/ó intermediarios de reacción bajo estudio y de sus rendimientos cuánticos de formación.

Un sistema versátil es aquel que permite trabajar en un rango entre 1 y $100 \mathrm{~mJ} /$ pulso. La duración del pulso debe ser cuidadosamente seleccionada. Un ancho de pulso entre 5 y 20 ns es común y usualmente aceptado. El $\lambda_{\text {exc }}$ dependerá, fundamentalmente, de la naturaleza del láser en sí mismo (como se describió anteriormente). El haz del láser se concentra sobre la celda con geometría de $90^{\circ}$ cubriendo totalmente el camino óptico del haz de análisis y la imagen de salida de la celda se proyecta sobre la rendija de un monocromador.

4. un sistema de lentes y filtros adecuado para colimar, dirigir y enfocar el haz de luz de análisis y el pulso de la luz de excitación. Los filtros comúnmente usados son de paso de banda, de vidrio de calidad óptica ó soluciones de sustancias que absorben la luz del láser atenuando su energía. Si bien se pueden emplear otros arreglos geométricos la excitación a $90^{\circ}$ es fácil de alinear y asegura bajos niveles de dispersión.

5. un detector de la señal luminosa que consiste típicamente de una combinación de un monocromador y un tubo fotomultiplicador. El monocromador es frecuentemente de 
tipo holográfico y cubre el rango de 230 a $800 \mathrm{~nm}$. El fotomultiplicador toma la señal a la salida del monocromador y genera una señal eléctrica que se amplifica y, controlando el voltaje aplicado, se ajusta a la región de respuesta lineal.

6. un osciloscopio digitalizador que recibe la señal del fotomultiplicador y la convierte a la forma digital y transfiere estos datos a una computadora para el procesamiento posterior (análisis y almacenamiento de los datos).

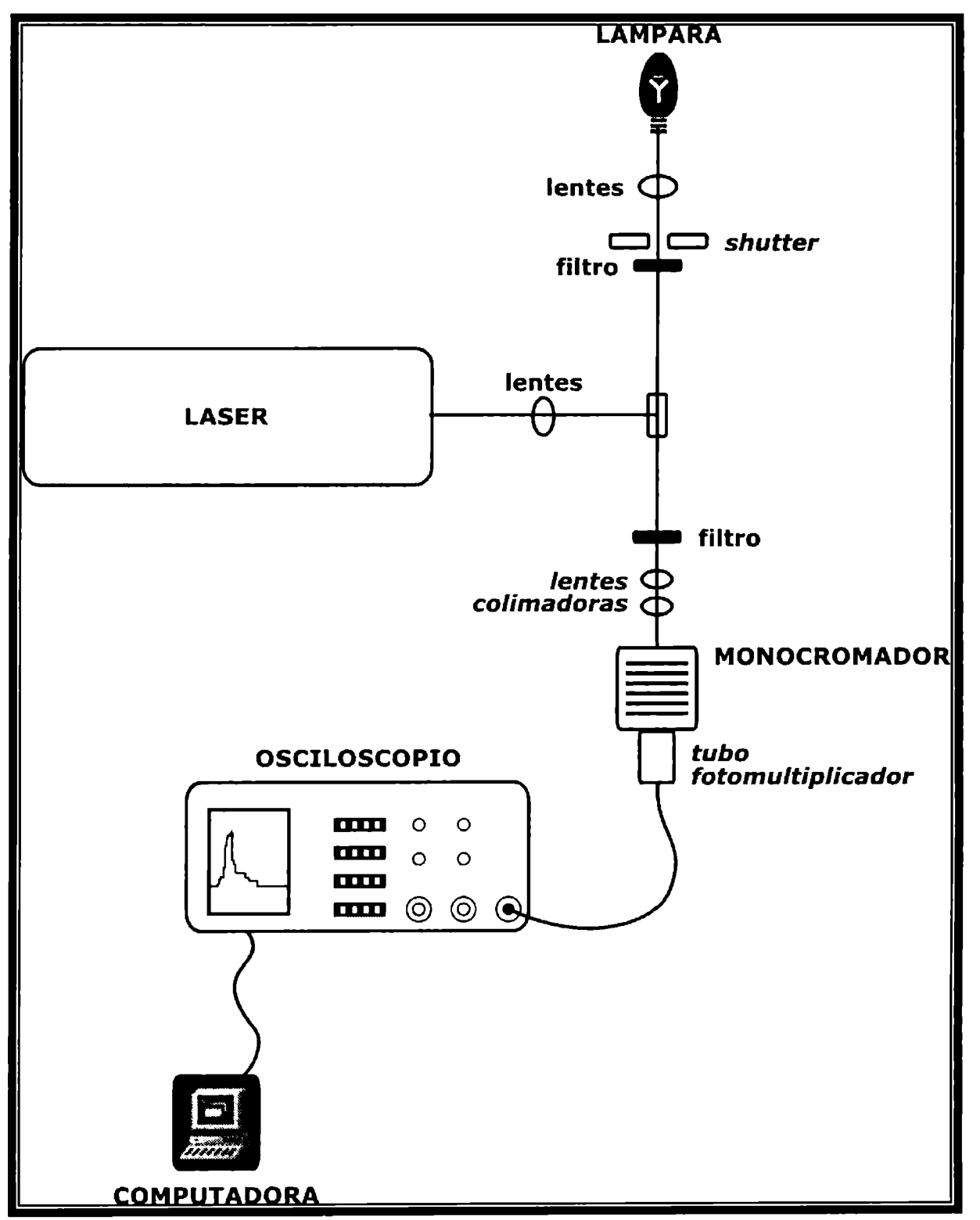

Figura 1: Arreglo experimental y componentes de un equipo de láser flash fotólisis 
Para los experimentos de láser flash fotólisis realizados en este trabajo de tesis se utilizaron

i- un láser de excímero de XeF de $\lambda_{\text {exc }}=351 \mathrm{~nm}$ (energía: $200 \mathrm{~mJ} /$ pulso) Lambda Physic SLL-200, para el registro de la absorbancia de transientes en una escala entre los nanosegundos y los microsegundos.

Fue necesario atenuar la energía del láser a $20 \mathrm{~mJ} /$ pulso, utilizando soluciones de $\mathrm{Ni}\left(\mathrm{ClO}_{4}\right)_{2}$ con la transmitancia óptica apropiada.

ii- Para la observación del espectro de absorción y cinética de decaimiento en la escala entre los femtosegundos y 1.6 nanosegundos, se utilizó un sistema láser Tízafiro CPA-2010 1kHz amplificado Clark MXR y un software para sistemas ultra-rápidos. El equipo provee pulsos de $\lambda_{\mathrm{exc}}=775,387$ ó $258 \mathrm{~nm}$; con un ancho de pulso de 150 fs. Los datos pueden ser registrados en intervalos iguales o mayores a 10 fs. Se utilizó una cubeta de $2 \mathrm{~mm}$ de camino óptico y un flujo lento pero constante de solución con la muestra a investigar.

iii- Para el monitoreo de la emisión resuelta en el tiempo, se utilizó un láser de nitrógeno de $\lambda_{\text {exc }}=337 \mathrm{~nm}$, modelo PTI. La energía del láser es $2 \mathrm{~mJ} /$ pulso y el ancho de pulso a la mitad de la banda es de 200 ps.

iv- Para el seguimiento de la desactivación de la emisión del sistema $\operatorname{PyRe}(\mathrm{CO})_{3}$ Bpy $\quad$ y $\left\{(\mathrm{vpy})_{2} \mathrm{vpyRe}(\mathrm{CO})_{3}(\mathrm{Bpy})^{+}\right\}_{\mathrm{m} \sim 200}$ por diferentes aminas se utilizó un láser Nd-YAG que provee una longitud de onda de excitación de $\lambda_{\mathrm{exc}}=355 \mathrm{~nm}, \mathrm{y}$ un láser de $\mathrm{N}_{2} ; \lambda_{\text {exc }}=337 \mathrm{~nm}$

Todas las soluciones utilizadas para experimentos de láser flash fotólisis fueron previamente deaereadas con $\mathrm{N}_{2}$ ultra puro. 


\section{Procesamiento de los datos}

La señal registrada en el osciloscopio de un sistema de láser flash fotólisis consiste en una representación en función del tiempo de la intensidad de la luz de análisis constituida por algunos cientos de puntos conteniendo la señal de voltaje del fotomultiplicador. Si durante el proceso estudiado se forma una especie transitoria que absorbe más luz que la solución de partida a la longitud de onda de análisis, el osciloscopio registrará una señal del tipo de la que se muestra en la figura 4. En el caso contrario, es decir, cuando la absorción de la especie transitoria es menor que la del material de partida, se pueden registrar señales que corresponden a absorciones negativas ("bleaching"). Es posible también obtener señales en las cuales la absorbancia final sea mayor a la absorbancia antes del pulso del láser, es decir, que no coincida con la línea de base debido a la absorción de fotoproductos ó especies transitorias de vida más larga. En este punto se debe enfatizar que las medidas no corresponden a valores absolutos de la densidad óptica sino a los cambios de la absorción donde el valor cero corresponde a la absorción del material de partida.

Para transformar y obtener entonces la señal correspondiente a la diferencia en la densidad óptica del sistema, $\Delta \mathrm{DO}$, se procede como se describe a continuación. Debe aclararse que, como la especie transitoria absorbe sólo una pequeña fracción de luz, es necesario amplificar la ganancia del osciloscopio, en cuyo caso la posición correspondiente al $0 \%$ de transmitancia no aparecerá en la pantalla del mismo. La respuesta del osciloscopio, $\mathrm{x}$, es proporcional a la intensidad luminosa, I; por lo tanto, pueden plantearse las siguientes relaciones: 


$$
\mathrm{I}_{0}=\mathrm{k} \cdot \mathrm{x}_{0} \quad \mathrm{I}=\mathrm{k} \cdot \mathrm{x}
$$

donde $\mathrm{k}$ es una constante que depende de la sensibilidad del detector. Combinando estas ecuaciones en la ecuación de la ley de Lambert-Beer puede escribirse la siguiente relación:

$$
A=\log \left(\frac{I_{0}}{I}\right)=\log \left(\frac{x_{0}}{x}\right)=\varepsilon_{\lambda} C_{t} I
$$

donde $\varepsilon_{\lambda}$ es el coeficiente de extinción de la especie transitoria a la longitud de onda $\lambda, \mathrm{Ct}$ es su concentración al tiempo t después del pulso del láser y $l$ es el camino óptico.

En la figura 2 se observa que la amplitud de la señal $\mathrm{x}$ está dada en unidades de voltaje. 


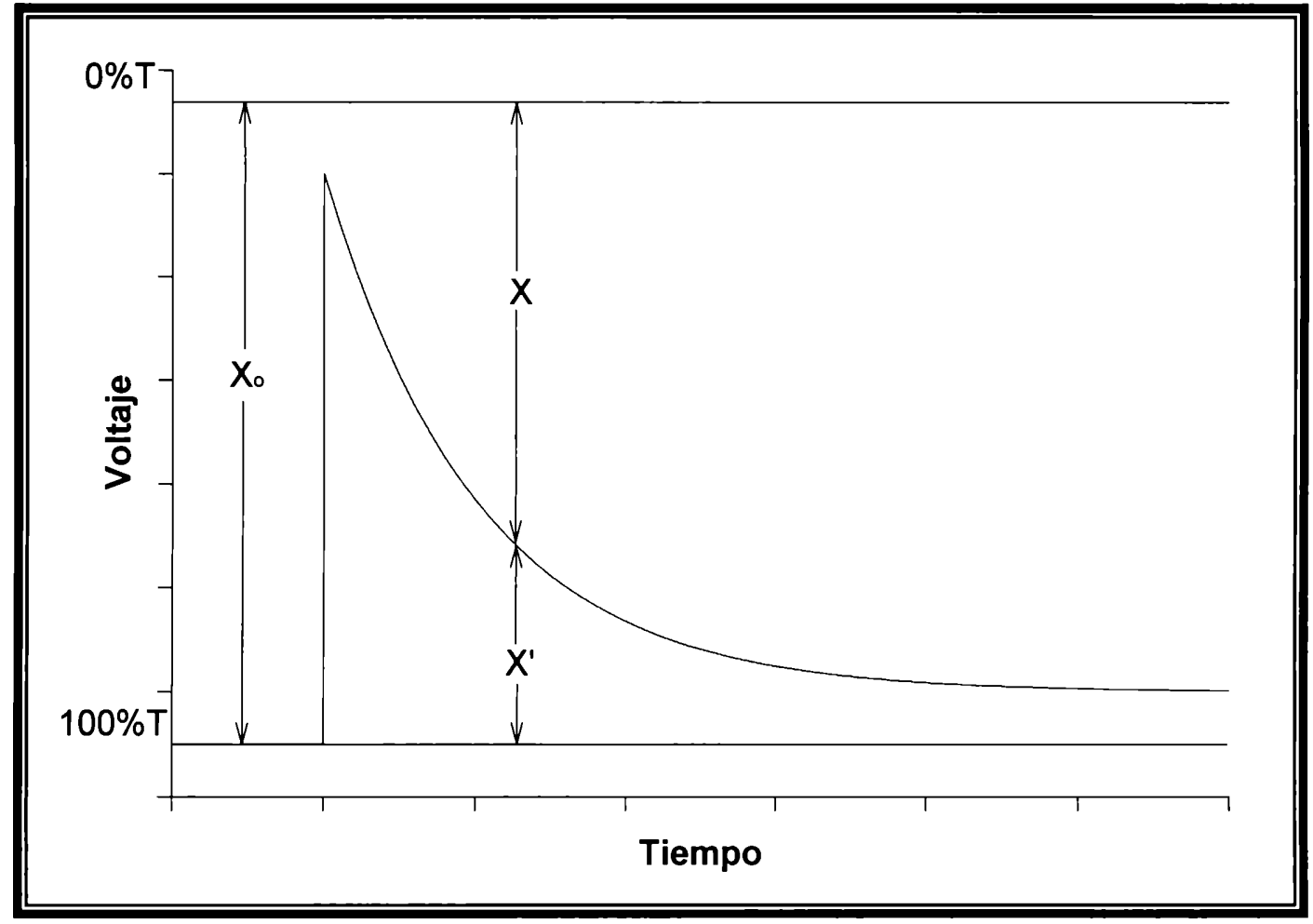

Figura 2: Esquema de una traza obtenida en un experimento de láser flash

fotólisis en la que se observa la desaparición (decaimiento) de una especie transitoria formada luego del pulso del láser.

Con el fin de convertir estos valores en absorbancia o transmitancia porcentual, se ajusta a 1 voltio el $100 \%$ de transmitancia de la luz de monitoreo utilizando un comparador diferencial conectado al osciloscopio. Como la línea correspondiente a $0 \%$ de transmitancia no se registra, no se obtienen las amplitudes x para cada intervalo de tiempo, pero sí las que fueron marcadas como $\mathrm{x}^{\prime}$ en el esquema de la figura 4. La relación entre $\mathrm{x}$ y $\mathrm{x}^{\prime}$ viene dada por:

$$
x^{\prime}=x_{0}-x
$$

Puede plantearse entonces, teniendo en cuenta que $\mathrm{x}_{0}=1000 \mathrm{mV}$, la siguiente ecuación:

$$
A=\log \left(\frac{I_{0}}{I}\right)=\log \left(\frac{x_{0}}{x_{0}-x^{\prime}}\right)
$$


y por lo tanto:

$$
A=\log \left(\frac{1000}{1000-x^{\prime}}\right)=\varepsilon_{\lambda} C_{t} 1
$$

Usualmente, el sistema, en el equipo utilizado, trabaja asignando en forma automática el valor de xo correspondiente al voltaje previo al pulso del láser realizando la transformación correspondiente. 
Capítulo V

Radiólisis de pulso 


\section{Capítulo V}

\section{Radiólisis de pulso}

\section{Introducción}

La técnica de radiólisis de pulso es una poderosa herramienta para la investigación de especies transientes como iones, radicales y estados excitados, tanto en fase sólida como fase solución.

El principio fundamental de la técnica es la inyección de un corto pulso de radiación ionizante o una partícula cargada sobre una muestra, para producir iones y radicales los cuales son directa o indirectamente observados por varios métodos antes de su desaparición. Todos estos fenómenos pueden ser medidos como una función del tiempo. Por lo tanto pueden ser analizados por este método, la dinámica de átomos, moléculas y sus agregados en sus estados excitados incluyendo excitación, transferencia de energía, transferencia de carga y procesos de decaimiento.

El equipo debe contar básicamente con una fuente de radiación ionizante, una celda de geometría adecuada y un sistema de detección.

Existen varios tipos de fuentes de radiación la más común es la fuente de electrones de alta energía. El acelerador lineal de electrones (LINAC), Van de Graff y Febreton son las tres fuentes de radiación de electrones de alta energía frecuentemente utilizadas.

La duración del pulso es un parámetro esencial para la resolución en el tiempo en experimentos de radiólisis de pulso. Son posibles pulsos de micro, nano y picosegundos. 


\section{Fuentes de radiación de electrones}

Las principales fuentes para generar pulsos de electrones de alta energía son, Febretons, Van de Graaffs y acelerador de microondas (banda -L ó -S).

Los dos primeros tipos de fuentes generan pulsos cortos de corriente continua. En contraste los aceleradores de microondas o LINACS, emplean corrientes de corta duración en forma de un tren de pulsos con un ancho entre 20-30 ps con un espaciado de varios cientos de picosegundos, el efecto es equivalente a un pulso de corriente continua.

Cada una de estas fuentes presenta sus ventajas y limitaciones. Febretons provee altas dosis por pulso, lo cual permite la obtención de una alta concentración primaria de radicales, sin embargo la energía es baja y la velocidad de repetición es pequeña. Este hecho limita el tamaño de muestras líquidas y la uniformidad de la irradiación.

Van de Graaff y Linacs exciben altas velocidades de repetición y moderada energía, pero las dosis por pulso son mucho mas bajas que Febretons.

\section{Técnicas de detección}

Los métodos de detección utilizados en técnicas de radiólisis de pulso son: Espectroscopía de emisión y absorción (UV cercana e IR cercana), Conductividad, epr y nmr resuelta en el tiempo, Absorción de microondas, luz scattering, Espectroscopía Raman resuelta en el tiempo.

Para el estudio de sistemas inorgánicos las técnicas mas utilizadas son espectroscopía de absorción y emisión y conductividad. 


\section{Radiólisis del $\mathrm{H}_{2} \mathrm{O}$}

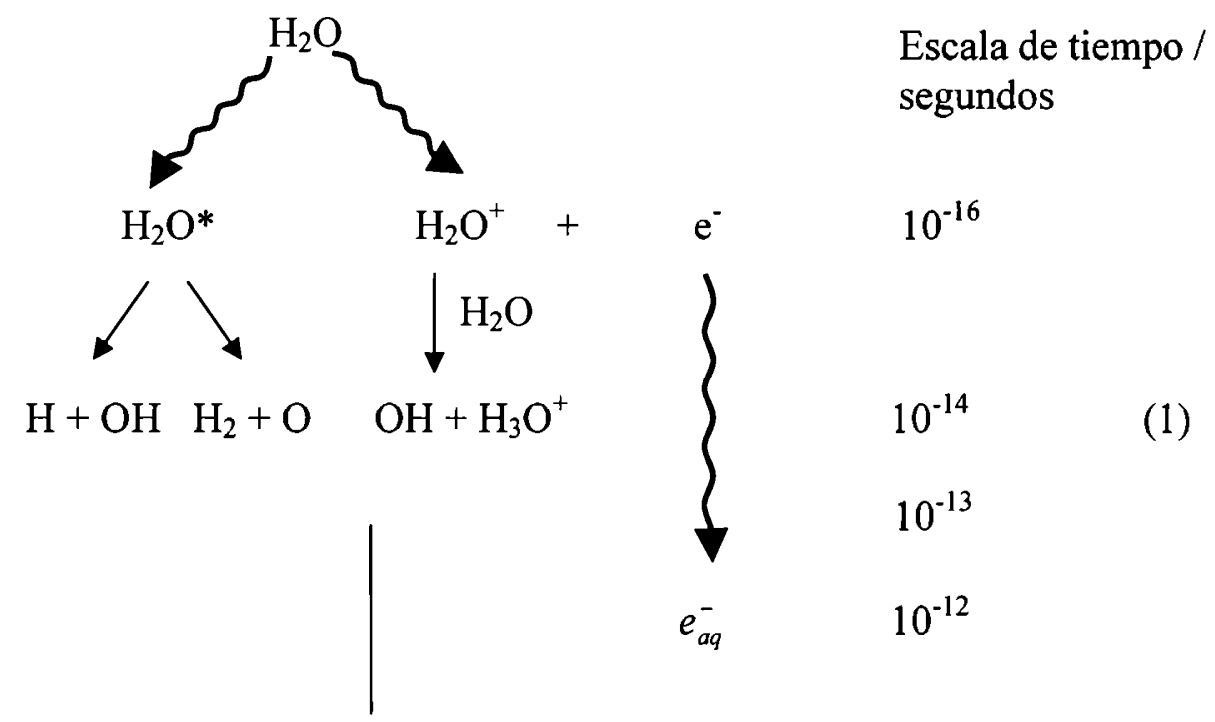

Formación de productos moleculares

y difusión de radicales

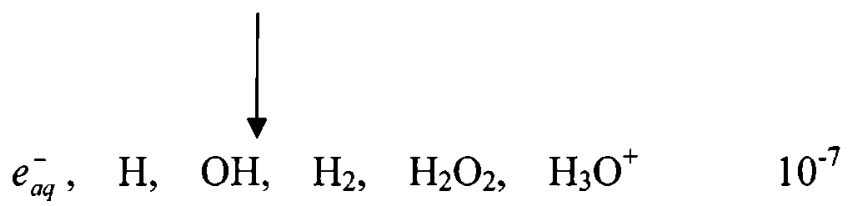

Cantidad Relativa: $\quad 5 \quad 1 \quad 5 \quad \angle 1 \quad \angle 1 \quad 5$

La premisa básica de la radiólisis es que sólo la energía absorbida por el medio puede llevar a un cambio químico. La interacción es por colisión y alcanza a ionizar o a excitar electrónicamente en cuanto la energía del rayo de electrones excede la energía de unión electrónica.

Lo que ocurre generalmente en la práctica, en soluciones acuosas diluidas es que la interacción y el cambio químico se produce en el solvente más que en el soluto. 
Hay dos fenómenos de radiación asociados con la degradación de los electrones, la formación de rayos-x (Radiación Bremsstrahlung) vía desaceleración de un electrón en el campo de los núcleos y radiación Cerenkov, la cual es emitida cuando la velocidad del electrón excede la del medio o matriz.

El rendimiento relativo de especies acuosas primarias es expresado por medio del G-valor, definido como la razón del número de cambio para una especie particular por unidad de energía absorbida. Las dimensiones en S.I, son mol $\mathrm{J}^{-1}$, aunque es común expresarlo en número de moléculas por $100 \mathrm{eV}$. El procedimiento para la determinación del G-valor está descripto en la literatura y es análogo al procedimiento para la determinación del rendimiento cuántico de emisión. Generalmente se utiliza un pulso radiolítico, para la reacción de $\mathrm{OH}$ con $\mathrm{Fe}(\mathrm{CN})_{6}{ }^{4-}$ y $\mathrm{SCN}^{-}$para dar como productos $\mathrm{Fe}(\mathrm{CN})_{6}{ }^{-3}$ (especie estable) y $(\mathrm{SCN})^{\bullet-}$ (especie transitoria).

\begin{tabular}{|c|c|}
\hline Radical & G-Valor \\
\hline$e_{s o l v}^{-}$ & 2.7 \\
\hline $\mathrm{H}$ & 0.55 \\
\hline $\mathrm{OH}$ & 2.8 \\
\hline
\end{tabular}

Tabla 1: G-Valor para los radicales primarios generados por radiólisis del $\mathrm{H}_{2} \mathrm{O}$

\section{Radiólisis de soluciones preparadas en metanol y en mezclas metanol/agua}

Los experimentos de radiólisis de pulso realizados en esta tesis se llevaron a cabo sobre soluciones preparadas en, $\mathrm{CH}_{3} \mathrm{OH}$, deaereadas con $\mathrm{N}_{2}$ ó $\mathrm{N}_{2} \mathrm{O}$. La radiólisis de 
$\mathrm{CH}_{3} \mathrm{OH}$ y de mezclas $\mathrm{CH}_{3} \mathrm{OH} / \mathrm{H}_{2} \mathrm{O}$ han sido informadas en literatura y pueden ser utilizadas como una fuente de $e_{\text {solv }}^{-}$y radicales $\mathrm{C}^{\circ} \mathrm{H}_{2} \mathrm{OH}$, generados de acuerdo al siguiente esquema de reacción:

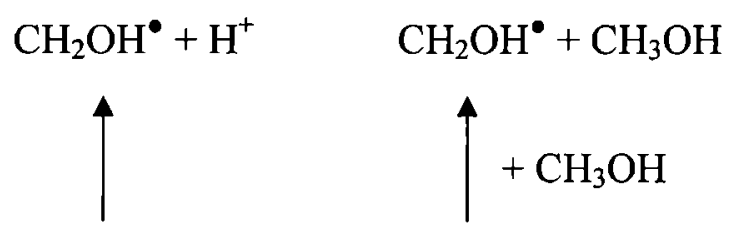

$$
\begin{gathered}
\mathrm{CH}_{3} \mathrm{OH}^{+}+\mathrm{e}_{\text {solv }}^{-}+\mathrm{CH}_{3} \mathrm{O}^{\bullet}+\mathrm{H}^{+} \\
\downarrow+\mathrm{CH}_{3} \mathrm{OH}+\mathrm{N}_{2} \mathrm{O} \\
\mathrm{CH}_{2} \mathrm{OH}^{\bullet}+\mathrm{N}_{2}+\mathrm{OH}^{-}
\end{gathered}
$$

Los potenciales de reducción versus electrodo normal de hidrógeno (ENH) del $e_{\text {solv }}^{-}$y del radical $\mathrm{C}^{\bullet} \mathrm{H}_{2} \mathrm{OH}$ se muestran en la taba 2 .

\begin{tabular}{|l|l|}
\hline Radical & $\begin{array}{l}\text { Potencial de } \\
\text { reducción / V } \\
\text { vs ENH }\end{array}$ \\
\hline$e_{\text {solv }}^{-}$ & -2.8 \\
\hline $\mathrm{C}^{\circ} \mathrm{H}_{2} \mathrm{OH}$ & -0.92 \\
\hline
\end{tabular}

Tabla 2: Potenciales de reducción de las especies $e_{\text {solv }}^{-}$y $\mathrm{C}^{\bullet} \mathrm{H}_{2} \mathrm{OH}$ vs ENH 
El rendimiento de $e_{\text {solv }}^{-}$en $\mathrm{CH}_{3} \mathrm{OH}$ es $\mathrm{G} \sim 1.1$ aproximadamente un tercio menos que el G-valor en la radiólisis del $\mathrm{H}_{2} \mathrm{O}, \mathrm{G} \sim 2.8$.

Cuando las soluciones son deaereadas con $\mathrm{N}_{2} \mathrm{O}$ el $e_{\text {solv }}^{-}$reacciona con el $\mathrm{N}_{2} \mathrm{O}$ (esquema 2) dejando como producto predominante al radical $\mathrm{C}^{\circ} \mathrm{H}_{2} \mathrm{OH}$ con un rendimiento $>90 \%$.

Con el fin de irradiar siempre muestra fresca, se utilizó un flujo de solución durante todos los experimentos.

Los experimentos de radiólisis de pulso fueron realizados en el Radiation Laboratory de la Universidad de Notre Dame, Indiana, Estados Unidos. El equipo utilizado fue un acelerador lineal de electrones modelo TB-8/16-1S, y se muestra en las figuras 1 y 2. Los datos fueron registrados por espectroscopía UV-visible resuelta en el tiempo. 


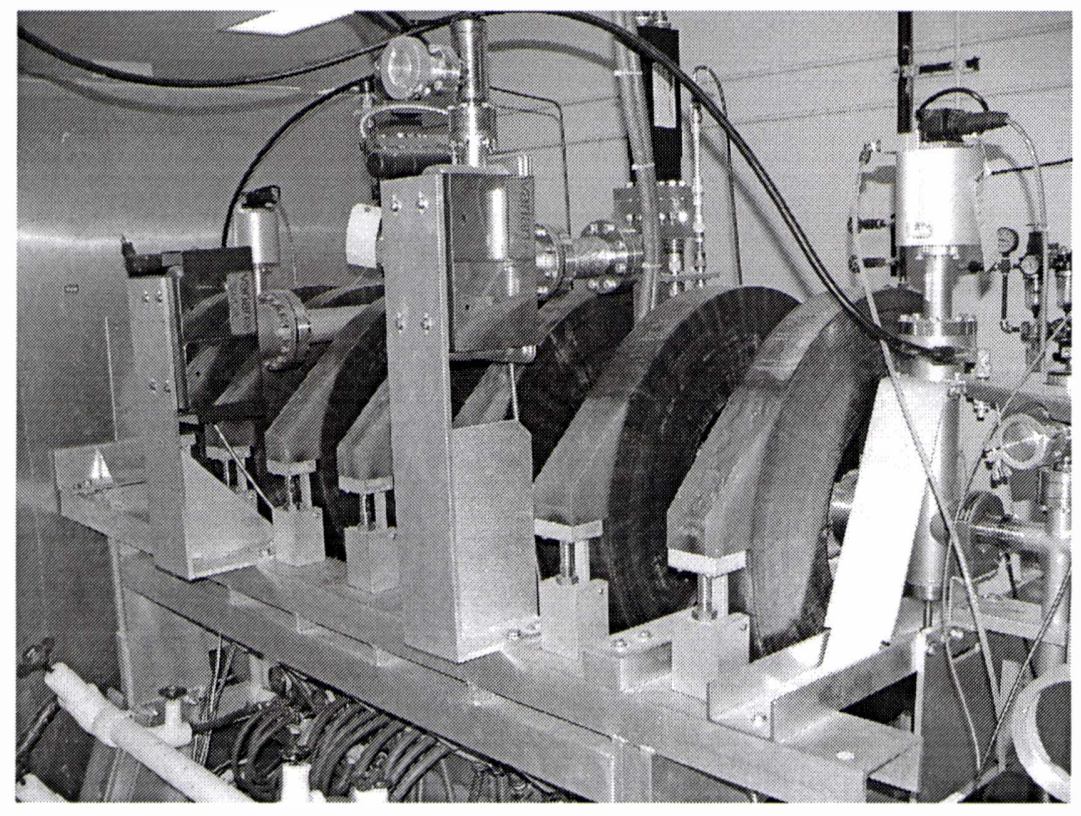

Figura 1: Acelerador lineal de electrones

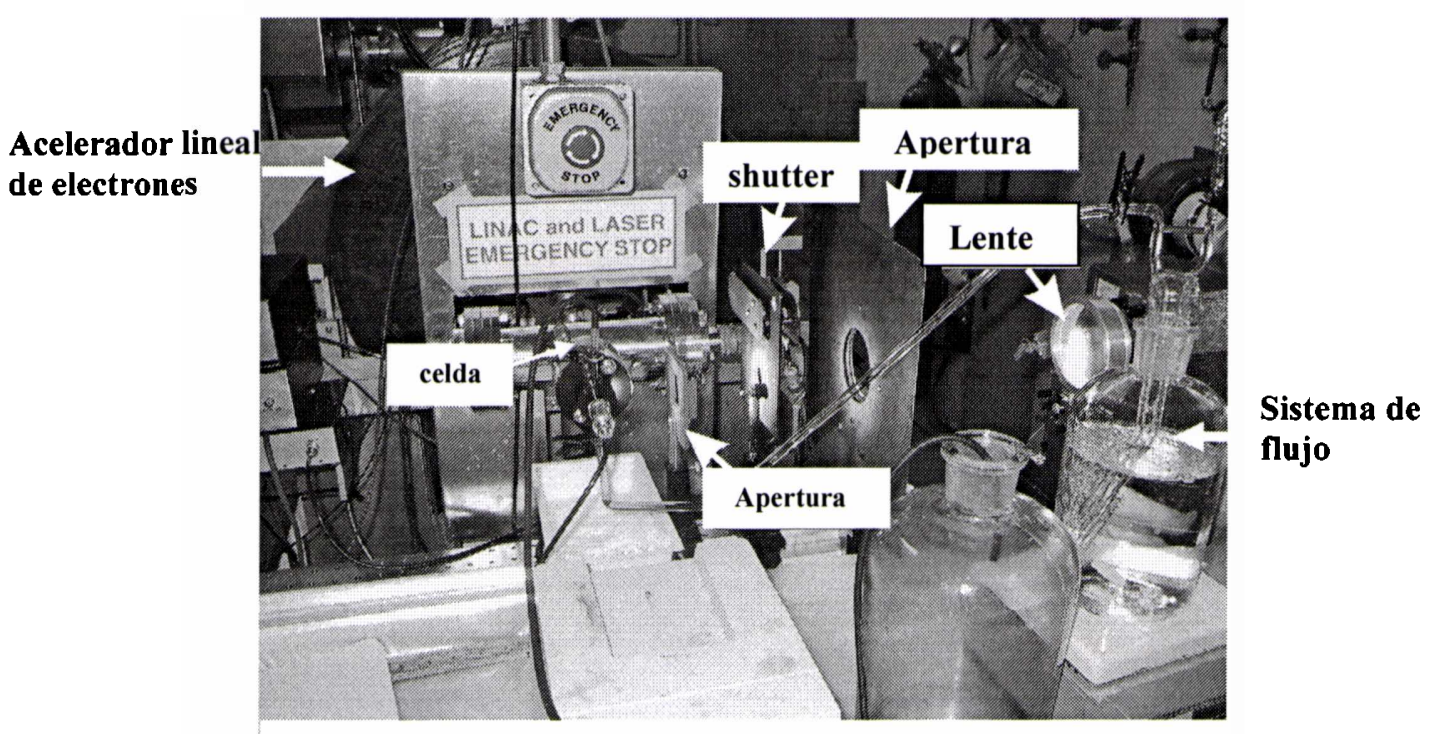

Figura 2: Foto del sistema de flujo, celda y sistema de lentes para el análisis de la absorción de las especies generadas con el pulso radiolítico 


\section{Bibliografía consultada en la Parte I}

1. Wolcan, E. Traabajo de tesis doctoral, Universidad Nacional de La Plata. 1996

2. Ruiz, G. T. Trabajo de tesis doctoral, Universidad Nacional de La Plata. 2003

3. Juliarena, M. P. Trabajo de tesis doctoral, Universidad Nacional de La Plata. 2005

4. Hoffman, M. Z. "Inorganic Photochemistry-State of the Art". J. Chem. Educ. 1983, Vol 60, Nro. $10,784-887$

5. Julliard M. and Chanon M. Chem. Rev. 1983, 83, 425-508

6. Demas, J. N. "Phophysical Pathways in Matal Complexes". J. Chem. Educ. 1983. Vol 60 , Nro. $10,803-808$

7. Porter, G. B. "Introduction to Inorganic Photochemistry". J. Chem. Educ. 1983, Vol 60, Nro. 10, 785-790

8. Crosby, G. A. "Structure, Bonding and Excited States of Coordination Complexes". J. Chem. Educ. 1983, Vol 60, Nro 10

9. Wrighton, M. S. Morse, D. L. J. Am. Chem. Soc. 1974, 96, 998

10. Wrighton, M. S. Morse, D. L. Pdungsap. J. Am. Chem. Soc. 1975, 97, 2073

11. Giordano, P. J. Wrighton, M. S. J. Am. Chem. Soc. 1979, 101, 2888

12. Lacowicz, J. R. “Principles of Fluorexcence Spectroscopy” Plenum Press Ed, New York, 1983

13. Ferraudi, G. J, "Elements of Inorganic Photochemistry" Ed. John Wiley and Sons, New York, 1988

14. Scaiano, J. C. "Handbook of Organic Photochemistry" Vol 1. CRC Press, Boca Raton, 1989 
15. Kalyanasundaram, K. and Gratzei, M. "Photosensitization and Photocatalysis Using Inorganic and Organometalic Compounds". Kluwer Academic Publishers, Dordrecht. 1993

16. Stufkens D. J. and Vicek Jr. A. Coord. Chem. Rev. 1998, 177, 127-179

17. Fox, M. A. and Chanon, M. "Photoinduced Electron Transfer. Part A and B Experimental Techniques and Medium Effects" Elsevier Science Publishers B. V.. Amsterdam, 1988

18. Turro, N. "Molecular Photochemistry" W. A. Benjamín, Inc. 1965

19. Demas, J. N. "Excited States Lifetime Measurements" Academic Press, New York. 1983 


\section{Parte II}

Polímeros derivatizados con complejos del tipo $\underline{\operatorname{Re}(\mathrm{CO})_{3} \mathbf{L}^{+} ; \mathbf{L}=\text { Azina bidentada }}$ 
Capítulo I

Síntesis y caracterización de polímeros de $\operatorname{Re}^{\mathrm{I}}$ 


\section{Capitulo I}

\section{Sintesis de $\left\{(v p y)_{2} v p y \operatorname{Re}(\mathrm{CO})_{3} L^{+}\right\}_{n-200}$}

Para la síntesis de los polímeros $\left\{(\mathrm{vpy})_{2} \mathrm{vpyRe}(\mathrm{CO})_{3}(\text { tmephen })^{+}\right\}_{\mathrm{n} \sim 200} \mathrm{y}$ $\left\{(\mathrm{vpy})_{2} \mathrm{vpyRe}(\mathrm{CO})_{3}\left(\mathrm{NO}_{2} \text {-phen }\right)^{+}\right\}_{\mathrm{n}-200}$. Esquema 1

Se procedió a la derivatización de la poli4-vinilpiridina (vpy) 600 PM: 60000 (Aldrich) con el cromóforo de $\mathrm{Re}^{1}$ correspondiente, que fue sintetizado siguiendo procedimientos descriptos en literatura. ${ }^{1-2}$

El compuesto de partida fue el Cloro-pentacarbonilo de $\operatorname{Re}^{\mathrm{I}}, \mathrm{Cl} \operatorname{Re}(\mathrm{CO})_{5}$, siendo el primer paso de síntesis la sustitución de dos grupos carbonilo, $\mathrm{CO}$, por el ligando bidentado ${ }^{3-4}$ correspondiente (tmephen ó $\mathrm{NO}_{2}$-phen), de acuerdo a la siguiente reacción:

$$
\mathrm{CIRe}(\mathrm{CO})_{5}+\mathrm{L}(20 \% \text { exceso }) \stackrel{60^{\circ} \mathrm{C}}{\longrightarrow} \operatorname{CIRe}(\mathrm{CO})_{3} \mathrm{~L}+2 \mathrm{CO}
$$

La reacción se realizó en isooctano como solvente, calentando a reflujo y con agitación durante 6 horas.

El producto obtenido resultó ser un precipitado amarillo, que fue separado de la solución por filtración.

Para su purificación el producto se recristalizó, disolviendo el sólido en la mínima cantidad de diclorometano, $\mathrm{CH}_{2} \mathrm{Cl}_{2}, \mathrm{y}$ precipitándolo por agregado, gota a gota, de isooctano. Este procedimiento se repitió hasta obtener valor constante del coeficiente de extinción molar (E). El sólido asi obtenido se secó hasta peso constante.

El siguiente paso de síntesis, fue la sustitución de cloruro, $\mathrm{Cl}^{-}$, por el ión triflato, $\mathrm{CF}_{3} \mathrm{SO}_{3}^{-}$, para lo cual se hizo reaccionar el producto anteriormente obtenido con triflato de plata, $\mathrm{CF}_{3} \mathrm{SO}_{3} \mathrm{Ag}$, según la siguiente reacción: 


\section{$\mathrm{ClRe}(\mathrm{CO})_{3} \mathrm{~L}+\mathrm{CF}_{3} \mathrm{SO}_{3} \mathrm{Ag} \rightarrow \mathrm{CF}_{3} \mathrm{SO}_{3} \mathrm{Re}(\mathrm{CO})_{3} \mathrm{~L}+\mathrm{ClAg} \downarrow$}

La reacción se llevó a cabo en tolueno, calentando a reflujo y agitando durante toda la noche.

El precipitado de ClAg fue eliminado por filtración quedando el producto deseado en solución de la cual fue recuperado por rotoevaporación del solvente.

Para la purificación del producto se realizó una recristalización, según el procedimiento anteriormente descripto con diclorometano / isooctano hasta obtener valor constante del $\varepsilon$. El sólido amarillo obtenido, fue secado hasta peso constante.

El último paso de síntesis fue la derivatización de la poli4-vinilpiridina comercial, (vpy) $)_{600}$, con el $\mathrm{CF}_{3} \mathrm{SO}_{3} \mathrm{Re}(\mathrm{CO})_{3} \mathrm{~L}$ correspondiente, las cantidades de cada reactivo fueron tales que en promedio quedan 200 grupos pendientes por mol de polímero, es decir cada 600 piridinas aproximadamente. ${ }^{1}$ La reacción ocurre, según:

$$
(\mathrm{vpy})_{600}+\mathrm{CF}_{3} \mathrm{SO}_{3} \operatorname{Re}(\mathrm{CO})_{3} \mathrm{~L} \rightarrow\left\{(\mathrm{vpy})_{2} \mathrm{vpyRe}(\mathrm{CO})_{3} \mathrm{~L}\right\}_{200}\left(\mathrm{CF}_{3} \mathrm{SO}_{3}\right)_{200}
$$

La misma se realizó en $\mathrm{CH}_{2} \mathrm{CL}_{2}$ calentando a reflujo y con agitación durante 6 horas.

El polímero derivatizado fue soluble, por lo tanto, se eliminó el solvente por rotoevaporación, y se realizó la purificación, por medio de una recristalización disolviéndolo en la mínima cantidad de acetonitrilo, $\mathrm{CH}_{3} \mathrm{CN}$, y precipitándolo por el agregado, gota a gota, de éter etílico, $\mathrm{CH}_{3} \mathrm{CH}_{2}-\mathrm{O}-\mathrm{CH}_{2} \mathrm{CH}_{3}$, el procedimiento se repitió hasta valor constante del $\varepsilon$. El sólido obtenido se secó hasta peso constante. 


\section{Sintesis de los polímeros $\left\{(v p y)_{2} v p y \operatorname{Re}(\mathrm{CO})_{3} L_{1}^{+}\right\}_{n}\left\{(v p y)_{2} v p y \operatorname{Re}(\mathrm{CO})_{3} L_{2}{ }^{+}\right\}_{m}$} $n+m=200$

Se sintetizaron los polímeros mixtos:

$\left\{(\mathrm{vpy})_{2} \mathrm{vpyRe}(\mathrm{CO})_{3}(\text { tmephen })^{+}\right\}_{\mathrm{n} \sim 180}\left\{(\mathrm{vpy})_{2} \mathrm{vpyRe}(\mathrm{CO})_{3}\left(\mathrm{NO}_{2}-\mathrm{phen}\right)^{+}\right\}_{\mathrm{m} \sim 20}$

$\left\{(\mathrm{vpy})_{2} \mathrm{vpyRe}(\mathrm{CO})_{3}(\text { tmephen })^{+}\right\}_{n \sim 150}\left\{(\mathrm{vpy})_{2} \mathrm{vpyRe}(\mathrm{CO})_{3}\left(\mathrm{NO}_{2}-\text { phen }\right)^{+}\right\}_{\mathrm{m} \sim 50}$

$\left\{(\text { vpy })_{2} \text { vpyRe }(\mathrm{CO})_{3}(\text { tmephen })^{+}\right)_{n \sim 100}\left\{(\text { vpy })_{2} \text { vpyRe }(\mathrm{CO})_{3}\left(\mathrm{NO}_{2} \text {-phen }\right)^{+}\right\}_{\mathrm{m} \sim 100}$. Esquema 2

Para ello se derivatizó la (vpy) 600 con las mezclas molares de,

$\mathrm{CF}_{3} \mathrm{SO}_{3} \mathrm{Re}(\mathrm{CO})_{3} \mathrm{~L}_{1} / \mathrm{CF}_{3} \mathrm{SO}_{3} \mathrm{Re}(\mathrm{CO})_{3} \mathrm{~L}_{2}$ necesarias para obtener la relación $\mathrm{L}_{1} / \mathrm{L}_{2}$ deseada, tmephen / $\mathrm{NO}_{2}$-phen: $180 / 20 ; 150 / 50 ; 100 / 100$ de manera tal que en promedio sean 200 los grupos pendientes por cada mol de polimero (600 piridinas).

La reacción general es:

$\mathrm{CF}_{3} \mathrm{SO}_{3} \operatorname{Re}(\mathrm{CO})_{3} \mathrm{~L}_{1}+\mathrm{CF}_{3} \mathrm{SO}_{3} \operatorname{Re}(\mathrm{CO})_{3} \mathrm{~L}_{2}+(\mathrm{vpy})_{600} \rightarrow$

$\left\{\left(\mathrm{vpy}_{2} \mathrm{vpyRe}^{+}(\mathrm{CO})_{3} \mathrm{~L}_{1}\right\}_{\mathrm{n}}\left\{(\mathrm{vpy})_{2} \mathrm{vpyRe}^{+}(\mathrm{CO})_{3} \mathrm{~L}_{2}\right\}_{\mathrm{m}}\right.$

Se realizó en $\mathrm{CH}_{2} \mathrm{Cl}_{2}$ calentando a reflujo durante 9 horas.

Debido a la solubilidad del producto obtenido fue necesario rotoevaporar para eliminar el solvente. Finalmente se recristalizó con el sistema acetonitrilo / éter etilíco hasta valor constante de $\varepsilon$. El sólido obtenido se secó hasta peso constante. Este procedimiento fue utilizado para los tres polímeros mixtos. 

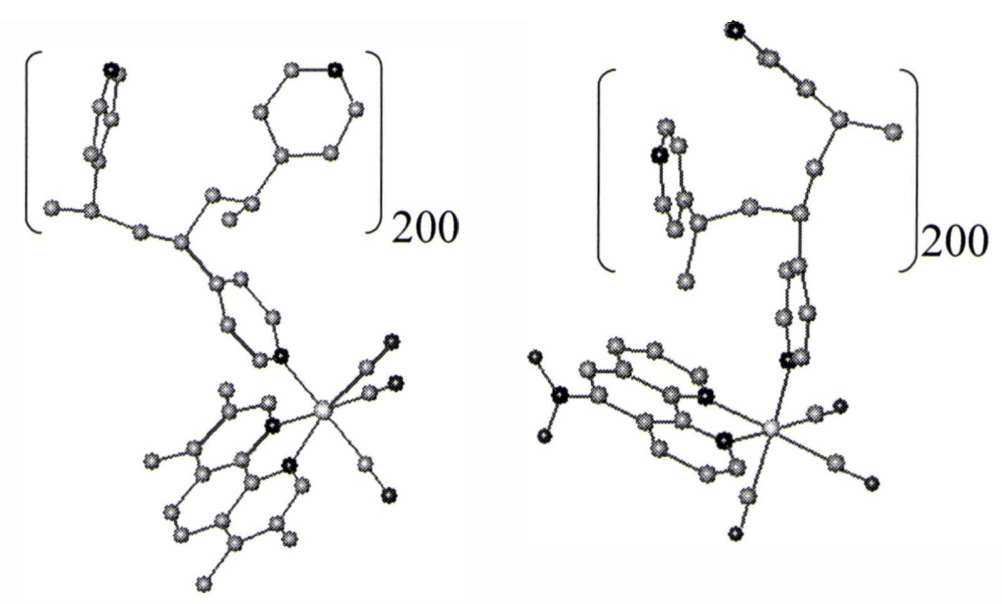

\section{Esquema I}

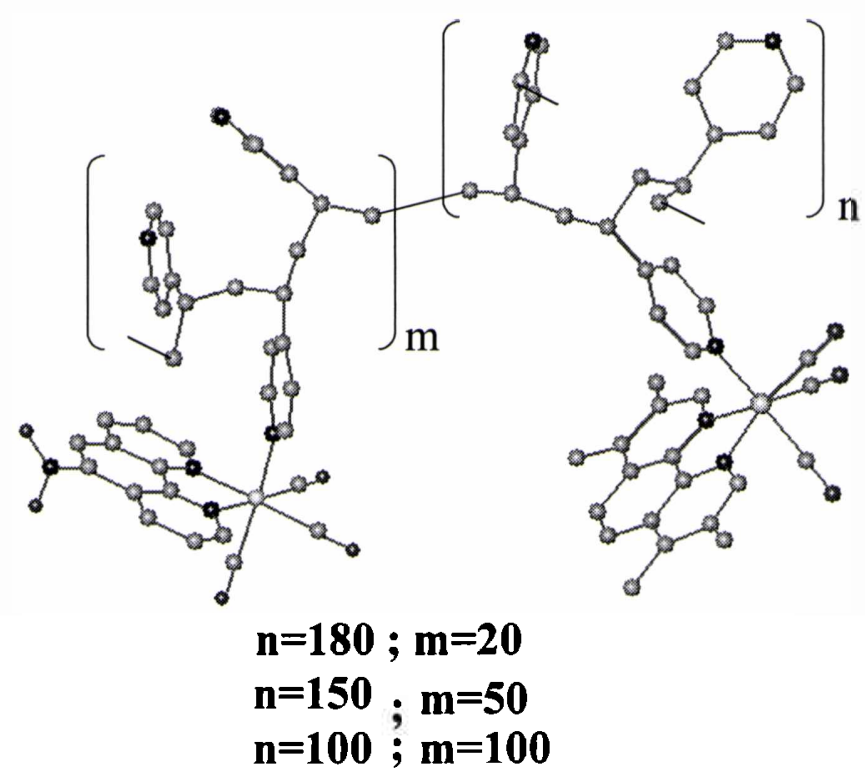

Esquema 2 


\section{Caracterización de los polímeros sintetizados}

Con el fin de caracterizar los productos se realizaron: análisis elemental, espectroscopía UV-Visible y espectroscopía FT-IR. Además se estudió la morfología de estos cinco polímeros en films mediante microscopía electrónica de transmisión (TEM)

\section{i. Análisis elemntal}

Los resultados del análisis elemental se muestran en la tabla 1:

\begin{tabular}{|c|c|c|c|c|c|c|}
\hline Polímero & Teórico & & & Calcula & & \\
\hline & $\mathrm{C}$ & $\bar{N}$ & $\mathrm{H}$ & C & $\mathrm{N}$ & $\mathrm{H}$ \\
\hline$\left\{(\mathrm{vpy})_{2} \operatorname{Re}(\mathrm{CO})_{3}\left(\mathrm{NO}_{2}-\mathrm{phen}\right)+\right\}_{n-200}$ & 50.21 & 6.82 & 3.82 & 50.26 & 7.12 & 3.80 \\
\hline$\left\{(\text { vpy })_{2} \text { vpyRe}(\mathrm{CO})_{3}(\text { tmephen })+\right\}_{n-200}$ & 46.02 & 8.48 & 3.35 & 45.76 & 8.68 & 2.90 \\
\hline$\left\{(\text { vpy })_{2} \text { vpyRe }(\mathrm{CO})_{3}(\text { tmephen })+\right\}_{n \sim 180}\left\{(\mathrm{vpy})_{2} \mathrm{vpyRe}(\mathrm{CO})_{3}\left(\mathrm{NO}_{2}-\text { phen }\right)+\right\}_{\mathrm{m} \sim 20}$ & 49.50 & 6.15 & 4.49 & 49.81 & 7.27 & 3.70 \\
\hline$\left\{(\mathrm{vpy})_{2} \mathrm{vpyRe}(\mathrm{CO})_{3}(\mathrm{tmephen})+\right\}_{n \sim 150}\left\{(\mathrm{vpy})_{2} \mathrm{vpyRe}(\mathrm{CO})_{3}\left(\mathrm{NO}_{2}-\text { phen }\right)+\right\}_{\mathrm{m} \sim 50}$ & 49.35 & 7.09 & 3.85 & 49.14 & 7.5 & 3.60 \\
\hline$\left\{(\mathrm{vpy})_{2} \mathrm{vpyRe}(\mathrm{CO})_{3}(\text { tmephen })+\right)_{n-100}\left\{(\mathrm{vpy})_{2} \mathrm{vpyRe}(\mathrm{CO})_{3}\left(\mathrm{NO}_{2}-\mathrm{phen}\right)+\right\}_{m \sim 100}$ & 48.01 & 7.90 & 3.34 & 47.86 & 7.91 & 3.50 \\
\hline
\end{tabular}

Tabla 1: Análisis elemental de los cinco polímeros sintetizados. Comparacion entre los valores teóricos y calculados.

\section{ii. Espectroscopía de absorción UV-Visible}

En la figura 1 se muestran los espectros UV-Visible de los polímeros:

$\left\{(\mathrm{vpy})_{2} \mathrm{vpyRe}(\mathrm{CO})_{3}(\text { tmephen })^{+}\right\}_{\mathrm{n} \sim 200} ;\left\{(\mathrm{vpy})_{2} \mathrm{vpyRe}(\mathrm{CO})_{3}\left(\mathrm{NO}_{2}-\mathrm{phen}\right)^{+}\right\}_{\mathrm{n} \sim 200} ;$

$\left\{(\text { vpy })_{2} \operatorname{vpyRe}(\mathrm{CO})_{3}(\text { tmephen })^{+}\right\}_{\mathrm{n} \sim 180}\left\{(\mathrm{vpy})_{2} \mathrm{vpyRe}(\mathrm{CO})_{3}\left(\mathrm{NO}_{2} \text {-phen }\right)^{+}\right\}_{\mathrm{m} \sim 20}$

$\left\{(\mathrm{vpy})_{2} \mathrm{vpyRe}(\mathrm{CO})_{3}(\text { tmephen })^{+}\right\}_{\mathrm{n} \sim 50}\left\{(\mathrm{vpy})_{2} \mathrm{vpyRe}(\mathrm{CO})_{3}\left(\mathrm{NO}_{2}-\text { phen }\right)^{+}\right\}_{\mathrm{m} \sim 50} \mathrm{y}$ 
$\left\{(\text { vpy })_{2} \text { vpyRe}(\mathrm{CO})_{3}(\text { tmephen })^{+}\right)_{\mathrm{n} \sim 100}\left\{(\mathrm{vpy})_{2} \mathrm{vpyRe}(\mathrm{CO})_{3}\left(\mathrm{NO}_{2}-\text { phen }\right)^{+}\right\}_{\mathrm{m} \sim 100}$

Dichos espectros fueron registrados en un espectrofotómetro CARY 3, sobre soluciones preparadas en $\mathrm{CH}_{3} \mathrm{CN}$ como solvente y a temperatura ambiente.

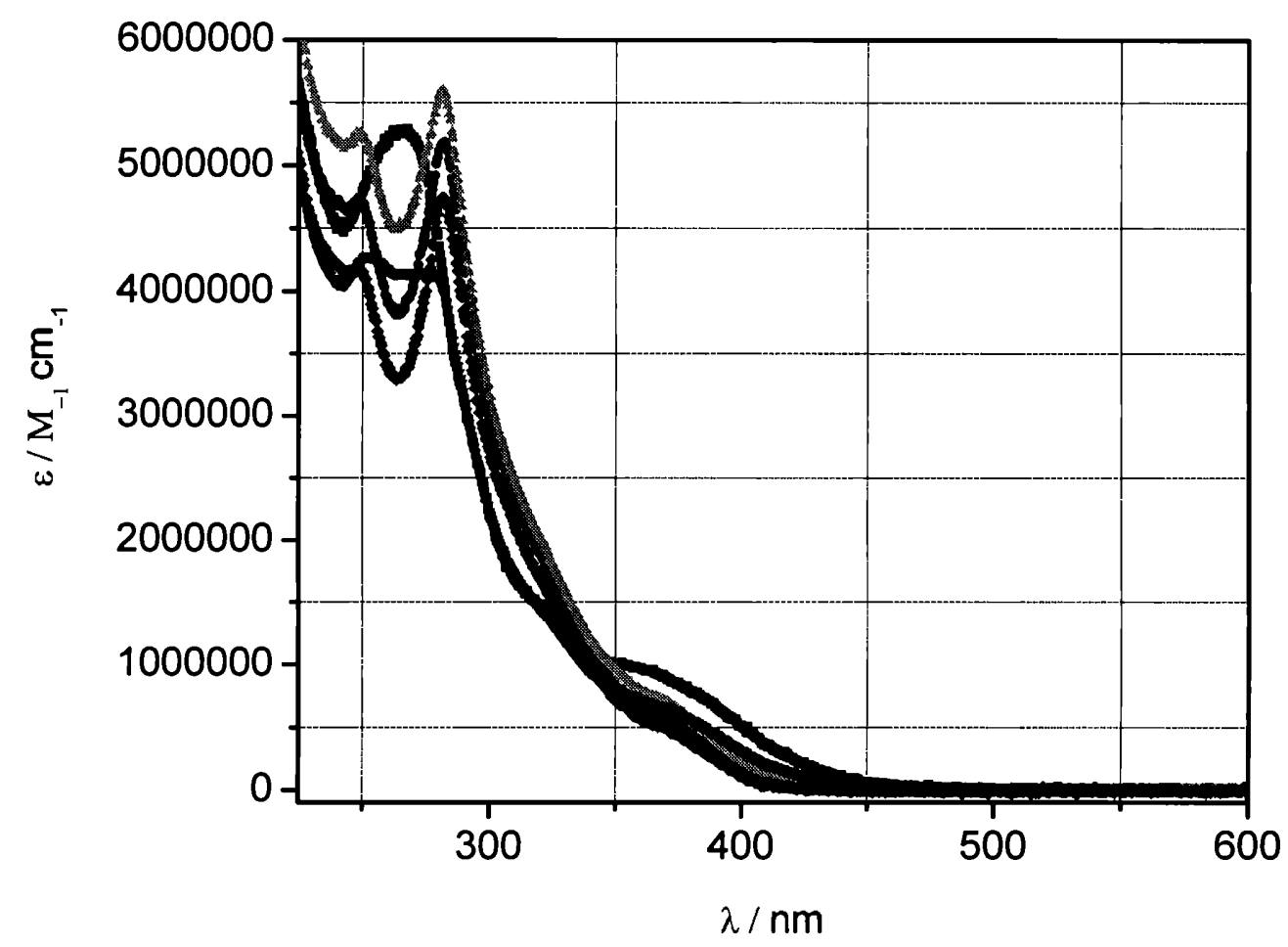

Figura 1: Espectro de absorción de los polimeros $\left\{(\mathrm{vpy})_{2} \mathrm{vpyRe}(\mathrm{CO})_{3} \mathrm{~L}_{1}{ }^{+}\right\}_{n}\left\{(\mathrm{vpy})_{2} \mathrm{vpyRe}(\mathrm{CO})_{3} \mathrm{~L}_{2}{ }^{+}\right\}_{\mathrm{m}}$ en $\mathrm{CH}_{3} \mathrm{CN}$ a temperatura ambiente

- $\left\{(\mathrm{vpy})_{2} \mathrm{vpyRe}(\mathrm{CO})_{3}\left(\mathrm{NO}_{2}-\mathrm{phen}\right)^{+}\right\}_{\mathrm{n} \sim 200}$,

- $\left.\left\{(\text { vpy })_{2} \text { vpyRe }(\mathrm{CO})_{3}(\text { tmephen })^{+}\right\}_{n-180}\left\{(\mathrm{vpy})_{2} \text { vpyRe(CO) }\right)_{3}\left(\mathrm{NO}_{2}-\text { phen }\right)^{+}\right\}_{\mathrm{m} \sim 20}$,

$\downarrow\left\{(\mathrm{vpy})_{2} \mathrm{vpyRe}(\mathrm{CO})_{3}(\text { tmephen })^{+}\right\}_{\mathrm{n} \sim 150}\left\{(\mathrm{vpy})_{2} \mathrm{vpyRe}(\mathrm{CO})_{3}\left(\mathrm{NO}_{2}-\mathrm{phen}\right)^{+}\right\}_{\mathrm{m} \sim 50}$,

$\nabla\left\{(\text { vpy })_{2} \text { vpyRe }(\mathrm{CO})_{3}(\text { tmephen })^{+}\right)_{n \sim 100}\left\{(\text { vpy })_{2} \text { vpyRe }(\mathrm{CO})_{3}\left(\mathrm{NO}_{2} \text {-phen }\right)^{+}\right\}_{\mathrm{m} \sim 100}$,

- $\left\{(\mathrm{vpy})_{2} \mathrm{vpy} \operatorname{Re}(\mathrm{CO})_{3}(\text { tmephen })^{+}\right\}_{\mathrm{n}-200}$

La asignación de bandas se muestra en la tabla 2 
Tabla 2: Asignación de transiciones a las bandas observadas en los espectros de absorción de los polímeros derivatizados con complejos de $\mathbf{R e}^{1}$

Polímero $\lambda / \mathrm{nm}$

Asignación

$\left.\overline{\{(\mathrm{vpy}}_{2} \mathrm{vpyRe}(\mathrm{CO})_{3}(\mathrm{tmephen})^{+}\right\}_{\mathrm{n}-200}$

248

282

325

369

267

$\left\{(\mathrm{vpy})_{2} \mathrm{vpyRe}(\mathrm{CO})_{3}\left(\mathrm{NO}_{2} \text {-phen }\right)^{+}\right\}_{n \sim 200}$

$\left\{(\mathrm{vpy})_{2} \mathrm{vpyRe}(\mathrm{CO})_{3}(\text { tmephen })^{+}\right\}_{\mathrm{n} \sim 180}\left\{(\mathrm{vpy})_{2} \mathrm{vpyRe}(\mathrm{CO})_{3}\left(\mathrm{NO}_{2}-\mathrm{phen}\right)^{+}\right\}_{\mathrm{m} \sim 20}$

$\left\{(\mathrm{vpy})_{2} \mathrm{vpyRe}(\mathrm{CO})_{3}(\text { tmephen })^{+}\right\}_{\mathrm{n} \sim 150}\left\{(\mathrm{vpy})_{2} \mathrm{vpyRe}(\mathrm{CO})_{3}\left(\mathrm{NO}_{2}-\text { phen }\right)^{+}\right\}_{\mathrm{m} \sim 50}$

$\left\{(\mathrm{vpy})_{2} \mathrm{vpyRe}(\mathrm{CO})_{3}(\text { tmephen })^{+}\right)_{\mathrm{n} \sim 100}\left\{(\mathrm{vpy})_{2} \mathrm{vpyRe}(\mathrm{CO})_{3}\left(\mathrm{NO}_{2}-\mathrm{phen}\right)^{+}\right\}_{\mathrm{m} \sim 100}$

$\begin{array}{ll}324 & \pi \rightarrow \pi^{*} \\ 359 & \mathrm{~d}_{\pi} \rightarrow \pi^{*} \\ 389 & \end{array}$

248

$\mathrm{d}_{\pi} \rightarrow \pi^{*}$

$\mathrm{d}_{\pi} \rightarrow \pi^{*}$

252

$\mathrm{d}_{\pi} \rightarrow \pi^{*}$ 


\section{iii. Microscopía electrónica de transmisión. TEM}

La microscopía electrónica de transmisión fue realizada con un microscopio JEOL $100 \mathrm{CX}$ con un voltaje de aceleración de electrón de $80 \mathrm{Kv}$ y con una resolución nominal, punto a punto de $3 \AA$.

Se prepararon soluciones de los cinco polímeros sintetizados en los solventes acetonitrilo y diclorometano, respectivamente. Para la obtención de los films es necesario evaporar el solvente, a temperatura ambiente.

Los films no fueron teñidos con ningún químico, y el contraste de la imagen en las fotos es originado sólo por los complejos de Re $\mathrm{I}^{\mathrm{I}}$ presentes en el polímero.

Las fotos fueron ampliadas $20000 \mathrm{x}$, y se muestran en las figuras 2 y 3 respectivamente 


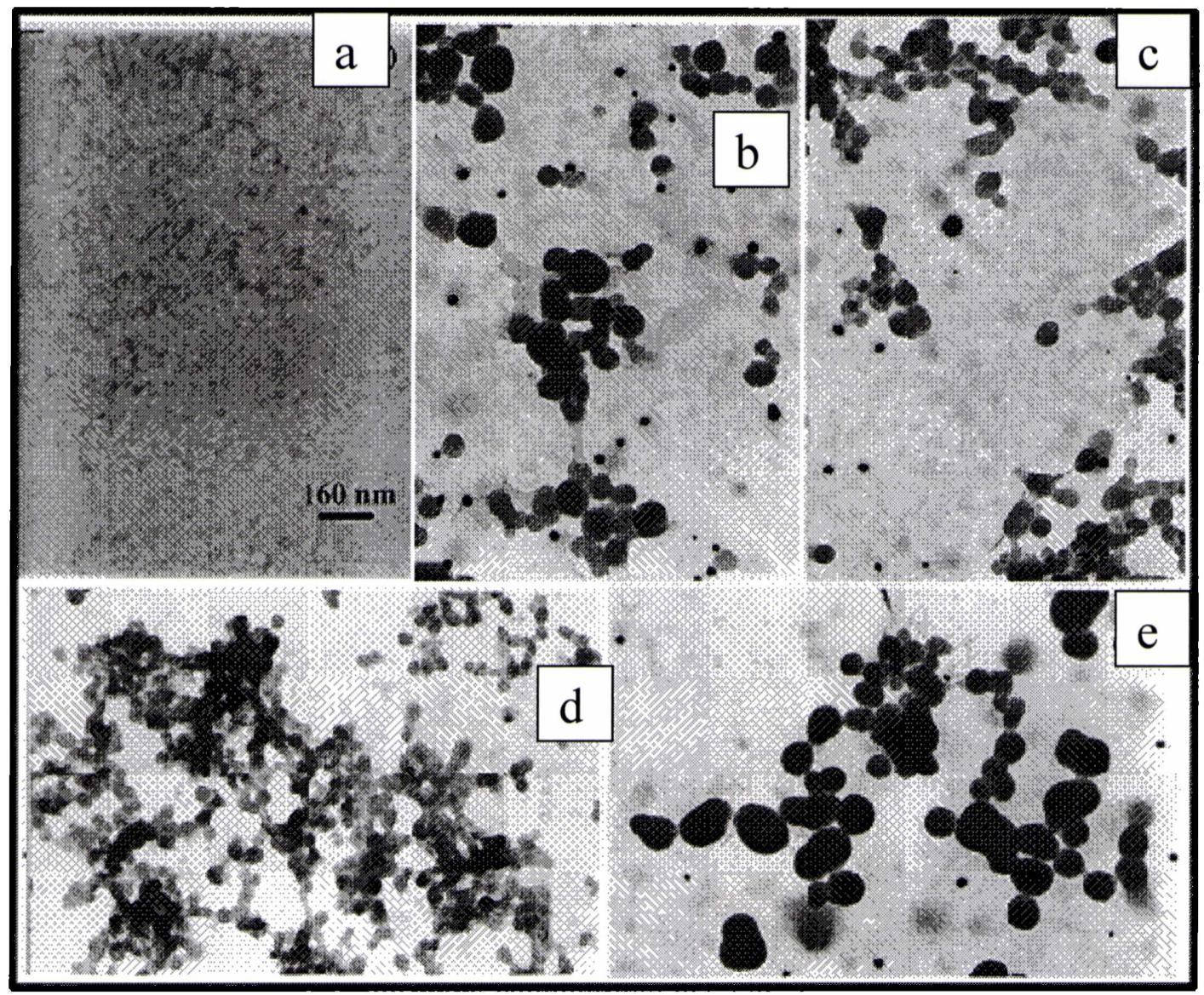

Figura 2: Films obtenidos a partir de soluciones en $\mathrm{CH}_{3} \mathrm{CN}$ de los polímeros
a: $\left\{(\mathrm{vpy})_{2} \mathrm{vpy} \operatorname{Re}(\mathrm{CO})_{3}(\text { tmephen })^{+}\right\}_{\mathrm{n}-200} ;$ b: $\left\{(\mathrm{vpy})_{2} \mathrm{vpyRe}(\mathrm{CO})_{3}\left(\mathrm{NO}_{2}-\mathrm{phen}\right)^{+}\right\}_{\mathrm{n}-200}$;
c: $\left\{(\mathrm{vpy})_{2} \mathrm{vpy} \operatorname{Re}(\mathrm{CO})_{3}(\text { tmephen })^{+}\right\}_{\mathrm{n} \sim 180}\left\{(\mathrm{vpy})_{2} \mathrm{vpy} \operatorname{Re}(\mathrm{CO})_{3}\left(\mathrm{NO}_{2}-\mathrm{phen}\right)^{+}\right\}_{\mathrm{m} \sim 20}$;
$\mathrm{d}:\left\{(\mathrm{vpy})_{2} \mathrm{vpyRe}(\mathrm{CO})_{3}(\text { tmephen })^{+}\right\}_{\mathrm{n} \sim 150}\left\{(\mathrm{vpy})_{2} \mathrm{vpyRe}(\mathrm{CO})_{3}\left(\mathrm{NO}_{2}-\text { phen }\right)^{+}\right\}_{\mathrm{m} \sim 50} \mathrm{y}$
e: $\left\{(\mathrm{vpy})_{2} \mathrm{vpyRe}(\mathrm{CO})_{3}(\text { tmephen })^{+}\right)_{n \sim 100}\left\{(\mathrm{vpy})_{2} \mathrm{vpyRe}(\mathrm{CO})_{3}\left(\mathrm{NO}_{2}-\text { phen }\right)^{+}\right\}_{\mathrm{m} \sim 100}$.

Fue aplicada la misma escala en todas las fotos. 


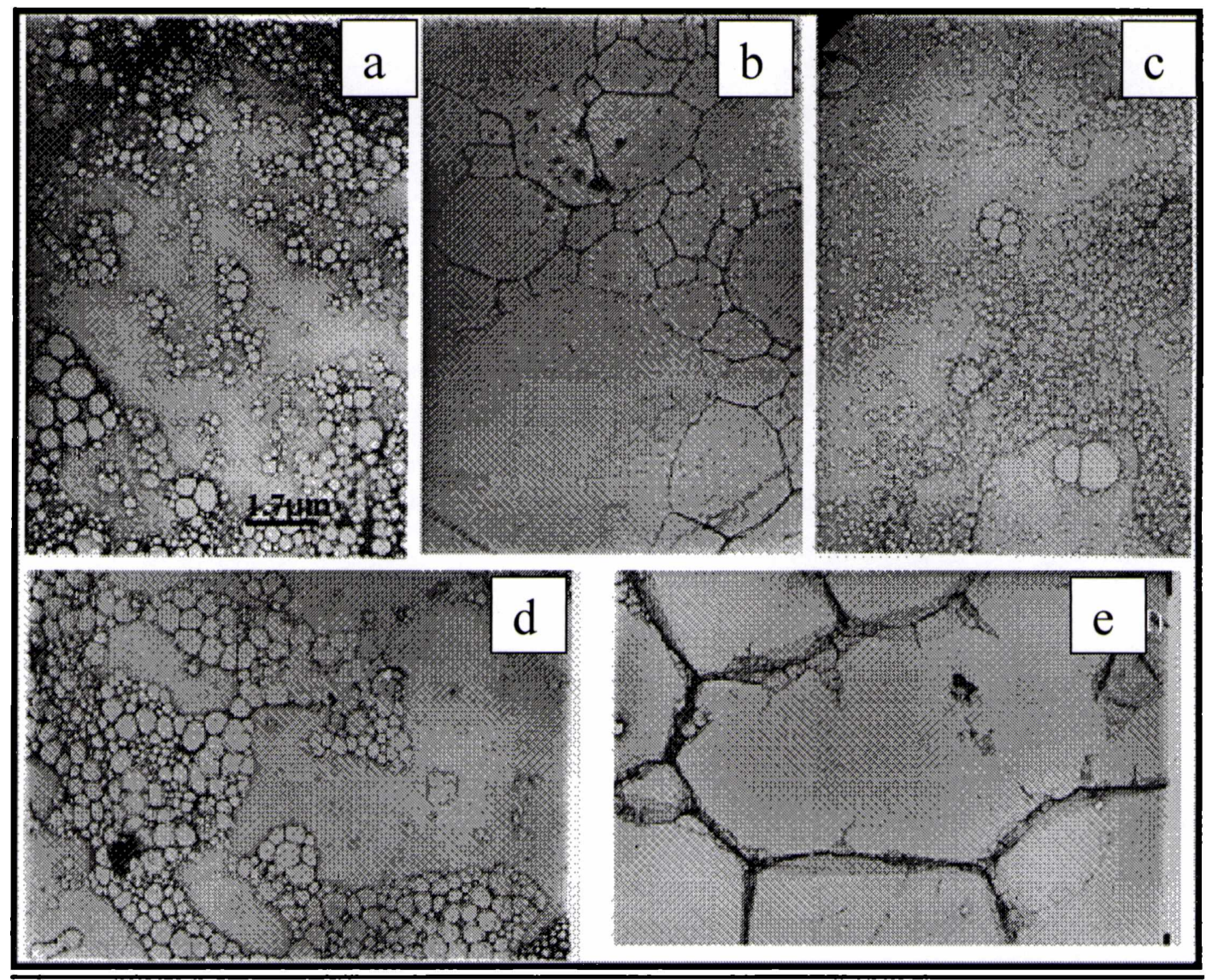

Figura 3: Films obtenidos a partir de soluciones en $\mathrm{CH}_{2} \mathrm{Cl}_{2}$ de los polímeros:

a: $\left\{(\mathrm{vpy})_{2} \mathrm{vpyRe}(\mathrm{CO})_{3}(\text { tmephen })^{+}\right\}_{n \sim 200} ;$ b: $\left\{(\mathrm{vpy})_{2} \mathrm{vpyRe}(\mathrm{CO})_{3}\left(\mathrm{NO}_{2}-\text { phen }\right)^{+}\right\}_{n \sim 200}$

c: $\left\{(\mathrm{vpy})_{2} \mathrm{vpyRe}(\mathrm{CO})_{3}(\mathrm{tmephen})^{+}\right\}_{\mathrm{n}-180}\left\{(\mathrm{vpy})_{2} \mathrm{vpyRe}(\mathrm{CO})_{3}\left(\mathrm{NO}_{2}-\mathrm{phen}\right)^{+}\right\}_{\mathrm{m}-20}$;

d: $\left\{(\mathrm{vpy})_{2} \mathrm{vpyRe}(\mathrm{CO})_{3}(\text { tmephen })^{+}\right\}_{\mathrm{n} \sim 150}\left\{(\mathrm{vpy})_{2} \mathrm{vpyRe}(\mathrm{CO})_{3}\left(\mathrm{NO}_{2}-\text { phen }\right)^{+}\right\}_{\mathrm{m} \sim 50} \mathrm{Y}$

e: $\left\{(\text { vpy })_{2} \text { vpyRe }(\mathrm{CO})_{3}(\text { tmephen })^{+}\right)_{n \sim 100}\left\{(\mathrm{vpy})_{2} \mathrm{vpyRe}(\mathrm{CO})_{3}\left(\mathrm{NO}_{2} \text {-phen }\right)^{+}\right\}_{\mathrm{m}-100}$.

Fue aplicada la misma escala en todas las fotos. 


\section{iv. Espectroscopia FT-IR}

Se realizaron los espectros infrarrojos de los cinco polímeros sintetizados. Para lo cual se utilizó la técnica de pastilla en $\mathrm{KBr}$.

Los resultados obtenidos se muestran en la tabla 3 y en las figuras 4 y 5 .

\begin{tabular}{|c|c|c|}
\hline Polimero & \multicolumn{2}{|c|}{$\mathrm{v}(\mathrm{CO}) / \mathrm{cm}^{-1}$} \\
\hline$\left\{(\mathrm{vpy})_{2} \operatorname{Re}(\mathrm{CO})_{3}\left(\mathrm{NO}_{2}-\mathrm{phen}\right)^{+}\right\}_{n-200}$ & 2034 & 1912 \\
\hline$\left\{(\text { vpy })_{2} \text { vpyRe }(\mathrm{CO})_{3}(\text { tmephen })^{+}\right\}_{n-200}$ & 2029 & 1902 \\
\hline$\left\{(\mathrm{vpy})_{2} \mathrm{vpyRe}(\mathrm{CO})_{3}(\mathrm{tmephen})^{+}\right\}_{n-180}\left\{(\mathrm{vpy})_{2} \mathrm{vpyRe}(\mathrm{CO})_{3}\left(\mathrm{NO}_{2}-\mathrm{phen}\right)^{+}\right\}_{m-20}$ & 2029 & 1902 \\
\hline$\left\{(\mathrm{vpy})_{2} \mathrm{vpyRe}(\mathrm{CO})_{3}(\text { tmephen })^{+}\right\}_{n-150}\left\{(\mathrm{vpy})_{2} \mathrm{vpyRe}(\mathrm{CO})_{3}\left(\mathrm{NO}_{2}-\text { phen }\right)^{+}\right\}_{m-50}$ & 2030 & 1906 \\
\hline$\left\{(\mathrm{VPy})_{2} \mathrm{VPy} \operatorname{Re}(\mathrm{CO})_{3}(\text { tmephen })^{+}\right)_{n-100}\left\{(\mathrm{VPy})_{2} \mathrm{VPy} \operatorname{Re}(\mathrm{CO})_{3}\left(\mathrm{NO}_{2} \text {-phen }\right)^{+}\right\}_{\mathrm{m}-10}$ & 2032 & 1907 \\
\hline
\end{tabular}

Tabla 3: Frecuencia de estiramiento del grupo $\mathrm{CO}, \mathrm{v}(\mathrm{CO}) / \mathrm{cm}^{-1}$, mostrando su corrimiento con el aumento del cromóforo $-\operatorname{Re}(\mathrm{CO})_{3}\left(\mathrm{NO}_{2} \text {-phen }\right)^{+}$relativo al $-\mathrm{Re}(\mathrm{CO})_{3}(\text { tmephen })^{+}$ 


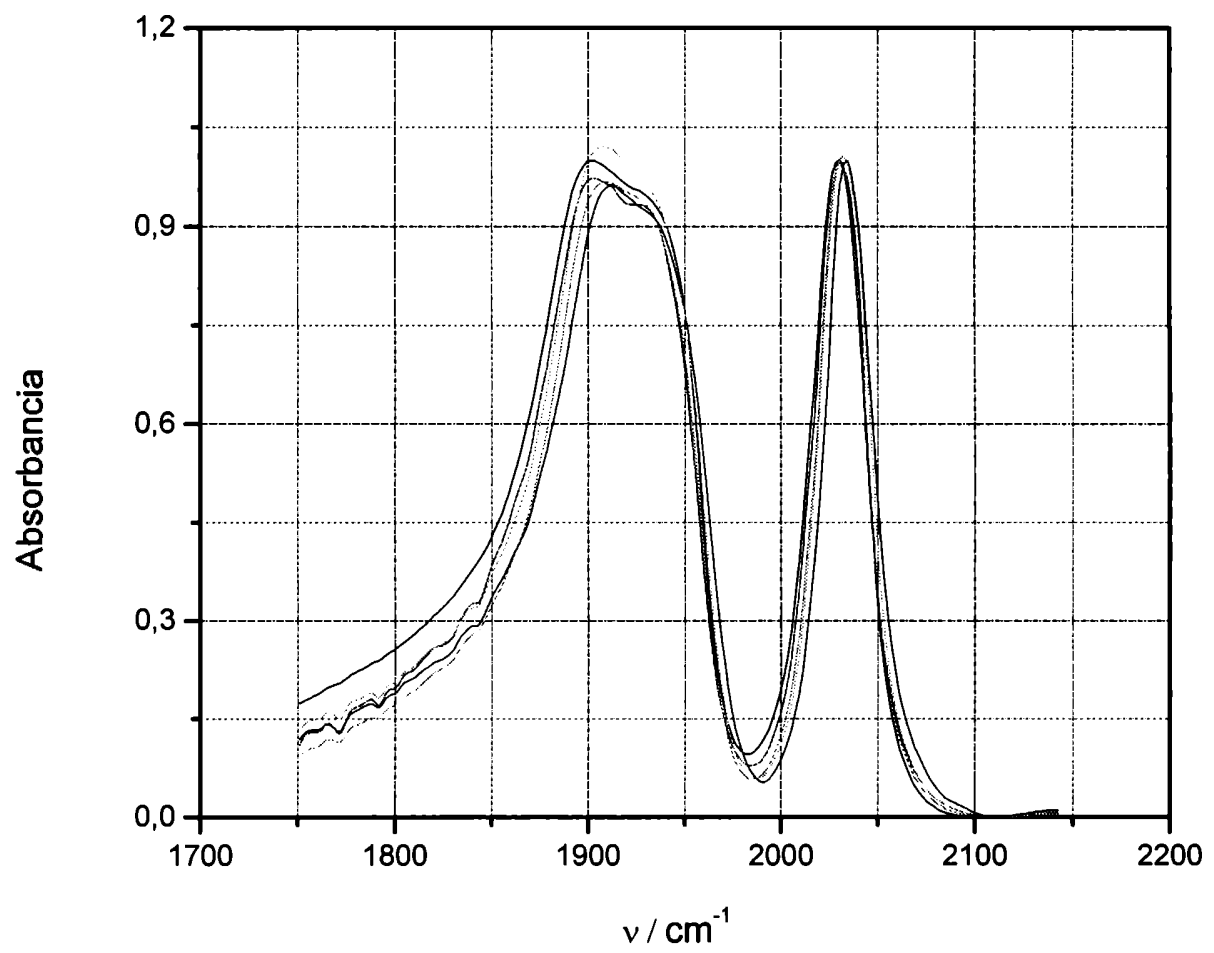

Figura 4: Espectro infrarrojo (IR) realizados en pellets de $\mathrm{KBr}$ mostrando los cambios en la Región de $\left.v(\mathrm{CO}) .-\left\{(\mathrm{vpy})_{2} \mathrm{vpyRe}(\mathrm{CO})_{3} \text { (tmephen }\right)^{+}\right\}_{\mathrm{n}-200}$

$-\left\{(\mathrm{vpy})_{2} \mathrm{vpyRe}(\mathrm{CO})_{3}\left(\mathrm{NO}_{2} \text {-phen }\right)^{+}\right\}_{n \sim 200}$

$\ldots-\left\{(\mathrm{vpy})_{2} \mathrm{vpyRe}(\mathrm{CO})_{3}(\mathrm{tmephen})^{+}\right\}_{\mathrm{n}-180}\left\{(\mathrm{vpy})_{2} \mathrm{vpyRe}(\mathrm{CO})_{3}\left(\mathrm{NO}_{2}-\text { phen }\right)^{+}\right\}_{\mathrm{m}-20}$

$-\left\{(\mathrm{vpy})_{2} \mathrm{vpyRe}(\mathrm{CO})_{3}(\text { tmephen })^{+}\right\}_{n-150}\left\{(\mathrm{vpy})_{2} \mathrm{vpy} \operatorname{Re}(\mathrm{CO})_{3}\left(\mathrm{NO}_{2}-\mathrm{phen}\right)^{+}\right\}_{\mathrm{m} \sim 50}$

$-\left\{(\text { vpy })_{2} \text { vpyRe }(\mathrm{CO})_{3}(\text { tmephen })^{+}\right)_{\mathrm{n} \sim 100}\left\{(\mathrm{vpy})_{2} \mathrm{vpyRe}(\mathrm{CO})_{3}\left(\mathrm{NO}_{2}-\text { phen }\right)^{+}\right\}_{\mathrm{m} \sim 100}$ 


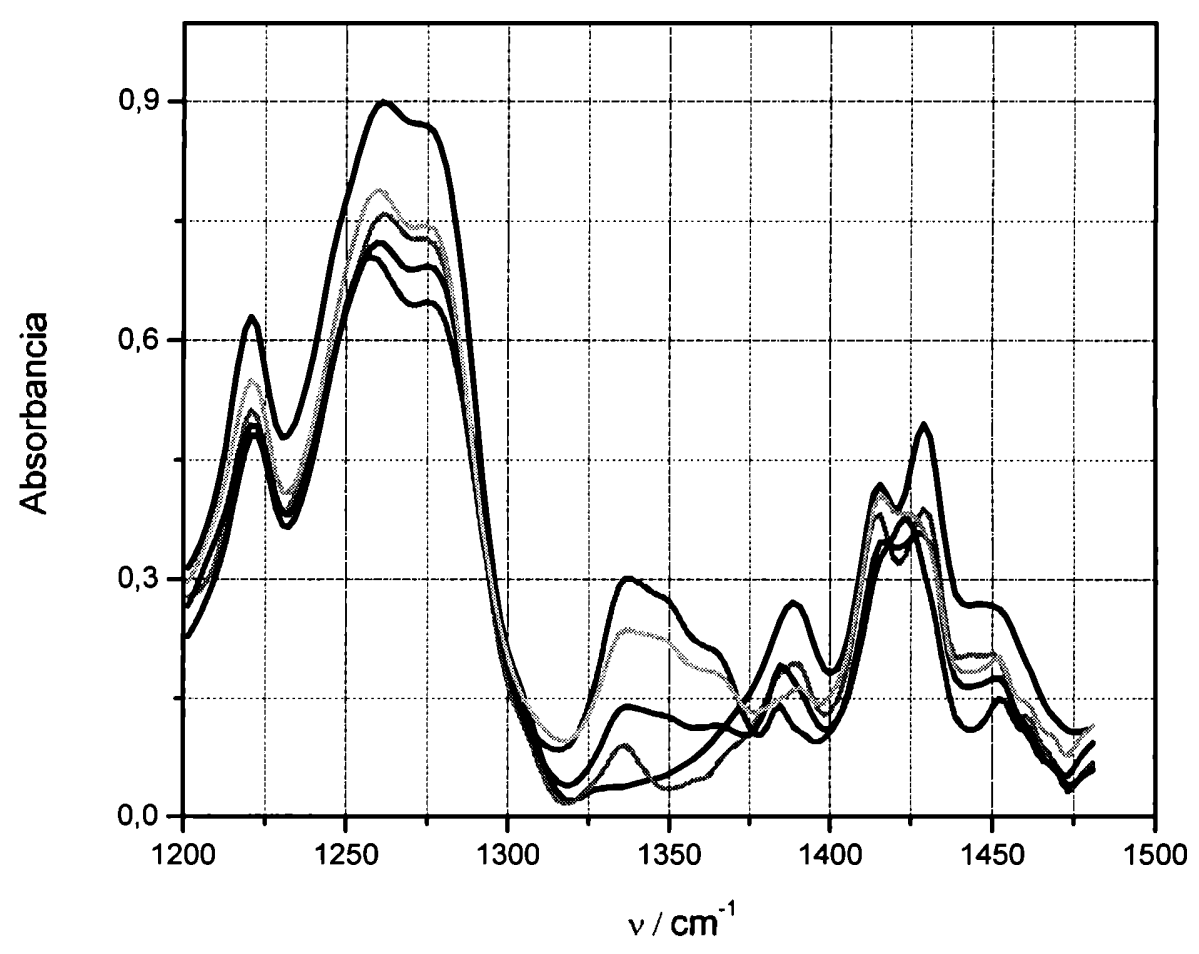

Figura 5: Espectro infrarrojo (IR) realizados en pellets de $\mathrm{KBr}$ mostrando los cambios en la región de $1300-1350 \mathrm{~cm}^{-1}$. $-\left\{(\text { vpy })_{2} \text { vpyRe(CO) }\right)_{3}$ (tmephen $\left.)^{+}\right\}_{n-200}$

$-\left\{(\text { vpy })_{2} \operatorname{vpyRe}(\mathrm{CO})_{3}\left(\mathrm{NO}_{2}-\text { phen }\right)^{+}\right\}_{n-200}$

..... $\left\{(\mathrm{vpy})_{2} \mathrm{vpyRe}(\mathrm{CO})_{3}(\text { tmephen })^{+}\right\}_{\mathrm{n} \sim 180}\left\{(\mathrm{vpy})_{2} \mathrm{vpyRe}(\mathrm{CO})_{3}\left(\mathrm{NO}_{2}-\text { phen }\right)^{+}\right\}_{\mathrm{m}-20}$

$-\left\{(\mathrm{vpy})_{2} \mathrm{vpyRe}(\mathrm{CO})_{3}(\text { tmephen })^{+}\right\}_{\mathrm{n} \sim 150}\left\{(\mathrm{vpy})_{2} \mathrm{vpyRe}(\mathrm{CO})_{3}\left(\mathrm{NO}_{2}-\mathrm{phen}\right)^{+}\right\}_{\mathrm{m} \sim 50}$ $\left\{(\mathrm{vpy})_{2} \operatorname{vpyRe}(\mathrm{CO})_{3}(\text { tmephen })^{+}\right)_{\mathrm{n} \sim 100}\left\{(\mathrm{vpy})_{2} \operatorname{vpyRe}(\mathrm{CO})_{3}\left(\mathrm{NO}_{2}-\text { phen }\right)^{+}\right\}_{\mathrm{m} \sim 100}$ 


\section{Discución y conclusiones}

\section{i. Análisis elemental}

Del análisis elemental, es posible concluir que en la síntesis de los cinco polímeros la derivatización ha ocurrido en aproximademente, 200 piridinas de las 600 que se encuentran en el esqueleto polimérico, como era de esperar de acuerdo a las cantidades molares de cada reactivo puestas a reaccionar.

\section{ii. Espectroscopia de absorción}

En el espectro de absorción se pueden apreciar las bandas correspondientes a la transición transferencia de carga metal-ligando (bandas hacia el rojo, transiciones $\mathrm{d}_{\pi \rightarrow \pi^{*}}$ ) e intraligando (bandas hacia el UV, transiciones $\pi \rightarrow \pi^{*}$ ). Ver tabla 2.

Al observar las bandas correspondientes a la TCML, es claro que el estado de $\mathrm{TCML}_{\mathrm{Re} \rightarrow \text { tmphen }}$ se genera a mayor energía que el estado excitado de $\mathrm{TCML}_{\mathrm{Re} \rightarrow \mathrm{NO2} \text {-phen }}$. Esto es debido a que el grupo $\mathrm{NO}_{2}$ es aceptor de electrones con lo cual atrae los electrones de la fenantrolina. Este hecho hace que la transferencia de carga desde el $\operatorname{Re}^{\mathrm{I}}$ hacia la nitro-1,10-fenantrolina ocurra a menor energía que la transferencia de carga desde el $\operatorname{Re}^{\mathrm{I}}$ a la 1,10-fenatrolina.

En el caso del cromóforo $-\operatorname{Re}(\mathrm{CO})_{3}$ tmephen $^{+}$, los grupos metilo son inyectores de electrones. Por este motivo la transferencia de carga desde el $\operatorname{Re}^{\mathrm{l}}$ hacia la tetrametil1,10-fenantrolina es menos favorecida que la trasferencia de carga desde el $\operatorname{Re}^{\mathrm{I}}$ a la 1,10-fenantrolina y ocurre a mayor energía. 


\section{iii Morfología de los Polímeros}

La morfología de los polímeros difiere cuando los films son obtenidos a partir de soluciones en acetonitrilo o diclorometano.

Cuando el solvente es acetonitrilo, figura 2 , en el polímero $\left\{(\mathrm{vpy})_{2} \mathrm{vpyRe}(\mathrm{CO})_{3}\left(\mathrm{NO}_{2} \text {-phen }\right)^{+}\right\}_{\mathrm{n} \sim 200}$ los complejos de $\mathrm{Re}^{\mathrm{I}}$ se observan como agregados desde nanodominios aislados a lo largo del esqueleto del polímero. Las dimensiones de los agregados varían entre 80 y $160 \mathrm{~nm}$ y son, principalmente, de forma esférica. Sin embargo el polímero $\left\{(\mathrm{vpy})_{2} \mathrm{vpyRe}(\mathrm{CO})_{3}(\mathrm{tmephen})^{+}\right\}_{n \sim 200}$, no forma agregados de nanodominios y sólo pueden observarse pequeñas esferas con un diámetro entre 5 y $30 \mathrm{~nm}$. En lo polímeros mixtos, las imágenes sugieren la formación de agregados cuyo tamaño se incrementa al pasar de $\left\{(\mathrm{vpy})_{2} \mathrm{vpyRe}(\mathrm{CO})_{3}(\text { tmephen })^{+}\right\}_{\mathrm{n} \sim 180}\left\{(\mathrm{vpy})_{2} \mathrm{vpyRe}(\mathrm{CO})_{3}\left(\mathrm{NO}_{2} \text {-phen }\right)^{+}\right\}_{\mathrm{m} \sim 20}$ $\left\{(\mathrm{vpy})_{2} \mathrm{vpyRe}(\mathrm{CO})_{3}(\text { tmephen })^{+}\right\}_{\mathrm{n} \sim 100}\left\{(\mathrm{vpy})_{2} \mathrm{vpyRe}(\mathrm{CO})_{3}\left(\mathrm{NO}_{2}-\text { phen }\right)^{+}\right\}_{\mathrm{m} \sim 100}$.

Las dimensiones de los nanodominios son considerablemente mayores que el tamaño correspondiente a la cadena de polímero, por lo cual es posible inferir que dichos nanodominios contienen mas de una cadena de polímero.

La situación es muy diferente en diclorometano, figura 3. En los polímeros $\left\{(\text { vpy })_{2} \operatorname{vpyRe}(\mathrm{CO})_{3}(\text { tmephen })^{+}\right\}_{\mathrm{n}-200}$,

$\left\{(\mathrm{vpy})_{2} \mathrm{vpyRe}(\mathrm{CO})_{3}(\text { tmephen })^{+}\right\}_{\mathrm{n} \sim 180}\left\{(\mathrm{vpy})_{2} \mathrm{vpyRe}(\mathrm{CO})_{3}\left(\mathrm{NO}_{2}-\text { phen }\right)^{+}\right\}_{\mathrm{m} \sim 20} \quad \mathrm{y}$

$\left\{(\mathrm{vpy})_{2} \mathrm{vpyRe}(\mathrm{CO})_{3}(\text { tmephen })^{+}\right\}_{\mathrm{n} \sim 150}\left\{(\mathrm{vpy})_{2} \mathrm{vpyRe}(\mathrm{CO})_{3}\left(\mathrm{NO}_{2}-\text { phen }\right)^{+}\right\}_{\mathrm{m} \sim 50}, \quad$ puede observarse la formación de vesículas, que son evidenciadas por la alta transmición en el centro de los agregados, con respecto a los alrededores. El tamaño de las vesículas es polidisperso, y sus diámetros varían entre $140 \mathrm{~nm}$ y $1.4 \mu \mathrm{m}$. 
El polimero $\left\{(\mathrm{vpy})_{2} \mathrm{vpyRe}(\mathrm{CO})_{3}\left(\mathrm{NO}_{2} \text {-phen }\right)^{+}\right\}_{\mathrm{n}-200}$ forma estructuras en rama tubular como entretejiendo una red.

Las morfologías observadas en el polímero $\left\{(\text { vpy })_{2} \mathrm{vpyRe}(\mathrm{CO})_{3}(\text { tmephen })^{+}\right\}_{\mathrm{n} \sim 100}\left\{(\mathrm{vpy})_{2} \mathrm{vpyRe}(\mathrm{CO})_{3}\left(\mathrm{NO}_{2}-\text { phen }\right)^{+}\right\}_{\mathrm{m}-100}$ son intermedias entre forma de vesículas y túbulos.

En trabajos anteriores se han obsevado agregados miscelares en los polímeros $\left.\left\{\left(\text { vpy- }\left[\operatorname{Re}(\mathrm{CO})_{3}\left(2,2^{\prime}-\text { bpy }\right)\right]\right)_{\mathrm{m}}\left(\mathrm{vpy}-\left[\operatorname{Re}(\mathrm{CO})_{3}(\text { phen })\right]\right)_{\mathrm{n}}(\mathrm{vpy})_{\mathrm{p}}\right\}-\left(\mathrm{CF}_{3} \mathrm{SO}_{3}\right)_{\mathrm{m}+\mathrm{n}}\right\}$, en films obtenidos a partir de soluciones en acetonitrilo. ${ }^{5-6} \mathrm{~A}$ partir de estas observaciones se ha llegado a concluir lo siguiente: poli4-vinilpiridina (vpy) $)_{600}$ es bastante insoluble en acetonitrilo, pero este solvente es bueno para los polímeros Re-(vpy) $)_{60}$. Es posible imaginar que el interior de la miscela en acetonitrilo está formado principalmente por piridinas libres de $\operatorname{Re}-(\mathrm{vpy})_{600}$ y la parte externa estará constituida principalmente por los pendientes de $\operatorname{Re}^{\mathrm{I}}$. La situación es inversa en diclorometano dado que este es un buen solvente para $(v p y)_{600}$. Sin embargo los polímeros $\left\{\left(v p y-\left[\operatorname{Re}(C O)_{3}\left(2,2^{\prime}-\right.\right.\right.\right.$ bpy) $\left.\left.])_{\mathrm{m}}\left(\mathrm{vpy}-\left[\operatorname{Re}(\mathrm{CO})_{3}(\mathrm{phen})\right]\right)_{\mathrm{n}}(\mathrm{vpy})_{\mathrm{p}}\right\}-\left(\mathrm{CF}_{3} \mathrm{SO}_{3}\right)_{\mathrm{m}+\mathrm{n}}\right\}$ no son solubles en este solvente, mientras que los polímeros sintetizados para este trabajo de tesis son considerablemente menos solubles en diclorometano que en acetonitrilo. En la vesículas sin embargo, un pool de moléculas de diclorometano están solvatando las piridinas libres del polímero en la región de afuera de la vesícula, mientras que los pendientes de $\mathrm{Re}^{\mathrm{I}}$ estarán principalmente en el interior de la membrana de la vesícula

\section{iv. FT-IR}

Tanto en la tabla 3 como en la figura 4 puede observarse que hay un corrimiento de $v(\mathrm{CO})$ a números de onda mas altos cuando el contenido de los grupos 
$-\operatorname{Re}(\mathrm{CO})_{3}\left(\mathrm{NO}_{2} \text {-phen }\right)^{+}$se incrementa relativo al número de pendientes

$-\operatorname{Re}(\mathrm{CO})_{3}(\text { tmphen })^{+}$desde el polímero

$\left\{(\mathrm{vpy})_{2} \mathrm{vpyRe}(\mathrm{CO})_{3}(\text { tmephen })^{+}\right\}_{\mathrm{n} \sim 180}\left\{(\mathrm{vpy})_{2} \mathrm{vpyRe}(\mathrm{CO})_{3}\left(\mathrm{NO}_{2}-\text { phen }\right)^{+}\right\}_{\mathrm{m} \sim 20}$ al

$\left\{(\mathrm{vpy})_{2} \mathrm{vpyRe}(\mathrm{CO})_{3}(\text { tmephen })^{+}\right\}_{\mathrm{n} \sim 100}\left\{(\mathrm{vpy})_{2} \mathrm{vpyRe}(\mathrm{CO})_{3}\left(\mathrm{NO}_{2}-\mathrm{phen}\right)^{+}\right\}_{\mathrm{m} \sim 100}$.

Además en la región entre $1300-1350 \mathrm{~cm}^{-1}$, donde el ligando tmephen no tiene absorción significante, el ligando $\mathrm{NO}_{2}$-phen presenta una absorción característica centrada en $1330 \mathrm{~cm}^{-1}$. En los polímeros mixtos se incrementa la absorción a $1330 \mathrm{~cm}^{-1}$ cuando se incrementa la cantidad de pendientes $\operatorname{Re}(\mathrm{CO})_{3}\left(\mathrm{NO}_{2} \text {-phen }\right)^{+}$relativo a los pendientes $\operatorname{Re}(\mathrm{CO})_{3}(\text { tmphen })^{+}$cuando se pasa de $\left\{(\mathrm{vpy})_{2} \mathrm{vpyRe}(\mathrm{CO})_{3}(\text { tmephen })^{+}\right\}_{\mathrm{n} \sim 180}\left\{(\mathrm{vpy})_{2} \mathrm{vpyRe}(\mathrm{CO})_{3}\left(\mathrm{NO}_{2}-\text { phen }\right)^{+}\right\}_{\mathrm{m} \sim 20}$ $\left\{(\mathrm{vpy})_{2} \mathrm{vpyRe}(\mathrm{CO})_{3}(\text { tmephen })^{+}\right\}_{\mathrm{n} \sim 100}\left\{(\mathrm{vpy})_{2} \mathrm{vpyRe}(\mathrm{CO})_{3}\left(\mathrm{NO}_{2}-\text { phen }\right)^{+}\right\}_{\mathrm{m} \sim 100}$. 


\section{Referencias}

1. E. Wolcan and G. Ferraudi, Jof Phys. Chem. A. 2000, 104, 9281.

2. E. Wolcan and M. R. Féliz, Photochem. Photobiol. Sci. 2003, 2, 412.

3. M. S. Wrighton and D. L. Morse, J. Am. Chem. Soc. 1974, 100, 5790.

4. P. J. Giordano, S. M. Fredericks, M.S. Wrighton, and D. L. Morse, J. Am. Chem. Soc. 1978, 100, 2257.

5. E. Wolcan; J. L. Alessandrini; and M. R. Féliz, J. Phys. Chem. B. 2005, 109, 22890.

6. E. Wolcan ; M. R. Féliz., J. L. Alessandrini; and G. Ferraudi. Inorg. Chem. 2006, 45, 6666 . 
Capítulo II

Transferencia de energía en polímeros $\mathrm{Re}^{\mathrm{I}}$ 


\section{Capítulo II}

\section{Introducción}

La fotogeneración de los estados excitados de TCML en un polímero posibilita el estudio de procesos de transferencia de energía intramolecular suponiendo que "Donor" y “Aceptor" penden de un mismo esqueleto polimérico.

La transferencia de energía resonante (RET) es un procesos fotofísico por el cual una molécula, donor, transfiere su energía de excitación a una molécula aceptor con la consecuente disminución del tiempo de vida del donor.

Si el Donor es una molécula fluorescente, el proceso es referido como transferencia de energía resonante fluorescente, FRET. La teoría de Förster ${ }^{1}$ por ejemplo, es usada para el estudio de interfase en mezclas de polímeros, separación de fases y dinámica conformacional de polímeros. ${ }^{2-5}$

En ciencias biológicas esta teoría es utilizada para el diseño de sistemas supramoleculares que pueden ser usados como captadores de luz en fotosíntesis artificial, como en los sistemas de captación de luz de plantas y bacterias fotosintéticas que involucran la transferencia unidireccional de energía de radiación absorbida por el centro de reacción, vía un mecanismo multipasos de FRET. Además FRET es comúnmente usado en la química de sensores. $^{6-10}$

En este capítulo se muestran los resutados experimentales del estudio de emisión estacionaria, y de la emisión y la absorción resuelta en el tiempo de los polímeros sintetizados. $\left\{(\text { vpy })_{2} \text { vpyRe}(\mathrm{CO})_{3}(\text { tmephen })^{+}\right\}_{\mathrm{n} \sim 200},\left\{(\mathrm{vpy})_{2} \mathrm{vpyRe}(\mathrm{CO})_{3}\left(\mathrm{NO}_{2} \text { phen }\right)^{+}\right\}_{\mathrm{n} \sim 200}$, 
$\left\{(\mathrm{vpy})_{2} \mathrm{vpyRe}(\mathrm{CO})_{3}(\mathrm{tmephen})^{+}\right\}_{\mathrm{n} \sim 180}\left\{(\mathrm{vpy})_{2} \mathrm{vpyRe}(\mathrm{CO})_{3}\left(\mathrm{NO}_{2}-\mathrm{phen}\right)^{+}\right\}_{\mathrm{m} \sim 20}$,

$\left\{(\mathrm{vpy})_{2} \mathrm{vpyRe}(\mathrm{CO})_{3}(\text { tmephen })^{+}\right\}_{\mathrm{n} \sim 150}\left\{(\mathrm{vpy})_{2} \mathrm{vpyRe}(\mathrm{CO})_{3}\left(\mathrm{NO}_{2}-\text { phen }\right)^{+}\right\}_{\mathrm{m} \sim 50} \mathrm{y}$

$\left\{(\text { vpy })_{2} \operatorname{vpyRe}(\mathrm{CO})_{3}(\text { tmephen })^{+}\right)_{\mathrm{n} \sim 100}\left\{(\text { vpy })_{2} \operatorname{vpyRe}(\mathrm{CO})_{3}\left(\mathrm{NO}_{2}-\text { phen }\right)^{+}\right\}_{\mathrm{m} \sim 100}$

Además de la información que puede obtenerse de estos resultados, como rendimientos cuánticos de emisión y tiempos de vida de los transientes para cada uno de los polímeros, ha quedado en evidencia la desactivación de la luminiscencia del estado excitado de $\mathrm{TCML}_{\mathrm{Re} \rightarrow \text { tmphen }}$ en los polímeros mixtos, por la presencia del cromóforo $-\mathrm{Re}(\mathrm{CO})_{3}\left(5 \mathrm{NO}_{2}\right.$ phen $)^{+}$.

De los resultados de láser flash fotólisis fue posible concluir que esta desactivación se realiza por un mecanismo de transferencia de energía, por lo tanto en primer lugar se hará un desarrollo teórico de los conceptos de transferencia de energía en general, para luego desarrollar este mecanismo en polímeros, a continuación se mostrarán los resultados experimentales para terminar el capítulo con la discusión y conclusiones. 


\section{Transferencia de energía. Conceptos teóricos}

\section{i. Introducción}

Si se considera el sistema de moléculas * $\mathrm{R}$ y $\mathrm{A}$, donde ${ }^{*} \mathrm{R}$ es una especie en estado excitado y A es una especie en estado fundamental; y se plantean las diferentes interacciones orbitales entre ellas, pueden obtenerse diferentes resultados

$$
\begin{array}{ll}
{ }^{*} \mathrm{R}+\mathrm{A} \rightarrow \mathrm{R}+{ }^{*} \mathrm{~A} & \text { Transferencia de energia (* } \mathrm{R} \text { donor) } \\
{ }^{*} \mathrm{R}(\mathrm{eD})+\mathrm{A}(\mathrm{eA}) \rightarrow \mathrm{R}^{+} / \mathrm{A}^{-} & \text {Transferencia de electrones (*R donor) } \\
{ }^{*} \mathrm{R}(\mathrm{eA})+\mathrm{A}(\mathrm{eD}) \rightarrow \mathrm{R}^{-} / \mathrm{A}^{+} & \text {Transferencia de electrones (*R aceptor) }
\end{array}
$$

El primer proceso describe una transferencia de energía, mientras que el segundo y tercer proceso representan una transferencia de electrones.

El proceso de transferencia de energía usualmente ocurre a una velocidad alta si la energía del estado excitado $E\left({ }^{*} R\right)$ es mayor que la energía del estado excitado $E\left({ }^{*} A\right)$, en tal caso la energía del estado * $\mathrm{R}$ es suficiente para generar el estado * A. La velocidad del proceso de transferencia de electrones es determinada no sólo por la energía del estado excitado sino también por la termodinámica del proceso redox.

Para el caso de transferencia de energía, se llamará *D al estado electrónicamente excitado por ser siempre el donor y A al estado fundamental aceptor.

Además de la transferencia de energía, existen otras vías de desactivación del estado *D, y se muestran a continuación: 


$$
\begin{aligned}
& * \mathrm{D} \quad \stackrel{k_{\mathrm{D}}}{\rightarrow} \mathrm{D}(+ \text { hv ó } \Delta) \\
& k_{\mathrm{ET}} \\
& { }^{*} \mathrm{D}+\mathrm{A} \rightarrow \mathrm{D}+{ }^{*} \mathrm{~A} \\
& k_{\mathrm{nr}} \\
& * \mathrm{D}+\mathrm{A} \rightarrow \mathrm{D}+\mathrm{A} \\
& k_{\text {rxn }} \\
& { }^{*} \mathrm{D}+\mathrm{A} \rightarrow \text { Cambio químico }
\end{aligned}
$$

donde,

$k_{\mathrm{D}}$ : Constante de velocidad de desactivación del estado excitado ${ }^{*} \mathrm{D}$ que no involucran al aceptor A. Emisión de luz y/o calor.

$k_{\mathrm{ET}}$ : Constante de velocidad del proceso de transferencia de energía.

$k_{\mathrm{nr}}$ : Constante de velocidad de desactivación no radiativa de ${ }^{*} \mathrm{D}$ que involucra al aceptor A. $k_{\text {rxn }}$ : Constante de velocidad de reacción química entre $*$ D y A.

Suponiendo que es posible determinar una constante de velocidad experimental para la desactivación, ó quenching total ${ }^{*} \mathrm{D}$ por la presencia de $\mathrm{A}, \mathrm{kq}$ involucra todos los proceso descriptos como se muestra en la ecuación:

$$
k_{\mathrm{q}}=k_{\mathrm{ET}}+k_{\mathrm{nr}}+k_{\mathrm{rxn}}
$$

La eficiencia de la transferencia de energía $\phi_{\mathrm{ET}}$ esta dada por:

$$
\phi_{E T}=\frac{k_{E T}[A]}{k_{D}+k_{q}[A]}=\frac{k_{E T}[A]}{k_{D}+\left(k_{E T}+k_{n r}+k_{n r x}\right)[A]}
$$

$\phi_{\mathrm{ET}}$ corresponde a la fracción de moléculas de ${ }^{*} \mathrm{D}$ que decaen vía transferencia de energía.

El rendimiento cuántico de la transferencia de energía $\Phi_{\mathrm{ET}}$, considera además el rendimiento cuántico con el cual se generó * D: 


$$
\Phi_{E T}=\Phi_{*_{D}} \cdot \phi_{E T}
$$

donde $\Phi_{*_{D}}$ : Rendimiento cuántico de formación de ${ }^{*} \mathrm{D}$.

Existen distintos mecanismos de transferencia de energía que dependen de las características del sistema.

\section{ii. Mecanismos de Transferencia de energía}

\section{a. Mecanismo radiativo o "Trivial"}

En este mecanismo el Donor y el Aceptor actúan independientemente, es decir no hay interacción que "dispare" la transferencia.

*D emite un fotón que puede ser capturado por A resultando una trasferencia de energía entre ambos. El aceptor intercepta el fotón una vez que ha sido emitido con lo cual no altera las características de emisión o tiempo de vida del estado excitado ${ }^{*} \mathrm{D}$.

Este mecanismo no requiere un encuentro físico entre las moléculas participantes. La transferencia de energía radiativa, trivial, ocurre en dos pasos cuya secuencia se muestran en las siguientes ecuaciones:

$$
\begin{aligned}
& { }^{* D} \quad \rightarrow \mathrm{D}+\mathrm{hv} \\
& \mathrm{hv}+\mathrm{A} \rightarrow{ }^{* A}
\end{aligned}
$$

El mecanismo trivial requiere que los fotones emitidos por ${ }^{*} \mathrm{D}$ sean absorbidos por $\mathrm{A}$, en otras palabras, el espectro de emisión de ${ }^{*} \mathrm{D}$ debe solapar con el espectro de absorción de $\mathrm{A}$. 
La velocidad o probabilidad por unidad de tiempo de transferencia de energía entre ${ }^{*} \mathrm{D}$ y $\mathrm{A}$ dependerá de

a- El rendimiento cuántico de emisión de ${ }^{*} \mathrm{D}$

b- El número de moléculas de $\mathrm{A}$ en el camino de los fotones emitidos por *D

c- Espectro de absorción de A

d- El solapamiento del espectro de emisión de * D y el espectro de absorción de A

La transferencia de energía trivial será favorecida cuando cada uno de estos parámetros es maximizado.

\section{b. Mecanismo no-radiativo}

La transferencia de energía puede tener lugar a través de dos mecanismos no-radiativos entre *D y A que serán descriptos como

b.1. Solapamiento orbital, intercambio electrónico o mecanismo de Dexter

b.2. Dipolo-Dipolo, Coulómbico o mecanismo de Förster

Los términos dipolo-dipolo, Coulómbico o Förster describen la transferencia de energía que corresponde a la interacción de dipolos, y el término solapamiento orbital intercambio electrónico o Dexter son utilizados para describir la transferencia de energía correspondiente a la interacción vía intercambio de electrones, figura 1. 

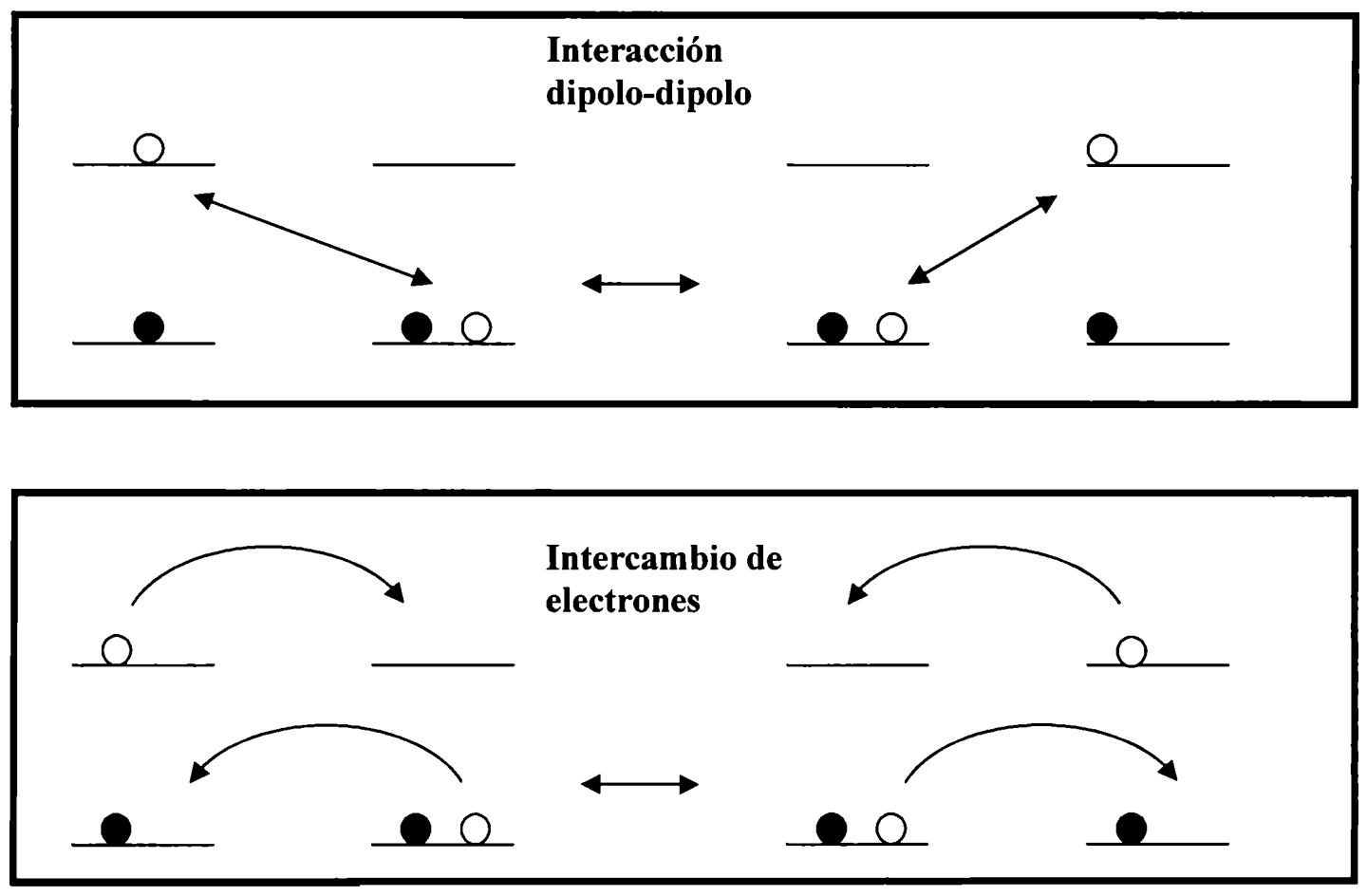

Figura 1: Comparación de transferencia de energía por el mecanismo dipolo-dipolo e intercambio electrónico

La diferencia entre los dos mecanismos es que cuando se dispara la transferencia de energía, en el mecanismo dipolo-dipolo el electrón no cambia de molécula, pero las dos transiciones $\left({ }^{*} \mathrm{D} \rightarrow \mathrm{D} \quad\right.$ y $\left.\quad \mathrm{A} \rightarrow{ }^{*} \mathrm{~A}\right)$ ocurren simultáneamente. La excitación de $\mathrm{A}$ es un mecanismo análogo al dado por absorción de luz, sólo que en este caso el campo electromagnético lo provee la oscilación del dipolo.

Se puede relacionar la constante de transferencia de energía $k_{\mathrm{ET}}$, con los correspondientes Hamiltonianos para las interacciones coulómbica y de intercambio de electrones por la ecuación:

$$
k_{E T}=\underbrace{\alpha\left\langle\varphi\left(D^{*}\right) \varphi(A) H_{e} \varphi(D)_{\kappa}\left(A^{*}\right)\right\rangle}_{\text {Intercambio electrónico }}+\underbrace{\beta\left\langle\varphi\left(D^{*}\right) \varphi(A) H_{c} \varphi(D) \varphi\left(A^{*}\right)\right\rangle}_{\text {Interacción dipolo-dipolo }}
$$


Aunque a continuación se tratarán ambos procesos por separado.

\section{b.1. Proceso de intercambio electrónico: Transferencia de energía por colisión}

Si se consideran los orbitales como esferas, es posible estimar la relación del solapamiento orbital con la distancia entre donor y aceptor y también la constante para la transferencia de energía por intercambio electrónico. Por lo tanto para dos orbitales esféricos separados, centrados en ${ }^{*} \mathrm{D}$ y $\mathrm{A}$, se espera que el solapamiento orbital decrezca exponencialmente con el incremento en la separación entre ${ }^{*} \mathrm{D}$ y A. Debido a que la velocidad de transferencia de energía por intercambio electrónico está directamente relacionada con el solapamiento orbital de ${ }^{*} \mathrm{D}$ y $\mathrm{A}$, es de esperar que la constante de velocidad del proceso disminuya como una función exponencial de la separación entre *D y A. Además la constante de velocidad del proceso estará relacionada con $\mathbf{J}$, la integral del solapamiento espectral, la cual es una medida de los estados que alcanzan la condición de resonancia.

Dexter $^{11}$ propuso para la transferencia de energía por intercambio electrónico que la constante de velocidad de la transferencia de energía está dada por:

$$
\left.k_{E T} \text { (int ercambio }\right)=K J \exp \left(-2 r_{D A} / L\right)
$$

Donde, $\mathrm{K}$ está relacionado con la interacción orbital específica entre ${ }^{*} \mathrm{D}$ y $\mathrm{A}$ dependiendo de la orientación; $\mathbf{J}$ es la integral de solapamiento espectral normalizada, donde el término normalizada se refiere a que, tanto el área bajo la curva de intensidad de emisión, $\mathrm{I}_{\mathrm{D}}$, como el coeficiente de extinción $\varepsilon_{\mathrm{A}}$ han sido ajustados a la unidad sobre la escala de 
número de onda. $\mathrm{r}_{\mathrm{DA}}$ es la separación donor-aceptor relativa al radio de Van der Waals, L. Por definición $\mathrm{r}_{\mathrm{DA}}$ corresponde a la separación entre bordes, mientras que la separación entre centros será expresada como $\mathrm{R}_{\mathrm{DA}}$.

El proceso de transferencia de electrones será desarrollado en profundidad en la Parte II Capítulo IV.

\section{b.2. Visualización de la transferencia de energía por interacción dipolo-dipolo. Teoría de Förster}

La interacción dipolo-dipolo representa una acción a distancia en la cual los electrones que inicialmente están en *D luego están en $\mathrm{D}$ y los que estaban en $\mathrm{A}$ también estarán en *A, es decir la interacción ocurre vía la interacción del campo electromagnético del dipolo oscilante en *D con los electrones de A.

La interacción dipolo-dipolo representa una interacción coulómbica clásica entre partículas cargadas. El campo eléctrico cercano a una molécula electrónicamente excitada se supone igual al campo generado por un dipolo eléctrico oscilante cuya frecuencia de oscilación es $v$ y el dipolo instantáneo es $\mu$. Si $\left[\mu_{0}\right]$ es el máximo valor del dipolo inducido para la determinación del dipolo instantáneo se aplica la siguiente ecuación:

$$
\mu=\mu_{0} \cos (2 \pi v t)
$$

donde $t$ es el tiempo. 
Clásicamente para el estado fundamental de $\mathrm{A}$, se supone que los electrones no poseen ninguna oscilación. El resultado de la oscilación del dipolo de ${ }^{*} \mathrm{D}$ causará la excitación de sistemas electrónicos de moléculas cercanas si se cumplen ciertas condiciones de resonancia.

Para la absorción de luz de A, la condición de resonancia está dada por la ecuación:

$$
\Delta \mathrm{E}\left(\mathrm{A} \rightarrow{ }^{*} \mathrm{~A}\right)=\mathrm{hv}
$$

En este caso el acoplamiento es el resultado de la interacción del campo electromagnético y los electrones de A.

Para la transferencia de energía la condición de resonancia está dada por la siguiente ecuación:

$$
\Delta \mathrm{E}\left({ }^{*} \mathrm{D} \rightarrow \mathrm{D}\right)=\Delta \mathrm{E}\left(\mathrm{A} \rightarrow{ }^{*} \mathrm{~A}\right)
$$

y el acoplamiento está dado por la interacción dipolo-dipolo (de frecuencia correcta) de la oscilación del dipolo de ${ }^{*} \mathrm{D}$ con los electrones de $\mathrm{A}$.

Es decir que el cambio de energía del proceso global debe ser igual a cero. La energía del estado excitado del donor y el aceptor son por lo general diferentes por lo tanto serán requeridos otros modos para la conservación de la energía, por ejemplo los modos vibracionales y rotacionales del donor y aceptor, pueden recibir energía. En solución estas especies usualmente alcanzan el equilibrio térmico rápidamente por interacción con el solvente. 


\section{Aspectos cuantitativos de la teoría de Förster}

¿Cuáles son los factores que influyen en la interacción responsable de la transferencia de energía desde $* D$ hacia $A$ ?

De acuerdo con la teoría clásica la energía de interacción electrostática $\mathrm{E}$, entre dos dipolos eléctricos está directamente relacionada con la magnitud de dos dipolos interactuantes $\left(\mu_{D}\right.$ y $\left.\mu_{A}\right)$ y la distancia entre ellos $\left(R_{D A}\right)$ como muestra la ecuación

$$
E \text { (dipolo-dipolo }) \alpha \frac{\mu_{D} \mu_{A}}{R_{D A}^{3}}
$$

Förster relaciona $\mu_{\mathrm{D}}$ y $\mu_{\mathrm{A}}$ con la fuerza del oscilador $\mathrm{f}$, para la transición radiativa

${ }^{*} \mathrm{D} \leftrightarrow \mathrm{D}$ y $\mathrm{A} \leftrightarrow{ }^{*} \mathrm{~A}$. La energía de interacción dipolo-dipolo queda expresada entonces en términos de $f_{D} \mathrm{y}_{\mathrm{A}}$, la medida de la fuerza del oscilador para la transición radiativa de $\mathrm{D}$ y $\mathrm{A}$, la cual incluye factores electrónicos, vibracionales y de spin.

Förster muestra que $k_{\mathrm{ET}}$ puede ser relacionada con $\mathrm{E}^{2}$ cuantitativamente por medio de la ecuación:

$$
k_{E T}(\text { dipolo-dipolo }) \alpha E^{2} \approx\left(\frac{\mu_{D} \mu_{A}}{R^{3}}\right)^{2}=\frac{\mu_{D}^{2} \mu_{A}^{2}}{R_{D A}^{6}}
$$

En esta ecuación la velocidad de transferencia de energía disminuye con el aumento en la separación $\mathrm{R}_{\mathrm{DA}}$ entre $* \mathrm{D}$ y $\mathrm{A}$. 
Förster relaciona las cantidades teóricas de la fuerza del oscilador y transición de dipolos con cantidades directamente medibles. Las relaciones entre los momentos de transición y cantidades experimentales son:

$$
\begin{aligned}
& \mu_{D}^{2}\left(D^{*} \leftrightarrow D\right) \rightarrow \int \varepsilon_{A} o k_{D}^{0} \\
& \mu_{A}^{2}\left(A^{*} \leftrightarrow A\right) \rightarrow \int \varepsilon_{A} o k_{A}^{0}
\end{aligned}
$$

Donde, $\int \varepsilon$ es la integral del coeficiente de extinción de la banda de absorción y $\mathrm{k}^{0}$ es la constante de velocidad radiativa.

Considerando un proceso de transferencia de energía donde $\mathrm{D}^{*} \leftrightarrow \mathrm{D}$ y $\mathrm{A} \leftrightarrow \mathrm{A}^{*}$, se puede reemplazar el término del cuadrado de la transición electrónica por términos experimentales $k_{D}^{0}$ y $\int \varepsilon_{A}$

$$
k_{E T}(\text { Coulómbica }) \rightarrow \frac{k_{D}^{0} \int \varepsilon}{R_{D A}^{0}}
$$

Finalmente, se reorganizan los requerimientos de solapamiento orbital y se considera el solapamiento de la emisión de ${ }^{*} \mathrm{D}$ con la absorción de A

$$
k_{E T(\text { coulómbico })}=\alpha \frac{\kappa^{2} k_{D}^{0}}{R_{A D}^{0}} J\left(\varepsilon_{A}\right)
$$


El término $\alpha$ es una constante determinada por condiciones experimentales como concentración e índice de refracción del solvente. El término $\kappa^{2}$ es un factor de interacción entre dos dipolos oscilantes y depende de la orientación mutua de dipolos en el espacio. Para una distribución aleatoria de dipolos interaccionantes, $\kappa^{2}$ es una constante: $2 / 3$. El término $\int\left(\varepsilon_{A}\right)$ es similar a la integral de solapamiento con el coeficiente de extinción del aceptor $(\varepsilon)$ incluido en la integración.

\section{Relación entre la eficiencia de transferencia de energía y separación donor-aceptor}

Conceptualmente, la eficiencia de la transferencia de energía es la fracción de moléculas de ${ }^{*} \mathrm{D}$ que transfieren su energía al aceptor, A. Es conveniente definir una eficiencia para la cual la velocidad de transferencia de energía sea igual a la suma de velocidades de desactivación de ${ }^{*} \mathrm{D}$ :

$$
k_{E T}\left[D^{*}\right][A]=k_{D}\left[D^{*}\right] \quad \text { Para } \mathrm{R}_{\mathrm{AD}}=\mathrm{R}_{\mathrm{F}}
$$

ó

$$
\mathrm{k}_{\mathrm{ET}}[\mathrm{A}]=\mathrm{k}_{\mathrm{D}}=\tau_{\mathrm{D}}^{-1}
$$

$\mathrm{R}_{\mathrm{F}}$ : Radio de Förster, "separación crítica"para la cual la velocidad de transferencia de energía y la velocidad de desactivación de $* \mathrm{D}$ son iguales.

El radio crítico de Förster $R_{F}$ puede ser calculado por la siguiente ecuación:

$$
\left(\frac{R_{F}}{c m}\right)^{6}=8.79 \times 10^{-25} \frac{\kappa^{2} \phi_{D}}{n^{4}} \int \frac{f_{D}(v) \varepsilon_{A}(v) d v}{d m^{3} m o l^{-1} \mathrm{~cm}^{3} v^{4}}
$$


Donde, $\kappa^{2}$ : es un factor de orientación igual a $2 / 3$ para una distribución isotrópica angular; $n$ : es el índice de refracción del medio; $\phi_{D}$ : es el rendimiento cuántico de emisión del donor; $\varepsilon_{A}(v)$ es el coeficiente de extinción molar del aceptor para el número de onda $v$ y $f_{D}(v)$ es el espectro de emisión del donor cuando $\int f_{D}(v) d v=1$

La constante de velocidad y la eficiencia para la transferencia de energía por el mecanismo dipolo-dipolo puede relacionarse con la separación entre * D y A por medio de la siguiente expresión:

$$
k_{E T} \alpha k_{D}\left(\frac{R_{F}}{R}\right)^{6}=\frac{1}{\tau_{D}}\left(\frac{R_{F}}{R}\right)^{6}
$$

donde $\tau_{D}$ es el tiempo de vida de ${ }^{*} \mathrm{D}, \mathrm{R}$ es la separación entre centros de ${ }^{*} \mathrm{D}$ y $\mathrm{A}$. $\mathrm{R}_{\mathrm{F}}$ es la separación crítica entre ${ }^{*} \mathrm{D}$ y A para la cual la transferencia de energía y la emisión desde *D son igualmente probables.

La eficiencia a cualquier separación, será:

$$
\phi_{E T} \alpha\left(\frac{R_{F}}{R}\right)^{6}
$$




\section{iii. Comparación de la interacción dipolo-dipolo (o Förster) y la interacción por solapamiento orbital (o Dexter)}

a. La constante de velocidad de la transferencia de energía dipolo-dipolo decrece con $\mathrm{R}^{-6}$ mientras que la constante de velocidad de intercambio electrónico decrece como exp($2 \mathrm{r} / \mathrm{L})$

b. La constante de velocidad de la transferencia dipolo-dipolo depende de la fuerza del oscilador de la transición radiativa $* \mathbf{D} \rightarrow \mathbf{D}$ y $\mathbf{A} \rightarrow{ }^{*} \mathrm{~A}$, pero la constante de velocidad de transferencia por intercambio es independiente de la fuerza del oscilador de la transición ${ }^{*} \mathbf{D} \rightarrow \mathbf{D} \mathbf{y} \mathbf{A} \rightarrow{ }^{*} \mathbf{A}$

c. La eficiencia de la transferencia de energía para el mecanismo dipolo-dipolo depende principalmente de la longitud del oscilador de la transición $\mathbf{A} \rightarrow{ }^{*} \mathrm{~A}$ y está directamente relacionada con $\phi_{\mathrm{D}}$, mientras que la eficiencia de la transferencia de energía por interacción de intercambio no puede ser directamente relacionada con cantidades experimentales.

Ambas teorías, Förster y Dexter, predicen la dependencia directa de $k_{\mathrm{ET}}$ con $\mathbf{J}$, la integral de solapamiento orbital. 


\section{iv. Transferencia de energía en ausencia de difusión}

La transferencia de energía puede ocurrir en soluciones sólidas bajo condiciones en las cuales la difusión molecular es imposible, o en sistemas donde donor y aceptor no tengan la posibilidad de cambiar de posición uno con respecto al otro. En este caso es posible usar la formulación de Perrin, que supone la existencia de una esfera de quenching alrededor de *D. Las suposiciones del modelo de Perrin son:

1. Donor y aceptor no pueden sufrir desplazamiento en el espacio durante el tiempo de vida de ${ }^{*} \mathrm{D}$.

2. Existe un volumen en el espacio, "esfera de quenching" alrededor de *D con radio R. Si una molécula desactivante se encuentra dentro de la esfera de quenching entonces la eficiencia de desactivación de *D será igual a 1 .

3. Si una molécula desactivante se encuentra fuera de la esfera entonces, no desactivará a *D.

El modelo de Perrin predice la siguiente relación:

$$
\ln \left(\phi^{0} / \phi_{D}\right)=V[Q]
$$

Donde $\phi^{0}$ y $\phi_{D}$ son los rendimientos cuánticos de emisión en ausencia y en presencia de desactivante, $\mathrm{V}$ es el volumen de la esfera activa de quenching en $\mathrm{cm}^{3}, \mathrm{~N}$ es el número de Avogadro y $[Q]$ es la concentración de desactivante expresada en Molar.

La relación entre el radio de la esfera de quenching y la concentración de desactivante, aceptor, está dada por: 


$$
\mathrm{R}_{\mathrm{F}}[\AA]=6.5[\mathrm{~A}]^{-1 / 3}
$$

Donde R está expresado en $\AA$ y $[A]$ es la concentración de aceptor expresada en Molar (figura 2).

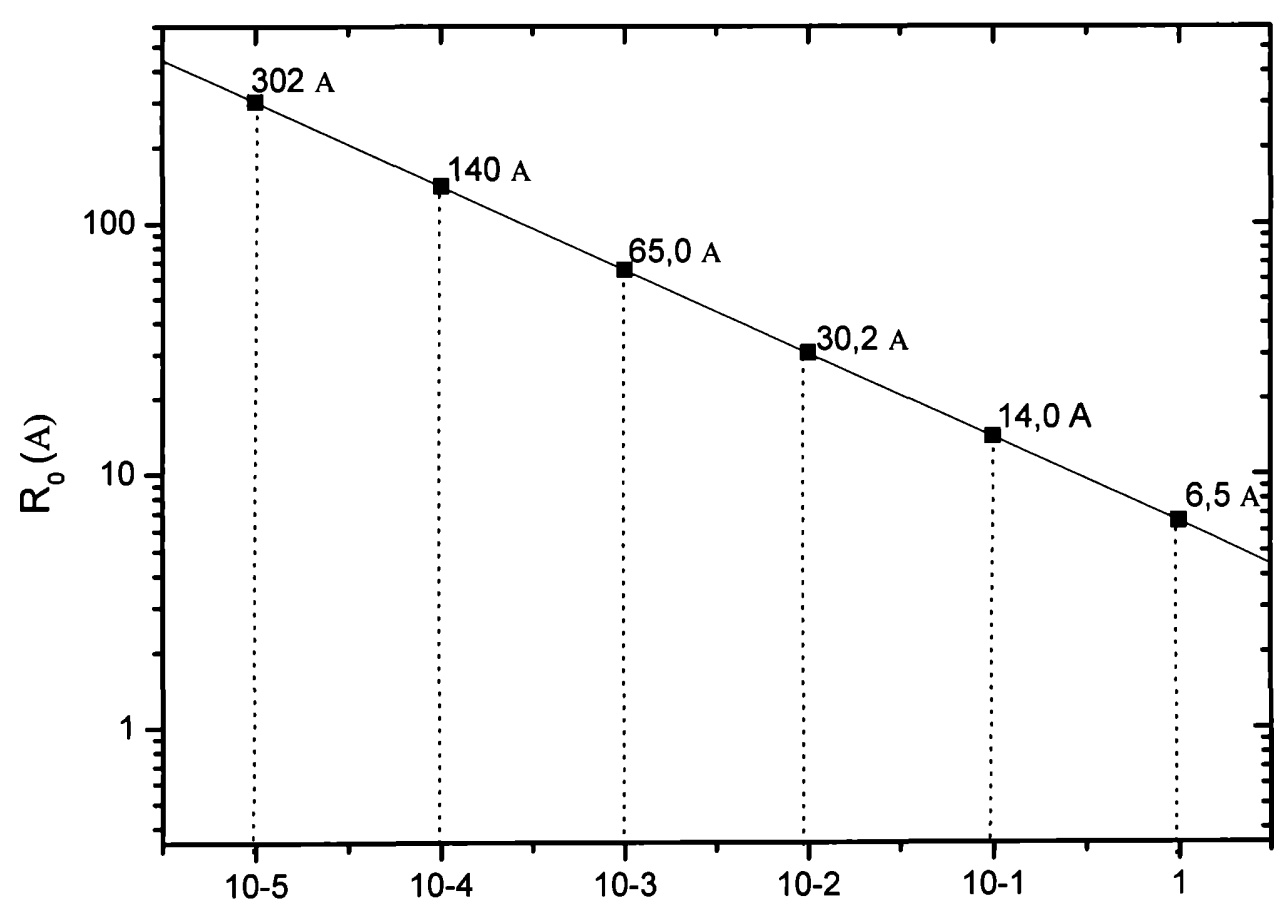

(A)

Figura 2: Relación entre la concentración de aceptor y la separación promedio entre donor y aceptor en solución rígida. 


\section{v. Teoría de Förster en polímeros}

Cuando un cromóforo se encuentra inmovilizado en un esqueleto polimérico, el decaimiento de la luminiscencia se encuentra bajo la influencia de la interacción con la matriz polimérica. Por lo tanto se puede suponer que el perfil de decaimiento de una molécula es el promedio de una distribución de perfiles de velocidades de relajación, por lo que dicho decaimiento será no exponencial. La probabilidad dependiente del tiempo $\mathrm{P}(\mathrm{t})$ de que un cromóforo se encuentre en el estado excitado a un tiempo t luego de la excitación está dada por la resolución de la siguiente ecuación diferencial:

$$
-\frac{d P(t)}{d t}=P(t)\left(k_{r}+k_{n r}+k_{E T}\right)
$$

Donde $k_{\mathrm{r}}$, es la constate de velocidad radiativa: $k_{\mathrm{nr}}$, es la constante de relajación no radiativa del cromóforo y $k_{\mathrm{ET}}$ es la constante de quenching por transferencia de energía.

En la evaluación de $k_{\mathrm{ET}}$, se supone que el quenching del donor D se lleva a cabo por interacción con las regiones vecinas del polímero que contienen moléculas de aceptor A. La constante de quenching para el i-ésimo donor $\mathrm{D}_{\mathrm{i}}$, es la suma sobre las distancias con $\mathrm{j}$ sitios quenching:

$$
k_{E T}\left(D_{i}\right)=\frac{1}{\tau_{D}} \sum\left(\frac{R_{F}}{R\left(D_{i, j}\right)}\right)^{6}
$$


Al sustituir $k_{\mathrm{ET}}$ en la ecuación 31, la ecuación diferencial puede ser resuelta. Como la distancia R entre D y A depende de la conformación de la cadena, debe considerarse la suma sobre todas las contibuciones posibles.

La solución de la ecuación diferencial correspondiente a una interacción dipolo-dipolo en tres dimensiones está dada por. ${ }^{12-13}$

$$
N_{t}=N_{0} \exp \left[-\frac{t}{\tau_{D}}-a \sqrt{t / \tau_{D}}\right]
$$

Donde $\mathrm{N}_{\mathrm{t}}$ es el número de moléculas que permanecen en estado excitado al tiempo t, y $a$ es una parámetro proporcional a la densidad de sitios quenching aceptores, y puede ser calculado con la siguiente expresión;

$$
a=\frac{4}{3} \pi^{3 / 2} \rho R_{F}^{3}
$$

Donde $\rho$ es el número de sitios quenching por unidad de volumen. El número de sitios quenching dentro del radio crítico está dado por:

$$
N=\frac{a}{\sqrt{\pi}}
$$

La ecuación 33 fue utilizada para ajustar las trazas de emisión resuelta en el tiempo de los polímeros mixtos, ver mas adelante. 


\section{Resultados experimentales}

\section{i. Espectroscopía de emisión estacionaria}

En la figura 3 pueden observarse los espectros de emisión de los cinco polímeros que se han sintetizado.

Todos los espectros fueron tomados sobre soluciones preparadas en $\mathrm{CH}_{3} \mathrm{CN}$ con una absorbancia A: 0.05 , las cuales fueron deaereadas con $\mathrm{N}_{2}$ ultrapuro durante 15 minutos antes de realizar la medida.

En todos los casos se irradia con luz de la misma longitud de onda de excitación $\lambda_{\text {exc }}=$ $380 \mathrm{~nm}$, con el fin de poblar mayoritariamente el estado excitado de transferencia de carga metal-ligando TCML (banda $\mathrm{d}_{\pi} \rightarrow \pi^{*}$ ).

Puede observarse que la longitud de onda del máximo de emisión se centra en $530 \mathrm{~nm}$

en los polímeros $\left\{(\mathrm{vpy})_{2} \operatorname{vpyRe}(\mathrm{CO})_{3}(\text { tmephen })^{+}\right\}_{\mathrm{n} \sim 200}$;

$\left\{(\text { vpy })_{2} \text { vpyRe }(\mathrm{CO})_{3}(\text { tmephen })^{+}\right\}_{\mathrm{n} \sim 180}\left\{(\text { vpy })_{2} \text { vpyRe }(\mathrm{CO})_{3}\left(\mathrm{NO}_{2}-\text { phen }\right)^{+}\right\}_{\mathrm{m} \sim 200} ;$

$\left\{(\mathrm{vpy})_{2} \operatorname{vpyRe}(\mathrm{CO})_{3}(\text { tmephen })^{+}\right\}_{\mathrm{n} \sim 150}\left\{(\mathrm{vpy})_{2} \mathrm{vpyRe}(\mathrm{CO})_{3}\left(\mathrm{NO}_{2}-\mathrm{phen}\right)^{+}\right\}_{\mathrm{m} \sim 50} ;$

$\left\{(\mathrm{vpy})_{2} \mathrm{vpyRe}(\mathrm{CO})_{3}(\text { tmephen })^{+}\right)_{\mathrm{n} \sim 100}\left\{(\mathrm{vpy})_{2} \mathrm{vpyRe}(\mathrm{CO})_{3}\left(\mathrm{NO}_{2}-\mathrm{phen}\right)^{+}\right\}_{\mathrm{m} \sim 100}$, y en el polímero $\left\{(\mathrm{vpy})_{2} \mathrm{Re}(\mathrm{CO})_{3}\left(\mathrm{NO}_{2} \text {-phen }\right)^{+}\right\}_{n \sim 200}$ la emisión es inexistente.

La banda no posee estructura, lo cual está en concordancia con un estado emisor de TCML, en este caso $\mathrm{Re} \rightarrow$ tmephen, ya que como lo demuestra la falta de emisión del polímero $\left\{(\text { vpy })_{2} \operatorname{Re}(\mathrm{CO})_{3}\left(\mathrm{NO}_{2} \text {-phen }\right)^{+}\right\}_{n \sim 200}$ el estado de $\mathrm{TCML}_{\mathrm{Re} \rightarrow \mathrm{NO} 2 \text {-phen }}$ decae principalmente no radiativamente. 


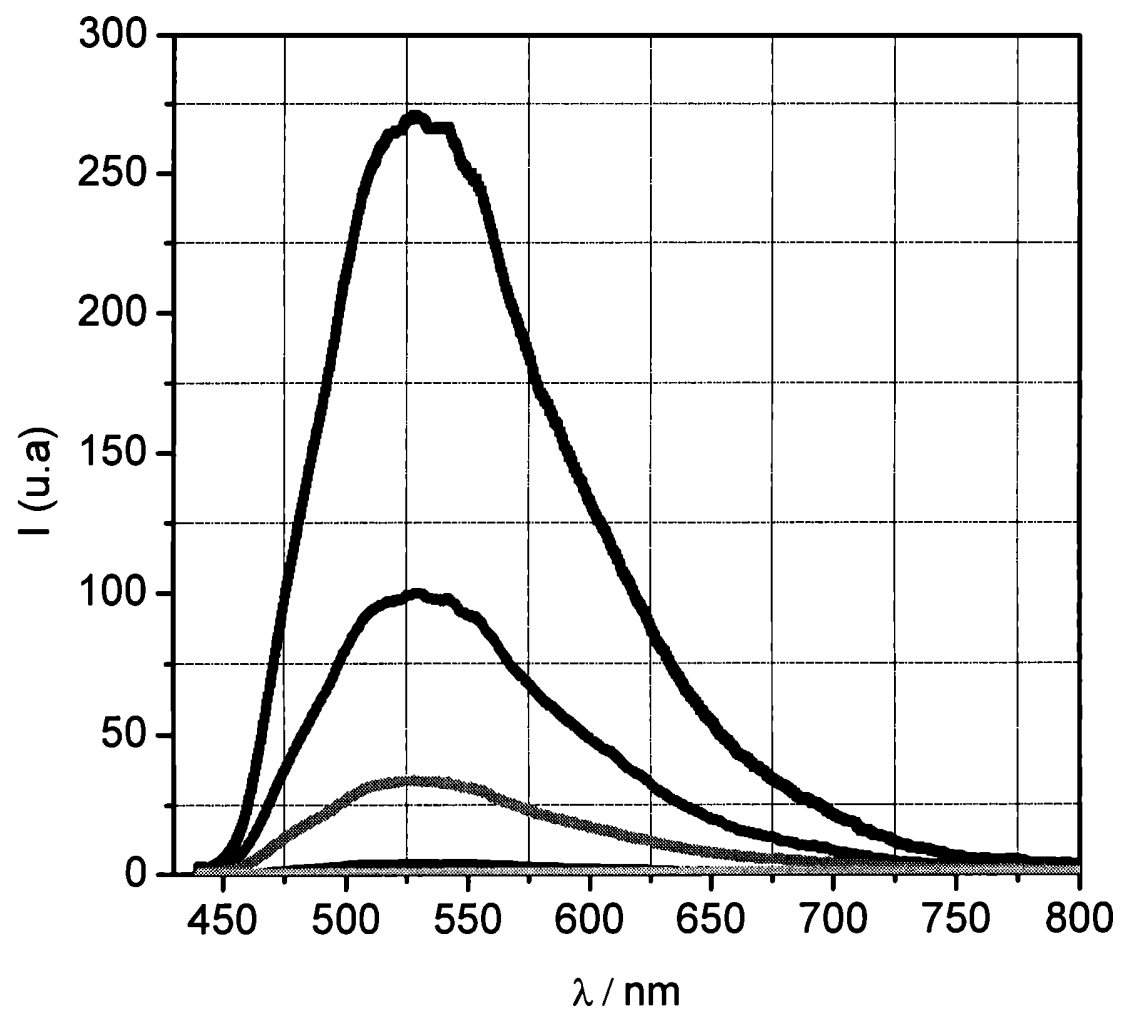

Figura 3: Espectro de emisión de los polímeros en $\mathrm{CH}_{3} \mathrm{CN}$ a temperatura ambiente deaereados con $\mathrm{N}_{2}$ y con A: 0.05 .

- $\left\{(\mathrm{vpy})_{2} \mathrm{vpyRe}(\mathrm{CO})_{3}(\text { tmephen })^{+}\right\}_{\mathrm{n} \sim 200}$

- $\left\{(\mathrm{vpy})_{2} \mathrm{vpy} \operatorname{Re}(\mathrm{CO})_{3}(\mathrm{tm} \text { ephen })^{+}\right\}_{\mathrm{n} \sim 180}\left\{(\mathrm{vpy})_{2} \mathrm{vpyRe}(\mathrm{CO})_{3}\left(\mathrm{NO}_{2}-\text { phen }\right)^{+}\right\}_{\mathrm{m} \sim 20}$

$\left\{(\mathrm{vpy})_{2} \mathrm{vpy} \operatorname{Re}(\mathrm{CO})_{3}(\mathrm{tmephen})^{+}\right\}_{\mathrm{n} \sim 150}\left\{(\mathrm{vpy})_{2} \mathrm{vpy} \operatorname{Re}(\mathrm{CO})_{3}\left(\mathrm{NO}_{2}-\text { phen }\right)^{+}\right\}_{\mathrm{m}-50}$,

$\nabla\left\{(\mathrm{vpy})_{2} \mathrm{vpyRe}(\mathrm{CO})_{3}(\text { tmephen })^{+}\right)_{\mathrm{n} \sim 100}\left\{(\mathrm{vpy})_{2} \mathrm{vpyRe}(\mathrm{CO})_{3}\left(\mathrm{NO}_{2}-\text { phen }\right)^{+}\right\}_{\mathrm{m} \sim 100}$, $\left\{(\mathrm{vpy})_{2} \mathrm{vpyRe}(\mathrm{CO})_{3}\left(\mathrm{NO}_{2}-\text { phen }\right)^{+}\right\}_{\mathrm{n} \sim 200}$ 


\section{ii. Espectroscopia de emisión estacionaria de mezclas de polímeros}

Además, se realizaron los espectros de emisión de soluciones preparadas a partir de la mezcla de los polímeros $\left\{(\mathrm{vpy})_{2} \mathrm{vpyRe}(\mathrm{CO})_{3}(\text { tmephen })^{+}\right\}_{\mathrm{n} \sim 200}$ y $\left\{(\mathrm{vpy})_{2} \operatorname{Re}(\mathrm{CO})_{3}\left(\mathrm{NO}_{2^{-}}\right.\right.$ phen $\left.)^{+}\right\}_{n-200}$ en la relación adecuada para obtener las mismas relaciones de cromóforos que en los tres polímeros mixtos, es decir:

$\operatorname{Re}(\mathrm{CO})_{3}(\text { tmephen })^{+} / \operatorname{Re}(\mathrm{CO})_{3}\left(\mathrm{NO}_{2} \text {-phen }\right)^{+}: 180 / 20 ; 150 / 50 ; 100 / 100$.

Todas las soluciones se prepararon en $\mathrm{CH}_{3} \mathrm{CN}$, y los espectros fueron realizados en las mismas condiciones que los cinco polímeros sintetizados, $\lambda_{\mathrm{exc}}=380 \mathrm{~nm} \mathrm{~A}=0.05 \mathrm{y} \mathrm{a}$ temperatura ambiente.

Dichos espectros pueden observarse en la figura 4. 


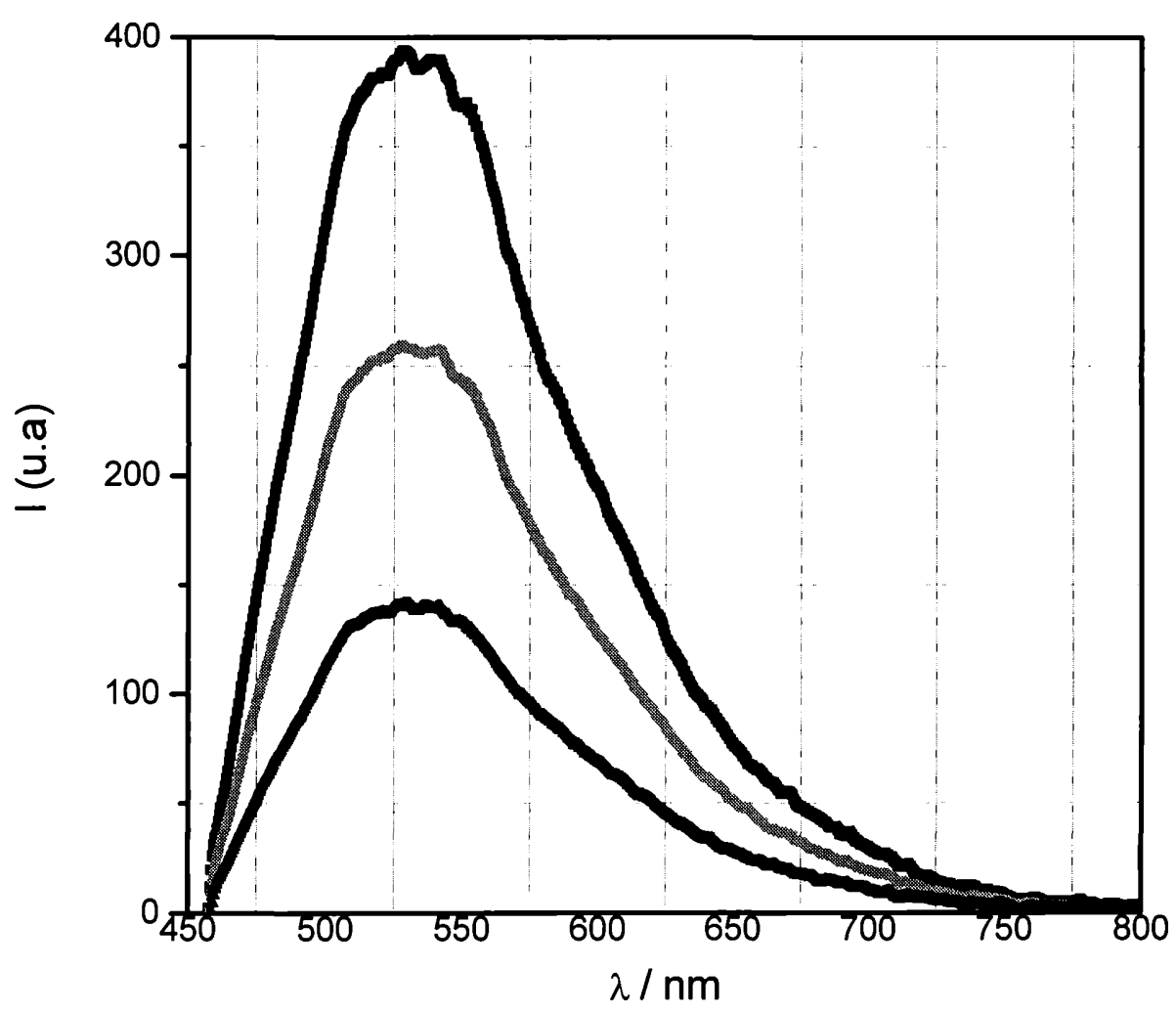

Figura 4: Espectro de emisión de las mezcla molares de los polímeros

$\left\{(\mathrm{vpy})_{2} \text { vpyRe}(\mathrm{CO})_{3}(\text { tmephen })^{+}\right\}_{\mathrm{n} \sim 200}$ y $\left\{(\mathrm{vpy})_{2} \mathrm{vpyRe}(\mathrm{CO})_{3}\left(\mathrm{NO}_{2}-\mathrm{phen}\right)^{+}\right\}_{\mathrm{m} \sim 20}$ en

$\mathrm{CH}_{3} \mathrm{CN}$ a temperatura ambiente deaereados con $\mathrm{N}_{2}$ y con $\mathrm{A}: 0.05$.

- $\left.\left\{(\mathrm{vpy})_{2} \text { vpyRe(CO) }\right)_{3}(\text { tmephen })^{+}\right\}_{\mathrm{n} \sim 200}\left\{(\mathrm{vpy})_{2} \mathrm{vpyRe}(\mathrm{CO})_{3}\left(\mathrm{NO}_{2}-\mathrm{phen}\right)^{+}\right\}_{\mathrm{m}-200} 180 / 20$

- $\left\{(\mathrm{vpy})_{2} \mathrm{vpyRe}(\mathrm{CO})_{3}(\text { tmephen })^{+}\right\}_{\mathrm{n} \sim 200}\left\{(\mathrm{vpy})_{2} \mathrm{vpyRe}(\mathrm{CO})_{3}\left(\mathrm{NO}_{2}-\text { phen }\right)^{+}\right\}_{\mathrm{m}-200} 150 / 50$

$\Delta\left\{(\text { vpy })_{2} \operatorname{vpyRe}(\mathrm{CO})_{3}(\text { tmephen })^{+}\right\}_{\mathrm{n} \sim 200}\left\{(\mathrm{vpy})_{2} \mathrm{vpyRe}(\mathrm{CO})_{3}\left(\mathrm{NO}_{2}-\mathrm{phen}\right)^{+}\right\}_{\mathrm{m} \sim 200} 100 / 100$ 


\section{iii. Rendimiento cuántico de emisión}

Se determinó el rendimiento cuántico de emisión relativo de los cinco polímeros sintetizados y de las soluciones de polímeros mezcla, utilizando rodamina B como referencia. Para lo cual se utilizó la ecuación:

$$
\phi_{e m, p o l}=\left(\frac{A_{r e f}}{A_{p o l}}\right)\left(\frac{I_{p o l}}{I_{r e f}}\right)\left(\frac{n_{p o l}}{n_{r e f}}\right)^{2} \phi_{e m, r e f}
$$

Las soluciones de polímero y las mezclas se prepararon todas en acetonitrilo, y la solución de rodamina B se preparó en etanol absoluto. Los experimentos fueron realizados utilizando en todos los casos $\lambda_{\text {exc }}=380 \mathrm{~nm}, \mathrm{~A}=0.1 \mathrm{y}$ a temperatura ambiente.

Los resultados se muestran en las tablas 1 y 2 .

\begin{tabular}{|l|c|}
\hline Polimero & $\phi_{\mathrm{em}}$ \\
\hline$\left\{(\mathrm{vpy})_{2} \operatorname{Re}(\mathrm{CO})_{3}\left(\mathrm{NO}_{2} \text {-phen }\right)^{+}\right\}_{\mathrm{n}-200}$ & $<1 \times 10^{-4}$ \\
\hline$\left\{(\mathrm{vpy})_{2} \mathrm{vpyRe}(\mathrm{CO})_{3}(\text { tmephen })^{+}\right\}_{\mathrm{n}-200}$ & 0.0347 \\
\hline$\left\{(\mathrm{vpy})_{2} \mathrm{vpyRe}(\mathrm{CO})_{3}(\text { tmephen })^{+}\right\}_{\mathrm{n} \sim 80}\left\{(\mathrm{vpy})_{2} \mathrm{vpyRe}(\mathrm{CO})_{3}\left(\mathrm{NO}_{2} \text {-phen }\right)^{+}\right\}_{\mathrm{m}-20}$ & 0.0130 \\
\hline$\left\{(\mathrm{vpy})_{2} \mathrm{vpyRe}(\mathrm{CO})_{3}(\text { tmephen })^{+}\right\}_{\mathrm{n}-150}\left\{(\mathrm{vpy})_{2} \mathrm{vpyRe}(\mathrm{CO})_{3}\left(\mathrm{NO}_{2} \text {-phen }\right)^{+}\right\}_{\mathrm{m} \sim 50}$ & 0.0038 \\
\hline$\left\{(\mathrm{vpy})_{2} \mathrm{vpyRe}(\mathrm{CO})_{3}(\text { tmephen })^{+}\right)_{\mathrm{n}-100}\left\{(\mathrm{vpy})_{2} \mathrm{vpyRe}(\mathrm{CO})_{3}\left(\mathrm{NO}_{2} \text {-phen }\right)^{+}\right\}_{\mathrm{m}-100}$ & 0.0007 \\
\hline
\end{tabular}

Tabla 1: Rendimiento cuántico de emisión de los polímeros

$\left\{(\mathrm{vpy})_{2} \mathrm{vpyRe}(\mathrm{CO})_{3} \text { (tmephen }^{+}\right\}_{\mathrm{n}-200}$, $\left\{(\text { vpy })_{2} \text { vpyRe }(\mathrm{CO})_{3}(\text { tmephen })^{+}\right\}_{\mathrm{n}-180}\left\{(\mathrm{vpy})_{2} \mathrm{vpyRe}(\mathrm{CO})_{3}\left(\mathrm{NO}_{2} \text {-phen }\right)^{+}\right\}_{\mathrm{m}-20}$, $\left\{(\mathrm{vpy})_{2} \mathrm{vpyRe}(\mathrm{CO})_{3}(\text { tmephen })^{+}\right\}_{\mathrm{n} \sim 150}\left\{(\mathrm{vpy})_{2} \mathrm{vpy} \operatorname{Re}(\mathrm{CO})_{3}\left(\mathrm{NO}_{2} \text {-phen }\right)^{+}\right\}_{\mathrm{m} \sim 50}$, $\left.\left.\left\{(\text { vpy })_{2} \text { vpyRe(CO }\right)_{3}(\text { tmephen })^{+}\right)_{n-100}\left\{(\text { vpy })_{2} \text { vpyRe(CO }\right)_{3}\left(\mathrm{NO}_{2} \text {-phen }\right)^{+}\right\}_{\mathrm{m} \sim 100}$, $\left\{(\text { vpy })_{2} \text { vpyRe }(\mathrm{CO})_{3}\left(\mathrm{NO}_{2} \text {-phen }\right)^{+}\right\}_{\mathrm{n}-200}$ $\mathrm{En} \mathrm{CH}_{3} \mathrm{CN}, \lambda_{\text {exc }}=380 \mathrm{~nm}$ 


\begin{tabular}{|c|c|}
\hline Polímero mezcla & $\phi_{\mathrm{mm}}$ \\
\hline$\left\{(\mathrm{vpy})_{2} \mathrm{vpyRe}(\mathrm{CO})_{3}(\text { tmephen })^{+}\right\}_{200} /\left\{(\mathrm{vpy})_{2} \mathrm{vpyRe}(\mathrm{CO})_{3}\left(\mathrm{NO}_{2}-\text { phen }\right)^{+}\right\}_{200}: 180 / 20$ & 0.0310 \\
\hline$\left\{(\mathrm{vpy})_{2} \mathrm{vpyRe}(\mathrm{CO})_{3}(\text { tmephen })^{+}\right\}_{200} /\left\{(\mathrm{vpy})_{2} \mathrm{vpyRe}(\mathrm{CO})_{3}\left(\mathrm{NO}_{2}-\text { phen }\right)^{+}\right\}_{200}: 150 / 50$ & 0.0260 \\
\hline$\left\{(\mathrm{vpy})_{2} \mathrm{vpyRe}(\mathrm{CO})_{3}(\text { tmephen })^{+}\right)_{200} /\left\{(\mathrm{vpy})_{2} \mathrm{vpyRe}(\mathrm{CO})_{3}\left(\mathrm{NO}_{2}-\mathrm{phen}\right)^{+}\right\}_{200}: 100 / 100$ & 0.0125 \\
\hline
\end{tabular}

Tabla 2: Rendimiento cuántico de emisión $\phi_{\mathrm{mm}}$, de las mezclas molares de polímeros:

$\left\{(\mathrm{vpy})_{2} \mathrm{vpy} \operatorname{Re}(\mathrm{CO})_{3}(\mathrm{tmephen})^{+}\right\}_{\mathrm{n} \sim 200}\left\{(\mathrm{vpy})_{2} \mathrm{vpyRe}(\mathrm{CO})_{3}\left(\mathrm{NO}_{2}-\text { phen }\right)^{+}\right\}_{\mathrm{m} \sim 200} 180 / 20$

$\left\{(\mathrm{vpy})_{2} \mathrm{vpyRe}(\mathrm{CO})_{3}(\text { tmephen })^{+}\right\}_{\mathrm{n}-200}\left\{(\mathrm{vpy})_{2} \mathrm{vpyRe}(\mathrm{CO})_{3}\left(\mathrm{NO}_{2}-\mathrm{phen}\right)^{+}\right\}_{\mathrm{m}-200} 150 / 50$

$\left\{(\mathrm{vpy})_{2} \mathrm{vpyRe}(\mathrm{CO})_{3}(\text { tmephen })^{+}\right\}_{\mathrm{n}-200}\left\{(\mathrm{vpy})_{2} \mathrm{vpyRe}(\mathrm{CO})_{3}\left(\mathrm{NO}_{2}-\text { phen }\right)^{+}\right\}_{\mathrm{m} \sim 200} 100 / 100$

$\mathrm{En} \mathrm{CH}_{3} \mathrm{CN}, \lambda_{\mathrm{exc}}=380 \mathrm{~nm}$

Los redimientos cuánticos de emisión de los polímeros mixtos, considerando ausencia de quenching, y teniendo en cuenta que el único estado emisor es el estado TCML $\mathrm{Re} \rightarrow$ tmphen pueden calcularse a partir de la siguiente expresión:

$$
\begin{aligned}
& \phi_{e m}=\left(\frac{A}{A_{T}}\right) \phi_{e m}^{0} \\
& \frac{A}{A_{T}}=\frac{n \varepsilon_{\lambda, n}}{n \varepsilon_{\lambda, n}+m \varepsilon_{\lambda, m}}
\end{aligned}
$$

donde A es la absorbancia de $\left\{(\mathrm{vpy})_{2} \operatorname{vpyRe}(\mathrm{CO})_{3}(\text { tmephen })^{+}\right\}_{\mathrm{n} \sim 200}$ a la longitud de onda $\lambda$, $\mathrm{A}_{\mathrm{T}}$ es la absorbancia total de la solución y $\varepsilon_{\lambda, \mathrm{n}}$ son los coeficientes de extinción molar de 
$\left\{(\text { vpy })_{2} \text { vpyRe }(\mathrm{CO})_{3}(\text { tmephen })^{+}\right\}_{\mathrm{n} \sim 200}$ y $\left\{(\mathrm{vpy})_{2} \mathrm{vpyRe}(\mathrm{CO})_{3}\left(\mathrm{NO}_{2} \text {-phen }\right)^{+}\right\}_{\mathrm{n} \sim 200}$ a la longitud de onda $\lambda$, respectivamente, $\phi_{e m}^{0}$ es el rendimiento cuántico de emisión de $\left\{(\text { vpy })_{2} \text { vpy } \operatorname{Re}(\mathrm{CO})_{3}(\text { tmephen })^{+}\right\}_{\mathrm{n} \sim 200}$.

Tomando los valores de $5.5 \times 10^{5}$ y $7.8 \times 10^{5} \mathrm{M}^{-1} \mathrm{~cm}^{-1}$ para los coeficientes de extinción molar de los polímeros $\left\{(\mathrm{vpy})_{2} \mathrm{vpyRe}(\mathrm{CO})_{3}(\mathrm{tmephen})^{+}\right\}_{\mathrm{n} \sim 200} \quad$ y $\left\{(\mathrm{vpy})_{2} \operatorname{Re}(\mathrm{CO})_{3}\left(\mathrm{NO}_{2}-\right.\right.$ phen $\left.)^{+}\right\}_{\mathrm{n} \sim 200}$ a $\lambda: 380 \mathrm{~nm}$ se pueden calcular los $\phi_{\mathrm{em}}$ que se muestran en la tabla 3 .

\begin{tabular}{|l|c|}
\hline Polimero mixto & $\phi_{\text {em,calc }}$ \\
\hline$\left\{(\mathrm{vpy})_{2} \mathrm{vpyRe}(\mathrm{CO})_{3}(\text { tmephen })+\right\}_{n \sim 180}\left\{(\mathrm{vpy})_{2} \mathrm{vpyRe}(\mathrm{CO})_{3}\left(\mathrm{NO}_{2}-\text { phen }\right)+\right\}_{\mathrm{m} \sim 20}$ & 0.0298 \\
\hline$\left\{(\mathrm{vpy})_{2} \mathrm{vpyRe}(\mathrm{CO})_{3}(\text { tmephen })+\right\}_{n \sim 150}\left\{(\mathrm{vpy})_{2} \mathrm{vpyRe}(\mathrm{CO})_{3}\left(\mathrm{NO}_{2}-\text { phen }\right)+\right\}_{\mathrm{m}-50}$ & 0.0236 \\
\hline$\left\{(\mathrm{vpy})_{2} \mathrm{vpyRe}(\mathrm{CO})_{3}(\text { tmephen })+\right\}_{n \sim 100}\left\{(\mathrm{vpy})_{2} \mathrm{vpyRe}(\mathrm{CO})_{3}\left(\mathrm{NO}_{2}-\text { phen }\right)+\right\}_{\mathrm{m} \sim 100}$ & 0.0142 \\
\hline
\end{tabular}

Tabla 3: Rendimiento cuántico de emisión de los polímeros mixtos $\left\{(\text { vpy })_{2} \text { vpyRe }(\mathrm{CO})_{3}(\text { tm ephen })^{+}\right\}_{\mathrm{n}-180}\left\{(\mathrm{vpy})_{2} \operatorname{vpyRe}(\mathrm{CO})_{3}\left(\mathrm{NO}_{2} \text {-phen }\right)^{+}\right\}_{\mathrm{m}-20}$, $\left\{(\mathrm{vpy})_{2} \mathrm{vpyRe}(\mathrm{CO})_{3}(\text { tmephen })^{+}\right\}_{\mathrm{n}-150}\left\{(\mathrm{vpy})_{2} \mathrm{vpyRe}(\mathrm{CO})_{3}\left(\mathrm{NO}_{2}-\mathrm{phen}\right)^{+}\right\}_{\mathrm{m}-50}$ $\left\{(\mathrm{vpy})_{2} \mathrm{vpyRe}(\mathrm{CO})_{3}(\text { tmephen })^{+}\right)_{\mathrm{n} \sim 100}\left\{(\mathrm{vpy})_{2} \mathrm{vpy} \operatorname{Re}(\mathrm{CO})_{3}\left(\mathrm{NO}_{2}-\mathrm{phen}\right)^{+}\right\}_{\mathrm{m} \sim 100}$ Calculados a partir de la expresión : $\phi_{e m}=\left(\frac{A}{A_{T}}\right) \phi_{e m}^{0}$

\section{iv. Espectroscopia de absorción resuelta en el tiempo}

El espectro de absorción del estado excitado ó transiente, del polímero $\left\{(\mathrm{vpy})_{2} \operatorname{Re}(\mathrm{CO})_{3}\left(\mathrm{NO}_{2} \text {-phen }\right)^{+}\right\}_{\mathrm{n}-200}$ fue registrado con el instrumento de láser flash fotólisis en la escala de los picosegundos, que ha sido descripto en la parte I capítulo IV, con una longitud 
de onda de excitación de $387 \mathrm{~nm}$. Las soluciones del polímero fueron preparadas en acetonitrilo y la concentración utilizada fue de $1.5 \times 10^{-6} \mathrm{M}\left([\operatorname{Re}]: 3 \times 10^{-4} \mathrm{M}\right)$.

El espectro de absorción del transiente tomando el $\Delta \mathrm{A}$ a 2, 4, 60, 100 y 200 ps luego de la irradiación del láser versus la longitud de onda se muestra en la figura 5. El decaimiento observado es monoexponencial y el tiempo de vida fue de $\tau=0.23 \mathrm{~ns}$.

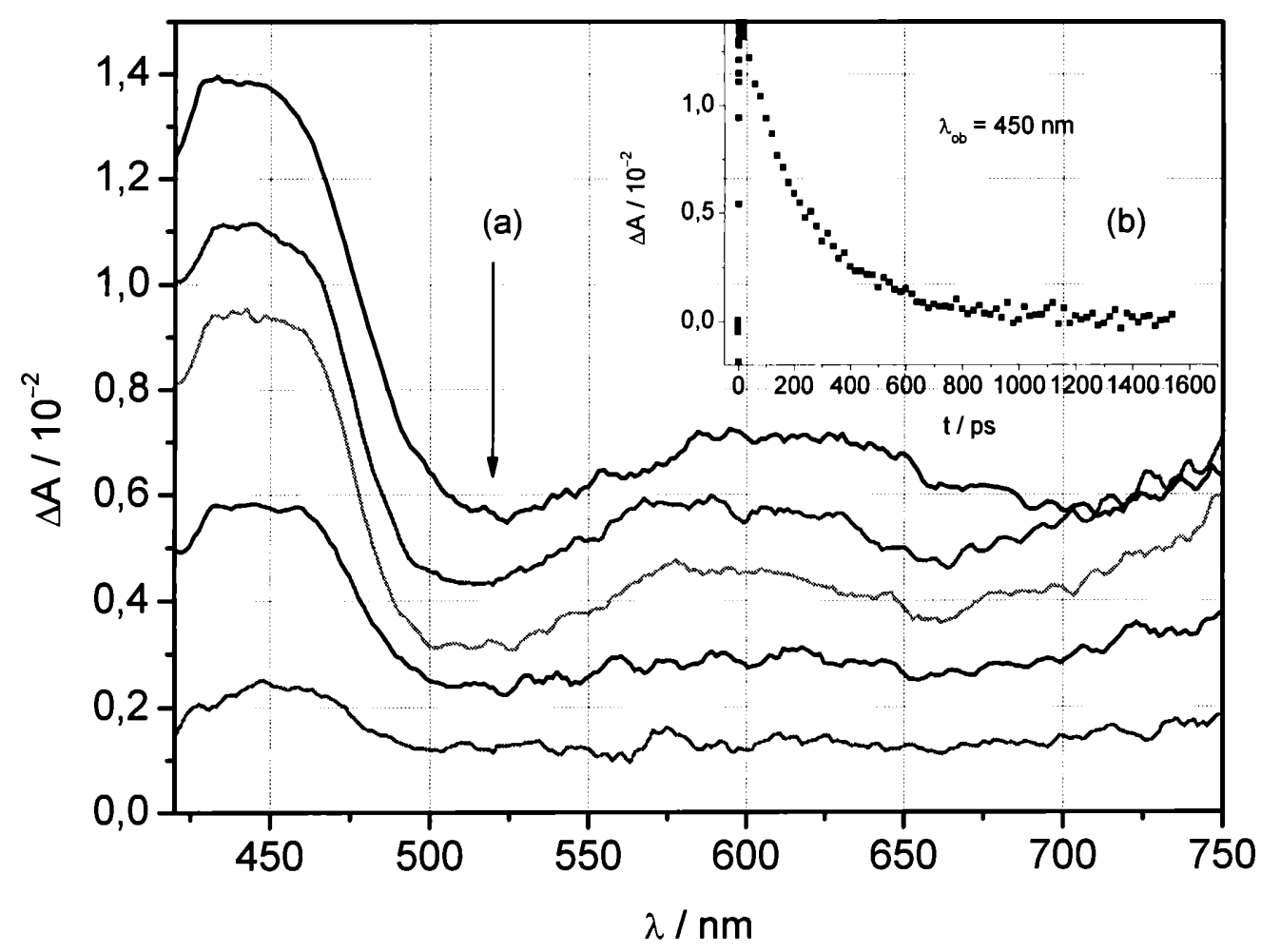

Figura 5. a. Espectro de absorción resuelta en el tiempo en una escala femtosegundonanosegundos luego de la irradiación flash con longitud de onda de $387 \mathrm{~nm}$ de soluciones en acetonitrilo del polímero $\left\{(\mathrm{vpy})_{2} \operatorname{Re}(\mathrm{CO})_{3}\left(\mathrm{NO}_{2} \text {-phen }\right)^{+}\right\}_{\mathrm{n}-200}$. Los espectros fueron tomados luego de 2, 4, 60, 100, y 200 ps del pulso del láser.. El espectro evoluciona en la dirección de la flecha. b. traza osciloscópica típica que revela el decaimiento monoexponencial a $\lambda_{o b}=450 \mathrm{~nm}$. 
Los espectros de absorción del transiente de los polímeros: $\left\{(\mathrm{vpy})_{2} \operatorname{vpyRe}(\mathrm{CO})_{3}(\text { tmephen })^{+}\right\}_{\mathrm{n} \sim 200}$

$\left\{(\text { vpy })_{2} \text { vpyRe }(\mathrm{CO})_{3}(\text { tmephen })^{+}\right\}_{\mathrm{n} \sim 180}\left\{(\mathrm{vpy})_{2} \operatorname{vpyRe}(\mathrm{CO})_{3}\left(\mathrm{NO}_{2}-\text { phen }\right)^{+}\right\}_{\mathrm{m} \sim 20}$

$\left\{(\mathrm{vpy})_{2} \mathrm{vpyRe}(\mathrm{CO})_{3}(\text { tmephen })^{+}\right\}_{\mathrm{n} \sim 150}\left\{(\mathrm{vpy})_{2} \operatorname{vpyRe}(\mathrm{CO})_{3}\left(\mathrm{NO}_{2}-\text { phen }\right)^{+}\right\}_{\mathrm{m} \sim 50}$

$\left\{(\text { vpy })_{2} \text { vpyRe }(\mathrm{CO})_{3}(\text { tmephen })^{+}\right)_{\mathrm{n} \sim 100}\left\{(\text { vpy })_{2} \operatorname{vpyRe}(\mathrm{CO})_{3}\left(\mathrm{NO}_{2}-\text { phen }\right)^{+}\right\}_{\mathrm{m} \sim 100}$

fueron realizados con el láser de excimero de XeF ya descripto anteriormente $\left(\lambda_{\mathrm{exc}}=351 \mathrm{~nm}\right)$.

Estos transientes decayeron en la escala de tiempo entre los ns y los $\mu$ s.

Las trazas fueron ajustadas con un decaimento biexponencial (figura 6) después del pulso de irradiación, que se completó en la escala de los microsegundos. Los valores de los tiempos de vida se muestran en la tabla 4 .

Los espectros del transiente para cada uno de los polímeros, se obtuvieron graficando el $\Delta \mathrm{A}_{\max }$ de la curva de decaimiento correspondiente versus la longitud de onda (figura 7). 

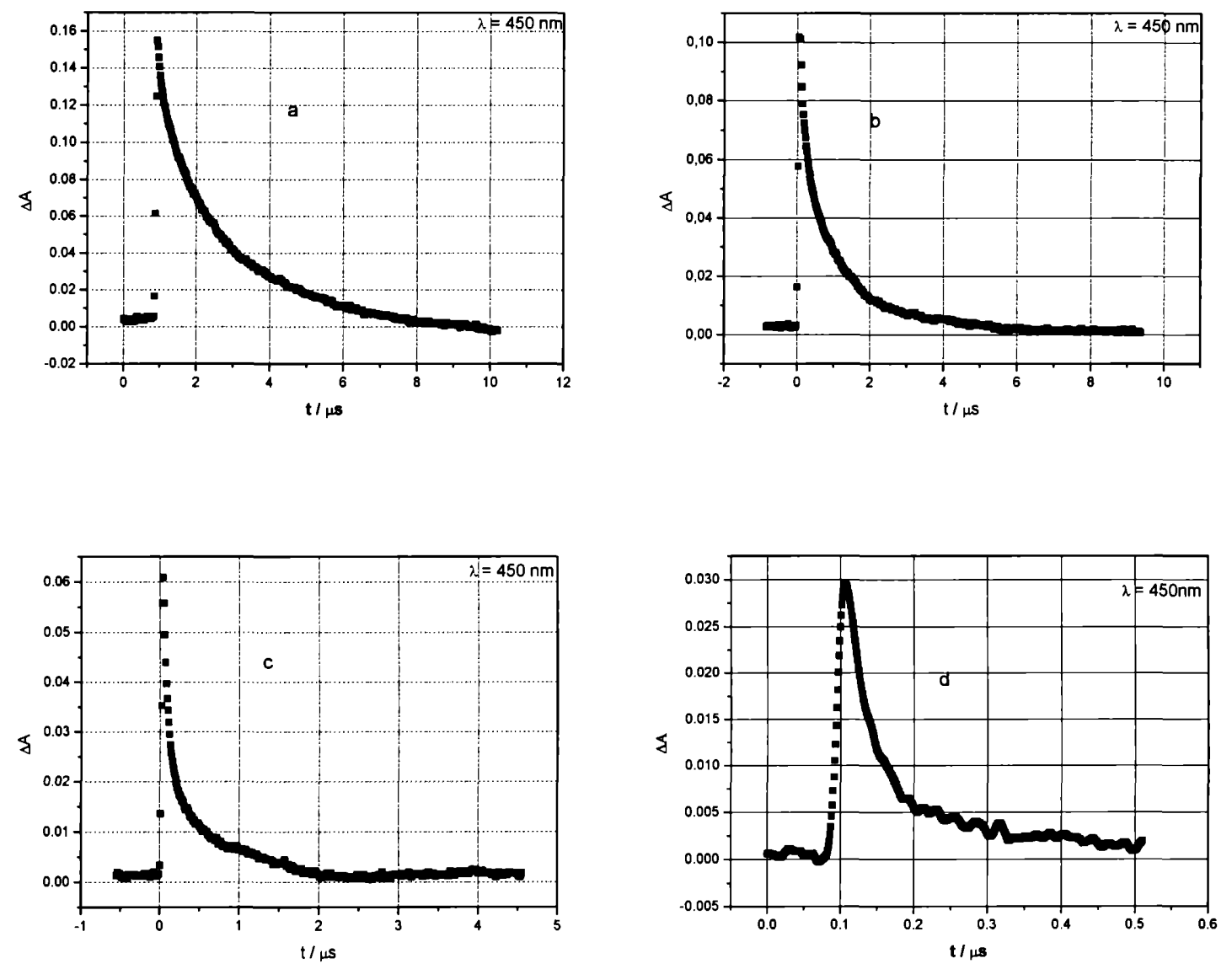

Figura 6: Trazas de los decaimientos de absorción de los transientes de los polímeros a: $\left\{(\mathrm{vpy})_{2} \mathrm{vpy} \operatorname{Re}(\mathrm{CO})_{3}(\mathrm{tmephen})^{+}\right\}_{\mathrm{n} \sim 200}$;

b: $\left.\left\{(\text { vpy })_{2} \text { vpyRe(CO) }\right)_{3}(\text { tmephen })^{+}\right\}_{\mathrm{n} \sim 180}\left\{(\mathrm{vpy})_{2} \mathrm{vpyRe}(\mathrm{CO})_{3}\left(\mathrm{NO}_{2}-\mathrm{phen}\right)^{+}\right\}_{\mathrm{m}-20}$

c: $\left\{(\mathrm{vpy})_{2} \mathrm{vpy} \operatorname{Re}(\mathrm{CO})_{3}(\mathrm{tmephen})^{+}\right\}_{\mathrm{n} \sim 150}\left\{(\mathrm{vpy})_{2} \mathrm{vpyRe}(\mathrm{CO})_{3}\left(\mathrm{NO}_{2}-\mathrm{phen}\right)^{+}\right\}_{\mathrm{m} \sim 50} \mathrm{y}$

$\mathrm{d}\left\{(\mathrm{vpy})_{2} \mathrm{vpyRe}(\mathrm{CO})_{3}(\text { tmephen })^{+}\right)_{\mathbf{n} \sim 100}\left\{(\mathrm{vpy})_{2} \mathrm{vpyRe}(\mathrm{CO})_{3}\left(\mathrm{NO}_{2} \text {-phen }\right)^{+}\right\}_{\mathrm{m} \sim 100}$

registrados con un láser excimero $\mathrm{XeF}$ de $\lambda_{\text {exc }}: 351 \mathrm{~nm}$ sobre soluciones preparadas en acetonitrilo y deaereadas con $\mathrm{N}_{2}$ ultrapuro a T ambiente 


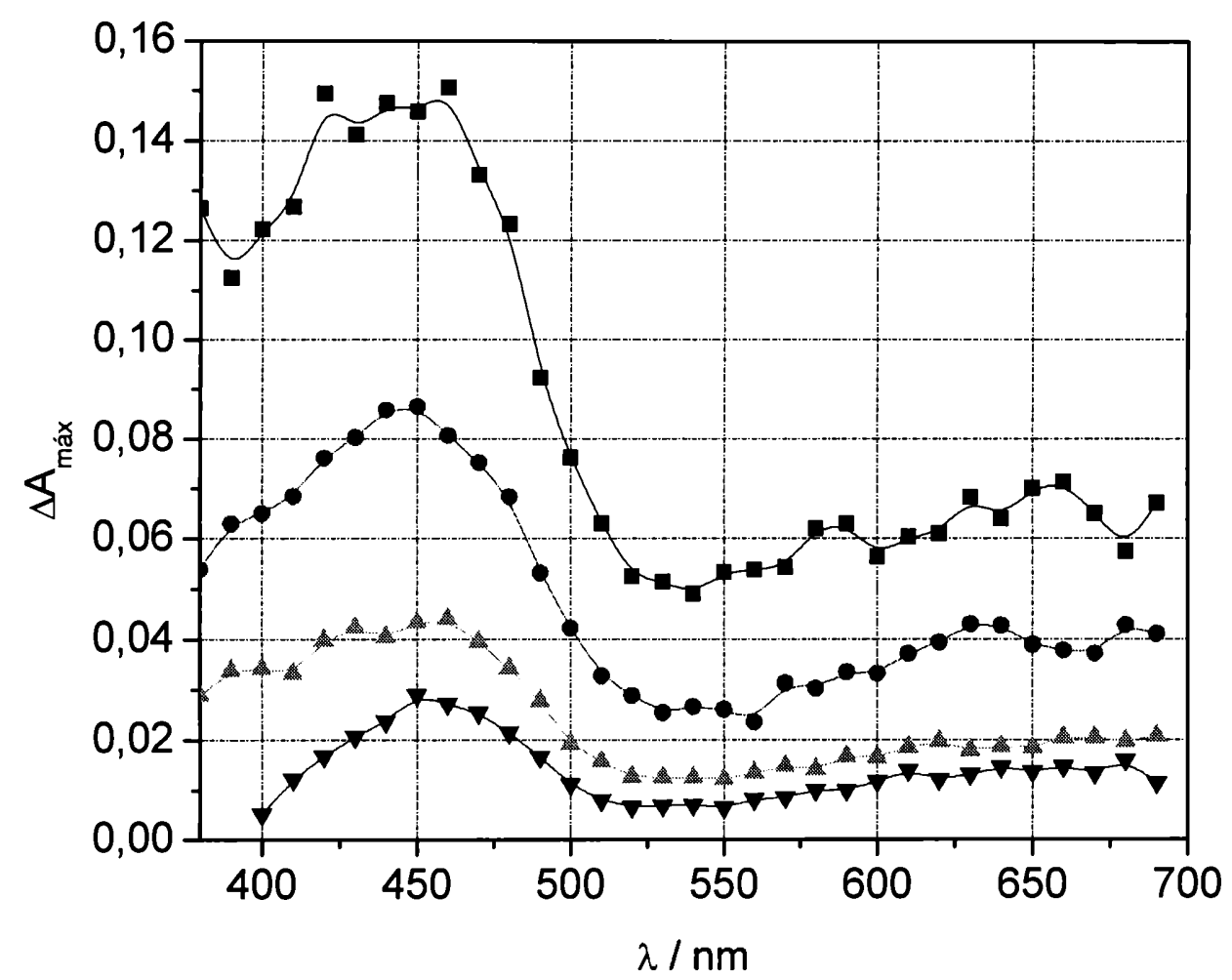

Figura 7: Espectro de absorción del estado excitado TCML de los pendientes

$-\operatorname{Re}(\mathrm{CO})_{3}(\mathrm{tmphen})^{+}$en los polímeros $\left\{(\mathrm{vpy})_{2} \mathrm{vpyRe}(\mathrm{CO})_{3}\left(\mathrm{tmephen}^{+}\right\}_{\mathrm{n}-200}(\boldsymbol{m})\right.$, $\left\{(\mathrm{vpy})_{2} \mathrm{vpy} \operatorname{Re}(\mathrm{CO})_{3}(\text { tmephen })^{+}\right\}_{\mathrm{n} \sim 180}\left\{(\mathrm{vpy})_{2} \mathrm{vpyRe}(\mathrm{CO})_{3}\left(\mathrm{NO}_{2}-\mathrm{phen}\right)^{+}\right\}_{\mathrm{m} \sim 20}(\bullet)$

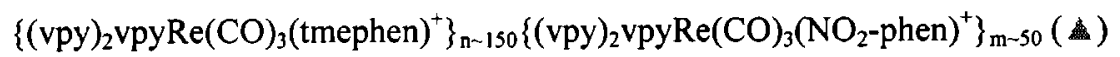
y $\left\{(\text { vpy })_{2} \text { vpyRe }(\mathrm{CO})_{3}(\text { tmephen })^{+}\right)_{n \sim 100}\left\{(\text { vpy })_{2} \text { vpyRe }(\mathrm{CO})_{3}\left(\mathrm{NO}_{2}-\text { phen }\right)^{+}\right\}_{\mathrm{m} \sim 10}(\nabla)$ El espectro fue tomado inmediatamente luego del pulso mostrando $\Delta \mathrm{A}_{\text {máx }}$ vs. $\lambda$. Las soluciones se prepararon en $\mathrm{CH}_{3} \mathrm{CN}$ con concentraciones de cromòforos de $\operatorname{Re}^{\mathrm{I}}$ $\left[\operatorname{Re}^{\mathrm{l}}\right]=5.0 \times 10^{-4} \mathrm{M}$, las soluciones fueron flash irradiadas con $\lambda_{\text {exc }}: 351 \mathrm{~nm}$. 


\begin{tabular}{|l|l|l|}
\hline Polímero & $\tau_{\text {rápido }} / \mathrm{ns}$ & $\mathrm{T}_{\text {lento }} / \mu \mathrm{s}$ \\
\hline$\left\{(\mathrm{vpy})_{2} \operatorname{Re}(\mathrm{CO})_{3}\left(\mathrm{NO}_{2} \text {-phen }\right)^{+}\right\}_{\mathrm{n} \sim 200}$ & $0.23 \pm 0.01$ & \\
\hline$\left\{(\mathrm{vpy})_{2} \mathrm{vpyRe}(\mathrm{CO})_{3}(\text { tmephen })^{+}\right\}_{\mathrm{n} \sim 200}$ & $(7.4 \pm 0.5) 10^{2}$ & $3.4 \pm 0.5$ \\
\hline$\left\{(\mathrm{vpy})_{2} \text { pyRe}(\mathrm{CO})_{3}(\mathrm{tmephen})^{+}\right\}_{\mathrm{n}-180}\left\{(\mathrm{vpy})_{2} \mathrm{vpyRe}(\mathrm{CO})_{3}\left(\mathrm{NO}_{2}-\text { phen }\right)^{+}\right\}_{\mathrm{m} \sim 20}$ & $(1.1 \pm 0.2) \times 10^{2}$ & $1.13 \pm 0.07$ \\
\hline$\left\{(\mathrm{vpy})_{2} \mathrm{vpyRe}(\mathrm{CO})_{3}(\text { tmephen })^{+}\right\}_{\mathrm{n} \sim 150}\left\{(\mathrm{vpy})_{2} \mathrm{vpyRe}(\mathrm{CO})_{3}\left(\mathrm{NO}_{2}-\text { phen }\right)^{+}\right\}_{\mathrm{m} \sim 50}$ & $50 \pm 10$ & $0.59 \pm 0.07$ \\
\hline$\left\{(\mathrm{vpy})_{2} \mathrm{vpyRe}(\mathrm{CO})_{3}(\text { tmephen })^{+}\right)_{\mathrm{n} \sim 100}\left\{(\mathrm{vpy})_{2} \mathrm{vpyRe}(\mathrm{CO})_{3}\left(\mathrm{NO}_{2} \text {-phen }\right)^{+}\right\}_{\mathrm{m} \sim 100}$ & $<10$ & $0.047 \pm 0.006$ \\
\hline
\end{tabular}

Tabla 4: Tiempos de vida de los transientes generados en los polímeros: $\left.\{\text { (vpy })_{2} v p y \operatorname{Re}(\mathrm{CO})_{3}(\text { tmephen })^{+}\right\}_{\mathrm{n}-200}$; $\left\{(\mathrm{vpy})_{2} \operatorname{Re}(\mathrm{CO})_{3}\left(\mathrm{NO}_{2} \text {-phen }\right)^{+}\right\}_{\mathrm{n}-200} ;\left\{(\mathrm{vpy})_{2} \mathrm{vpyRe}(\mathrm{CO})_{3}(\text { tmephen })^{+}\right\}_{\mathrm{n}-180}\left\{(\mathrm{vpy})_{2} \mathrm{vpyRe}(\mathrm{CO})_{3}\left(\mathrm{NO}_{2}-\mathrm{phen}\right)^{+}\right\}_{\mathrm{m}-20} ;$ $\left\{(\text { vpy })_{2} \text { vpyRe }(\mathrm{CO})_{3}(\text { tmepOhen })^{+}\right\}_{\mathrm{n}-150}\left\{(\mathrm{vpy})_{2} \text { vpyRe }(\mathrm{CO})_{3}\left(\mathrm{NO}_{2}-\mathrm{phen}\right)^{+}\right\}_{\mathrm{m}-50} \mathrm{y}$ $\left\{(\text { vpy })_{2} \text { vpyRe }(\mathrm{CO})_{3}(\text { tmephen })^{+}\right)_{\mathrm{n}-100}\left\{(\mathrm{vpy})_{2} \mathrm{vpyRe}(\mathrm{CO})_{3}\left(\mathrm{NO}_{2}-\mathrm{phen}\right)^{+}\right\}_{\mathrm{m} \sim 100}$, con la técnica de láser flash fotólisis

\section{v. Espectroscopía de emisión resuelta en el tiempo}

Se determinó el tiempo de vida de emisión de los polímeros,

$\left\{(\mathrm{vpy})_{2} \mathrm{vpyRe}(\mathrm{CO})_{3}(\text { tmephen })^{+}\right\}_{\mathrm{n} \sim 200}$;

$\left\{(\mathrm{vpy})_{2} \mathrm{vpyRe}(\mathrm{CO})_{3}(\mathrm{tmephen})^{+}\right\}_{\mathrm{n} \sim 180}\left\{(\mathrm{vpy})_{2} \mathrm{vpyRe}(\mathrm{CO})_{3}\left(\mathrm{NO}_{2}-\text { phen }\right)^{+}\right\}_{\mathrm{m} \sim 20} ;$

$\left\{(\text { vpy })_{2} \text { vpyRe }(\mathrm{CO})_{3}(\text { tmephen })^{+}\right\}_{n \sim 150}\left\{(\text { vpy })_{2} \text { vpyRe }(\mathrm{CO})_{3}\left(\mathrm{NO}_{2}-\text { phen }\right)^{+}\right\}_{\mathrm{m} \sim 50} \mathrm{y}$

$\left\{(\text { vpy })_{2} \operatorname{vpyRe}(\mathrm{CO})_{3}(\text { tmephen })^{+}\right)_{\mathbf{n} \sim 100}\left\{(\text { vpy })_{2} \operatorname{vpyRe}(\mathrm{CO})_{3}\left(\mathrm{NO}_{2}-\text { phen }\right)^{+}\right\}_{\mathbf{m} \sim 100}$

a través del análisis de las trazas de emisión resuelta en el tiempo, figura 8, tomadas luego de la irradiación con luz de longitud de onda de $337 \mathrm{~nm}$, proveniente de un láser de $\mathrm{N}_{2}$. El experimento se realizó sobre soluciones preparadas en $\mathrm{CH}_{3} \mathrm{CN}$ deaereadas con $\mathrm{N}_{2}$ ultrapuro y a temperatura ambiente. 
Para el polímero $\left\{(\mathrm{vpy})_{2} \mathrm{vpyRe}(\mathrm{CO})_{3}(\text { tmephen })^{+}\right\}_{n \sim 200}$ el decaimiento de las trazas de emisión fue monoexponencial con un tiempo de vida $\tau_{\mathrm{em}}=5.12 \mu \mathrm{s}$.

Los decaimientos de emisión para los polímeros mixtos fueron no-exponenciales, figura 8, y se ajustaron utilizando la ecuación 33: $N_{t}=N_{0} \exp \left[-\frac{t}{\tau_{D}}-a \sqrt{t / \tau_{D}}\right]$ usando $\tau_{\mathrm{D}}: \quad 5.2 \mu \mathrm{s}$, (tiempo de vida de emisión del polímero $\left\{(\mathrm{vpy})_{2} \mathrm{vpyRe}(\mathrm{CO})_{3}(\text { tmephen })^{+}\right\}_{\mathrm{n} \sim 200}$ en ausencia de quenching). De este ajuste se calcula el valor de $a$. Con este valor, el valor del radio crítico de Förster $R_{\mathrm{F}}: 10.7 \AA$, calculado a partir de la ecuación 26 , y la ecuación 34 se puede calcular $\rho$, la cantidad de sitios quenching por unidad de volúmen. Por último con el valor de $a$ y por medio de la ecuación 35 , se obtiene el valor de $\mathrm{N}$, número de sitios quenching dentro del radio crítico de Förster. Tabla 5.

\begin{tabular}{|c|c|c|c|}
\hline Polímero & $a$ & $\mathrm{~N}$ & $\rho / \mathrm{N}_{\text {aceptores }} / \AA^{3}$ \\
\hline$\left\{(\mathrm{vpy})_{2} \mathrm{vpyRe}(\mathrm{CO})_{3}(\text { tmephen })+\right\}_{n \sim 180}\left\{(\mathrm{vpy})_{2} \mathrm{vpyRe}(\mathrm{CO})_{3}\left(\mathrm{NO}_{2}-\text { phen }\right)+\right\}_{m \sim 20}$ & 1.90 & 1.1 & $2.1 \times 10^{-4}$ \\
\hline$\left\{(\mathrm{VPy})_{2} \mathrm{vpyRe}(\mathrm{CO})_{3}(\mathrm{tmephen})+\right\}_{n \sim 150}\left\{(\mathrm{vpy})_{2} \mathrm{vpyRe}(\mathrm{CO})_{3}\left(\mathrm{NO}_{2}-\mathrm{phen}\right)+\right\}_{\mathrm{m} \sim 50}$ & 4.6 & 2.6 & $5.05 \times 10^{-4}$ \\
\hline$\left\{(\mathrm{vpy})_{2} \mathrm{vpyRe}(\mathrm{CO})_{3}(\text { tmephen })+\right)_{n \sim 100}(\mathrm{vpy})_{2} \mathrm{vpyRe}(\mathrm{CO})_{3}\left(\mathrm{NO}_{2}-\right.$ phen $\left.)+\right\}_{m-100}$ & 19.3 & 11 & $2.1 \times 10^{-3}$ \\
\hline
\end{tabular}

Tabla 5: Valores calculados de $a, \mathrm{~N}$ y $\rho$ por análisis de las trazas de emisión resuelta en el tiempo de los polímeros mixtos. 

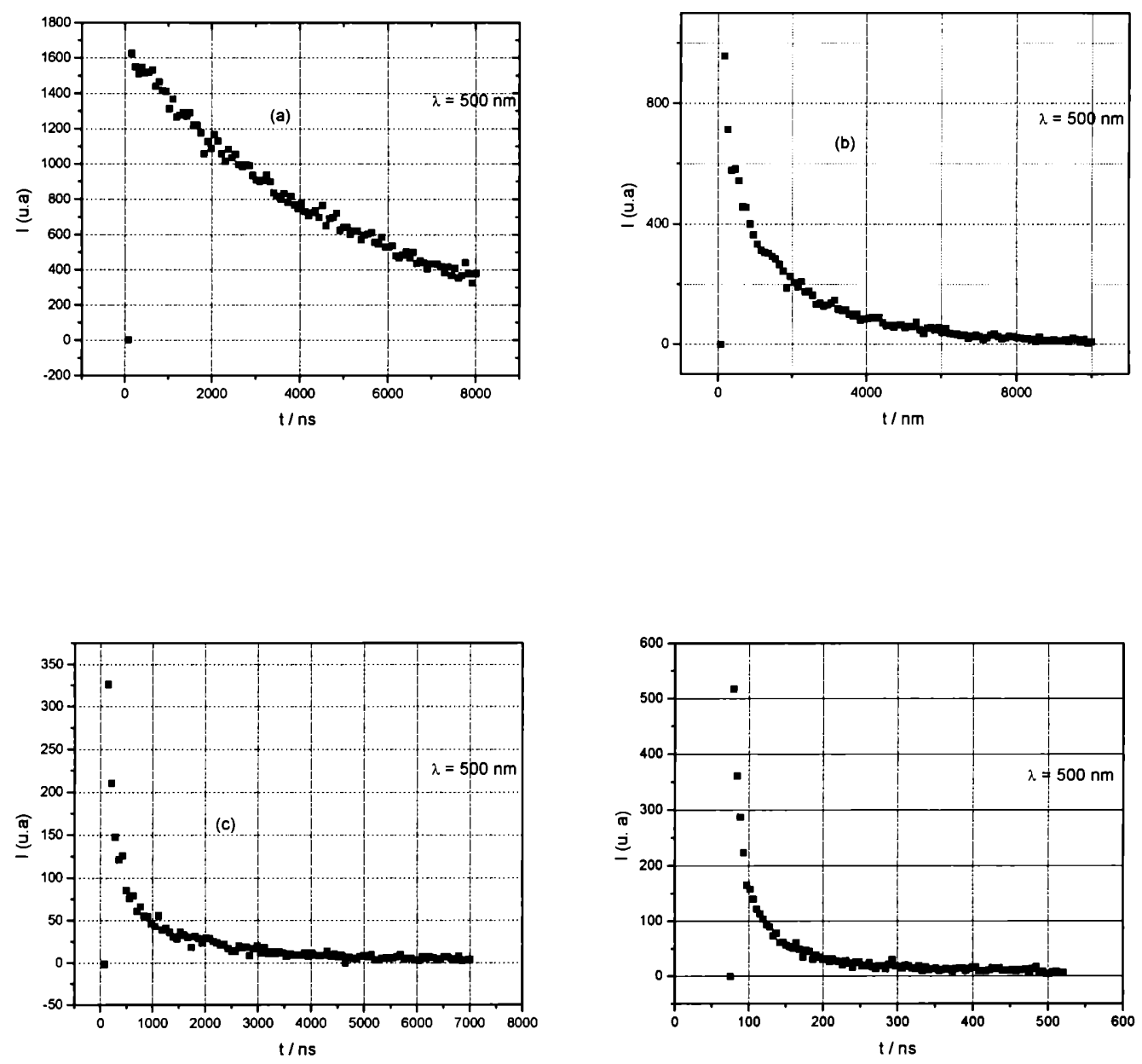

Figura 8: Trazas de los decaimientos de emisión resuelta en el tiempo de los polímeros a: $\left\{(\mathrm{vpy})_{2} \mathrm{vpyRe}(\mathrm{CO})_{3}(\operatorname{tmephen})^{+}\right\}_{n \sim 200}$; b: $\left.\left\{(\mathrm{vpy})_{2} \mathrm{vpyRe}(\mathrm{CO})_{3} \text { (tmephen }\right)^{+}\right\}_{\mathrm{n} \sim 180}\left\{(\mathrm{vpy})_{2} \mathrm{vpy} \operatorname{Re}(\mathrm{CO})_{3}\left(\mathrm{NO}_{2} \text {-phen }\right)^{+}\right\}_{\mathrm{m}-20} ;$ c: $\left\{(\text { vpy })_{2} \text { vpyRe }(\mathrm{CO})_{3}(\text { tmephen })^{+}\right\}_{\mathrm{n}-150}\left\{(\mathrm{vpy})_{2} \mathrm{vpyRe}(\mathrm{CO})_{3}\left(\mathrm{NO}_{2}-\mathrm{phen}\right)^{+}\right\}_{\mathrm{m} \sim 50} \mathrm{y}$ $\mathrm{d}\left\{(\mathrm{vpy})_{2} \mathrm{vpyRe}(\mathrm{CO})_{3}(\text { tmephen })^{+}\right)_{\mathrm{n} \sim 100}\left\{(\mathrm{vpy})_{2} \mathrm{vpyRe}(\mathrm{CO})_{3}\left(\mathrm{NO}_{2} \text {-phen }\right)^{+}\right\}_{\mathrm{m} \sim 100}$ registrados con un láser excímero $\mathrm{XeF}$ de $\lambda_{\text {exc }}: 351 \mathrm{~nm}$ sobre soluciones preparadas en acetonitrilo y deaereadas con $\mathrm{N}_{2}$ ultrapuro a $T$ ambiente 


\section{Discusión y Conclusiones}

Para el desarrollo de la discusión y conclusiones se identificará a los polímeros con la siguiente numeración:

$$
\begin{aligned}
& \left\{(\text { vpy })_{2} \operatorname{vpyRe}(\mathrm{CO})_{3}(\text { tmephen })^{+}\right\}_{n \sim 200}=\mathrm{I} \\
& \left\{(\mathrm{vpy})_{2} \operatorname{Re}(\mathrm{CO})_{3}\left(\mathrm{NO}_{2} \text {-phen }\right)^{+}\right\}_{\mathrm{n} \sim 200}=\mathrm{II} \\
& \left\{(\text { vpy })_{2} \text { vpyRe }(\mathrm{CO})_{3}(\text { tmephen })^{+}\right\}_{\mathrm{n} \sim 180}\left\{(\mathrm{vpy})_{2} \mathrm{vpyRe}(\mathrm{CO})_{3}\left(\mathrm{NO}_{2}-\text { phen }\right)^{+}\right\}_{\mathrm{m} \sim 20}=\text { III } \\
& \left\{(\mathrm{vpy})_{2} \mathrm{vpyRe}(\mathrm{CO})_{3}(\text { tmephen })^{+}\right\}_{\mathrm{n} \sim 150}\left\{(\mathrm{vpy})_{2} \operatorname{vpyRe}(\mathrm{CO})_{3}\left(\mathrm{NO}_{2}-\mathrm{phen}\right)^{+}\right\}_{\mathrm{m} \sim 50}=\mathrm{IV} \\
& \left.\left\{(\mathrm{vpy})_{2} \mathrm{vpyRe}(\mathrm{CO})_{3}(\text { tmephen })^{+}\right)_{\mathrm{n} \sim 100}\left\{(\mathrm{vpy})_{2} \mathrm{vpyRe}(\mathrm{CO})_{3}\left(\mathrm{NO}_{2}-\mathrm{phen}\right)^{+}\right\}_{\mathrm{m} \sim 100}=\mathrm{V}\right\}
\end{aligned}
$$

\section{i. Espectroscopia de emisión estacionaria}

Del análisis de los rendimientos cuánticos de emisión se puede concluir en primer lugar, que en el polímero I, una de las vías de desactivación para el estado excitado de $\mathrm{TCML}_{\mathrm{Re} \rightarrow \text { tmephen }}$ es la emisión de luz, luminiscencia, mientras que el polímero II no presenta

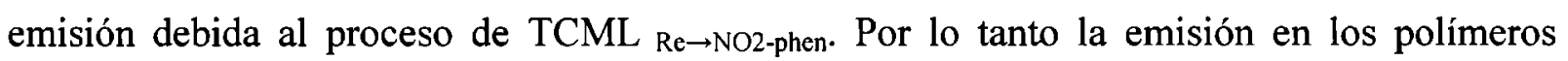
mixtos; III, IV y V se debe sólo a la contribución del cromóforo - $\operatorname{Re}(\mathrm{CO})_{3}(\text { tmephen })^{+}$.

En segundo lugar, analizando los rendimientos cuánticos de emisión obtenidos para los polímeros mixtos (tabla 2) puede inferirse que la sustitución de cromóforos $-\operatorname{Re}(\mathrm{CO})_{3}(\text { tmephen })^{+}$por cromóforos $-\operatorname{Re}(\mathrm{CO})_{3}\left(\mathrm{NO}_{2} \text {-phen }\right)^{+}$en la poli-4-vinilpiridina produce una disminución del $\phi_{\mathrm{em}}$ mayor que la esperada por la disminución de cromóforos emisores, $-\operatorname{Re}(\mathrm{CO})_{3}(\text { tmephen })^{+}$. 
De acuerdo con estos resultados experimentales, es posible concluir que en los polímeros mixtos ocurre un proceso de desactivación que involucra al estado excitado $\mathrm{TCML}_{\mathrm{Re} \rightarrow \text { mphen }} \mathrm{y}$ al cromóforo $-\mathrm{Re}(\mathrm{CO})_{3}\left(\mathrm{NO}_{2} \text {-phen }\right)^{+}$como aceptor.

La desactivación, que se traduce en un quenching de la luminiscencia, ocurre cuando ambos cromóforos se encuentran dentro de la misma cadena de polímero, ya que los rendimientos cuánticos de emisión en las soluciones mezcla de polímeros, determinados experimentalmente (tabla 3), concuerdan perfectamente con los calculados a partir de la expresión. $\phi_{e m}=\left(\frac{A}{A_{T}}\right) \phi_{e m}^{0}$ que es la expresión del rendimiento cuántico de emisión de los polímeros mixtos, si el quenching no hubiera ocurrido.

Además, los decaimientos de la absorción de los transientes generados en los polímeros mixtos (figura 6) no evidencian la generación de fotoproductos y/o intermediarios, como por ejemplo formación de $\operatorname{Re}(\mathrm{CO})_{3}(\mathrm{tmphen})^{+2}$ y $\operatorname{Re}(\mathrm{CO})_{3}\left(\mathrm{NO}_{2} \text {-phen }\right)^{\bullet}$, por lo tanto, se puede concluir que la desactivación del cromóforo $-\operatorname{Re}(\mathrm{CO})_{3}(\mathrm{tmphen})^{+}$en los polímeros mixtos se produce por transferencia de energía al cromóforo aceptor $-\operatorname{Re}(\mathrm{CO})_{3}\left(\mathrm{NO}_{2}\right.$-phen y no por transferencia de carga.

Esta transferencia de energía es posible, debido a que como ya se discutió en el capítulo anterior, al analizar el espectro UV-Visible, puede observarse que el estado de $\mathrm{TCML}_{\mathrm{Re} \rightarrow \text { tmphen }}$ se genera a mayor energía que el estado excitado de $\mathrm{TCML}_{\mathrm{Re} \rightarrow \mathrm{NO2} \text {-phen }}$.

De acuerdo a estos resultados, se puede considerar a los polímeros mixtos estudiados, como polímeros derivatizados con donores de energía $-\operatorname{Re}(\mathrm{CO})_{3} \operatorname{tmephen~}^{+} \mathrm{y}$ aceptores $-\operatorname{Re}(\mathrm{CO})_{3} 5-\mathrm{NO}_{2}-\mathrm{phen}^{+}$, distribuidos aleatoriamente a lo largo del esqueleto de la poli4vinilpiridina, $(\text { vpy })_{600}$. 
Localmente, donor y aceptor pueden poseer regiones sin piridinas espaciadoras (D-A, D-D, A-A), una piridina espaciadora (D-py-A, D-py-D, A-py-A) y dos o mas piridinas espaciadoras (D-py-py-D, D-py-py-A) etc.

Los tiempos de vida de los estados excitados de transferencia de carga metal ligando y la dinámica de la transferencia de carga son dependientes del ambiente local, por lo que hay una distribución de sitios sobre cada cadena de polímero individual.

De acuerdo a un cálculo de modelado molecular, ver mas adelante, en promedio la distancia periférica entre vecinos mas cercanos debe ser $7 \AA$. A distancias entre vecinos de $7 \AA$ o menores, el proceso de transferencia de energía puede ser posible por medio del mecanismo de Förster, Dexter o una combinación de ambos. El valor de $R_{F}: 10.7 \AA$, se encuentra en el límite inferior de los valores de $R_{F}$ (típicamente entre 10 y $70 \AA$ ) esto es debido al pobre solapamiento entre el espectro de emisión del donor y el espectro de absorción del aceptor.

Con el fin de dilucidar la naturaleza del proceso de transferencia de energía intramolecular en el sistema de polímeros mixtos, se realizó un experimento de quenching de luminiscencia del polimero I, usando $\mathrm{ClRe}(\mathrm{CO})_{3} 5-\mathrm{NO}_{2}$-phen como aceptor de energía en una mezcla de solventes glicerol: etanol: diclorometano (GED), medio en el cual la difusión se encuentra restringida, además se realizó el mismo experimento en acetonitrilo como solvente (figura 9). La mezcla de solventes presenta una viscosidad $\eta$ : 0.23 poise, calculada a T: $25{ }^{0} \mathrm{C}$ con un viscosímetro modelo Rotovisco HAAKE RV2. Con el dato de viscosidad puede ser calculada una constante de difusión por medio de la siguiente expresión:

$$
k_{\text {dif }}=\frac{2 \times 10^{5} T}{\eta}
$$


donde $\mathrm{T}$ es la temperatura en ${ }^{\circ} \mathrm{K}$ y, la viscosidad expresada en poise. El valor calculado dio $\mathrm{k}_{\text {dif: }}: 2.0 \times 10^{8} \mathrm{M}^{-1} \mathrm{~s}^{-1}$

En el solvente GED, el quenching es observado a concentraciones de $\mathrm{ClRe}(\mathrm{CO})_{3} 5-\mathrm{NO}_{2}$-phen tan bajas como $2 \times 10^{-4} \mathrm{M}$, con la cual puede calcularse un tiempo de difusión

$$
\tau_{\mathrm{dif}}=1 / \mathrm{k}_{\mathrm{dif}} \times[\mathrm{Q}]=25 \mu \mathrm{s}
$$

Dicho $\tau_{\text {dif }}$ es aproximadamente 5 veces más grande que el tiempo de vida de luminiscencia del polímero $\left\{(\mathrm{vpy})_{2} \mathrm{vpyRe}(\mathrm{CO})_{3}(\text { tmephen })^{+}\right\}_{\mathrm{n} \sim 200}(\tau: 5.2 \mu \mathrm{s})$.
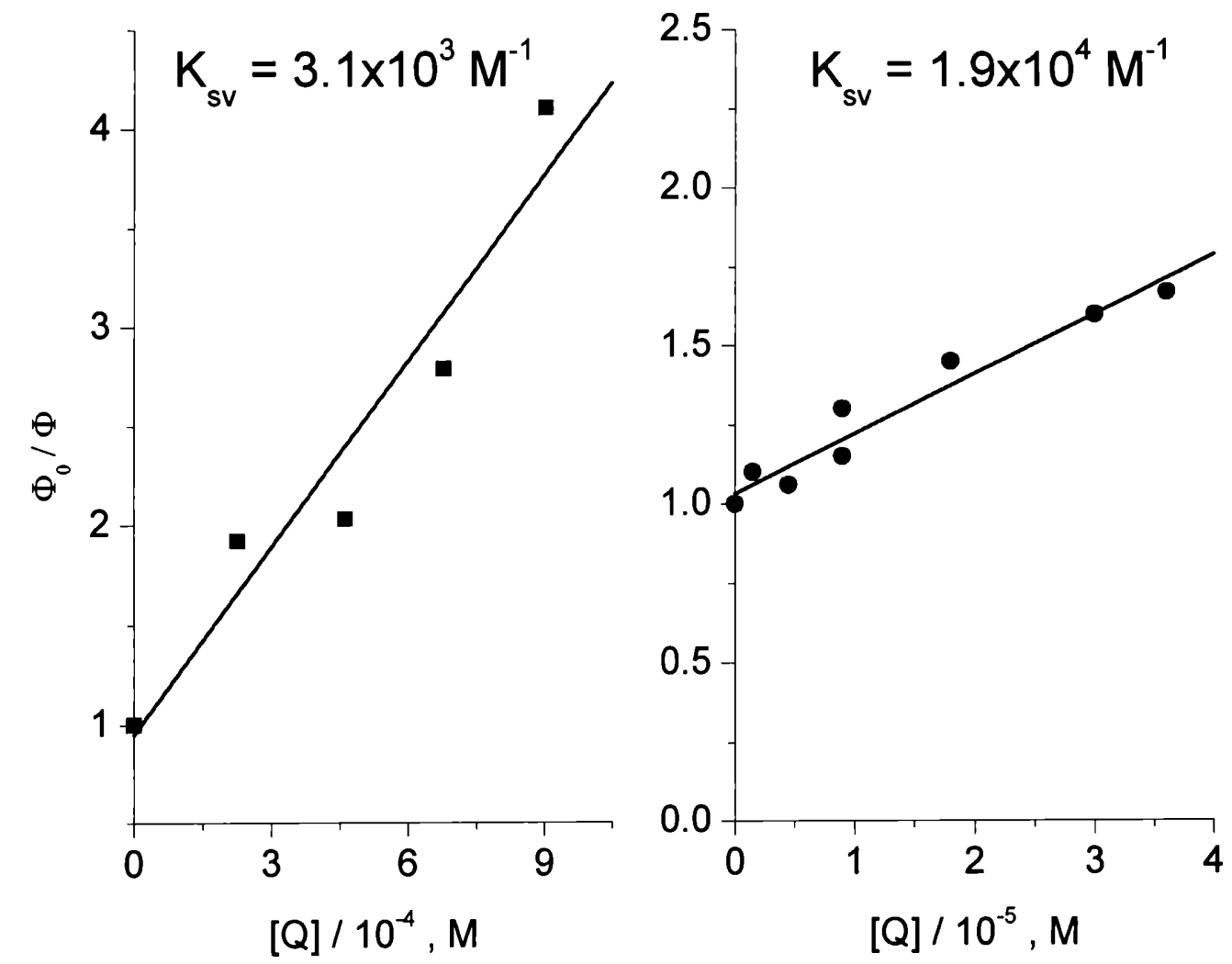

Figura 9: Gráfico de Stern-Volmer del quenching de luminiscencia de $\left\{(\mathrm{vpy})_{2} \operatorname{vpyRe}(\mathrm{CO})_{3}(\operatorname{tmephen})^{+}\right\}_{\mathrm{n} \sim 200} \operatorname{con}$ $\mathrm{CIRe}(\mathrm{CO})_{3}\left(\mathrm{NO}_{2}\right.$-phen) en acetonitrilo $(\bullet)$ y en $\operatorname{GED}(\boldsymbol{\bullet})$. 
A una concentración de $\left(\mathrm{ClRe}(\mathrm{CO})_{3}\left(5-\mathrm{NO}_{2}\right.\right.$-phen)) de $2 \times 10^{-4} \mathrm{M}$ (en GED) la distancia promedio entre moléculas donor y aceptor es de aproximadamente $110 \AA^{12}$ (figura 2), por lo tanto durante el tiempo de vida de emisión del polímero las moléculas de $\left(\mathrm{ClRe}(\mathrm{CO})_{3}\left(5-\mathrm{NO}_{2}\right.\right.$ phen)) podrán difundir 20-30 $\AA$ y en promedio estarán "lejos" del centro luminiscente (d > 70 $\AA)$. A esta gran separación entre donor y aceptor el mecanismo de Förster para la transferencia de energía se ve desfavorecido con respecto al mecanismo de Dexter.

Con el fin de tener una mejor apreciación del efecto estructural y características morfológicas de los polímeros en las propiedades fotofísicas, se realizó un modelado molecular sobre el polímero I, dicho cálculo fue realizado usando Hyperchem 7 utilizando el mecanismo de optimización molecular con un algoritmo Polak-Ribieri (gradiente conjugado) basado en un polímero atáctico con pendientes $\operatorname{Re}(\mathrm{CO})_{3} \mathrm{tmphen}^{+}$similar al polímero I. Para el cálculo el polímero construido contiene $24 \mathrm{Re}(\mathrm{CO})_{3}$ tmephen $^{+}$coordinando $1 / 3$ de un total de 72 piridinas.

Esto representa alrededor del $12 \%$ del polímero (donde hay $200 \mathrm{Re}(\mathrm{CO})_{3} \operatorname{tmphen}^{+}$ pendientes coordinando $1 / 3$ de 600 piridinas presentes por molécula de polímero). No fueron incluidas las moléculas de solvente en este cálculo y sólo es una representación muy aproximada de la molécula real. Sin embargo puede ayudar a visualizar la distribución de distancias entre centros $\mathrm{Re}^{\mathrm{I}}$. Los 24 átomos de $\mathrm{Re}(\mathrm{I})$ de la figura 10 fueron numerados como

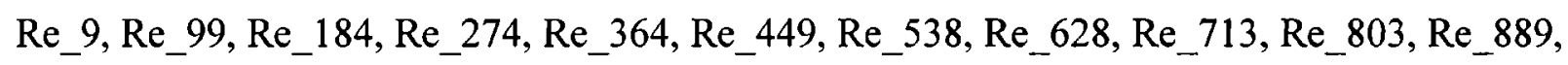
Re_974, Re_1066, Re_1156, Re_1241, Re_1331, Re_1421, Re_1506, Re_1595, Re_1685, Re_1770, Re_1860, Re_1946, y Re_2031 en la tabla 6. 


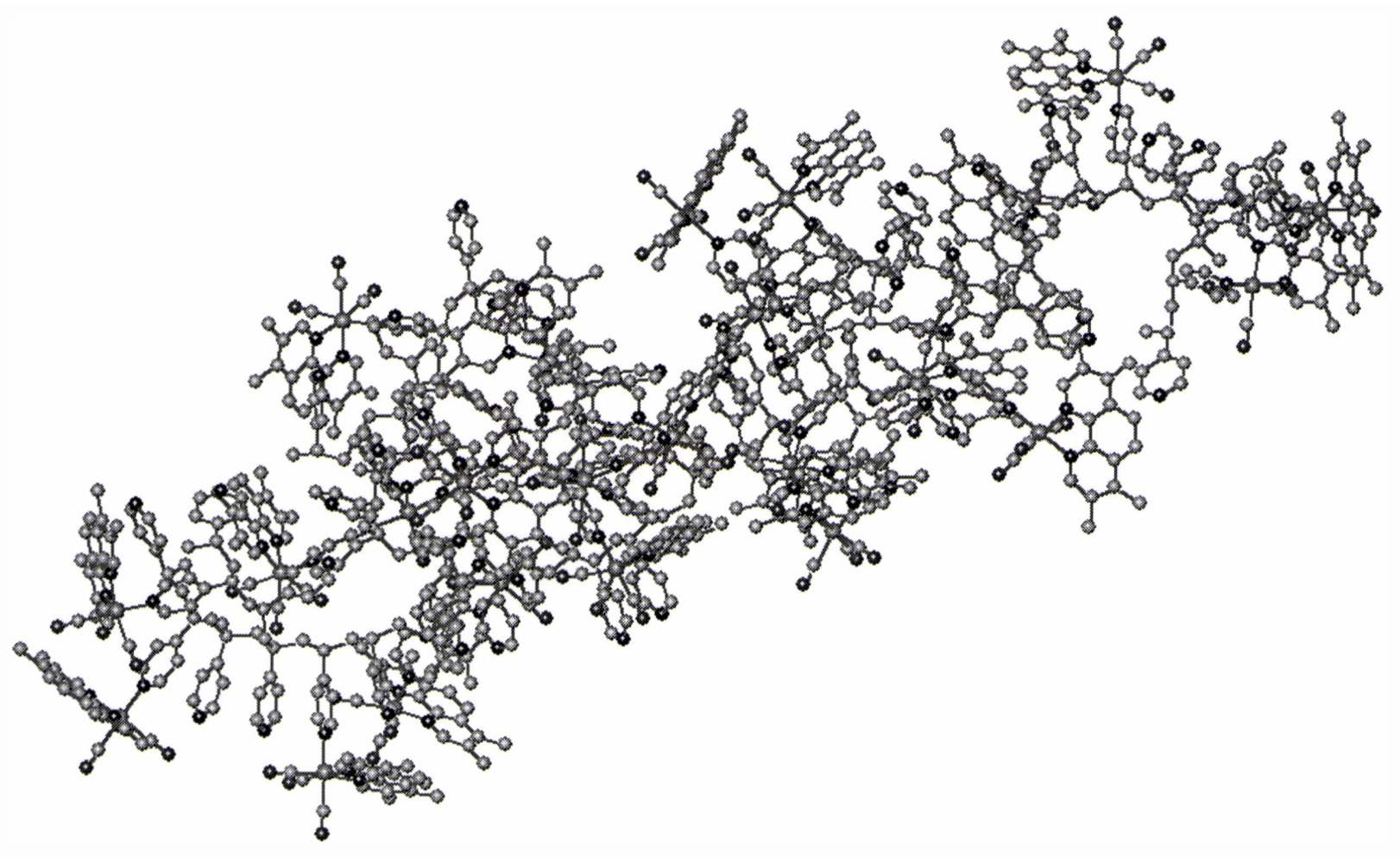

Figura 10: Representación del polímero I, con $24 \operatorname{Re}(\mathrm{CO})_{3}$ tmephen $^{+}$coordinando $1 / 3$ de un total de 72 piridinas. 


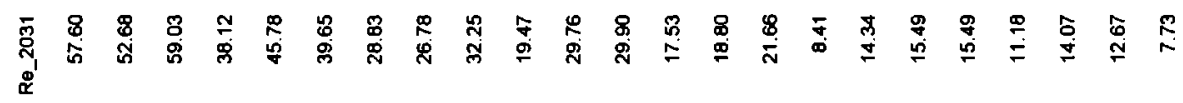

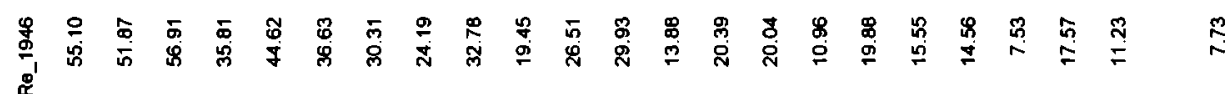

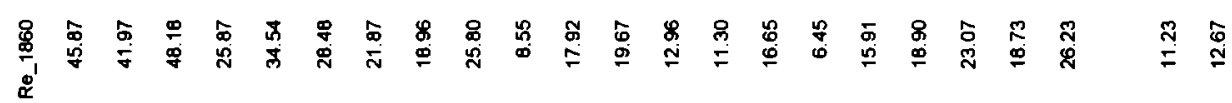

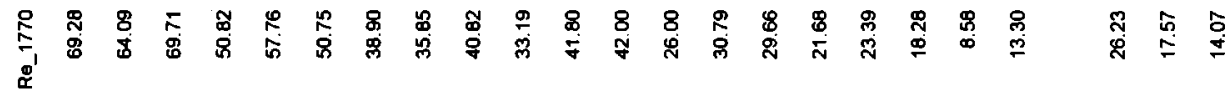

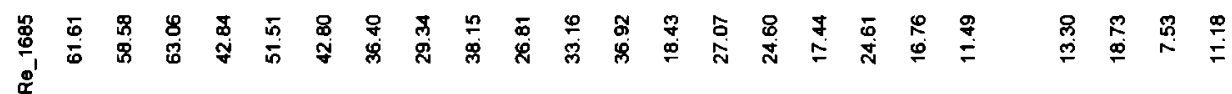

尊兽

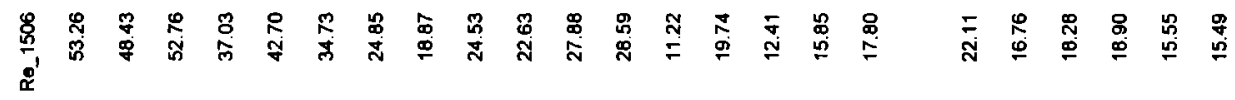

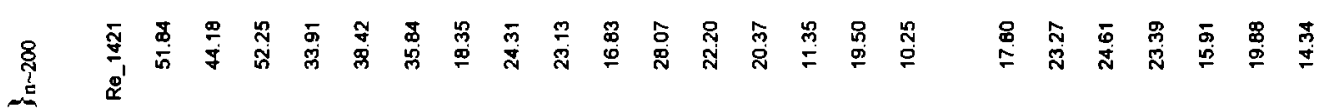

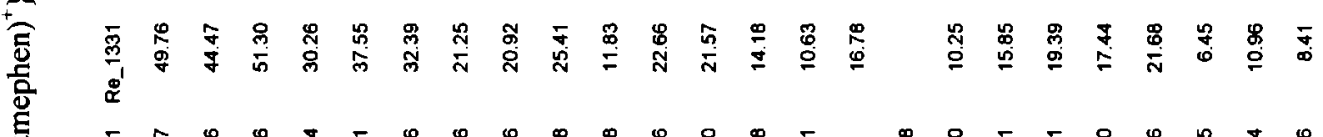

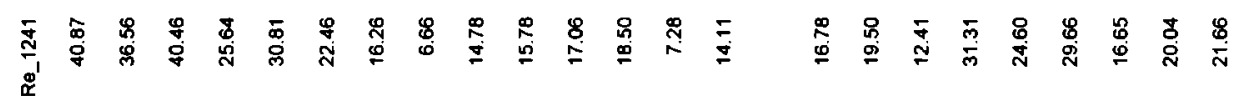

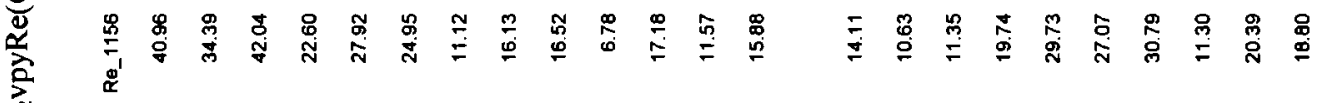

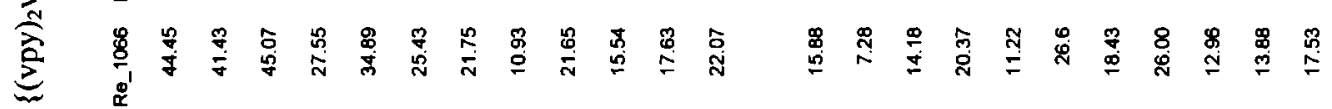
兽 产 $\quad$ 兽

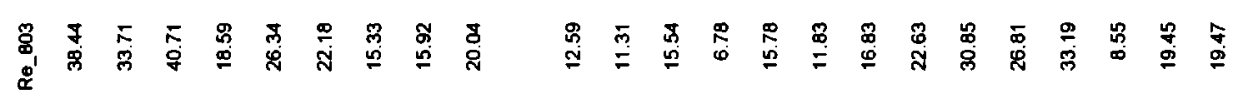

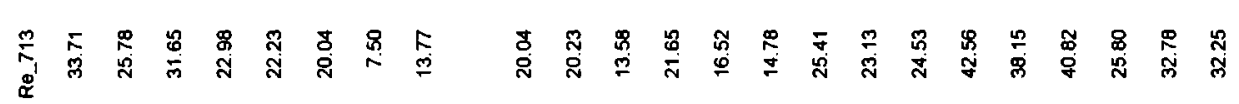

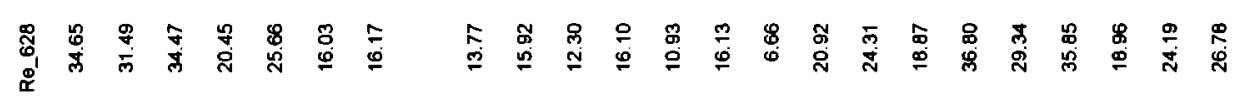

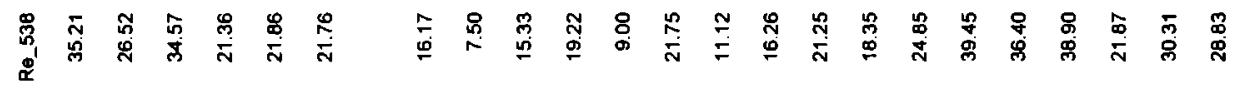

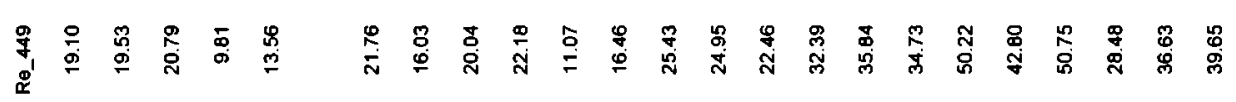

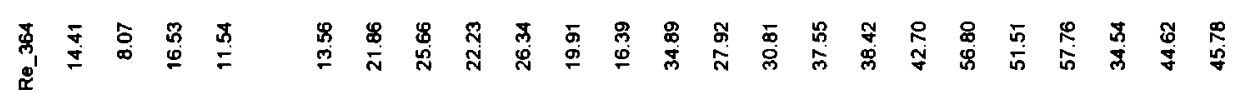

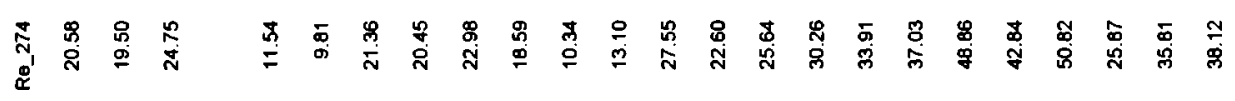

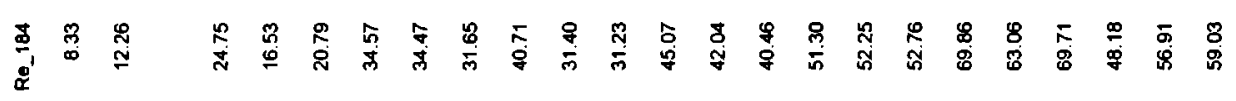

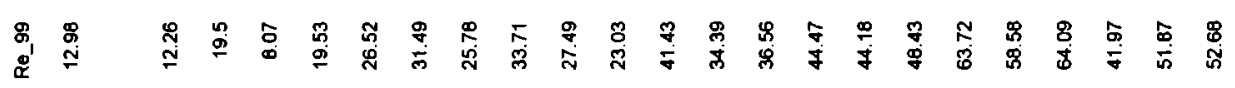

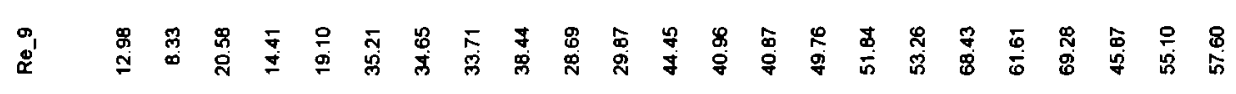

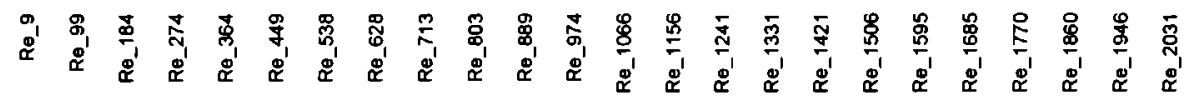


Las distancias Re-Re de la tabla 6 espaciadas entre 6.50 y $72.0 \AA$, fueron usadas para construir un histograma como sigue; el intervalo de distancia total (6.5-72.0 $\AA$ ) fue dividido en 22 intervalos de distancia con $\Delta \mathrm{R}=3 \AA$ (estos intervalos fueron 6-9, 9-12, $12-15,15-18,18-21,21-24,24-27,27-30,30-33,33-36,36-39,39-42,42-45,45-48,48-$ $51,51-54,54-57,57-60,60-63,63-66,66-69$ y $69-72 \AA$, respectivamente) y todas las distancias de la tabla 6 fueron agrupadas dentro de estos 22 intervalos. Este histograma fue graficado en la figura 11 como el número relativo de sitios quenching vs $\mathrm{R}$ ( $\mathrm{R}$ es el centro de cada uno de los 22 intervalos, esto es. 7.5, 10.5, 13.5, 16.5, 19.5, 22.5, 25.5, $28.5,31.5,34.5,37.5,40.5,43.5,46.5,49.5,52.5,55.5,58.5,61.5,64.5,67.5$ y $70.5 \AA$, respectivamente). Este histograma se muestra en la tabla 7.

\begin{tabular}{cccc}
\hline$\Delta R$ & $R / \AA$ & $N$ & $N / N_{\text {total }}$ \\
\hline $6-9$ & 7.5 & 12 & 0.04348 \\
$9-12$ & 10.5 & 19 & 0.06884 \\
$12-15$ & 13.5 & 21 & 0.07609 \\
$15-18$ & 16.5 & 31 & 0.11232 \\
$18-21$ & 19.5 & 32 & 0.11594 \\
$21-24$ & 22.5 & 25 & 0.09058 \\
$24-27$ & 25.5 & 23 & 0.08333 \\
$27-30$ & 28.5 & 17 & 0.06159 \\
$30-33$ & 31.5 & 13 & 0.0471 \\
$33-36$ & 34.5 & 16 & 0.05797 \\
$36-39$ & 37.5 & 12 & 0.04348 \\
$39-42$ & 40.5 & 12 & 0.04348 \\
$42-45$ & 43.5 & 10 & 0.03623 \\
$45-48$ & 46.5 & 3 & 0.01087 \\
$48-51$ & 49.5 & 7 & 0.02536 \\
$51-54$ & 52.5 & 8 & 0.02899 \\
$54-57$ & 55.5 & 3 & 0.01087 \\
$57-60$ & 58.5 & 4 & 0.01449 \\
$60-63$ & 61.5 & 1 & 0.00362 \\
$63-66$ & 64.5 & 3 & 0.01087 \\
$66-69$ & 67.5 & 1 & 0.00362 \\
$69-72$ & 70.5 & 3 & 0.01087
\end{tabular}

Se construyó un mapa de distancias (tomando todas la posibilidades entre 24 centros de $\operatorname{Re}^{\mathrm{I}}$ en el modelo del polímero. La figura 11 muestra la probabilidad de encontrar pendientes de $\operatorname{Re}^{\mathrm{I}}$ a distancia d(Re-Re) desde el pendiente de referencia $\operatorname{Re}^{\mathrm{I}}$ vs 
$\mathrm{d}(\mathrm{Re}-\mathrm{Re})$. La curva representa una gaussiana la cual ha sido truncada a $\mathrm{d}$ : $7 \AA$ (debido a que es físicamente imposible tener $\mathrm{Re}^{\mathrm{I}}$ pendientes a distancias menores que esta)

El máximo de la distribución, el cual puede visualizarse como la distancia promedio Re-Re ocurre a $20 \AA$ y contribuye con el $12 \%$ de los casos. Distancias Re-Re $>30 \AA$ contribuyen con menos del $4 \%$ del total de distancias posibles. El número de vecinos a distancias similares a $R_{F}$ es entre 7 y $8 \%$ del total. Se supone que la distribución en los polímeros mixtos es similar que en el polímero I dado que ambos cromóforos $-\operatorname{Re}(\mathrm{CO})_{3}(\text { tmephen })^{+}$y $-\operatorname{Re}(\mathrm{CO})_{3}\left(5 \mathrm{NO}_{2} \text {-phen }\right)^{+}$tienen volúmenes similares.

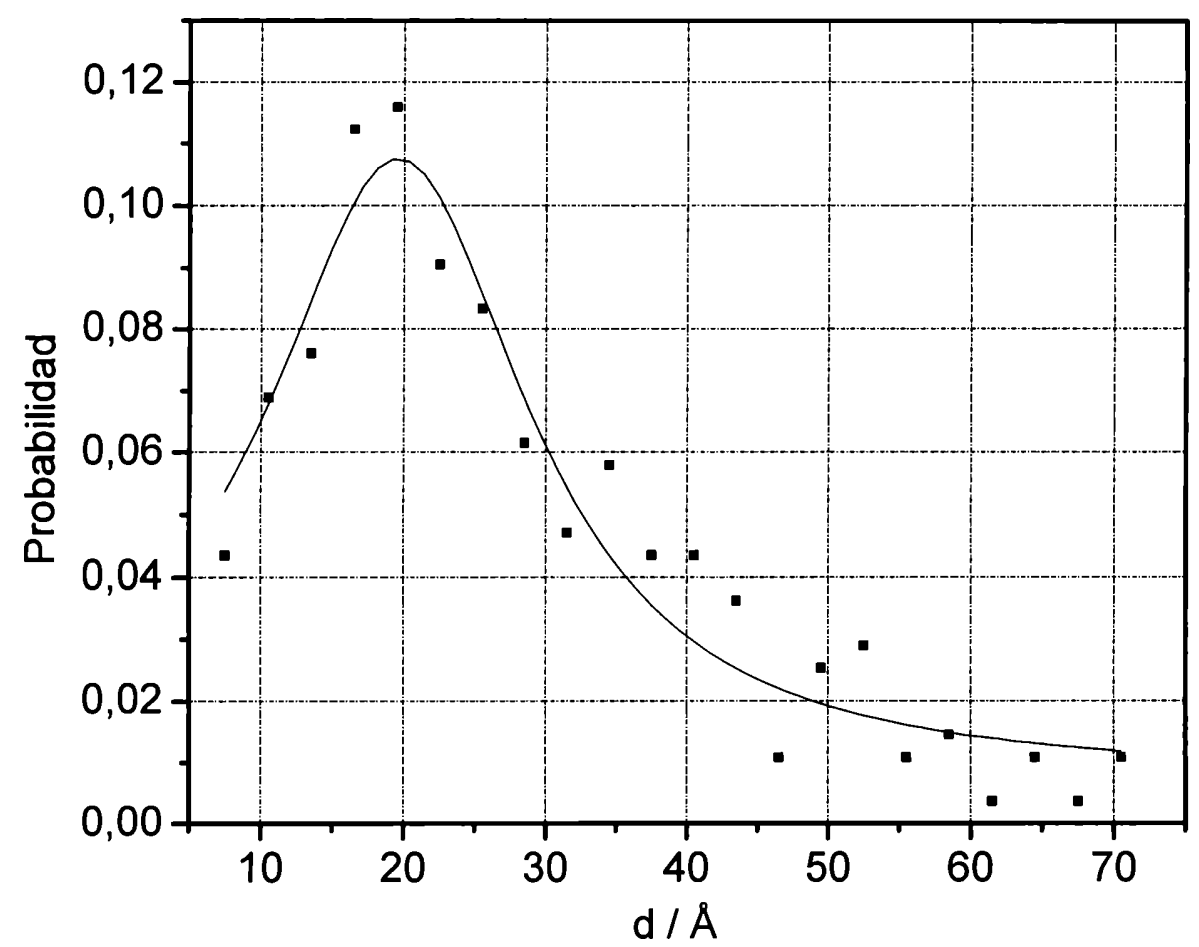

Figura 11: Distribución de distancias entre los centros $\mathrm{Re}^{\mathrm{I}}$ en el polímero $\left\{\left[(\mathrm{vpy})_{2} \mathrm{vpyRe}(\mathrm{CO})_{3}(\text { tmephen })^{+}\right]\right\}_{\mathrm{n} \sim 200}$.

El número de sitios quenching dentro del radio crítico calculado, N (Tabla 5) puede ser comparado con el obtenido desde la distribución de distancias. En los 
polímeros mixtos, III; IV y V, el número de sitios quenching, $-\operatorname{Re}(\mathrm{CO})_{3}\left(\mathrm{NO}_{2} \text {-phen }\right)^{+}$es: 2050 y 100 por molécula respectivamente. Multiplicando este número por la probabilidad de 0.075 (que es la probabilidad de que se encuentren dentro del radio de Förster) se obtiene $\mathrm{N}_{\mathrm{III}}=1.5 ; \mathrm{N}_{\mathrm{IV}}=4$ y $\mathrm{N}_{\mathrm{V}}=7.5$ respectivamente.

En el proceso de transferencia de energía el punto crucial es que cuando se pasa del polímero III al V la probabilidad de que el donor se encuentre en las proximidades del aceptor aumenta. Los donores situados a distancias $\sim 7 \AA$ de un aceptor serán desactivados instantáneamente con un valor de $k_{\mathrm{T}} \approx 13 / \tau_{\mathrm{D}}$ ya que $\mathrm{d}(\mathrm{Re}-\mathrm{Re})<\mathrm{R}_{\mathrm{F}}(10.7$ $\AA$ ) Los donores situados a distancias $\approx 20 \AA$ serán desactivados con un valor de $k_{\mathrm{T}}$ de sólo $0.02 / \tau_{D}$ y contribuirán menos al quenching. Sin embargo el número de aceptores a $\mathrm{d} \approx 20 \AA$ es tres veces mayor que a $\mathrm{d} \approx 7 \AA$.

La eficiencia de transferencia en los polímeros puede ser calculada como

$$
E_{T}^{j}=1-\frac{\phi_{D}^{j}}{\phi_{m m}}
$$

donde $E_{J}$ representa la eficiencia en la transferencia de energía en el polímero

$\mathrm{j}=\mathrm{III} ; \mathrm{IV}$ y V

$\phi_{\mathrm{D}}^{\mathrm{J}}$ es el rendimiento cuántico del polímero mixto $\mathrm{j}, \mathrm{y} \phi_{\mathrm{mm}}$ representa el rendimiento cuántico de emisión de las mezclas molares:

$90 \%$ I $10 \%$ II;

$75 \%$ I $25 \%$ II;

$50 \%$ I $50 \%$ II

El valor de $E_{T}^{J}$ para los polímeros mixtos se muestra en la Tabla 7. Con estos datos de $E_{T}^{J}$ es posible calcular un valor medio de la constante de de transferencia de 
energía entre el estado excitado $\mathrm{TCML}_{\mathrm{Re} \rightarrow \text { mphen }} \mathrm{y}$ el cromóforo $\mathrm{Re}(\mathrm{CO})_{3}\left(5-\mathrm{NO}_{2}\right.$-phen $)$ como

$$
\overline{k_{E T}}=\frac{1}{\tau_{D}}\left(\frac{E_{T}^{j}}{1-E_{T}^{j}}\right)
$$

El valor de $\overline{k_{E T}}$ es sólo una contribución promedio en el polímero (tabla 7). En la evaluación de las $\mathrm{k}_{\mathrm{ET}}$ se debe asumir que el quenching del donor es producido por interacción con regiones vecinas del polímero que contienen el aceptor. La influencia de estas interacciones depende de la distancia entre D y A, por lo tanto la velocidad de quenching para el i-ésimo donor es la suma de las distancias de interacción con $\mathrm{J}$ sitios quenching y $\overline{k_{E T}}$ representa un valor medio de todos los posibles $k_{\mathrm{ET}}(\mathrm{Di})$

$$
k_{E T}\left(D_{i}\right)=\frac{1}{\tau_{D}} \sum_{j}\left(R_{F} / R\left(D_{i}, j\right)\right)^{6}
$$

Usando los valores $E_{T}^{j}$ para los polímeros mixtos, las distancias intercromóforos promedio pueden ser calculados por:

$$
\bar{R}=R_{F}\left(\frac{1}{E_{T}^{j}}-1\right)^{1 / 6}
$$

El valor de $\bar{R}$ se puede observar en la tabla 8 . 


\begin{tabular}{|l|l|l|l|}
\hline Polímero mixto & $E_{J}$ & $k_{\mathrm{ET}}$ & $\bar{R}$ \\
\hline$\left\{(\mathrm{vpy})_{2} \mathrm{vpyRe}(\mathrm{CO})_{3}(\text { tmephen })^{+}\right\}_{\mathrm{n}-180}\left\{(\mathrm{vpy})_{2} \mathrm{vpyRe}(\mathrm{CO})_{3}\left(\mathrm{NO}_{2} \text {-phen }\right)^{+}\right\}_{\mathrm{m} \sim 20}$ & 0.54 & $2.7 \times 10^{5}$ & 10.1 \\
\hline$\left\{(\mathrm{vpy})_{2} \mathrm{vpyRe}(\mathrm{CO})_{3}(\text { tmephen })^{+}\right\}_{\mathrm{n}-150}\left\{(\mathrm{vpy})_{2} \mathrm{vpyRe}(\mathrm{CO})_{3}\left(\mathrm{NO}_{2}-\text { phen }\right)^{+}\right\}_{\mathrm{m}-50}$ & 0.85 & $1.1 \times 10^{6}$ & 8.0 \\
\hline$\left\{(\mathrm{vpy})_{2} \mathrm{vpyRe}(\mathrm{CO})_{3}(\text { tmephen })^{+}\right)_{\mathrm{n} \sim 100}\left\{(\mathrm{vpy})_{2} \mathrm{vpyRe}(\mathrm{CO})_{3}\left(\mathrm{NO}_{2} \text {-phen }\right)^{+}\right\}_{\mathrm{m} \sim 100}$ & 0.94 & $3 \times 10^{6}$ & 6.7 \\
\hline
\end{tabular}

Tabla 8: Valores de Ej, $\mathrm{k}_{\mathrm{ET}}$ y $\bar{R}$ en los polímeros mixtos.

Estos valores de $\bar{R}$ pueden ser comparados con las distancias D-A de la figura 11 de distribución de distancias.

Para el polímero III, $\bar{R}=10.1 \AA$ es ligeramente menor que $\mathrm{R}_{\mathrm{F}}$ pero es $3 \AA$ mayor que la distancia mínima entre vecinos $7 \AA$. Para el polímero IV $\bar{R}=8.0 \AA$ implica que en promedio D y A están apartados sólo $1 \AA$ de la distancia mínima posible. En el polímero $\mathrm{V}$, sin embargo la distancia media entre cromóforos es similar a la mínima distancia entre vecinos y la eficiencia de la transferencia de energía es levemente mayor.

\section{ii. Luminiscencia resuelta en el tiempo}

En los experimentos de láser flash fotólisis de absorción, luego de la irradiación del polímero II, en solución de acetonitrilo con luz de $\lambda_{\text {exc }}=387 \mathrm{~nm}$, el transiente generado decae de manera monoexponencial muy rápidamente con un tiempo de vida de $\tau=230$ ps. El espectro de absorción del transiente consiste en tres bandas con máximos en 450 y $600 \mathrm{~nm}$ y $\lambda_{\max }>750 \mathrm{~nm}$.

De acuerdo a la similitud con los espectros de $\mathrm{TCML}_{\mathrm{Re} \rightarrow \mathrm{NO2} \text {-phen }}$ en los monómeros $\mathrm{LRe}(\mathrm{CO})_{3}\left(\mathrm{NO}_{2}\right)$-phen ${ }^{14}$ el transiente observado en la escala de tiempo de 
los picosegundos-nanosegundos en el polímero puede ser asignado al estado excitado de $\mathrm{TCML}_{\mathrm{Re} \rightarrow \mathrm{NO} 2 \text {-phen }}$ del cromóforo $\mathrm{Re}(\mathrm{CO})_{3}-\mathrm{NO}_{2}$-phen $)^{+}$decayendo no radiativamente.

En los polímeros I; III; IV yV (figura 7), puede apreciarse que $\Delta \mathrm{A}_{\text {máx }}$ decrece al pasar del I al V, aún cuando son corregidos por la disminución de cromóforos emisores $-\operatorname{Re}(\mathrm{CO})_{3} \mathrm{tmphen}^{+}$, que disminuye de 200 en I a 100 en $\mathrm{V}$.

Tomando los resultados experimentales se pueden calcular los valores:

$\Delta \mathrm{A}_{\operatorname{max~(I)}} / \Delta \mathrm{A}_{\operatorname{máx}(\mathrm{III})}: 1.7$

$\Delta \mathrm{A}_{\max (\mathrm{I})} / \Delta \mathrm{A}_{\max (\mathrm{IV})}: 3.4$

$\Delta \mathrm{A}_{\max (\mathrm{I})} / \Delta \mathrm{A}_{\operatorname{máx}(\mathrm{V})}: 5.2$

Mientras que sólo tomando la corrección por disminución en la concentración de cromóforos emisores los valores dan:

$\Delta \mathrm{A}_{\operatorname{máx}(\text { I) }} / \Delta \mathrm{A}_{\operatorname{máx}(\text { III) }} 1.1$

$\Delta \mathrm{A}_{\max (\mathrm{I})} / \Delta \mathrm{A}_{\max (\mathrm{IV})} 1.3$

$\Delta \mathrm{A}_{\max (\mathrm{l})} / \Delta \mathrm{A}_{\max (\mathrm{V})} 2.0$

$\Delta \mathrm{A}_{\text {máx }}$ puede ser considerado proporcional a la concentración de estados excitados de TCML fotogenerados en experimentos de láser flash fotólisis. Esta comparación sugiere que la desactivación de luminiscencia ocurre dentro de la vida del láser $(25 \mathrm{~ns})$ cuando los cromóforos $-\mathrm{Re}(\mathrm{CO})_{3}$ tmphen $^{+}$están situados dentro del radio crítico de Förster $\mathrm{R}_{\mathrm{F}}(10.7 \AA)$ de un aceptor $-\mathrm{Re}(\mathrm{CO})_{3}\left(5 \mathrm{NO}_{2} \text {-phen }\right)^{+}$y la manifestación de este quenching "instantáneo" es la disminución del $\Delta \mathrm{A}_{\text {máx }}$ mayor a la esperada por la disminución en la concentración de cromóforos luminiscentes.

Los cromóforos $\operatorname{Re}(\mathrm{CO})_{3}$ tmphen $^{+}$en estado excitado que se encuentran a una distancia mayor a $\mathrm{R}_{\mathrm{F}}$ del cromóforo aceptor serán desactivados con una constante $\mathrm{k}_{\mathrm{ET}}\left(\mathrm{D}_{\mathrm{i}}\right)$ y se observarán decayendo a tiempos mas largos que el tiempo de vida del pulso del láser. 
Los decaimientos de la absorción de los transientes de los polímeros I, III, IV y V son biexponenciales, y decaen en un período de tiempo de varios microsegundos.

Al comparar el decaimiento de la absorción del transiente del polímero I con el decaimiento de emisión del mismo luego de excitar con un láser de $\mathrm{N}_{2}$ de $\lambda_{\text {exc }}=337 \mathrm{~nm}$, puede notarse que el primero es biexponencial, mientras que el último es monoexponencial.

La diferencia reside en que mientras que lo experimentos de flash fotólisis de absorción fueron realizados con un láser excímero $\left(\lambda_{\mathrm{exc}}=351 \mathrm{~nm}\right)$, los experimentos de flash fluorescencia fueron realizados usando un láser de $\mathrm{N}_{2}\left(\lambda_{\text {exc }}=337 \mathrm{~nm}\right)$. La energía / pulso para el láser excímero es 15 veces mas alta que para experimentos de flash fluorescencia. Como consecuencia se produce una concentración de estado excitado de TCML mucho mas alta en experimentos de flash fotólisis de absorción que en experimentos de fluorescencia resuelta en el tiempo. Por lo tanto en experimentos de flash fotólisis de absorción puede producirse aniquilación entre estados de TCML que compita con el decaimiento de primer orden, debido a la presencia de cromóforos excitados muy próximos físicamente. Estas diferencias han sido informadas en literatura ${ }^{15}$.

El decaimiento de la absorción de los transientes en los polímeros mixtos fue biexponencial.

Como puede verse en la tabla 4 los dos tiempos de vida $\tau_{\text {rápido }}$ y $\tau_{\text {ñento }}$, decrecen al pasar del polímero III al V. Sin embargo el decaimiento del transiente probablemente, no sea verdaderamente biexponencial y los dos tiempos de vida correspondan a una distribución de tiempos de decaimiento los cuales son aproximadamnete ajustados como la suma de dos exponenciales y los dos tiempos de vida pueden representar la contribución promedio de cromóforos excitados en distinto ambiente. 


\section{Referencias}

1. Forster, T. Discuss. Faraday Soc. 1959, $27,7$.

2. Herz, L. M.; Silva, C.; Grimsdale, A. C.; Müllen, K; Phillips, R.T. Phys. Rev B 2004, 70, 165207.

3. Srinivas, G; Bagchi, B. J. Chem. Phys. 2002, 116, 837.

4. Rolinski, O. J.; Mathivanan, C.; Macnaught, G.; Birch, D. J. S. Biosensors and Bioelectronics 2004, 20, 424.

5. Tcherkasskaya, O.; Klushin, L.; Gronenborn, M. Biophysical Journal 2002, 82, 988.

6. Salthammer, T.; Dreeskamp, H.; Birch, D. J. S.; Imhof, R. E. J. Photochem. Photobiol. A: Chem. 1990, 55, 53

7. Birch, D. J. S.; Rolinski, O. J.; Hatrick, D. Rev. Sci. Instrum. 1996, 67, 2732.

8. Draxler, S. ; Lippitsch, M. E. Anal. Chem. 1996, 68, 753.

9. Birch, D. J. S.; Holmes, A. S.; Darbyshire, M. Meas. Sci. Technol. 1995, 6, 243.

10. Hallam, A. ; Birks, J. B. J. Phys. B: Atom. Molec. Phys. 1978, 11, 3273.

11. Turro, N.J. Modern Molecular Photochemistry, Mill Valley, Calif. : University Science Books, 1991, Chapter 9, p. 296.

12. Sonja Draxler; Max E. Lippitsch. Anal Chem. 1996, 68, 753-757.

13. E. N. Bodunov; M. Berberan-Santos; M. G. Martinho. Proceedings of $X X$ international conference on photochemistry. July 30-August 4, 2001, Moscow.

14. Busby, M.; Gabrielsson, A; Matousek, P. ;Towrie, M.;Di Bilio, A. J.;Gray,H. B.;vlcek,A. Jr. Inorg. Chem. 2004, 43,4994- 
Capítulo III

Reducción fotoinducida de Citocromo c por un polímero de $\mathbf{R e}^{\mathrm{I}}$ 


\section{Capitulo III}

\section{Introducción}

El Fe ${ }^{\text {III }}$-Cit c es una pequeña proteína de $120 \mathrm{kDa}$, que contiene 104 aminoácidos y un grupo hemo, esquema 1. Presenta una carga positiva de +9 a pH fisiológico. Los residuos de lisina cargados positivamente están homogéneamente distribuidos en la superficie de la proteína. La distribución de cargas negativas es asimétrica con los residuos cargados negativamente localizados en una pequeña área de la superficie del $\mathrm{Fe}^{\mathrm{III}}$-Cit c. ${ }^{1}$ Dicha distribución asimétrica de carga en Fe ${ }^{\mathrm{III}}-\mathrm{Cit} \mathrm{c}$ de corazón de caballo (Aldrich) resulta en un momento dipolar de $\sim 300 \mathrm{D}^{2}$ Este momento dipolar es creado por la ausencia de cargas negativas cercanas al solvente. ${ }^{3}$ El grupo hemo está unido covalentemente a Cys 14 y Cys 17 y el plano de la porfirina es perpendicular a la superficie de la proteína.

Esquema 1.
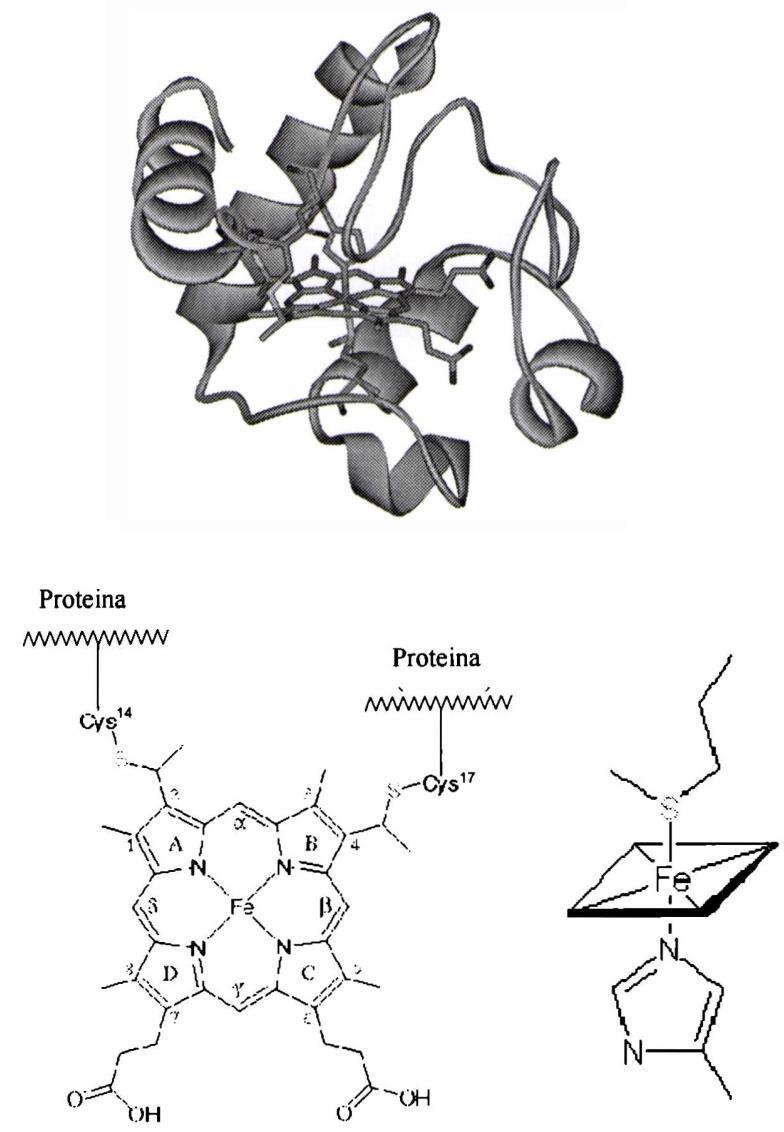
La proteína $\mathrm{Fe}^{\mathrm{III}}$-Cit $\mathrm{c}$ ha sido utilizada como proteina modelo para el estudio de evolución molecular. ${ }^{4 \mathrm{a}} \mathrm{La} \mathrm{Fe}^{\mathrm{III}}-\mathrm{Cit} \mathrm{c}$ es conocida como una proteína mitocondrial transportadora de electrones. La interconversión de $\mathrm{Fe}^{\mathrm{II}}$-Cit $\mathrm{c}$ y Fe $\mathrm{Fe}^{\mathrm{III}}$-Cit $\mathrm{c}$ dentro de la célula, juega un rol importa20nte en la oxidación celular tanto en plantas como en animales. A nivel celular la principal función es el transporte de electrones desde la citocromo c reductasa a la citocromo $\mathrm{c}$ oxidasa. La síntesis de $\mathrm{Fe}^{\mathrm{III}}$-Cit $\mathrm{c}$ ocurre en el espacio intermembrana de la mitocondria. Esto involucra la unión de la Apo Cit c, la cual es una estructura desordenada en solución con la posterior formación de la estructura plegada (nativa). La interacción de la estructura nativa $\mathrm{Fe}^{\mathrm{III}}-\mathrm{Cit} \mathrm{c}$ con lípidos resulta en una desnaturalización parcial de la proteína nativa, se ha demostrado que existe un equilibrio entre un estado soluble y un estado unido a membrana a $\mathrm{pH}$ fisiológico. $^{4 \mathbf{b}}$

Sin embargo, proteínas que son parcial o totalmente desordenadas bajo condiciones fisiológicas, pueden presentar funciones biológicas como reconocimiento, señalización y regulación. ${ }^{4 c}$

La transferencia de electrones intramolecular en $\mathrm{Fe}^{\mathrm{III}}$-Cit $\mathrm{c}$ ha sido investigada por la coordinación de complejos fotoactivos de $\mathrm{Ru}^{+2}$ a la superficie de la proteína. ${ }^{5-8}$ En estos estudios la transferencia de electrones fotoinducida ocurre entre el estado excitado reducido de $\operatorname{Ru}(\text { bpy })_{3}{ }^{+2}$ y el grupo hemo en su estado oxidado. Sin embargo no hay estudios de la transeferencia de electrones fotoinducida entre $\mathrm{Fe}^{\mathrm{III}}$-Cit $\mathrm{c}$ y complejos de $\operatorname{Re}^{\mathrm{I}}$. Complejos $\operatorname{Re}^{\mathrm{I}}(\mathrm{CO})_{3} \mathrm{LL}^{\prime}\left(\mathrm{L}^{\prime}=\right.$ diimina, $\mathrm{L}^{\prime}=$ haluro o piridina $)$ son capaces de actuar como fotocatalizadores con la ayuda de un reductor de sacrificio, ecuación 1. 


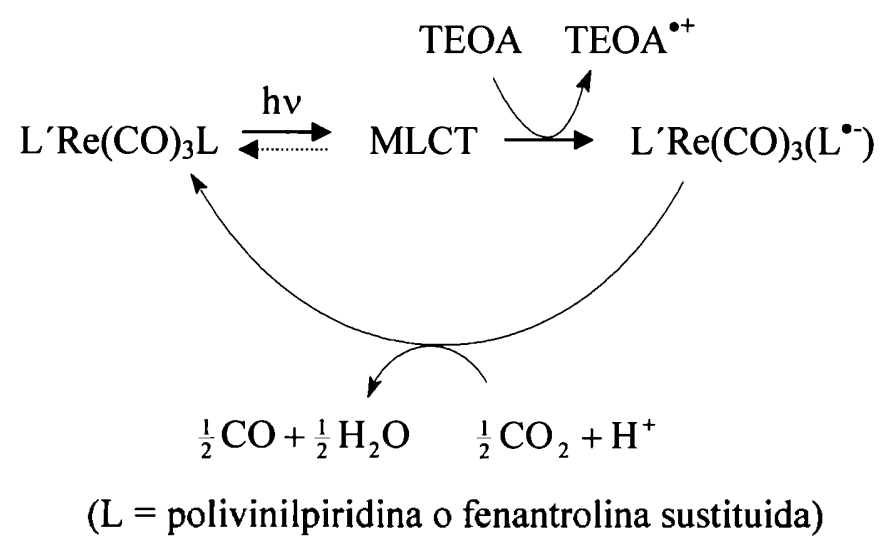

Es conocido que el quenching reductivo del estado de transferencia de carga metal ligando $\mathrm{TCML}_{\mathrm{Re} \rightarrow \mathrm{bpy}}$ por trietanolamina (TEOA) genera la especie $\left[\mathrm{ClRe}(\mathrm{CO})_{3}(\mathrm{bpy})\right]^{\bullet}$, capaz de mediar el proceso de reducción de 2 electrones de $\mathrm{CO}_{2}$ a $\mathrm{CO}^{9}$.

En este capítulo se desarrollarán los resultados obtenidos para la reducción fotoinducida de $\mathrm{Fe}^{\text {III }}-\mathrm{Cit} \mathrm{c}$ por el polímero $\left\{(\mathrm{vpy})_{2} \mathrm{vpyRe}(\mathrm{CO})_{3}(\text { tmephen })^{+}\right\}_{\mathrm{n} \sim 200}$ reducido en la mezcla de solventes $\mathrm{CH}_{3} \mathrm{CN} / \mathrm{H}_{2} \mathrm{O}$, donde la proteína está desnaturalizada. El quenching reductivo del polímero $\left\{(\mathrm{vpy})_{2} \mathrm{vpyRe}(\mathrm{CO})_{3}(\text { tmephen })^{+}\right\}_{n \sim 200}$ por trietilamina (TEA) produce la especie $-\operatorname{Re}(\mathrm{CO})_{3}(\mathrm{tmphen})^{\bullet}$ la cual en presencia de $\mathrm{Fe}^{\mathrm{III}}$-Cit $\mathrm{c}$, reduce el Fe del grupo hemo de su forma férrica a su forma ferrosa. 
1. Formación de aductos entre el polímero $\left\{(v p y)_{2} v p y \operatorname{Re}(\mathrm{CO})_{3}(\text { tmephen })^{+}\right\}_{n \sim 200}$ y el

\section{Citocromo c}

La formación de aductos entre $\left\{(\mathrm{vpy})_{2} \mathrm{vpyRe}(\mathrm{CO})_{3}(\text { tmephen })^{+}\right\}_{\mathrm{n} \sim 200}$ y el citocromo $\mathrm{c}$ ha quedado en evidencia a través de los cambios en el espectro UV-Visible, que se observan en soluciones de polímero mas citocromo $c$ en la mezcla de solventes acetonitrilo / agua (8:2 v:v) si se los compara con los espectros UV-Visibles de dichas especies tomados en forma individual.

Se realizó el espectro diferencia para soluciones conteniendo $8.8 \times 10^{-6} \mathrm{M}$ de citocromo c y varias concentraciones de $\left\{(\mathrm{vpy})_{2} \mathrm{vpyRe}(\mathrm{CO})_{3}(\mathrm{tmephen})^{+}\right\}_{\mathrm{n} \sim 200}$ variando la concentración de $\operatorname{Re}^{1},\left[\operatorname{Re}^{\mathrm{I}}\right]$ entre $8.8 \times 10^{-6} \mathrm{M}$ y $8.8 \times 10^{-5} \mathrm{M}$, figura 1. En la figura 2 se observa el espectro diferencia realizado con soluciones conteniendo $\left\{(\mathrm{vpy})_{2} \mathrm{vpyRe}(\mathrm{CO})_{3}(\text { tmephen })^{+}\right\}_{\mathrm{n} \sim 200}$ con $\left[\operatorname{Re}^{\mathrm{I}}\right]=8.8 \times 10^{-6} \mathrm{M}$ y varias concentraciones de citocromo c entre $2.2 \times 10^{-6} \mathrm{M}$ y $8.8 \times 10^{-6} \mathrm{M}$. El espectro diferencia fue calculado restando los espectros de $\left\{(\mathrm{vpy})_{2} \mathrm{vpyRe}(\mathrm{CO})_{3}(\text { tmephen })^{+}\right\}_{\mathrm{n}-200}$ y citocromo c tomados individualmente a los espectros de soluciones conteniendo ambas especies, en las mismas concentraciones que en las soluciones previas. 


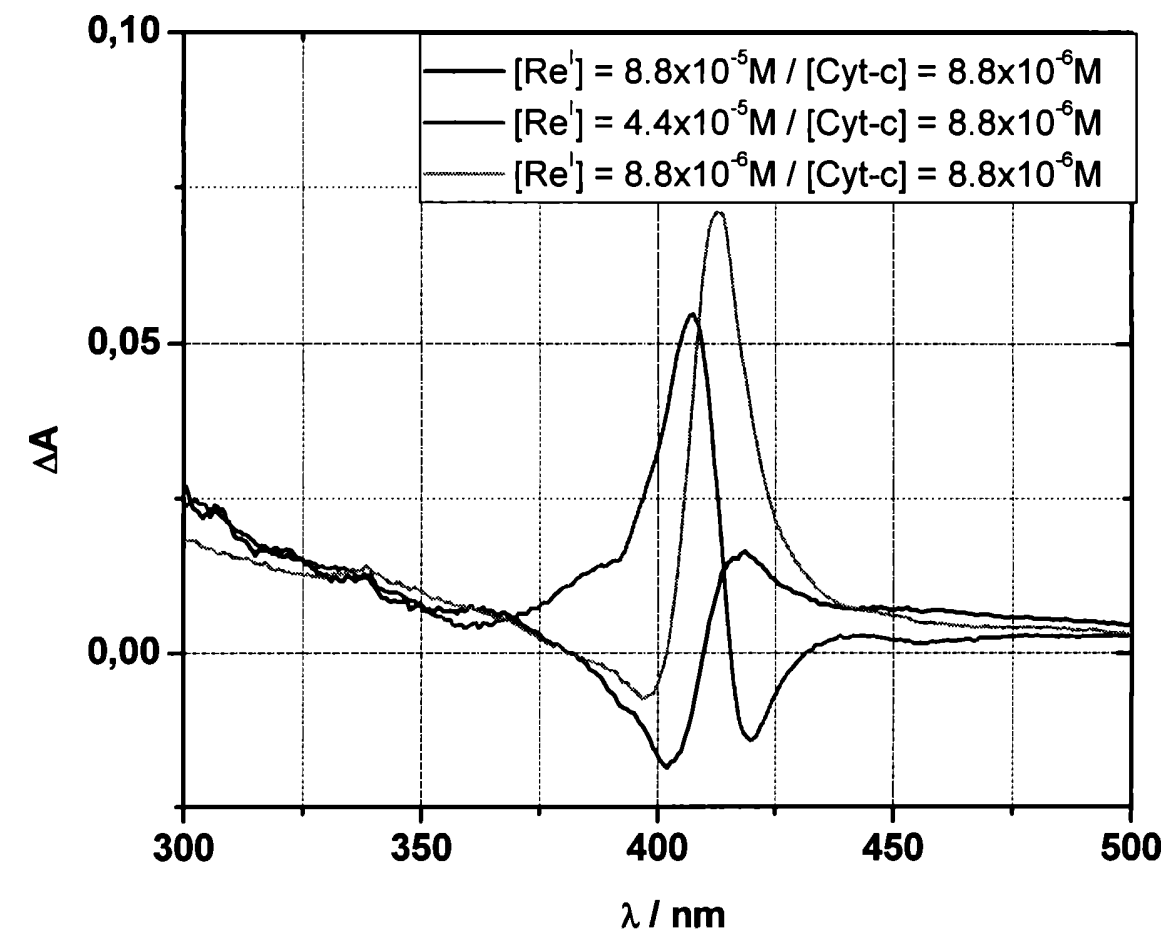

Figura 1: Diferencias espectrales registradas en soluciones con $8.8 \times 10^{-6} \mathrm{M} \mathrm{Fe}^{\mathrm{III}}$-Cit c y varias concentraciones de $\left\{\left[(\text { vpy })_{2} \operatorname{vpy} \operatorname{Re}^{\prime}(\mathrm{CO})_{3}(\text { tmphen })^{+}\right]\right\}_{\mathrm{n}-200}\left(\left[\mathrm{Re}^{\prime}\right]\right.$ entre $8.8 \times 10^{-6} \mathrm{M}$ y $\left.8.8 \times 10^{-5} \mathrm{M}\right)$. 


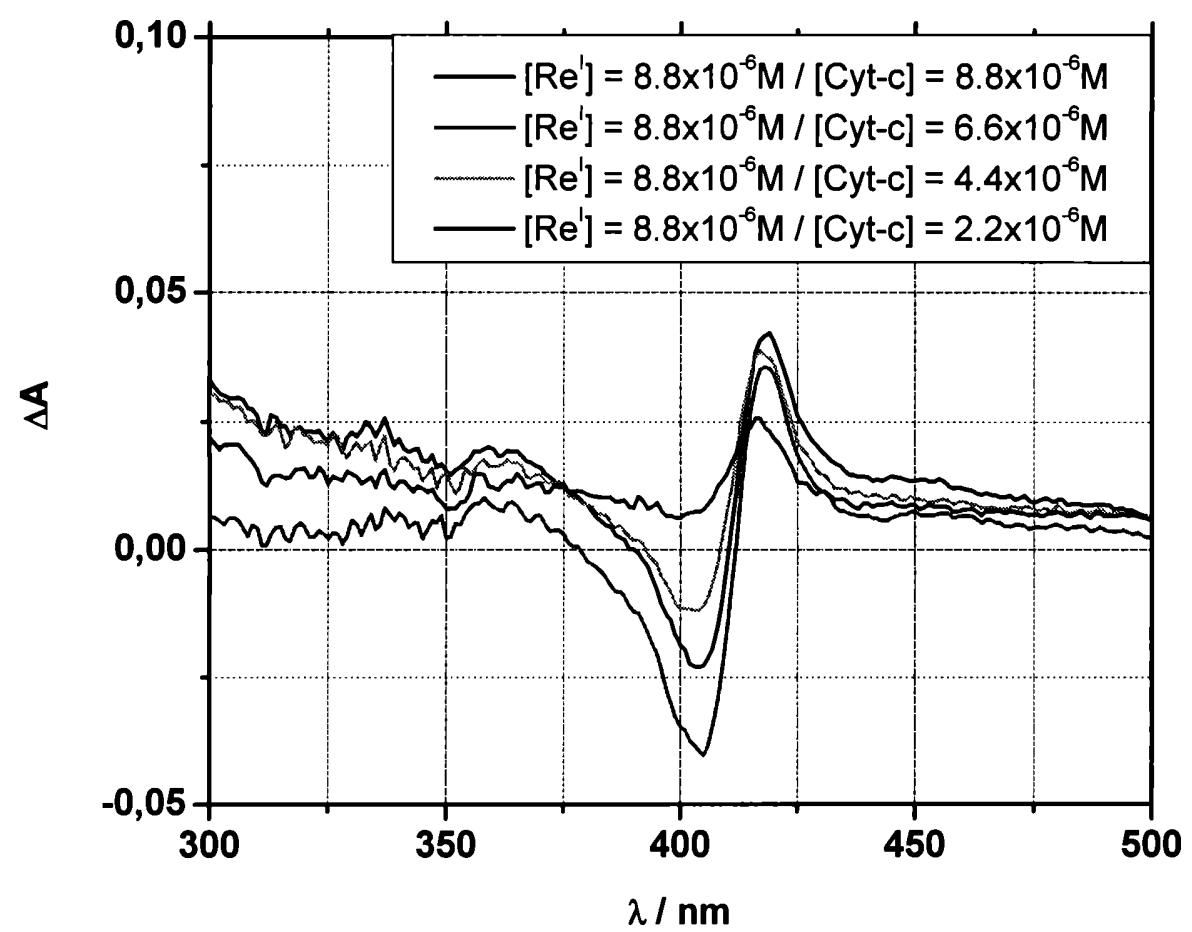

Figura 2: Diferencias espectrales registradas en soluciones con $\left\{\left[(\mathrm{vpy})_{2} \mathrm{vpyRe}^{\mathrm{I}}(\mathrm{CO})_{3}(\mathrm{tmphen})^{+}\right]\right\}_{\mathrm{n}-200}$ $\left(\left[\operatorname{Re}^{\mathrm{t}}\right]=8.8 \times 10^{-6} \mathrm{M}\right)$ y varias concentraciones de $\mathrm{Fe}{ }^{\mathrm{III}}$-Cit $\mathrm{c}$ entre $2.2 \times 10^{-6} \mathrm{M} \mathrm{y} 8.8 \times 10^{-6} \mathrm{M}$ 


\section{Morfologia de $\mathrm{Fe}^{I I I}-\mathrm{Cit} \mathrm{c},\left\{(\mathrm{vpy})_{2} v p y \operatorname{Re}(\mathrm{CO})_{3}(\text { tmephen })^{+}\right\}_{n-200}$ y del aducto}

La morfología del polímero $\left\{(\mathrm{vpy})_{2} \mathrm{vpyRe}(\mathrm{CO})_{3}(\text { tmephen })^{+}\right\}_{\mathrm{n} \sim 200}, \mathrm{Fe}^{\mathrm{III}}-\mathrm{Cit} \mathrm{c}$ y el aducto formado entre ellos, fue estudiada por microscopía electrónica de transmición (TEM). En las figuras 3 y 4 se muestran las morfologías obtenidas para films preparados a partir de la evaporación del solvente; acetonitrilo / agua (1:1 v:v). Los paneles a y b en la figura 3 muestran los films obtenidos a partir de soluciones del polímero. En la figura 4 panel a se muestra, el film obtenido a partir de soluciones de $\mathrm{Fe}^{\mathrm{III}}$-Cit $\mathrm{c}$, mientras que los paneles b y c muestran los films obtenidos a partir del aducto polímero-Cit c.

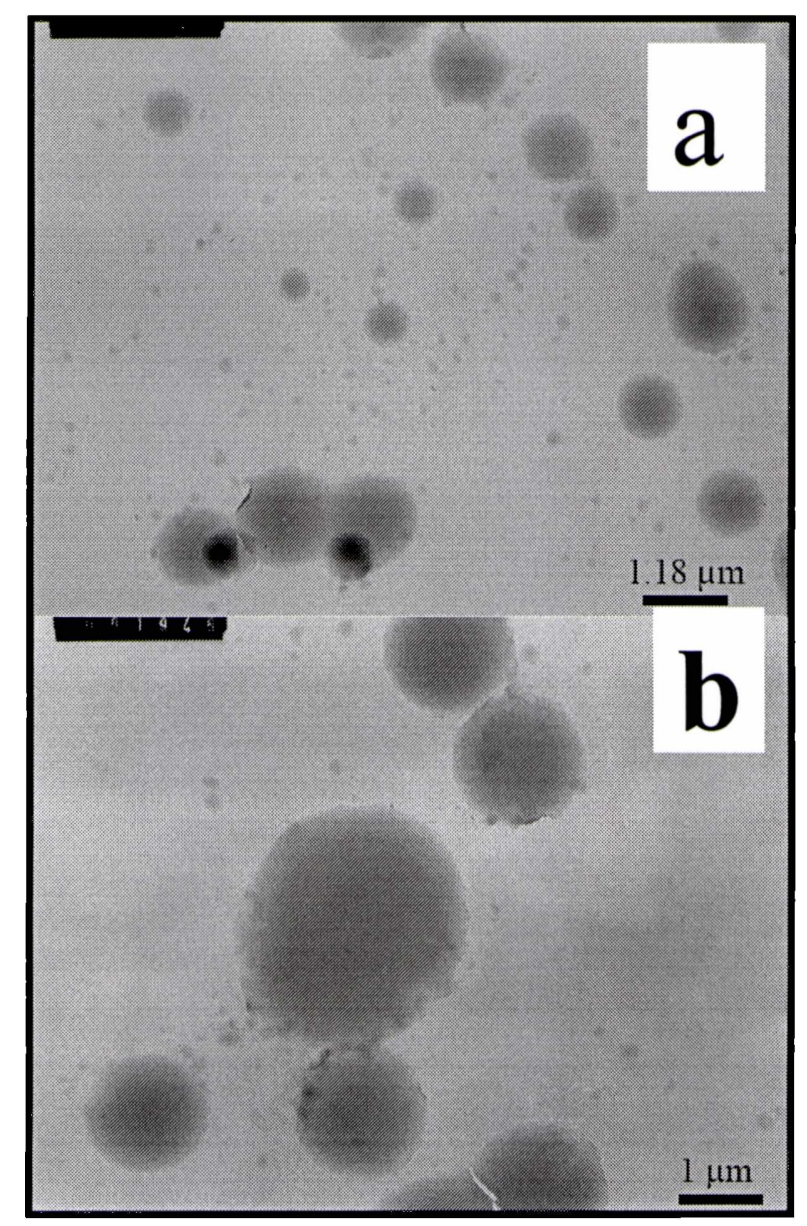

Figura 3: Films del polímero $\left\{\left[(\mathrm{vpy})_{2} \mathrm{vpyRe}^{\mathrm{l}}(\mathrm{CO})_{3}(\text { tmphen })^{+}\right]\right\}_{n-200}$ (panel a y b). Los films fueron preparados a partir de soluciones en $\mathrm{CH}_{3} \mathrm{CN} / \mathrm{H}_{2} \mathrm{O}(1: 1)$ con $\left[\mathrm{Re}^{\mathrm{I}}\right]=2.6 \times 10^{-4} \mathrm{M}$ 


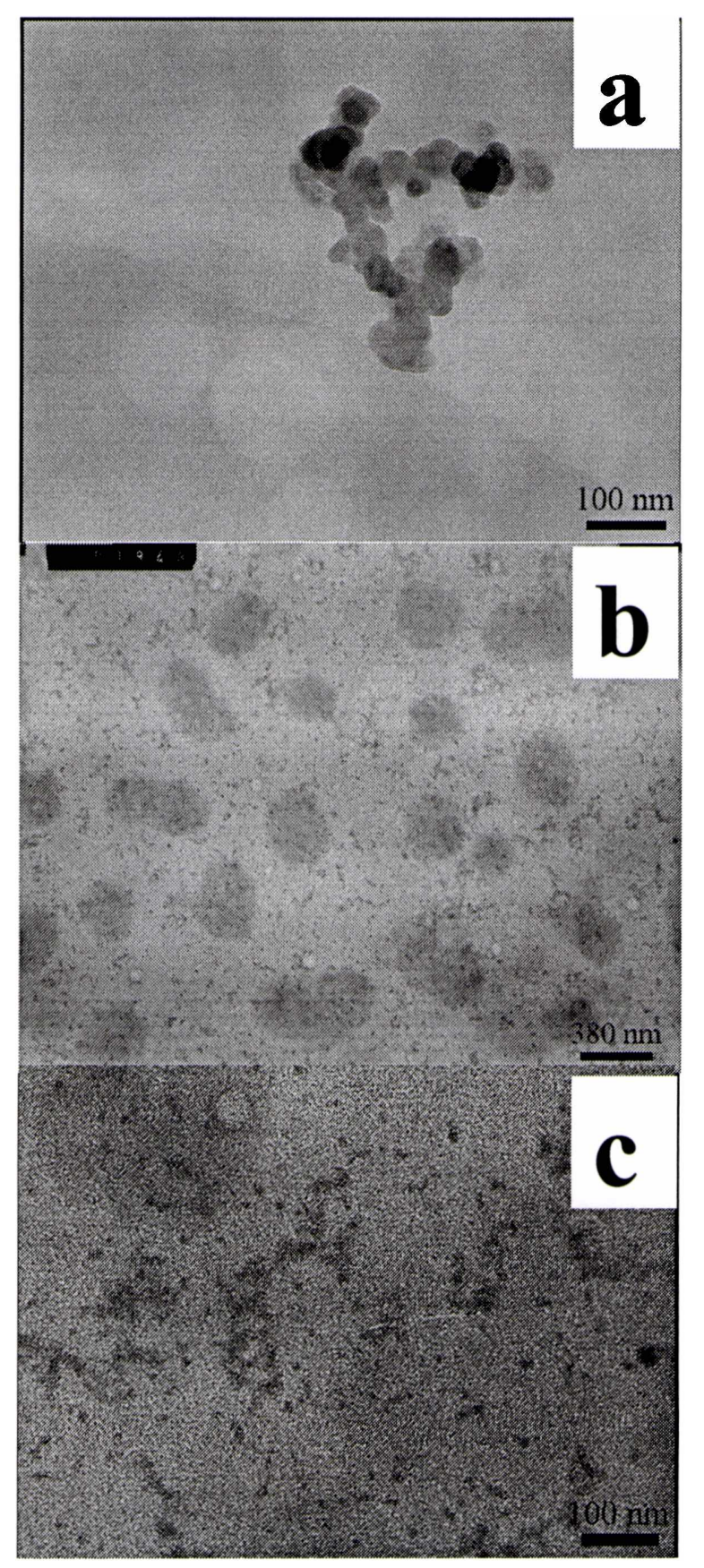

Figura 4: Films de $\mathrm{Fe}^{\mathrm{III}}$-Cit $\mathrm{c}$ (panel a) y del aducto entre $\left\{\left[(\mathrm{vpy})_{2} \mathrm{vpyRe}^{\prime}(\mathrm{CO})_{3}\left(\text { tmphen) }{ }^{+}\right]\right\}_{\mathrm{n}-200}\right.$ y Fe $\mathrm{Fe}^{\mathrm{III}}-\mathrm{Cit} \mathrm{c}$ (paneles b y c). Los films fueron preparados a partir de soluciones en $\mathrm{CH}_{3} \mathrm{CN} / \mathrm{H}_{2} \mathrm{O}(1: 1)$ con $\left[\mathrm{Re}^{\mathrm{l}}\right]=2.6 \times 10^{-4} \mathrm{M}$ y $\left[\mathrm{Fe}^{\mathrm{III}}-\mathrm{Cit} \mathrm{c}\right]=2.6 \times 10^{-4} \mathrm{M}$ 


\section{Especies reducidas de $\left\{(v p y)_{2} v p y \operatorname{Re}(\mathrm{CO})_{3}(\text { tmephen })^{+}\right\}_{n-200}$ vía fotoquímica y por} radiólisis de pulso

El espectro de absorción de los transientes del polímero $\left\{\left[(\mathrm{vpy})_{2} \mathrm{vpyRe}^{\mathrm{l}}(\mathrm{CO})_{3}(\mathrm{tmphen})^{+}\right]\right\}_{n \sim 200}$ fue registrado en un tiempo comprendido entre 15 ns y los microsegundos con un láser excímero XeF, de longitud de onda de excitación de $\lambda_{\text {exc }}: 351 \mathrm{~nm}$ y se mostró en la Parte II, Capítulo II de esta tesis. Esta longitud de onda de excitación produce la excitación óptica de la banda de absorción correspondiente a la TCML en el polimero de $\mathrm{Re}^{\mathrm{l}}$.

Para estudiar el quenching reductivo del estado excitado TCML se realizaron experimentos de láser flash fotólisis con soluciones de $\left\{(\mathrm{vpy})_{2} \mathrm{Vpy} \operatorname{Re}(\mathrm{CO})_{3}(\mathrm{tmephen})^{+}\right\}_{\mathrm{n} \sim 200}\left(\left[\mathrm{Re}^{\mathrm{I}}\right]=1.6 \times 10^{-4} \mathrm{M}\right)$ preparadas con acetonitrilo deaereado con $\mathrm{N}_{2}$ ultrapuro y en presencia de trietilamina TEA.

Se realizaron dos experimentos con concentraciones de TEA de 0.1 y $0.2 \mathrm{M}$. Los espectros registrados en experimentos de láser flash fotólisis se muestran en la figura 5 donde se puede observar que las bandas a $\lambda: 420 \mathrm{~nm}$ y $\lambda: 560 \mathrm{~nm}$ son de la misma intensidad cuando el quenching reductivo se realiza con TEA: $0.1 \mathrm{M}$. Sin embargo la banda a $420 \mathrm{~nm}$ es más intensa que la banda a $560 \mathrm{~nm}$ cuando la concentración de TEA utilizada es $0.2 \mathrm{M}$. El transiente generado en experimentos de láser flash fotólisis con las dos concentraciones de TEA decae con una cinética de primer orden. El tiempo de vida es $\tau: 6.3 \mu$ s y es independiente de la concentración de TEA.

Es posible atribuir el espectro de la figura 5 a la especie fotogenerada $-\operatorname{Re}^{\mathrm{I}}(\mathrm{CO})_{3}(\text { tmphen })^{\circ}$. Esta asignación puede ser confirmada debido a que el mismo cromóforo fue generado con la técnica de radiólisis de pulso que se realizó al polímero 
$\left\{(\mathrm{vpy})_{2} \mathrm{vpyRe}(\mathrm{CO})_{3}(\text { tmephen })^{+}\right\}_{n \sim 200}\left(\left[\operatorname{Re}^{\mathrm{I}}\right]=5.3 \times 10^{-5} \mathrm{M}\right)$ en $\mathrm{MeOH}$ deaereada con $\mathrm{N}_{2}$ ultrapuro. Como se describió en la Parte I, la radiólisis de soluciones preparadas en $\mathrm{CH}_{3} \mathrm{OH}$ y deaereadas con $\mathrm{N}_{2}$ ultrapuro, producen como principales productos $e_{\text {solv }}^{-}$y $\mathrm{CH}_{2} \mathrm{OH}^{\circ}$. Cuando las soluciones de polímero fueron deaereadas con $\mathrm{N}_{2} \mathrm{O}$ en lugar de $\mathrm{N}_{2}$, el pulso radiolítico no genera cambios en el espectro de la solución. Este experimento demuestra la falta de poder reductor del radical $\mathrm{CH}_{2} \mathrm{OH}^{*}$ sobre el ligando tmephen presente en el polímero de $\mathrm{Re}^{\mathrm{l}}$, y que los cambios que se reflejan en la figura 6 son generados por la reacción del polímero sólo con $e_{\text {solv }}^{-}$.

La reacción entre el $e_{s o l v}^{-}$, en experimentos de radiólisis de pulso y el polímero $\left\{(\mathrm{vpy})_{2} \mathrm{vpyRe}(\mathrm{CO})_{3}(\text { tmephen })^{+}\right\}_{n \sim 200}$ fue completa luego de los primeros microsegundos con una constante de velocidad de $(2.1 \pm 0.2) \times 10^{10} \mathrm{M}^{-1} \mathrm{~s}^{-1}$. El espectro generado por reacción del polímero con el $e_{\text {solv }}^{-}$se muestra en la figura 6 y este exhibe dos bandas de absorción con $\lambda_{\text {máx }}: 430 \mathrm{~nm}$ y $560 \mathrm{~nm}$, este espectro coincide con el espectro fotogenerado con TEA $=0.1 \mathrm{M}$. Una vez completa la reacción del $e_{\text {solv }}^{-}$con el polímero, las bandas de absorción a $\lambda_{\max }: 430$ y $560 \mathrm{~nm}$ presentan la misma intensidad. Sin embargo la banda a $\lambda_{\text {máx: }}: 560 \mathrm{~nm}$ decrece, relativa a la banda a $\lambda_{\text {máx }}: 430 \mathrm{~nm}$, luego de $3 \mathrm{~ms}$ del pulso. 


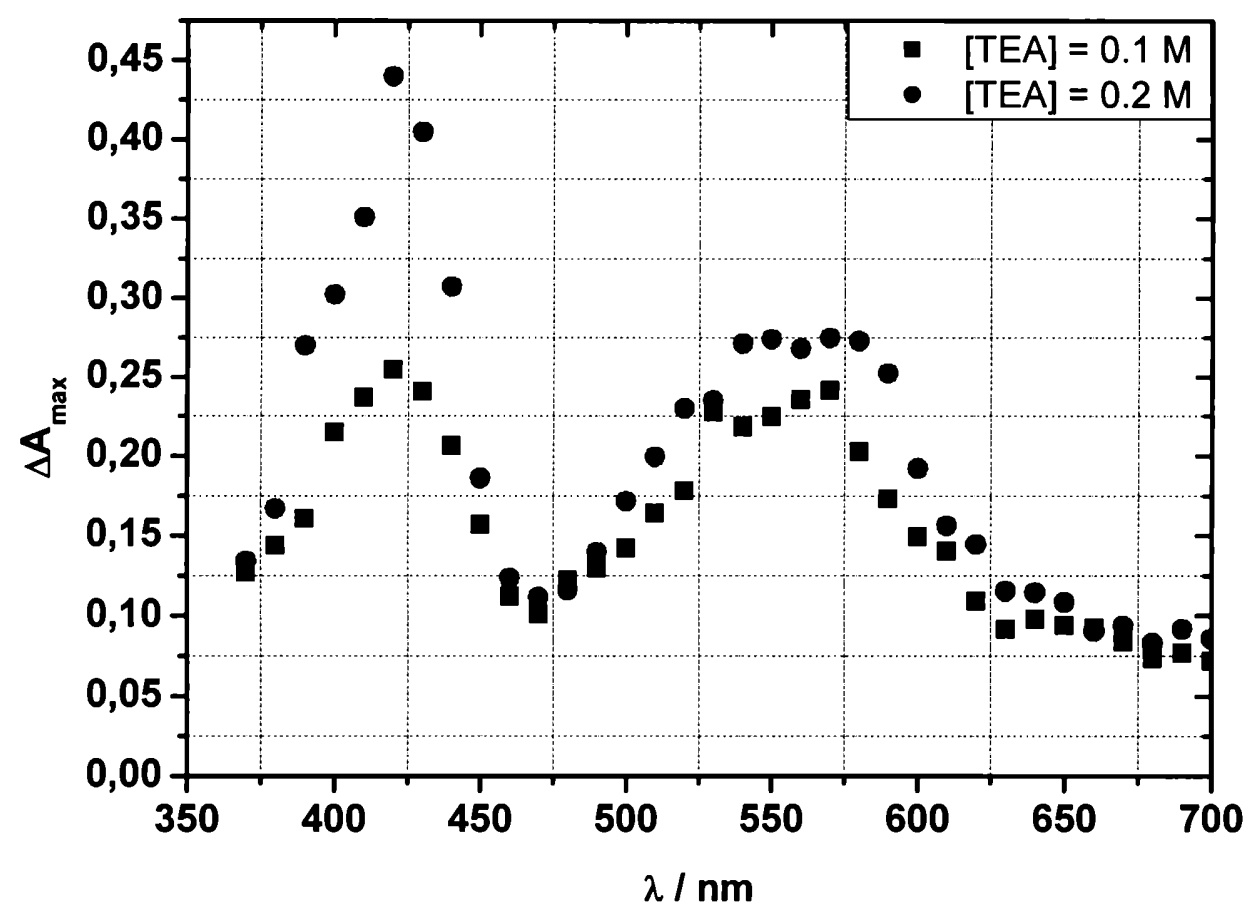

Figura 5: Espectro del transiente, registrado en experimentos de láser flash fotólisis sobre soluciones del polímero $\left\{\left[(\mathrm{vpy})_{2} \mathrm{vpyRe}(\mathrm{CO})_{3}(\text { tmphen })^{+}\right]\right\}_{n \sim 200}\left(\left[\mathrm{Re}^{\mathrm{l}}\right]=1.6 \times 10^{-4} \mathrm{M}\right)$ preparadas en $\mathrm{CH}_{3} \mathrm{CN}$ y deaereadas con $\mathrm{N} 2$ ultrapuro en presencia de TEA en concentraciones 0.1 y $0.2 \mathrm{M}$ 


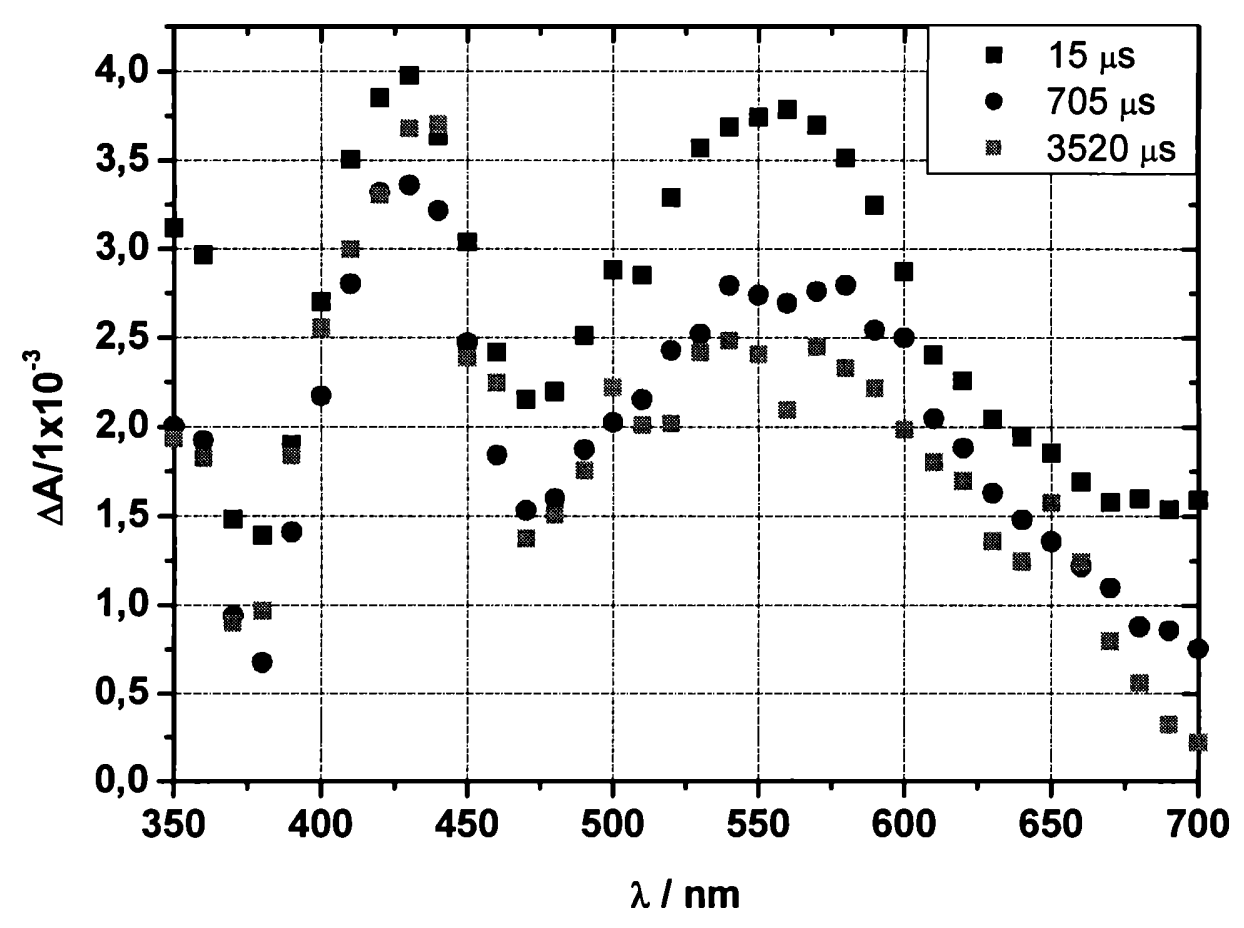

Figura 6: Espectro del transiente registrado luego de un pulso radiolítico en experimentos de radiólisis de pulso sobre soluciones metanólicas deaereadas con $\mathrm{N}_{2}$ de $\left\{\left[(\mathrm{vpy})_{2} \mathrm{vpyRe}^{\mathrm{I}}(\mathrm{CO})_{3}(\mathrm{tmphen})^{+}\right]\right\}_{\mathrm{n}-200}$ 


\section{Reducción fotoinducida del aducto de Fe ${ }^{I I \prime}$-Cit c con $\left\{(\text { vpy })_{2} v p y \operatorname{Re}(\mathrm{CO})_{3}(\text { tmephen })^{+}\right\}_{n \sim 200}$}

La reducción de $\mathrm{Fe}^{\mathrm{III}}$-Cit $\mathrm{c}$ a Fe ${ }^{\mathrm{II}}$-Cit $\mathrm{c}$ en el aducto fue investigada por medio de la técnica de láser flash fotólisis, con láser excímero de $\lambda_{\text {exc }}: 351 \mathrm{~nm}$ sobre soluciones deaereadas de $\left\{(\mathrm{vpy})_{2} \mathrm{vpyRe}(\mathrm{CO})_{3}(\text { tmephen })^{+}\right\}_{n \sim 200}\left(\left[\operatorname{Re}^{\mathrm{l}}\right]: 1.1 \times 10^{-4} \mathrm{M}\right)$, TEA $.06 \mathrm{M} \mathrm{y}$ $\mathrm{Fe}^{\mathrm{III}}$-Cit c : $\times 10^{-5} \mathrm{M}$ en una mezcla de solventes $\mathrm{CH}_{3} \mathrm{CN} / \mathrm{H}_{2} \mathrm{O}(50 \% \mathrm{v}: \mathrm{v})$. Los cambios fotoinducidos en el espectro de la solución muestran un incremento de $\mathrm{Fe}^{\mathrm{II}}-\mathrm{Cit} \mathrm{c}$ en función del tiempo luego del pulso del láser, figura 7. Las trazas oscilográficas que se muestran en la figura 8 revelan que una fracción de $\mathrm{Fe}^{\mathrm{II}}-\mathrm{Cit} \mathrm{c}$ se produce durante el pulso del láser. Para investigar si la irradiación de $\mathrm{Fe}^{\mathrm{III}}$-Cit $\mathrm{c}$ contribuye a los transientes observados en la figura 7 , se realizaron experimentos de flash fotoquímica similares con una solución "blanco" que contiene sólo Fe ${ }^{\mathrm{III}}$-Cit c y TEA. Luego de la irradiación de esta solución no se observó ningún cambio en la absorbancia.

La formación de $\mathrm{Fe}^{\mathrm{II}}$-Cit $\mathrm{c}$, figura 7, puede ocurrir por dos procesos, uno de ellos por quenching oxidativo del estado excitado de TCML del polímero por Fe $\mathrm{F}^{\mathrm{III}}$-Cit $\mathrm{c}$. Por otro lado el $\mathrm{Fe}^{\mathrm{II}}$-Cit $\mathrm{c}$ podría ser resultado de un proceso secundario, que ocurre luego del quenching reductivo del estado excitado de TCML del polímero por TEA. La flash irradiación de una solución "blanco" que contiene $\left\{(\mathrm{vpy})_{2} \mathrm{vpyRe}(\mathrm{CO})_{3}(\text { tmephen })^{+}\right\}_{\mathrm{n} \sim 200}\left([\mathrm{Re}]=1.1 \times 10^{-4} \mathrm{M}\right)$ y Fe $\mathrm{F}^{\mathrm{III}}-\mathrm{Cit} \mathrm{c}=5 \times 10^{-5} \mathrm{M}$ en $\mathrm{CH}_{3} \mathrm{CN}-\mathrm{H}_{2} \mathrm{O}(50 \% \mathrm{v}: \mathrm{v})$, genera el espectro del transiente del estado excitado de TCML de los pendientes de $\mathrm{Re}^{\mathrm{I}}$ presentes en el polímero. No se genera $\mathrm{Fe}^{\mathrm{II}}$-Cit $\mathrm{c}$ durante la irradiación,o como consecuencia del decaimiento del estado excitado. Por lo tanto se puede concluir que el $\mathrm{Fe}^{\mathrm{Il}}$-Cit $\mathrm{c}$ es formado por un proceso secundario que ocurre luego del quenching reductivo del estado excitado de TCML del polímero por TEA. 


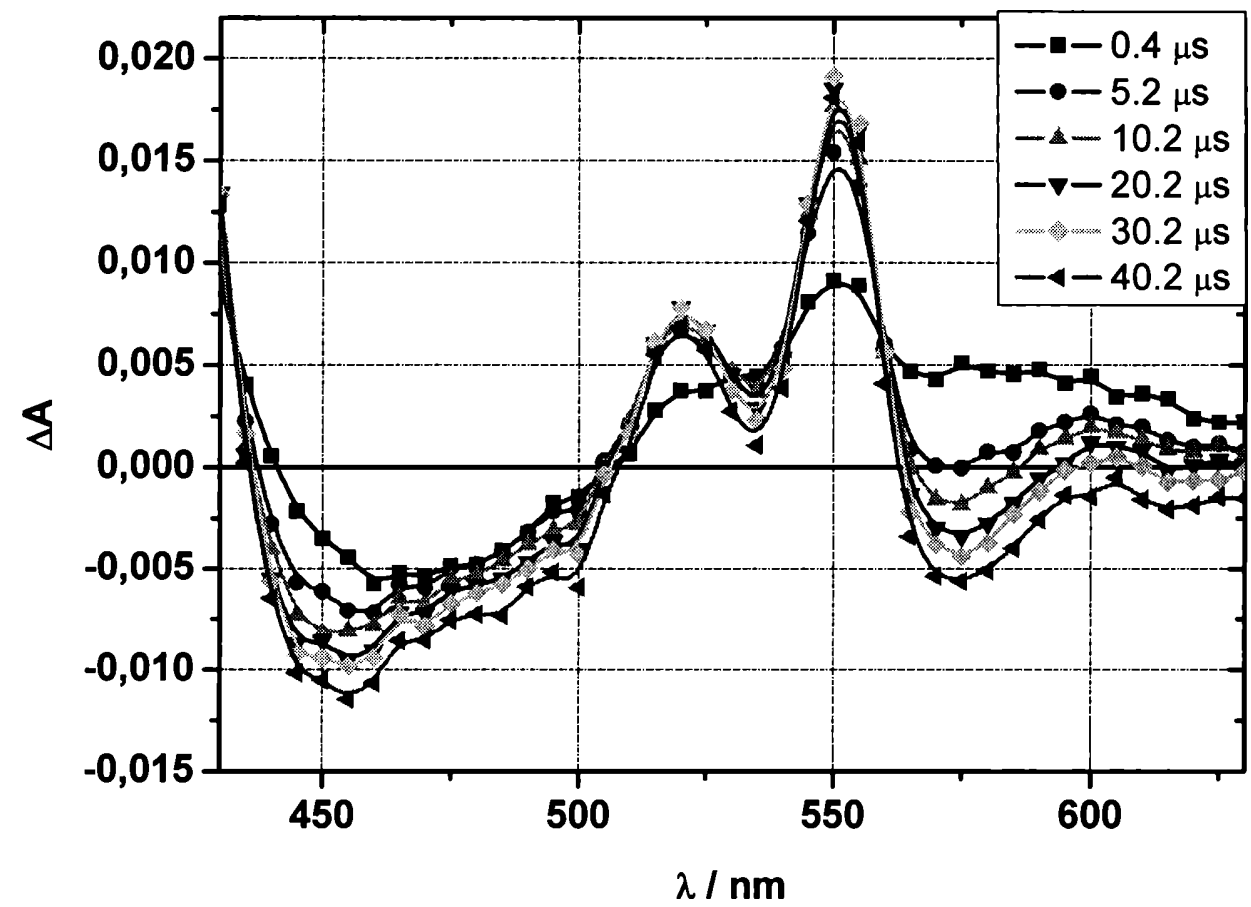

Figura 7: Espectro del transiente generado en experimentos de láser flash fotólisis $\left(\lambda_{\text {exc }}=351 \mathrm{~nm}\right)$ sobre soluciones deaereadas preparadas en $\mathrm{CH}_{3} \mathrm{CN} / \mathrm{H}_{2} \mathrm{O}\left(1: 1\right.$, v:v) y que contienen $\left\{\left[(\mathrm{vpy})_{2} \mathrm{vpyRe}(\mathrm{CO})_{3}(\text { tmphen })^{+}\right]\right\}_{\mathrm{n}-200}\left(\left[\operatorname{Re}^{\mathrm{I}}\right]=1.1\right.$ $\left.x 10^{-4} \mathrm{M}\right)$, TEA: $0.06 \mathrm{M} \mathrm{y} \mathrm{Fe}^{\mathrm{III}}-\mathrm{Cyt} \mathrm{c}: 5 \times 10^{-5} \mathrm{M}$ 


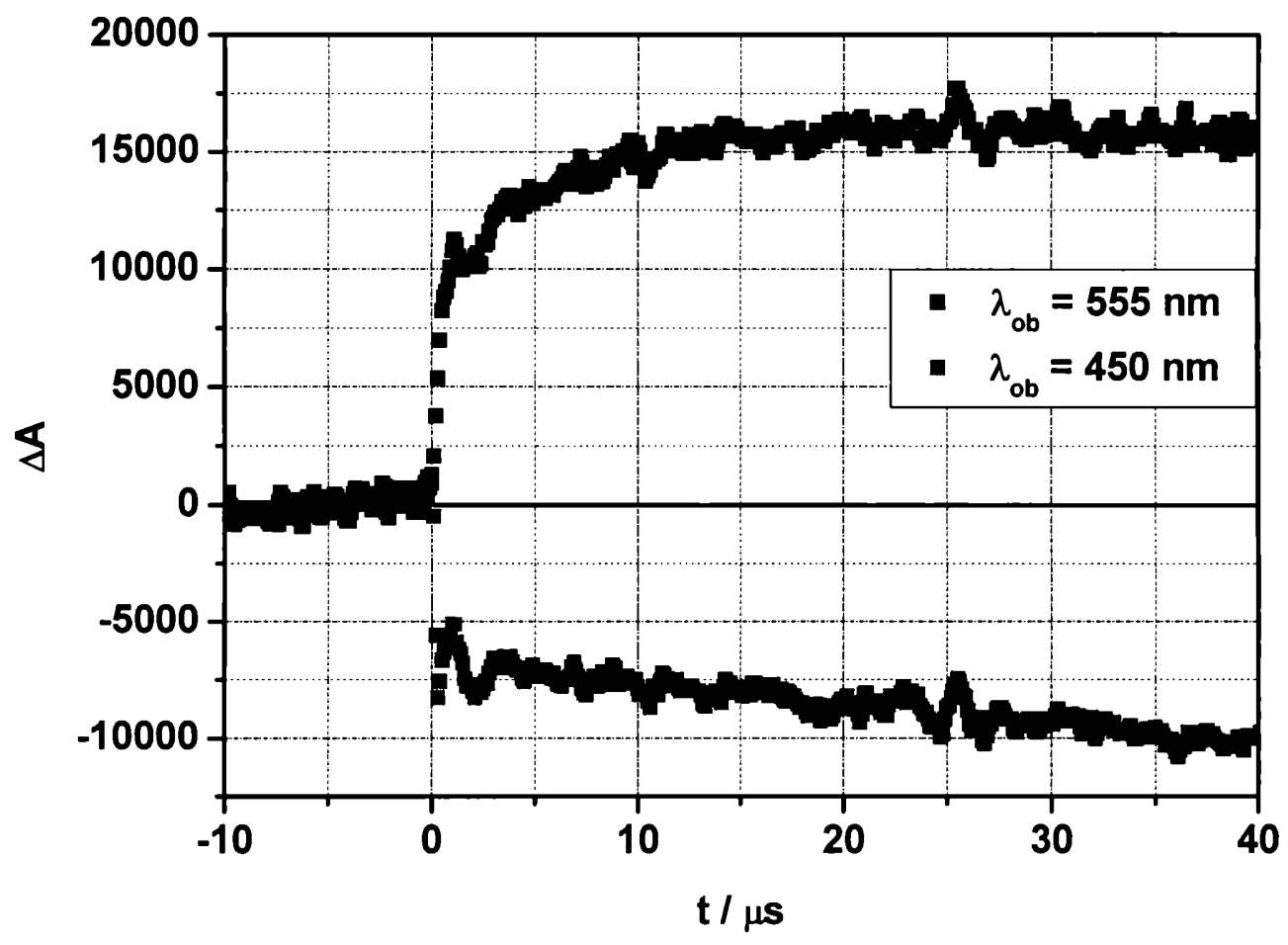

Figura 8: Traza oscilográfica registrada a $\lambda_{o b}=555$ and $450 \mathrm{~nm}$ en experimentos de láser flash fotólisis del sistema polímero de $\mathrm{Re}^{\mathrm{l}} / \mathrm{TEA} / \mathrm{Cit} \mathrm{c}$ en $\mathrm{CH}_{3} \mathrm{CN}-\mathrm{H}_{2} \mathrm{O}(1: 1)$ 


\section{Análisis espectral}

Para el análisis de los espectros resueltos en el tiempo se utilizó un software para técnicas quimométricas diseñado en nuestros laboratorios ${ }^{32 a}$ que trabaja según "multivariate self-modeling curve resolution" (MVCR) ${ }^{32 \mathrm{~b}}$. Estos métodos pueden aplicarse a datos de reacciones químicas con cinética espectroscópica bilineal, y provee información respecto a cambios en la composición y evolución del sistema. ${ }^{32 c}$ En este trabajo se ha elegido uno de los algoritmos mas ampliamente utilizados, la alternancia de cuadrados mínimos (ALS), que puede ayudar simultáneamente a estimar la concentración y el perfil espectral. ALS extrae información de la matriz de datos experimentales $\mathrm{A}(\mathrm{t} \times \mathrm{w})$ por aplicación iterativa del uso de análisis de regresión usando el siguiente producto de matrices:

$A=\mathbf{C S}^{\mathrm{T}}+\mathbf{E}$

Donde $C(t \times n)$ es la matriz del perfil cinético; $S^{T}(n \times w)$ contiene los perfiles espectrales, y $\mathrm{E}(\mathrm{t} \times \mathrm{w})$ representa la matriz error. Los números $\mathrm{t}, \mathrm{n}$, w denotan, tiempos de muestreo, número de especies absorbiendo y longitud de onda, respectivamente.

No es sencillo resolver la matriz $\mathrm{A}, \mathrm{n}$ es usualmente desconocido ${ }^{34 \mathrm{~b}}$ y por otro lado los métodos de resolución de curvas no otorgan una única solución debido a la ambigüedad rotacional y de escala. En este trabajo se ha aplicado el análisis de factores y descomposición de valores singular sobre la matriz experimental para la estimación de n, en virtud de reducir la ambiguiedad rotacional. 
El análisis de la diferencia espectral observada en la figura 7 fue realizado utilizando la técnica MVCR. Tanto el análisis de factores como la descomposición del valor angular fueron usados para la estimación del número de contribuciones independientes dando un valor de $n=2$. Los resultados se muestran en las figuras 9 y 10 La figura 9 muestra la diferencia espectral del primer factor en comparación con el espectro diferencia entre $\mathrm{Fe}^{\mathrm{III}}-\mathrm{Cit} \mathrm{c}$ y $\mathrm{Fe}^{\mathrm{II}}$-Cit $\mathrm{c}$ tomado de literatura. ${ }^{10-11} \mathrm{El}$ perfil espectral del segundo factor se muestra en la figura 10 en comparación con el espectro de $-\operatorname{Re}^{\mathrm{I}}(\mathrm{CO})_{3}(\text { tmphen })^{\bullet}$ obtenido por radiólisis de pulso y experimentos de láser flash fotólisis en presencia de TEA. Se puede notar que no hay un perfecto ajuste entre el perfil espectral del segundo factor y el espectro atribuible a la especie $-\operatorname{Re}^{\mathrm{l}}(\mathrm{CO})_{3}($ tmphen)', aunque la posición del valle y el máximo son coincidentes.

De acuerdo a la técnica de descomposición del valor angular la contribución espectral del segundo factor es mucho mas pequeña que la del primer factor, en correlación con la gran diferencia entre los valores del coeficiente de extinción molar de $-\operatorname{Re}^{\mathrm{I}}(\mathrm{CO})_{3}(\mathrm{tmphen})^{\circ}$ y $\mathrm{Fe}^{\mathrm{III}}$-Cit $\mathrm{c}$, ver mas adelante. Las diferencias observadas por debajo de $500 \mathrm{~nm}$ pueden ser debidas a una incompleta separación de las contribuciones espectrales, ambos factores están altamente acoplados en estas longitudes de onda. 


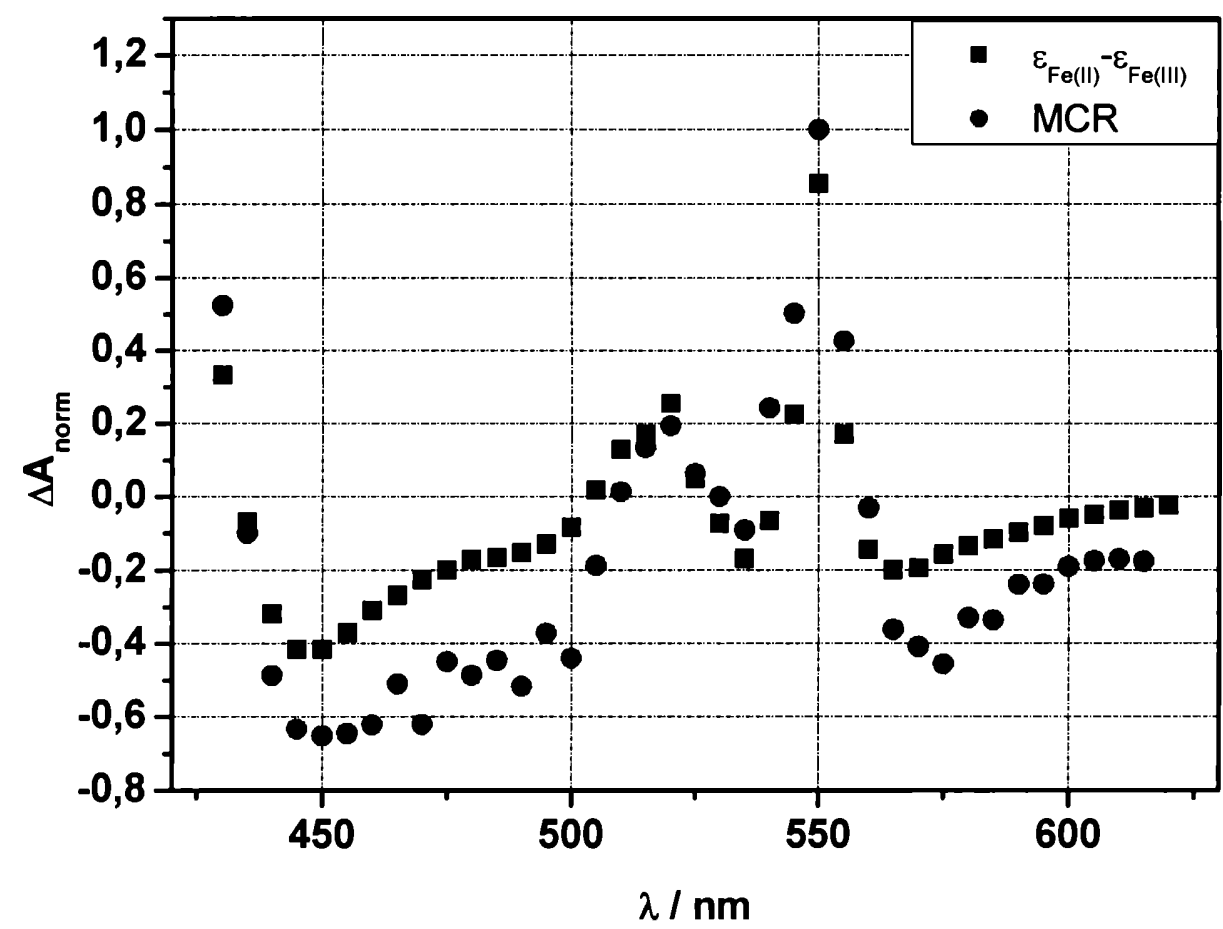

Figura 9: Espectro diferencia entre ferro y ferri-citocromo $\left(\varepsilon_{\mathrm{Fell}}-\varepsilon_{\mathrm{FellI}}\right)$ y espectro diferencia obtenido de la primera contribución, utilizando la técnica MVCR 


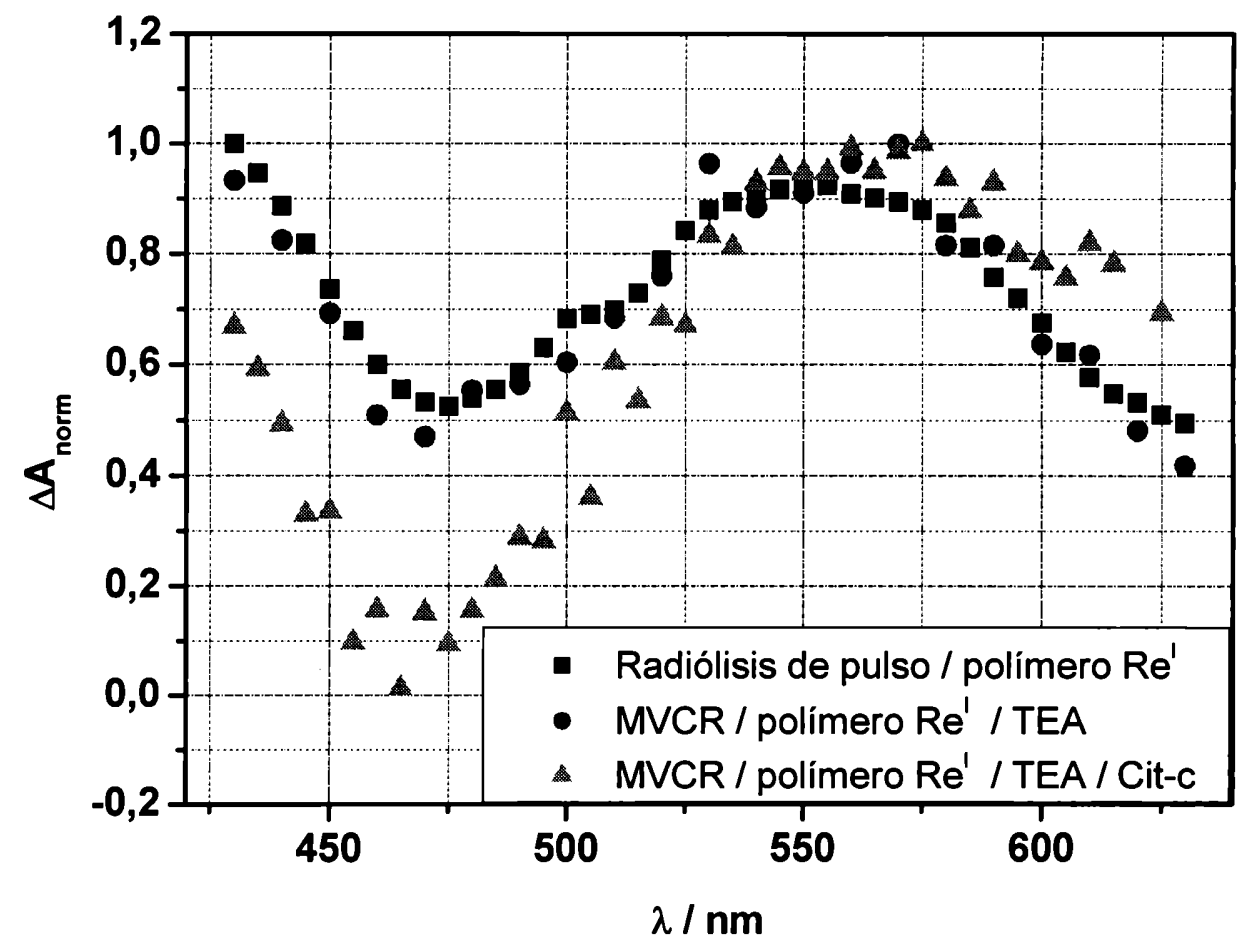

Figura 10: Comparación de los espectros diferencia registrados con radiólisis de pulso sobre soluciones en metanol deaereados con $\mathrm{N}_{2}$ y espectro diferencia de la segunda contribución usando la técnica MVCR 


\section{Discusión y conclusiones}

\section{i. Formación de aductos}

Los cambios observados en el espectro de absorción que se muestran en las figuras $1 \quad$ y $2 \quad 2$ revelaron la formación de aductos entre $\left\{(\mathrm{vpy})_{2} \mathrm{vpyRe}(\mathrm{CO})_{3}(\text { tmephen })^{+}\right\}_{\mathrm{n} \sim 200}$ y $\mathrm{Fe}^{\mathrm{III}}$-Cit c. Esta asociación probablemente involucra puentes de hidrógeno entre la lisina positivamente cargada del $\mathrm{Fe}^{\mathrm{III}}-\mathrm{Cit} \mathrm{c}$ y el átomo de nitrógeno libre de la piridina en $\left\{(\mathrm{vpy})_{2} \mathrm{vpyRe}(\mathrm{CO})_{3}(\text { tmephen })^{+}\right\}_{\mathrm{n}-200}$. Previamente ha sido postulada una estabilización de aductos similar, por puentes de hidrógeno, entre poli4-vinilpiridina y $\mathrm{Fe}^{\mathrm{III}}-\mathrm{Cit} \mathrm{c}{ }^{1}$

De recientes estudios cristalográficos ${ }^{12-13}$ se sabe que en el $\mathrm{Fe} \mathrm{e}^{\mathrm{III}}$-Cit $\mathrm{c}$ de corazón de caballo, el Fe se encuentra en el plano del anillo porfirínico con su quinta posición de coordinación ocupada por un átomo de nitrógeno del anillo imidazólico de la histidina 18, y este sitio permanece coordinado por un átomo de sulfuro de la metionina 80 .

Ligandos como azida, cianuro e imidazol coordinan al $\mathrm{Fe}$ y la evidencia sugiere que lo hacen por desplazamiento de la metionina $80 .{ }^{12-14-15} \mathrm{El}$ desplazamiento del átomo de sulfuro de la metionina 80 es evidenciado por una disminución en la absorbancia a $695 \mathrm{~nm}$ de la proteína. ${ }^{15}$

Los experimentos de unión entre el $\mathrm{Fe}^{\mathrm{III}}$-Cit $\mathrm{c}$ y $\left\{(\mathrm{vpy})_{2} \mathrm{vpyRe}(\mathrm{CO})_{3}(\mathrm{tmephen})^{+}\right\}_{\mathrm{n} \sim 200}$ que se muestran en las figuras 1 y 2 , se realizaron en la mezcla de solventes $20 \% \mathrm{H}_{3} \mathrm{CN} 80 \% \mathrm{H}_{2} \mathrm{O}$. Fue necesario agregar $\mathrm{CH}_{3} \mathrm{CN}$ para solubilizar el polímero. El $\mathrm{Fe}^{\mathrm{III}}$-Cit $\mathrm{c}$ se encuentra desnaturalizado en esta mezcla de solventes aún en ausencia de polímero. Los cambios espectrales mostrados en las figuras 1 y 2 no discriminan la coordinación de $\mathrm{Fe}^{\mathrm{III}}$ a piridinas libres de la unión por puentes de hidrógeno entre la proteína y el polímero. 


\section{ii. Microscopía electrónica de transmisión (TEM)}

La morfología de los compuestos estudiados,

$\left\{(\text { vpy })_{2} \mathrm{vpyRe}(\mathrm{CO})_{3}(\text { tmephen })^{+}\right\}_{\mathrm{n}-200}, \mathrm{Fe}^{\mathrm{III}}-\mathrm{Cit} \mathrm{c} \mathrm{y} \mathrm{el} \mathrm{aducto} \mathrm{se} \mathrm{muestran} \mathrm{en} \mathrm{la} \mathrm{figuras} 3 \mathrm{y}$ 4.

Todos los films fueron obtenidos a partir de soluciones preparadas en $\mathrm{CH}_{3} \mathrm{CN}$ / $\mathrm{H}_{2} \mathrm{O}$. La figura 3 , paneles a y b muestran que en la fase sólida de $\left\{(\mathrm{vpy})_{2} \mathrm{vpyRe}(\mathrm{CO})_{3}(\text { tmephen })^{+}\right\}_{n \sim 200}$ los complejos de $\operatorname{Re}^{\mathrm{I}}$ se agregan y forman nanodominios. Los tamaños son muy polidispersos con un rango de diámetros entre 140 nm y $2.8 \mu \mathrm{m}$. El largo de cadena de $\left\{(\mathrm{vpy})_{2} \mathrm{vpyRe}(\mathrm{CO})_{3}(\mathrm{tmephen})^{+}\right\}_{\mathrm{n} \sim 200}$ es en promedio $\sim 200 \mathrm{~nm}$, por lo que se puede inferir que los nanodominios deben corresponder a agregados de muchas cadenas.

La figura 4, panel a muestra agregados esféricos de $\mathrm{Fe}^{\mathrm{III}}-\mathrm{Cit} \mathrm{c}$, con diámetro alrededor de 30-50 nm, las dimensiones de estos nanodominios son considerablemente mas grandes que el diámetro del $\mathrm{Fe}^{\mathrm{III}}$-Cit $\mathrm{c}$, que es alrededor de $4 \mathrm{~nm}$, por lo tanto los nanodominios están compuestos por varias moléculas de proteína.

La figura 4, paneles b y c muestran la morfología de los aductos, y esta es diferente a la observada para el polímero solo. Para el caso del aducto, los grandes nanodominios desaparecen y aparecen nanodominios de una simetría esférica distorsionada de alrededor de $300 \mathrm{~nm}$ de diámetro. Estos cambios de tamaño, sugieren que el número de cadenas formando los nanodominios de $\left\{(\mathrm{vpy})_{2} \mathrm{vpyRe}(\mathrm{CO})_{3}(\text { tmephen })^{+}\right\}_{\mathrm{n} \sim 200}$ disminuye debido a la asociación del polímero con $\mathrm{Fe}^{\mathrm{III}}$-Cit $\mathrm{c}$. En la figura 4, panel c, puede notarse que hay además agregados de $\mathrm{Fe}^{\mathrm{III}}-\mathrm{Cit}$ $\mathrm{c}$ en adición a los agregados de polímero $\left\{(\mathrm{vpy})_{2} \mathrm{vpyRe}(\mathrm{CO})_{3}(\mathrm{tmephen})^{+}\right\}_{n \sim 200}$.

La formación y agregación de aductos puede representarse por las siguientes ecuaciones: 


$$
\begin{aligned}
& m\left\{\left[(\mathrm{vpy})_{2} \mathrm{vpyRe}^{\mathrm{I}}(\mathrm{CO})_{3}(\mathrm{tmphen})^{+}\right]\right\}_{\mathrm{n} \sim 200} \stackrel{\mathrm{CH}_{3}\left(\mathrm{CN} / \mathrm{H}_{2} \mathrm{O}\right.}{\longrightarrow}\left\langle\left\{\left[(\mathrm{vpy})_{2} \mathrm{vpyRe}^{\mathrm{I}}(\mathrm{CO})_{3}(\mathrm{tmphen})^{+}\right]\right\}_{\mathrm{n} \sim 200}\right\rangle_{m} \\
& N \mathrm{Fe}^{I I I}-\mathrm{Cyt} \mathrm{c} \stackrel{\mathrm{CH} \mathrm{CN}_{3} \mathrm{H}_{2} \mathrm{O}}{\longrightarrow}\left(\mathrm{Fe}^{I I I}-\mathrm{Cyt} c\right)_{N} \\
& \text { Proteína nativa } \\
& \text { desnaturalización y } \\
& \text { agregación }
\end{aligned}
$$

$$
\left\langle\left\{\left[(\mathrm{vpy})_{2} \operatorname{vpyRe}^{\prime}(\mathrm{CO})_{3}(\mathrm{tmphen})^{+}\right]\right\}_{\mathrm{n} \sim 200}\right\rangle_{m}+\left(\mathrm{Fe}^{I I I}-\mathrm{Cyt} \mathrm{c}\right)_{N} \rightarrow \text { Aductos }
$$

En conclusión, la polidispersidad de los nanoagregados de $\left\{(\mathrm{vpy})_{2} \text { vpyRe}(\mathrm{CO})_{3}(\text { tmephen })^{+}\right\}_{\mathrm{n} \sim 200}$ es muy alta con diámetros que varían entre $140 \mathrm{~nm}$ a $3 \mu \mathrm{m}$. La formación de aductos entre el polímero y el $\mathrm{Fe}^{\mathrm{III}}$-Cit c reduce el tamaño de los nanoagregados de $\left\{(\mathrm{vpy})_{2} \mathrm{vpyRe}(\mathrm{CO})_{3}(\text { tmephen })^{+}\right\}_{\mathrm{n} \sim 200}$ a esferas distorsionadas de $300 \mathrm{~nm}$. Los aductos pueden contener unidades discretas de $\mathrm{Fe}^{\mathrm{III}}-\mathrm{Cit} \mathrm{c}$ unidas o en forma de agregados $\left(\mathrm{Fe}^{\mathrm{III}}-\mathrm{Cit} \mathrm{c}\right)_{N}$ interaccionando con las cadenas del polímero $\left\{(\text { vpy })_{2} \operatorname{vpyRe}(\mathrm{CO})_{3}(\text { tmephen })^{+}\right\}_{n \sim 200}$

\section{iii. Reacciones redox del estado fundamental}

El espectro del transiente luego de la reacción entre $e_{\text {solv }}^{-} \quad \mathrm{y}$ $\left\{(\mathrm{vpy})_{2} \mathrm{vpyRe}(\mathrm{CO})_{3}(\text { tmephen })^{+}\right\}_{\mathrm{n}-200}(15 \mu$ s en la figura 6$)$ puede ser asociada con la especie $\operatorname{Re}(\mathrm{CO})_{3}(\text { tmephen })^{*}$ en el polímero $\left\{(\mathrm{vpy})_{2} \operatorname{vpyRe}(\mathrm{CO})_{3}(\text { tmephen })^{+}\right\}_{\mathrm{n} \sim 200}$. La concentración de $e_{s o l v}^{-}$generada en experimentos de radiólisis de pulso $\left[e_{s o l v}^{-}\right] \sim 2 \times 10^{-6}$ 
$\mathrm{M}$, por lo tanto sólo un pequeño porcentaje $(\sim 4 \%)$ del número total de cromóforos $\operatorname{Re}(\mathrm{CO})_{3}(\text { tmephen })^{+}$son reducidos a $-\operatorname{Re}(\mathrm{CO})_{3}(\text { tmephen })^{\bullet}$ por el $e_{\text {solv }}^{-}$en el polímero de $\operatorname{Re}^{\mathrm{I}}$. Los cambios en el espectro de absorción dentro de los milisegundos, $3.5 \mathrm{~ms}$, figura 6, pueden ser interpretados como la desaparición del radical - $\operatorname{Re}(\mathrm{CO})_{3}(\text { tmephen })^{\circ}$. Dicha desaparición de radicales puede ser entendida como una lenta reacción de desproporcionación, que requiere el desplazamiento difusivo de las cadenas de polímero

\section{iv. Procesos redox del estado excitado del polímero en ausencia de Fe ${ }^{I I I}$-Cit c}

La reducción del estado excitado del polímero por TEA se lleva a cabo en un tiempo menor a 25 ns. No se ha observado un crecimiento del espectro de $\operatorname{Re}(\mathrm{CO})_{3}(\text { tmephen })^{\bullet}$, derivado de la reducción de grupos $\operatorname{Re}(\mathrm{CO})_{3}(\text { tmephen })^{+}$por la oxidación con radicales reductores derivados de la oxidación de la TEA.

El crecimiento del espectro de $\operatorname{Re}(\mathrm{CO})_{3}(\text { tmephen })^{\bullet}$ es seguido por un rápido decaimiento con una cinética de primer orden y un $\tau=6.2 \mu \mathrm{s}$. El espectro de la figura 6 , registrado en experimentos de radiólisis de pulso, es similar al de la figura 5 obtenido con $[\mathrm{TEA}]=0.1 \mathrm{M}$, asociado con el producto inicial de reducción, $-\operatorname{Re}(\mathrm{CO})_{3}(\mathrm{tmephen})^{*}$. De la comparción de $\Delta \mathrm{A}_{\text {max }}$ de las figuras 5 y 6 se puede estimar que la concentración de $\operatorname{Re}(\mathrm{CO})_{3}(\text { tmephen })^{\circ}$ generada en experimentos de flash fotólisis con TEA es dos órdenes de magnitud mayor que la concentración de radicales generada por experimentos de radiólisis de pulso; $\left[\operatorname{Re}(\mathrm{CO})_{3}(\text { tmephen })^{\circ}\right]_{\text {nlash fotólisis }} \sim 1 \times 10^{-4} \mathrm{M}$.

La forma del espectro obtenido en la figura 5 con $[$ TEA] $=0.2 \mathrm{M}$, es similar al espectro obtenido en experimentos de radiólisis de pulso a tiempos mas largos. Sin embargo ambos transientes decaen con la misma cinética. Es posible que ocurra una reacción secuencial de desproporcionación de radicales $\operatorname{Re}(\mathrm{CO})_{3}($ tmephen); ecuaciones 4 y 5 pueden ocurrir secuencialmente ${ }^{10}$ 
$-\operatorname{Re}^{\mathrm{l}}(\mathrm{CO})_{3}(\mathrm{tmphen})^{\bullet}+\mathrm{TEAH}^{+} \rightarrow \operatorname{Re}^{\mathrm{I}}(\mathrm{CO})_{3}(\mathrm{tmphenH})^{\bullet+}+\mathrm{TEA}$

$2-\operatorname{Re}^{\mathrm{I}}(\mathrm{CO})_{3}(\operatorname{tmphenH})^{\bullet+} \rightarrow \operatorname{Re}^{\mathrm{I}}(\mathrm{CO})_{3}\left(\mathrm{tmphenH}_{2}\right)^{+}+\operatorname{Re}^{\mathrm{I}}(\mathrm{CO})_{3}(\mathrm{tmphen})^{+}$

Estas ecuaciones, 4 y 5 , representan la protonación de $-\operatorname{Re}(\mathrm{CO})_{3}(\text { tmephen })^{\circ}$ por $\mathrm{TEAH}^{+}$en el polímero, un lento movimiento difusional de las cadenas poliméricas permite el encuentro bimolecular de pendientes $-\operatorname{Re}(\mathrm{CO})_{3}(\operatorname{tmephenH})^{\circ+}$. La especie $\mathrm{TEAH}^{+}$es producida por desproporcionación del radical catión TEA ${ }^{\bullet+},\left(\mathrm{CH}_{3} \mathrm{CH}_{2}\right)_{3} \mathrm{~N}^{\circ+}$, ecuación 6:

$\left(\mathrm{CH}_{3} \mathrm{CH}_{2}\right)_{3} \mathrm{~N}^{\bullet+}+\mathrm{TEA} \rightarrow\left(\mathrm{CH}_{3} \mathrm{CH}_{2}\right)_{2} \mathrm{~N}\left(\mathrm{CH}_{2} \mathrm{CH}_{2}{ }^{\circ}\right)+\mathrm{TEAH}^{+}$

El proceso total ocurre en la escala de milisegundos-segundos en experimentos de radiólisis de pulso donde la especie $\operatorname{Re}(\mathrm{CO})_{3}(\text { tmephen })^{\bullet}$ es producida en baja concentración $\left(\sim 2 \times 10^{-6}\right)$. Por esta razón, los radicales se encuentran separados y son necesarios movimientos difusivos de las cadenas de polímero para que se produzca el encuentro. Sin embargo, en experimentos de flash fotólisis, la concentración inicial de especies reducidas, $\operatorname{Re}(\mathrm{CO})_{3}(\text { tmephen })^{\circ}$ generadas por quenching reductivo del estado excitado por TEA, es muy alta, entre 80 y $90 \%$ del total de $\operatorname{Re}(\mathrm{CO})_{3}(\text { tmephen })^{+}$. En esta condición la desproporcionación ocurre mucho más rápido, en la escala de los milisegundos. Además, la similitud entre los espectros generados por flash fotólisis y radiólisis de pulso sugieren que a bajas concentraciones de TEA, predomina el decaimiento de la especie $\operatorname{Re}(\mathrm{CO})_{3}(\text { tmephen })^{\circ}$. Mientras que a concentración de TEA 
mas alta, la especie transiente predominante es la especie protonada $\operatorname{Re}(\mathrm{CO})_{3}(\text { tmephen })^{-+}$.

\section{v. Reacciones redox del aducto polímero-Fe ${ }^{I I I}$-Cit c}

Como puede observarse en la figura 7 la reducción fotoinducida de $\mathrm{Fe}^{\mathrm{II}}-\mathrm{Cit} \mathrm{c}$ a $\mathrm{Fe}^{\mathrm{II}}$-Cit c ocurre en una escala de tiempo de $40 \mu$ s por un proceso de dos pasos.

Inicialmente se incrementa la absorbancia a $\lambda=555 \mathrm{~nm}$, característica del ferrocitocromo, al mismo tiempo que disminuye la absorbancia a $\lambda=450 \mathrm{~nm}$, característica del ferricitocromo. Una primera reducción ocurre dentro de la vida del láser Una reducción mas lenta ocurre en una escala de tiempo entre 1-40 $\mu \mathrm{s}$. El análisis del perfil de concentración obtenido para el primer factor con la técnica MVCR, muestra que el proceso mas lento es cinéticamente de primer orden con un tiempo de vida de $\tau=(15 \pm 1) \mu \mathrm{s}$. El proceso de reducción rápido contribuye con un $50 \%$ del total de la cantidad de $\mathrm{Fe}^{\mathrm{II}}$-Cit $\mathrm{c}$ generado en la reducción fotoinducida.

En las ecuaciones 7 y 8 se muestran la formación del estado excitado TCML en el polímero y el subsecuente quenching por TEA:

$\left\{\left[(\text { vpy })_{2}-v^{-v y R e}{ }^{I}(\mathrm{CO})_{3}(\text { tmphen })^{+}\right]\right\}_{n \sim 200}+\mathrm{hv}$

$\longrightarrow \quad\left\{\left[(\mathrm{vpy})_{2}-\mathrm{vpyRe}^{\mathrm{I}}(\mathrm{CO})_{3}(\mathrm{tmphen})^{+}{ }^{*}\right]\right\}_{\mathrm{p}}\left\{\left[(\mathrm{vpy})_{2}-\mathrm{vpyRe}(\mathrm{CO})_{3}(\text { tmphen })^{+}\right]\right\}_{\mathrm{q}}$

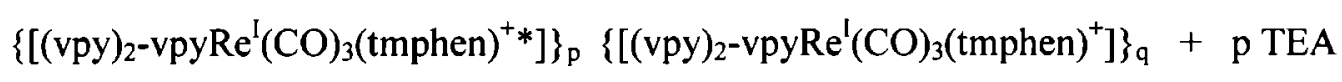

$\longrightarrow\left\{\left[(\mathrm{vpy})_{2}-\mathrm{vpyRe}^{\mathrm{I}}(\mathrm{CO})_{3}(\text { tmphen })^{\bullet}\right]\right\}_{\mathrm{p}}\left\{\left[(\mathrm{vpy})_{2}-\mathrm{vpyRe}^{\mathrm{I}}(\mathrm{CO})_{3}(\text { tmphen })^{+}\right]\right\}_{\mathrm{q}}+\mathrm{p} \mathrm{TEA}^{\bullet+}$ 
En la ecuación $7 \operatorname{Re}^{\mathrm{I}}(\mathrm{CO})_{3}(\text { tmephen })^{+*}$ denota el estado excitado de TCML del cromóforo $-\mathrm{Re}^{\mathrm{l}}(\mathrm{CO})_{3}(\text { tmephen })^{+}$. En la mezcla de solventes $\mathrm{CH}_{3} \mathrm{CN} / \mathrm{H}_{2} \mathrm{O}$, la reducción del estado excitado en el polímero de $\operatorname{Re}^{\mathrm{I}}$ produce $-\mathrm{Re}^{\mathrm{I}}(\mathrm{CO})_{3}(\mathrm{tmphen})^{\bullet}$ en concentración $\left[-\operatorname{Re}^{1}(\mathrm{CO})_{3} \text { (tmphen) }\right]_{0} \sim 2 \times 10^{-6} \mathrm{M}$ ecuación 8. Alguno de estos radicales pueden quedar cercanos a $\mathrm{Fe}^{\text {III }}$-Cit $\mathrm{c}$ en el aducto pudiendo participar en la reducción rápida de $\mathrm{Fe}^{\mathrm{III}}$-Cit $\mathrm{c}$ evidenciada por los cambios de absorbancia que pueden observarse en la figura 7. Basados en los resultados del análisis de MCVR en la figura 9 y 10 , el espectro resuelto en el tiempo de la figura 7 , puede interpretarse como el cambio en el espectro de la solución, cuando decaen los pendientes $-\operatorname{Re}^{\mathrm{I}}(\mathrm{CO})_{3}(\text { tmphen })^{*}$ y se genera la especie $\mathrm{Fe}^{\mathrm{II}}$-Cit c. Es importante destacar que la diferencia entre el coeficiente de extinción molar de $\mathrm{Fe}^{\mathrm{II}}-\mathrm{Cit} \mathrm{c}$ y Fe ${ }^{\mathrm{III}}-\mathrm{Cit} \mathrm{c}, \Delta \varepsilon\left(\mathrm{Fe}^{\mathrm{II}}-\mathrm{Fe}^{\mathrm{III}}\right)$, es un orden de magnitud mayor que el coeficiente de extinción molar de $-\operatorname{Re}^{\mathrm{I}}(\mathrm{CO})_{3}$ (tmphen), por lo tanto la contribución del radical $-\operatorname{Re}^{\mathrm{l}}(\mathrm{CO}) 3\left(\right.$ tmphen) ${ }^{*}$ a la absorción en la figura 7 es sólo relevante a longitudes de onda entre 600 y $700 \mathrm{~nm}$, donde $\Delta \varepsilon\left(\mathrm{Fe}^{\mathrm{II}}-\mathrm{Fe}^{\mathrm{III}}\right)$ es pequeño. Del análisis MVCR, es posible estimar la concentración generada en experimentos de flash fotólisis. La concentración inicial de $-\operatorname{Re}^{\mathrm{I}}(\mathrm{CO}) 3(\text { tmphen })^{*},\left(\left[-\operatorname{Re}^{\mathrm{I}}(\mathrm{CO}) 3(\text { tmphen })^{\circ}\right]_{0} \sim 5 \mathrm{x}\right.$ $10^{-6} \mathrm{M}$. La conversión máxima de $\mathrm{Fe}^{\mathrm{III}}$-Cit $\mathrm{c}$ a $\mathrm{Fe}^{\mathrm{II}}-\mathrm{Cit} \mathrm{c}$ da una concentración de $\mathrm{Fe}^{\mathrm{II}}-$ Cit c, $\left[\mathrm{Fe}^{\mathrm{II}}-\mathrm{Cit} \mathrm{c}\right]_{\max } \sim 5 \times 10^{-6} \mathrm{M}$.

La concentración inicial de $-\operatorname{Re}^{\prime}(\mathrm{CO}) 3(\text { tmphen })^{\bullet}$ generada en experimentos de flash fotólisis sobre soluciones que contienen polímero, TEA y Fe ${ }^{\mathrm{III}}-\mathrm{Cit} \mathrm{c}$ en la mezcla de solventes $\mathrm{CH}_{3} \mathrm{CN} / \mathrm{H}_{2} \mathrm{O}$ 1:1 (v:v), es dos órdenes de magnitud mas baja que la generada en $\mathrm{CH}_{3} \mathrm{CN}$; esta diferencia puede explicarse en base a dos efectos del solvente:

(i), el rendimiento cuántico del polímero de $\mathrm{Re}^{\mathrm{I}}$ es tres veces mas bajo en una mezcla de solventes $\mathrm{CH}_{3} \mathrm{CN} / \mathrm{H}_{2} \mathrm{O} 50 \%$ que en $\mathrm{CH}_{3} \mathrm{CN}$; 
(ii), la concentración de TEA decrece en la mezcla de solventes $\mathrm{CH}_{3} \mathrm{CN} / \mathrm{H}_{2} \mathrm{O}$ $50 \%$, debido a la mayor protonación.

Los radicales producidos por oxidación fotoinducida de TEA, ecuación 8 , no reaccionan con el polímero, la desaparición de los radicales TEA $^{*+}$ ocurre vía ecuación 9 en competición con la reducción lenta del $\mathrm{Fe}^{\mathrm{III}}-\mathrm{Cit}^{\mathrm{16-17}}$

$\mathrm{TEA}^{\bullet+}+\mathrm{TEA} \longrightarrow \mathrm{TEA}^{\bullet}+\mathrm{TEAH}^{+}$

$2 \mathrm{TEA}^{\bullet} \longrightarrow \mathrm{TEA}+\left(\mathrm{CH}_{3} \mathrm{CH}_{2}\right)_{2} \mathrm{~N}-\mathrm{CH}=\mathrm{CH}_{2}$

Mientras que la ecuación 9 ha sido discutida en la literatura, los cambios en el espectro observados en la figura 7 dan cuenta de la reacción entre radicales TEA con el aducto. 


\section{Referencias}

1. Qu, X.; Lu, T.; Dong, S. J. Mol. Cat. A : Chemical 1995, 102, 111.

2. Koppenol, W. H.; Rush, J. D.; Mills, J. D. and Margoliash, E. Mol. Biol. Evol. $1991,8,545$.

3. Koppenol, W. H. and Margoliash, E. J. Biol. Chem. 1982, 257, 4426.

4. (a) Margoliash, E. Proc. Natl. Acad. Sci. (USA) 1963, 50, 672; (b) Chattopadhyay, K. and Mazumdar, S. Biochemistry 2003, 42, 14606; (c) Liang, L.; Yao, P.; Jiang, M.; Zhang, G. and Yan, Y. Langmuir 2005, 21, 10662.

5. Durham, B.; Pan, L. P.; Long, J. E. and Millett, F. Biochemistry 1989, $28,8659$.

6. Liu, R.Q.; Geren, L.; Anderson, P.; Fairris, J.L.; Peffer, N.; McKee, A.; Durham, B.; Millett, F. Biochimie 1995, 77, 549.

7. Chang, I-Jy; Gray, H. B. and Winkler, J. R. J. Am. Chem. Soc. 1991, 113, 7056.

8. Pan, L. P.; Durham, B.; Wolinska, J. and Millett, F. Biochemistry 1988, 27, 7180 .

9. Kutal, C.; Weber, M.A.; Ferraudi,G. and Geiger, D. Organometallics 1985, 4, 2161.

10. Butt, W. D.; Keilin, D. _Proc. R. Soc. Lond., B 1962, 156, 429.

11. Butler, J.; Jayson, G. G. and Swallow, A. J. Biochim. Biophys. Acta 1975, 408, 215.

12. Sutin, N. and Kandell, J. K. J. Biol. Chem. 1972, 247, 6932.

13. Swanson, R.; Trus, B. L.; Mandel, N.; Mandel, G.; Kallai, O. B. and Dickerson, R. E. J. Biol. Chem. 1977, 252, 759.

14. George, P.; Glauser, S. C. and Schejter, A. J. Biol. Chem. 1967, 242, 1690.

15. Schejter, A. and Aviram, I. Biochemistry 1969, 8, 149.

16. Goez, M. and Sartorius, I. J. Am. Chem . Soc. 1993, 115, 11123.

17. Goez, M. and Sartorius, I. J. Phys. Chem. A 2003, 107, 8539. 
Capítulo IV

Reacción bimolecular de transferencia de electrones entre un polímero de $\operatorname{Re}^{\mathrm{I}} \mathrm{y}$ aminas $\mathrm{y}$ un monómero de $\operatorname{Re}^{\mathrm{I}}$ y aminas 


\section{Introducción}

Las reacciones de transferencia de electrones son de gran importancia en la Química y en casi todas las disciplinas relacionadas con ella. La transferencia de un sólo electrón ha mostrado repetidamente ser un medio común de activar moléculas orgánicas, inorgánicas y biológicas a ulteriores transformaciones químicas de una variedad muy diversa. La omnipresencia de tales reacciones ha garantizado el involucramiento de los interrogantes fundamentales de la Química moderna en la investigación de las mismas.

El estudio de las reacciones de transferencia de electrones en sistemas químicos bien definidos ha jugado un papel importante en la investigación de los mecanismos de las reacciones químicas. Efectivamente, en la comprensión del proceso de transferencia de electrones subyace una base conceptual afirmada en la Física teórica y al mismo tiempo este proceso resulta un paso de importancia primordial en procesos biológicos tan complicados como la respiración y la fotosíntesis. El estudio de los procesos de transferencia de electrones en la Química puede proveer datos que permitan confirmar la validez de las teorías existentes y al mismo tiempo permite construir modelos para las reacciones de transferencia de electrones en sistemas biológicos.

La creencia de que la velocidad de la transferencia de electrones en las reacciones de óxido-reducción de los complejos metálicos era inmensurablemente rápida fue descartada después de la Segunda Guerra Mundial mediante el uso de trazadores radioactivos, técnicas de flujo, y métodos basados en la Resonancia Magnética Nuclear y en la Resonancia Paramagnética electrónica. El conocimiento actual de las reacciones de transferencia de electrones se basa en los grandes avances que se produjeron en el tema hacia fines de la década del cincuenta y durante la década 
del sesenta. Una de las razones de este avance fue experimental, en donde Henry Taube ${ }^{1}$ y sus colaboradores jugaron un rol primordial. Mostraron la importancia de la estructura primaria en la determinación de las diferentes rutas que puede seguir la transferencia de electrones (reacciones de esfera interna y reacciones de esfera externa) e investigaron el papel que juega el ligando-puente en la determinación de las constantes de velocidad para las reacciones de transferencia de electrones ${ }^{1,2}$ entre los isómeros de valencia con dos centros metálicos. Paralelamente Marcus ${ }^{3}$, Hush $^{4}$, y Levich, Dogonadze y colaboradores ${ }^{5}$ realizaron avances importantes en la Teoría de la transferencia de electrones.

Un aspecto esencial del problema de la transferencia de electrones es el cambio resultante en la configuración nuclear de equilibrio de un ión o molécula cuando gana o pierde un electrón. En el caso de un complejo metálico en un solvente polar, este cambio de configuración involucra cambios en los ángulos y distancias de enlace metal-ligando e intraligando así como cambios en las vibraciones y en las orientaciones de los dipolos del solvente que rodean al complejo. Libby mencionó por primera vez en $1952^{6}$ que el acoplamiento de la transferencia de electrones a aquellas configuraciones nucleares está gobernado por los requerimientos de conservación del momento y la energía expresados en el Principio de Franck-Condon. De acuerdo con este principio, las distancias internucleares y las velocidades nucleares no cambian durante una transición electrónica, la transición electrónica ocurre con la constancia de la configuración y el momento. Este es un requerimiento central tanto para las teorías clásicas como para las teorías cuánticas de transferencia de electrones.

En las teorías clásicas se hace uso de un formalismo de complejo-activado en el cual la transferencia de electrones ocurre en la intersección de dos superficies de energía 
potencial (una para los reactivos y una para los productos). El Principio de FranckCondon se obedece debido a que las configuraciones nucleares y las energías de los productos y los reactivos son las mismas en la intersección. Por otro lado, en las teorías cuánticas la intersección de las superficies de energía potencial es menos enfatizada, se permite el efecto túnel para el paso del estado inicial al estado final, y la transferencia de electrones es tratada como una transición no radiativa entre los estados de los reactivos y los productos. La idoneidad de una configuración nuclear particular para la transferencia de electrones se relaciona con el cuadrado de la integral de solapamiento de las funciones de onda vibracionales de los reactivos y los productos.

Las primeras teorías de transferencia de electrones fueron no-adiabáticas ${ }^{\mathbf{6 , 7 8 , 9}}$; esto es, la constante de velocidad dependía explicitamente de la interacción electrónica de los reactivos, y el cambio de la configuración nuclear no era calculado específicamente. ${ }^{7,9}$ Unos años después Marcus publicó el primero de una serie de trabajos sobre la teoría clásica (complejo-activado) de transferencia de electrones. En los trabajos de Marcus ${ }^{\mathbf{1 0 , 1 1 , 1 2}}$ se suponía que la transferencia de electrones era adiabática; esto es, se suponía que la interacción electrónica era lo suficientemente grande como para no aparecer en la expresión de la constante de velocidad. Los cambios de la configuración intramolecular eran despreciados y los cambios en la configuración del solvente eran tratados clásicamente, utilizando conceptos desarrollados en la discusión de los espectros de transferencia de carga ${ }^{13,14}$. Este modelo no tenía en cuenta el hecho importante, ya establecido en esa época, de que a pesar de la similitud de los cambios de configuración del solvente en las reacciones de intercambio de electrones entre los iones +2 y +3 las velocidades variaban en varios órdenes de magnitud. Este resultado indicaba 
claramente la importancia de las configuraciones electrónicas detalladas de los reactivos y los productos en la determinación de la velocidad de transferencia de electrones.

En 1957 Marcus $^{15}$ incluyó los cambios de configuración intramolecular en los cálculos de las velocidades de transferencia de electrones de las reacciones de intercambio. Este tratamiento fue luego generalizado por Marcus y Hush para incluir cambios netos de energía libre. En 1962 se introdujo un formalismo semi-clásico en el cual la expresión clásica para el cambio de la configuración intramolecular era corregida por el efecto tunel. ${ }^{16}$ Paralelamente a estos desarrollos, Levich ${ }^{5 e, 17,18}$ y Dogonazde $e^{19,20}$ publicaron una descripción mecano-cuántica de los cambios de la configuración del solvente en donde se utilizaba la Teoría de perturbaciones de primer orden dependiente del tiempo para describir la evolución temporal de los estados de orden cero iniciales. En los años setenta varios aspectos mecano-cuánticos del problema de la transferencia de electrones fueron tratados en detalle ${ }^{21-30}$ y se realizaron cálculos ab-initio de orbitales moleculares para determinar las velocidades de las reacciones de intercambio. ${ }^{31,32} \mathrm{Se}$ lograron importantes avances en la aplicación del formalismo de la transición no radiativa a los procesos de transferencia de electrones, llegándose a un muy elegante tratamiento en el cual el donor de electrones, el aceptor y las moléculas de solvente circundantes se trataron como una supermolécula ${ }^{\mathbf{3 3}}$. Al mismo tiempo, una creciente cantidad de trabajos experimentales indicaron ${ }^{34-38}$ la necesidad de revisiones al tratamiento clásico (y mecano-cuántico) de las reacciones altamente exotérmicas. A pesar de que ha habido mucha discusión sobre este problema y se han realizado progresos $^{24,33,39-42}$ todavía existen varios interrogantes. Un defecto importante del tratamiento mecano-cuántico en su forma actual es que no da cuenta de entropías de 
reacción altas. Para solucionar este problema generalmente se usan las expresiones de Marcus para la energía libre de reorganización del solvente.

A pesar de los defectos de las teorías en la región altamente exoergónica, hay un acuerdo general en lo concerniente al tratamiento de las reacciones de transferencia de carga termoneutrales y moderadamente exoergónicas. .

En este capitulo de la tesis, se muestran los resultados de la desactivación no radiativa del estado excitado de TCML en el polímero $\left\{(\mathrm{vpy})_{2} \mathrm{vpyRe}(\mathrm{CO})_{3}\left(2,2^{\prime} \mathrm{Bpy}\right)^{+}\right\}_{\mathrm{n} \sim 200}, \mathrm{y}$ del estado excitado de TCML en el monómero PyRe $(\mathrm{CO})_{3}\left(2,2^{\prime} \mathrm{Bpy}\right)^{+}$, por reacción con aminas alifáticas y aromáticas.

Además, se presentan los resultados de reducción fotoquímica y por radiólisis de pulso del polímero $\left\{(\mathrm{vpy})_{2} \mathrm{vpyRe}(\mathrm{CO})_{3}(\mathrm{bpy})^{+}\right\}_{n \sim 200}$.

Debido a que la reacción de desactivación no radiativa del estado excitado de TCML implica una transferencia de electrones, en la primera parte del capítulo se hará una breve introducción a conceptos básicos para dicha transferencia. 


\section{Conceptos teóricos}

\section{i. Transferencia de electrones fotoinducida}

La absorción de luz a nivel molecular necesariamente produce un cambio en la estructura electrónica. Si el estado excitado electrónico resultante vive lo suficiente como para estar en equilibrio térmico con sus alrededores, posee su propio conjunto de niveles vibracionales característicos y por lo tanto sus propiedades termodinámicas, espectrales y de reactividad química. Todo estado electrónico excitado posee sus propiedades termodinámicas de óxido-reducción aumentadas cuando se las compara con el estado fundamental de la molécula. Estas propiedades redox aumentadas son una consecuencia del acto de excitación en el cual un electrón es promovido a un estado electrónico superior creándose un sitio reductor y simultáneamente un sitio oxidante en el hueco creado por la excitación.

La sugerencia de una reactividad redox acrecentada en los estados excitados de las moléculas orgánicas fue confirmada por los notables ejemplos de los tempranos trabajos de Rehm y Weller ${ }^{38,43}$ y los trabajos de Mataga y sus colaboradores. ${ }^{44}$ Para los complejos metálicos, Gafney y Adamson sugirieron por primera vez que ocurría la transferencia de electrones de estado excitado (transferencia de electrones foto-inducida) en la desactivación del estado excitado del $\mathrm{Ru}\left(2,2^{\prime} \mathrm{Bpy}\right)_{3}{ }^{2+}$ por una serie de amino complejos de $\mathrm{Co}(\mathrm{III})$ generándose el $\mathrm{Ru}\left(2,2^{\prime} \mathrm{Bpy}\right) 3_{3}{ }^{3+}$ y $\mathrm{Co}(\mathrm{II})$ como productos de reacción. ${ }^{45}$ Experimentos posteriores de flash fotólisis mostraron que tanto el $\left[\mathrm{Ru}\left(2,2^{\prime} \mathrm{Bpy}\right)_{3}{ }^{2+}\right]^{*}$ como otros estados excitados relacionados pueden experimentar fácilmente tanto desactivación oxidativa como reductiva. ${ }^{46-51}$ 


\section{ii. Consideraciones Generales}

Las reacciones de transferencia de electrones que involucran estados excitados son de las reacciones bimoleculares más simples. En estos procesos, ecuación 1, no ocurre ruptura ni formación de un nuevo enlace y solo se transfiere un electrón entre el reactivo y el producto.

$$
\mathrm{A}^{*}+\mathrm{D} \rightarrow\left[\mathrm{A}^{*}\right]^{\bullet-}+\mathrm{D}^{\bullet+}
$$

A representa la molécula cuyo estado excitado es aceptor de un electrón y D representa la molécula donora del electrón. La constante de velocidad $\mathrm{k}_{\mathrm{q}}$ de este proceso bimolecular está controlada por varios factores. Para dilucidar estos factores, se debe considerar un mecanismo de reacción detallado. Utilizando como ejemplo la reacción de transferencia de electrones de estado excitado reductiva, ecuación 1 , la velocidad de reacción puede ser interpretada sobre la base del mecanismo mostrado en el siguiente esquema:

$$
\mathrm{A}^{*}+\mathrm{D} \underset{\mathrm{k}_{-\mathrm{d}}}{\stackrel{\mathrm{k}_{\mathrm{d}}}{\rightleftarrows}} \mathrm{A}^{*}--\mathrm{D} \underset{\mathrm{k}_{-\mathrm{e}}}{\stackrel{\mathrm{k}_{\mathrm{e}}}{\rightleftarrows}} \mathrm{A}^{-}-\mathrm{D}^{+} \stackrel{\mathrm{k}_{\mathrm{p}}}{\longrightarrow} \mathrm{A}+\mathrm{D}^{+}
$$

donde $k_{d}$ es constante de velocidad difusional, $k_{-d}$ es la constante de velocidad para la disociación del complejo de encuentro, $\mathrm{k}_{\mathrm{e}} \mathrm{y} \mathrm{k}_{\mathrm{e}}$ son las constantes de velocidad para la transferencia de electrones directa e inversa y $k_{p}$ es la constante de velocidad para la 
disociación del par iónico y/ó la recombinación de cargas. Este mecanismo puede ser resuelto aplicando la hipótesis del estado estacionario:

$$
\begin{aligned}
& d\left[A^{*}--D\right] / d t=k_{d}\left[A^{*}\right][D]+k_{-e}\left[A^{-}--D^{+}\right]-k_{-d}\left[A^{*}--D\right]-k_{e}\left[A^{*}--D\right]=0 \\
& d\left[A^{*}--D^{+}\right] / d t=k_{e}\left[A^{*--D}\right]-k_{-e}\left[A^{-}--D^{+}\right]-k_{p}\left[A^{-}-D^{+}\right]=0
\end{aligned}
$$

de donde se pueden obtener las expresiones para las concentraciones de $\left[\mathrm{A}^{*}-\mathrm{-D}\right]$ y $\left[\mathrm{A}^{*}-\right.$ $\mathrm{D}^{+}$] de estado estacionario:

$$
\begin{aligned}
& {\left[A^{*}--D\right]_{e e}=\frac{k_{d}\left[A^{*}\right][D]+k_{-e}\left[A^{-}--D^{+}\right]_{e e}}{k_{e}+k_{-d}}} \\
& {\left[A^{-}--D^{+}\right]_{e e}=\frac{k_{e}\left[A^{*}--D\right]_{e e}}{k_{p}+k_{-e}}}
\end{aligned}
$$

Reemplazando $\left[\mathrm{A}^{-}--\mathrm{D}^{+}\right]_{\mathrm{ee}}$ en la expresión para $\left[\mathrm{A}^{*}-\mathrm{-D}\right]_{\mathrm{ee}} \mathrm{y}$ reordenando obtenemos:

$$
\left[\mathrm{A}^{*}--D\right]_{e \mathrm{e}}=\frac{\mathrm{k}_{\mathrm{d}}\left(\mathrm{k}_{\mathrm{p}}+\mathrm{k}_{-\mathrm{e}}\right)\left[\mathrm{A}^{*}\right][\mathrm{D}]}{\mathrm{k}_{\mathrm{p}} \mathrm{k}_{\mathrm{e}}+\mathrm{k}_{\mathrm{p}} \mathrm{k}_{-\mathrm{d}}+\mathrm{k}_{-\mathrm{e}} \mathrm{k}_{-\mathrm{d}}}
$$

Si escribimos la ecuación de velocidad para la desaparición de $A^{*}$ obtenemos:

$$
-d\left[A^{*}\right] / d t=k_{d}\left[A^{*}\right][D]-k_{-d}\left[A^{*--} D\right]=k_{q}\left[A^{*}\right][D]
$$


Reemplazando $\left[\mathrm{A}^{*}--\mathrm{D}\right]_{\mathrm{ee}}$ en la ecuación 7 y reordenando se obtiene

$$
k_{q}=\frac{k_{d}}{1+\frac{k_{-d}}{k_{e}}+\frac{k_{e} k_{-d}}{k_{e} k_{p}}}
$$

Bajo la suposición de que $\mathrm{k}_{\mathrm{p}} \gg \mathrm{k}_{-\mathrm{e}}$ la constante de desactivación global $\mathrm{k}_{\mathrm{q}}$ puede expresarse como:

$$
\mathrm{k}_{\mathrm{q}}=\frac{\mathrm{k}_{\mathrm{d}}}{1+\frac{\mathrm{k}_{-\mathrm{d}}}{\mathrm{k}_{\mathrm{e}}}}
$$




\section{iii. Aspectos Termodinámicos}

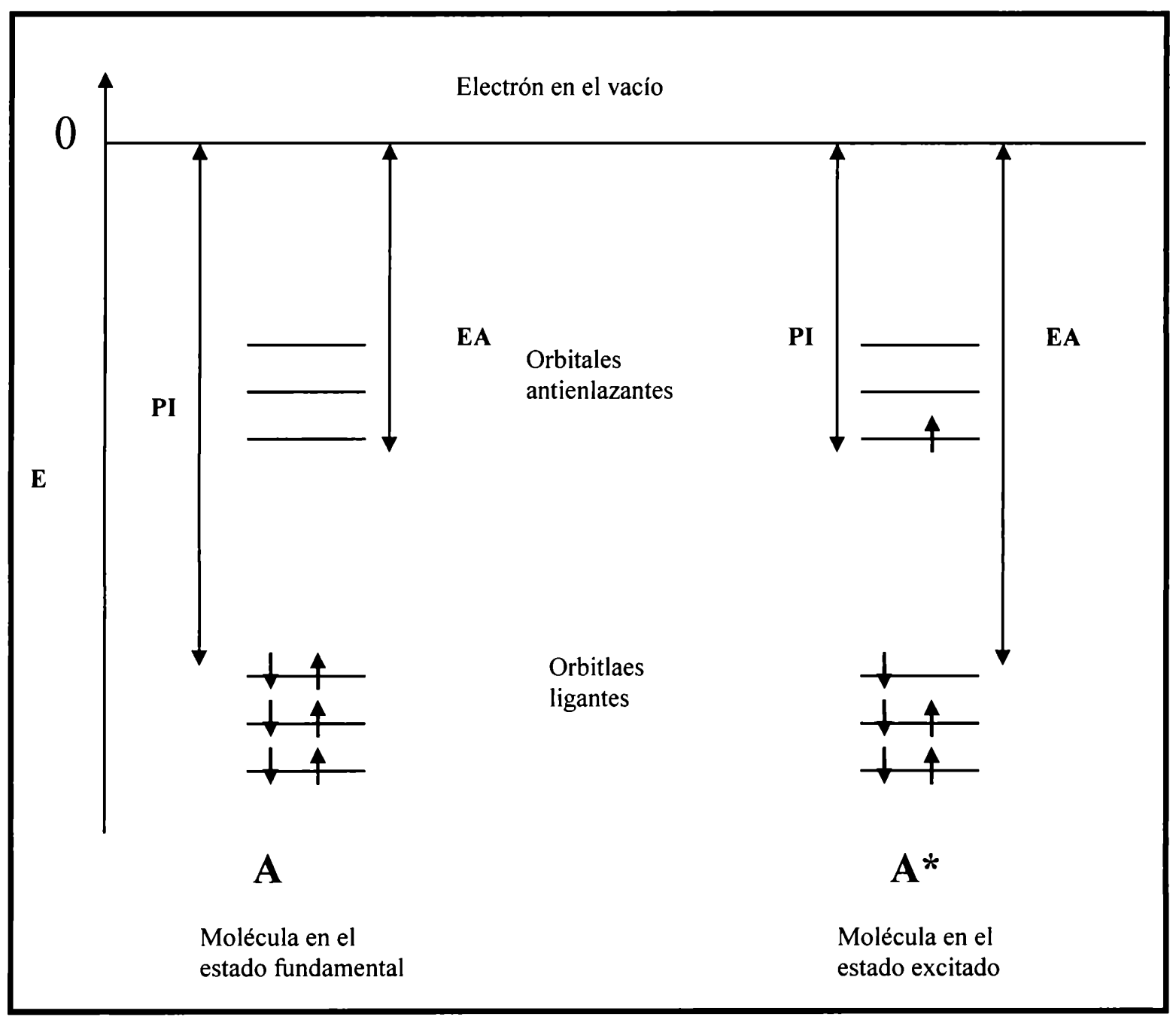

Figura 2: Representación orbital de procesos de óxido-reducción para el estado fundamental y excitado

Las energías asociadas a la figura 2 se refieren a la fase gaseosa, donde, afinidad electrónica y potencial de ionización, son aplicables. Para una simple reacción de 
transferencia de electrones en fase gaseosa, el cambio en energía libre para el intercambio de electrón en el estado fundamental está dado por, $\Delta \mathrm{G}_{\mathrm{te}, \mathrm{ef}}$

$$
\begin{aligned}
& \mathrm{D}+\mathrm{A} \longrightarrow \mathrm{D}^{+\bullet}+\mathrm{A}^{\bullet} \\
& \Delta \mathrm{G}_{\mathrm{te}, \mathrm{ef}}=(\mathrm{PI})_{\mathrm{D}}-(\mathrm{EA})_{\mathrm{A}}
\end{aligned}
$$

Para el estado excitado del donor, $D^{*}$, el valor de $\Delta \mathrm{G}$ difiere en la magnitud de energía de excitación electrónica, $\mathrm{E}_{\mathrm{D}^{*}}$. Por lo tanto para la reacciones 13 y 14, deben utilizarse las ecuaciones 15 y 16 . Las cuales toman en cuenta la energía de excitación.

$$
\mathrm{D}^{*}+\mathrm{A} \longrightarrow \mathrm{D}^{+\bullet}+\mathrm{A}^{-\bullet}
$$

ó

$$
\begin{aligned}
& \mathrm{D}^{*}+\mathrm{A} \longrightarrow \mathrm{D}^{-\bullet}+\mathrm{A}^{+\bullet} \\
& \Delta \mathrm{G}_{\mathrm{te}, \mathrm{ef}}=(\mathrm{PI})_{\mathrm{D}}-(\mathrm{EA})_{\mathrm{A}}-\mathrm{E}_{\mathrm{D}^{*}} \\
& \Delta \mathrm{G}_{\mathrm{te}, \mathrm{ef}}=(\mathrm{PI})_{\mathrm{A}}-(\mathrm{EA})_{\mathrm{D}}-\mathrm{E}_{\mathrm{D}^{*}}
\end{aligned}
$$

Estas ecuaciones muestran como la transferencia de electrones es mas favorable en el estado excitado $D^{*}$ que en el estado fundamental. 
Para determinar la energética asociada a una reacción en solución, hay al menos dos opciones.

1) Se puede calcular $\Delta \mathrm{G}$ para le reacción en fase gaseosa, y luego corregir con la energía de solvatación de todos los participantes de la reacción $\left(D^{*}, A^{*}, D, A, D^{+\bullet}\right.$ y

$\left.A^{-\bullet}\right)$

2) Es posible utilizar los potenciales electroquímicos de oxidación y reducción involucrados en el cálculo de cambio de energía libre directamente en solución.

Si bien ambas opciones son válidas, desde el punto de vista experimental, algunos parámetros requeridos para la opción 1) son frecuentemente inaccesibles. Por ejemplo la energía de disolución de $\mathrm{D}^{*}$ y $\mathrm{D}^{+\bullet}$ (de la fase gaseosa a la fase solución) no son accesibles experimentalmente. En cambio, para la opción 2) los parámetros electroquímicos son frecuentemente accesibles o pueden ser determinados usando técnicas electroquímicas estándar como voltametría cíclica, y es la opción más utilizada.

Por lo tanto, utilizando la opción 2), en solución el $\Delta \mathrm{G}$ está dado por:

$$
\Delta G^{0} \approx E_{D^{+} / D}^{0}-E_{A / A^{-}}^{0}-E_{D^{*}}
$$

donde, $E_{\left(D^{+} / D\right)}^{0}$ y $E_{\left(A / A^{-}\right.}^{0}$ son los potenciales electroquímicos correspondientes para A y $\mathrm{D}$, ambos expresados como reducción. El símbolo $\approx$ hace énfasis en que la ecuación es sólo una aproximación.

Son requeridas dos aproximaciones para el análisis de reacciones fotoquímicas que involucran transferencia de carga y son: 
1) El término $E_{D^{*}}$, es una entalpía no una energía libre. La diferencia es $-T \Delta S_{D^{*}}$ donde $\Delta \mathrm{S}_{\mathrm{D}^{*}}$ es la entropía de excitación de $\mathrm{D}$. Mientras este término entrópico sea despreciable, la aproximación es válida.

2) Hay una energía coulómbica asociada a la aparición de dos partículas con carga opuesta, ecuación 12 donde dos moléculas neutras se transforman en un anión y un catión, el término de corrección coulómbico es proporcional a, $-\mathrm{e}^{2} / \varepsilon r$, donde e es la carga del electrón, $\varepsilon$ es la constante dieléctrica del solvente y $\mathrm{r}$ es la distancia aproximada entre $\mathrm{D}^{+\bullet} \mathrm{y}^{-\bullet}$, que en una primera aproximación puede ser tomada como la suma de los radios de los iones.

El término coulómbico será pequeño (comparado con $\mathrm{E}_{\mathrm{D}^{*}}$ ) en un solvente polar. Por

ejemplo en agua, el término coulómbico es menor a $1 \mathrm{Kcal} / \mathrm{mol}$ a una separación de 2 $\AA$ En contraste, en un solvente no polar el término puede ser lo suficientemente grande a favor de la asociación iónica mas que de la disociación.

\section{iv. Teoría de Marcus para la transferencia de electrones}

En un principio, como ya se mencionó, la transferencia de electrones ha sido considerada como la reacción química más simple, ya que no hay ruptura ni formación de enlaces. Se puede considerar sólo como el pasaje de un electrón de un orbital a otro.

Una de las primeras investigaciones sobre este tema, fueron realizadas por Libby $^{6}, 1952$, pero esta no tiene en cuenta la importancia de la reorganización del solvente en el control de la velocidad de la reacción de transferencia en el estado 
fundamental. Por ejemplo, cuando una molécula, donora de electrón, D (solvatada) se convierte en $\mathrm{D}^{+\bullet}$ (solvatado) la esfera de solvatación de $\mathrm{D}$ y $\mathrm{D}^{+\bullet}$ debe cambiar, reflejando la reorganización de moléculas de solvente (dipolos) alrededor de la carga eléctrica naciente, una situación similar se da en los alrededores del aceptor de electrones.

Las suposiciones de Libby fueron que la transferencia de electrones sería un proceso mucho mas rápido que la unión molecular y vibracional o la reorganización del solvente, postuló entonces, que los cambios estructurales ocurren luego de la transferencia de electrones.

En cambio Marcus propuso una nueva idea ya que si la reorganización molecular y del solvente ocurren luego de la transferencia de energía, entonces los requerimientos energéticos corresponden a una transición vertical ( $\lambda$, figura 3$)$. Por lo tanto, la teoría de Marcus propone, que para que la transferencia de energía ocurra las moléculas y el solvente deben estar reorganizados. Una vez que la reorganización del solvente y las moléculas toman lugar, la transferencia electrónica es rápida.

Estas reorganizaciones del solvente y los reactantes, puede ser dividida en dos tipos, un término de reorganización de esfera interna y un término de reorganización de esfera externa. Estos términos, derivan de los trabajos de Libby sobre iones metálicos para los cuales esfera interna hace referencia a los ligandos directamente unidos al ión metálico y esfera externa se refiere a las moléculas de solvente que solvatan al complejo. En la teoría de Marcus, el término esfera interna se refiere a las coordenadas internas de reactantes y productos (longitud de unión y ángulos), mientras que esfera 
externa se refiere a las moléculas del solvente en los alrededores de reactantes y productos.

Basado en la idea de esfera interna y esfera externa, Marcus desarrolló un modelo para la transferencia de electrones, el cual permite el cálculo de la energía asociada con la esfera interna y externa.

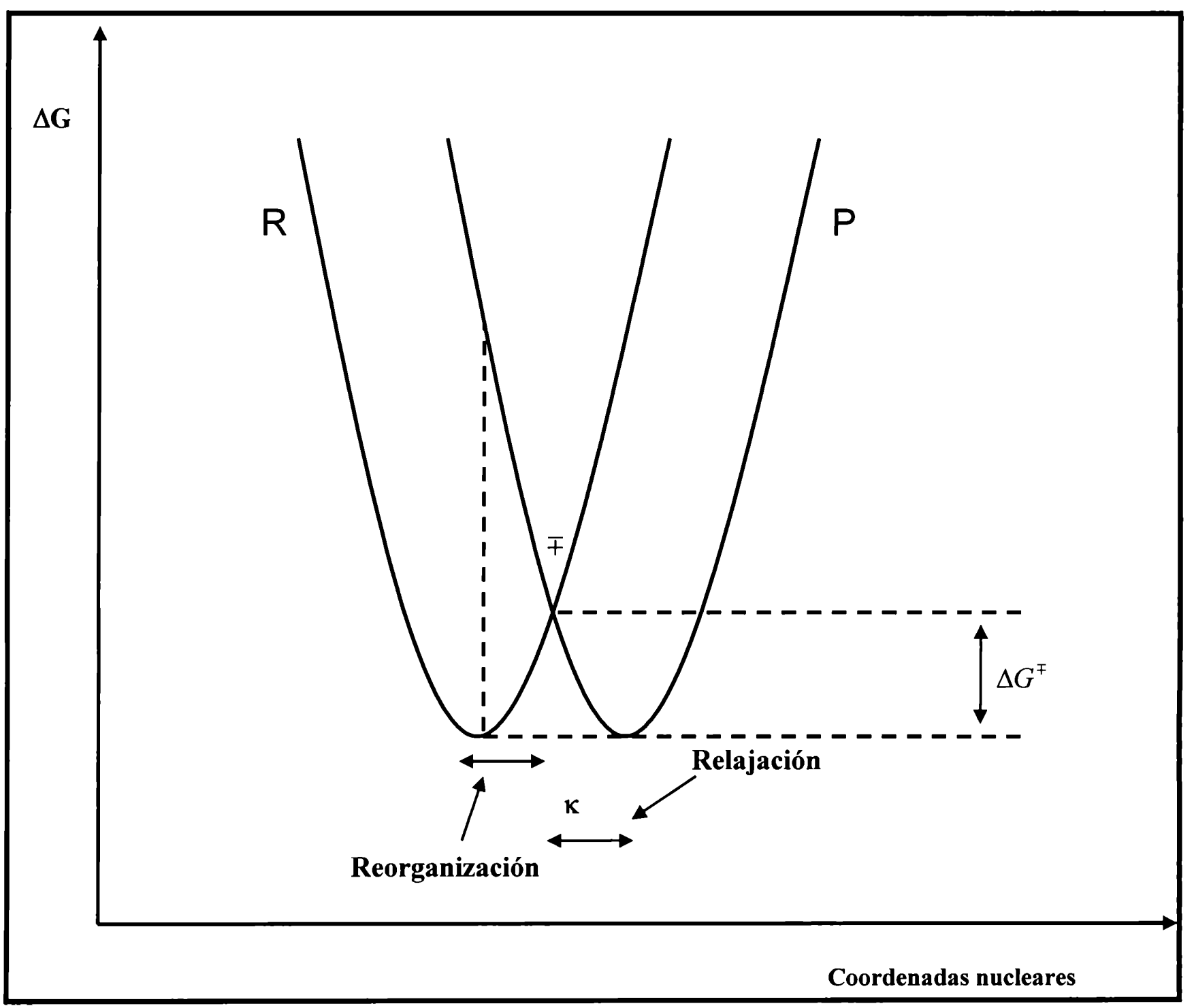

Figura 3: Descripción de la energía potencial de la reacción de transferencia de electrones con $\Delta \mathrm{G}^{0}=0$. El punto $\mp$ representa el estado de transición. 
Una premisa básica de la teoría de Marcus es que sólo es necesaria una débil interacción electrónica entre reactantes y productos para que ocurra un simple proceso de transferencia de electrones. La teoría de Marcus relaciona la transferencia de electrones con conceptos como, teoría de velocidad de reacción, superficie de energía potencial y solvatación de iones. Por ejemplo es posible comenzar con la conocida ecuación de Arrhenius que relaciona la constante de velocidad de la transferencia de electrones con una energía de activación y un factor de frecuencia

$$
k_{e t}=v_{N} \kappa \exp \left(-\Delta G^{\mp} / R T\right)
$$

Donde, $\Delta G^{\mp}$ es la energía libre de activación de la transferencia de electrones, $v_{N}$ es un factor electrónico y $\kappa$ es el coeficiente de transmisión: la probabilidad de que los reactantes, una vez alcanzado algún estado crítico de esfera externa coordinada, sean capaces de dar productos. El significado de algunos términos de la ecuación pueden ser visualizados para la reacción con $\Delta \mathrm{G}^{0}=0$ (figura 3), en la cual la curva de energía potencial de los reactantes está representada por la parábola sobre la izquierda y la curva de energía potencial para los productos está representada por la parábola de la derecha, admitiendo las curvas de energía potencial como parábolas de un oscilador armónico. Esta aproximación de las curvas de energía como parábolas, presenta un poderoso uso de la geometría para dar algunas predicciones de la constante de la reacción de transferencia de electrones como una función de la energía de reorganización de esfera externa e interna involucradas en la reacción. 
En la figura 3, $\Delta G^{\mp}$ representa el requerimiento de energía libre para alcanzar el estado de transición, $E T^{\mp}$, desde el mínimo de la curva de energía potencial. Los reactantes, $\mathrm{R}$, necesitan reorganizarse, tanto interna como externamente para alcanzar los requerimientos que hacen posible la transferencia de electrones. Una vez alcanzado el estado de transición, $E T^{\mp}$, algunos sistemas proceden hacia la superficie de los productos mientras que otros serán "reflejados" retornando al punto de partida de los reactantes. El coeficiente de transmisión que da la probabilidad de que ha sido alcanzado el requerimiento de reorganización, será seguida del movimiento a lo largo de la superficie potencial de los productos y la correspondiente relajación.

La curva de energía potencial parabólica de los reactantes presenta una intersección con la curva de energía potencial parabólica de los productos. Las curvas de energía potencial representan la energía libre de equilibrio de los reactivos y productos para una reacción térmicamente neutra, como una función de algunas coordenadas para la reorganización del solvente. Es fácil imaginar que las coordenadas de reacción también incluyen las coordenadas de reorganización de esfera interna. Si se supone que el electrón "salta" desde R a P sin reorganización del solvente, representativamente, es como mover un punto desde el mínimo de la curva $R$ a un punto en la curva $P$, verticalmente. Para una reacción térmicamente neutra el mínimo de $\mathrm{R}$ y $\mathrm{P}$ tienen la misma energía libre, el requerimiento energético para el salto vertical de un electrón de $\mathrm{R}$ a $\mathrm{P}$ está definido con $\lambda$ y es igual a la energía de reorganización requerida para que ocurra la transferencia de un electrón. $\lambda$ corresponde a la separación vertical, entre la curva de $R$ y $P$ para una reacción isoergónica, $\Delta G^{0}=0 . \quad \lambda$ es entonces la barrera energética que debe sobrepasarse para la transferencia de energía sin ninguna 
reorganización previa; este es el modelo original de Libby para la transferencia de energía. El precio energético a pagar según la teoría de Marcus es mucho menor y se ve representativamente como la intersección de las dos superficies en la figura 4.

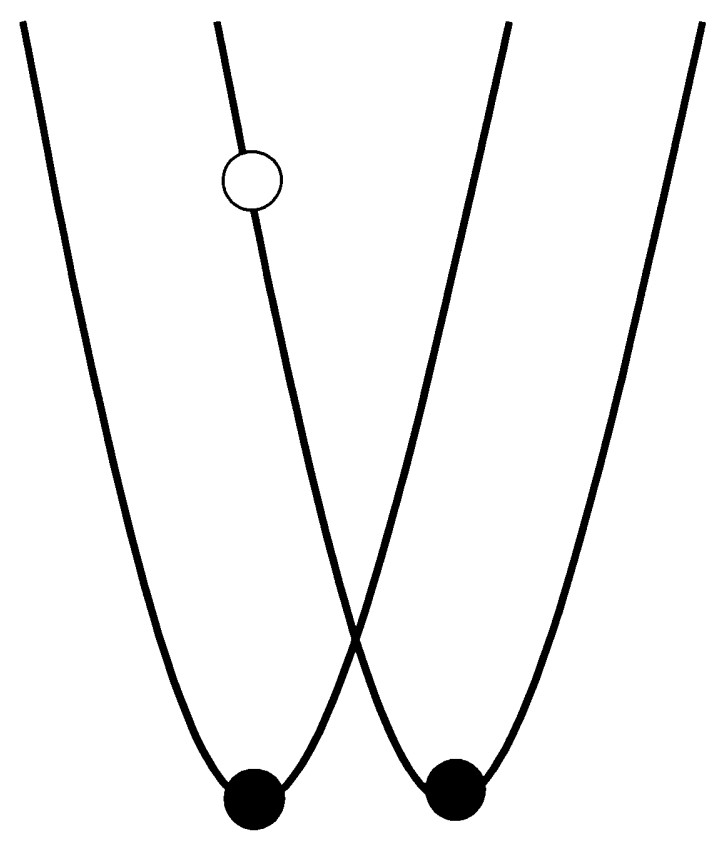

Modelo de Libby

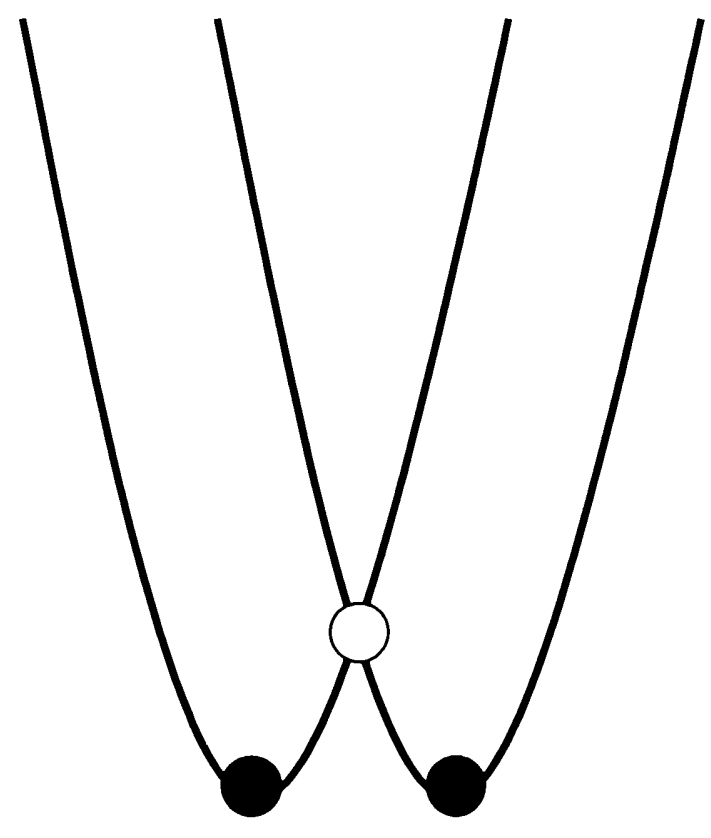

Modelo de Marcus

Estado inicial

Estado de transición

Estado final

Figura 4: Comparación de los requerimientos energéticos para la transferencia de electrones entre el modelo de Libby y el modelo de Marcus. 
El parámetro $\lambda$ está definido con referencia a la situación isoergónica, aún para reacciones donde $\Delta \mathrm{G}^{0} \neq 0$.

Cuanto mas exoergónica es una reacción, el valor de $\Delta G^{\mp}$ cambia como un reflejo del desplazamiento vertical de las curvas de los reactivos y los productos, la figura 5 muestra la evolución de las curvas de energía potencial cuando ocurre este desplazamiento.

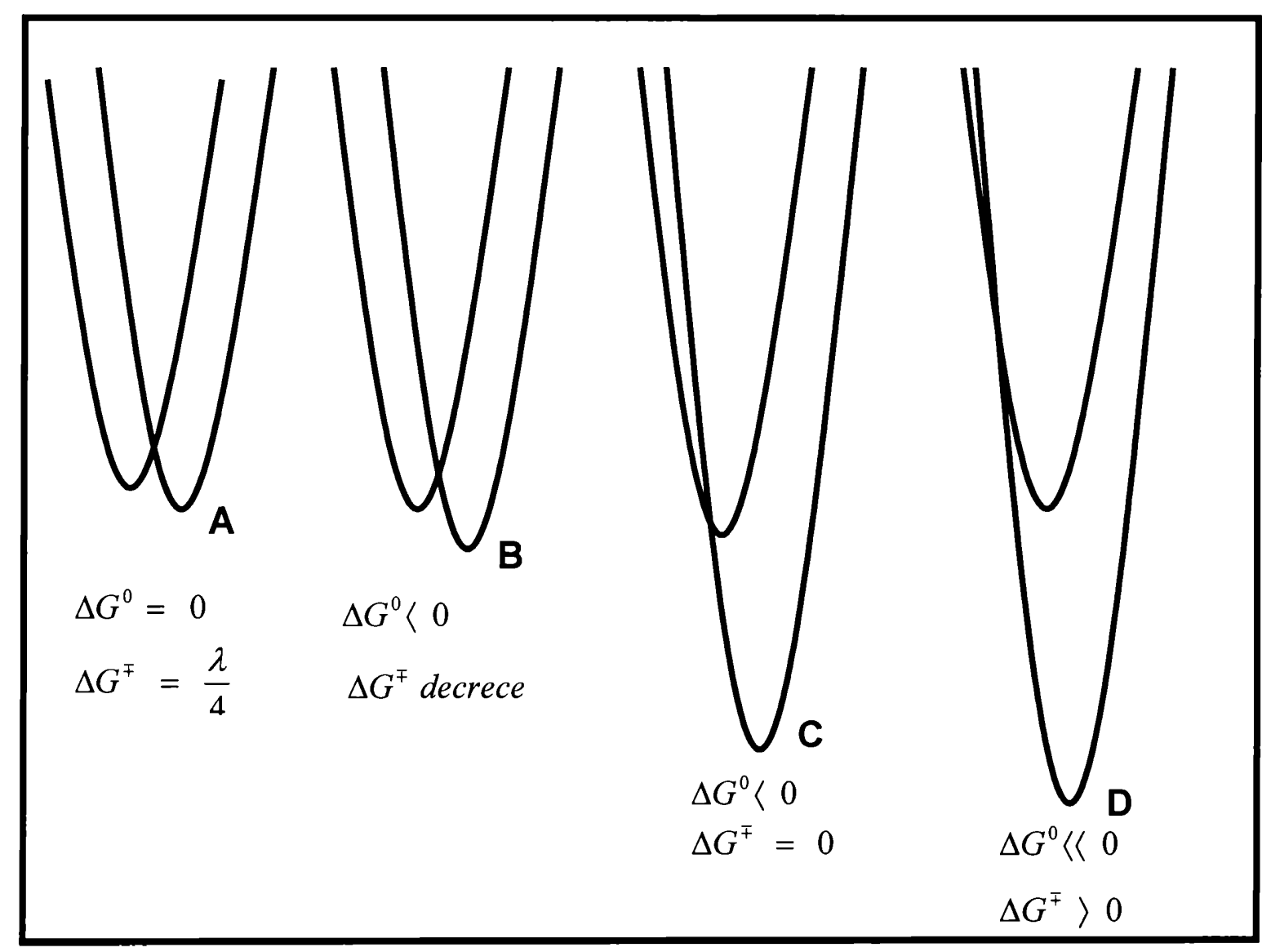

Figura 5: Esquema del movimiento de la curva de energía libre del producto $(P)$ hacia abajo $\left(\Delta \mathrm{G}^{0}\right.$ más negativa) la energía de activación primero decrece, luego es 0 , en $\mathrm{C}$ cuando la intersección coincide con el mínimo en los reactivos y eventualmente la energía de activación se incrementa con $\Delta \mathrm{G}^{0}$ más negativa, esta es la región invertida de Marcus. 
Considerando que la coordenada de reacción no cambia significativamente con la exotermicidad de la reacción la figura 5 puede interpretarse como sigue:

A-B) Primero la barrera para la transferencia de electrones $(\lambda)$ decrece cuando la reacción se vuelve mas exotérmica.

C) Luego, la curva de potencial de los productos intersecta al mínimo de la curva de los reactivos.

D) La reacción se vuelve más exotérmica que $\mathrm{C}$, existe una barrera para la transferencia de energía y se incrementa monotónicamente con el incremento en la exotermicidad.

Es decir, la energía libre de activación, $\Delta G^{\mp}$ decrece inicialmente con $\Delta \mathrm{G}^{0}$, siendo cada vez mas negativa, hasta que la curva del producto intersecta el mínimo de la curva del reactivo, figura $5 \mathrm{C}$. En la región A-C la velocidad se incrementa cuando $\Delta \mathrm{G}^{0}$ decrece, como es de esperar intuitivamente. Sin embargo, pasado este punto, figura 5D, $\Delta G^{\mp}$ se incrementa cuando $\Delta \mathrm{G}^{0}$ se vuelve más negativo. Este simple esquema predice que la constante de velocidad de transferencia de un electrón decrece cuanto mas favorable es energéticamente, figura 5 C-D, ésta es conocida como la región invertida de Marcus.

Las conclusiones hechas del análisis de la figura 5 pueden ser cuantificadas en la teoría de Marcus.

Suponiendo que las curvas de energía potencial se comportan como parábolas, para los reactivos y los productos, Marcus fue capaz de relacionar la energía de reorganización, $\lambda$, y la energía libre de reacción por medio de la ecuación: 


$$
\Delta G^{\mp}=\frac{\lambda}{4}\left(1+\frac{\Delta G^{0}}{\lambda}\right)^{2}
$$

Para una reacción isoergónica la ecuación de reduce a:

$$
\Delta G^{\mp}=\frac{\lambda}{4} \text { para } \quad \Delta G^{0}=0
$$

La condición termo-neutral $\left(\Delta G^{0}=0\right)$ permite la definición de $\lambda$, la energía de reorganización en términos de la intersección de dos parábolas. Marcus utiliza la geometría en el plano para conectar $\lambda$ con la constante de transferencia de energía experimental (la cual está relacionada con $\Delta G^{\mp}$ por la expresión de Arrhenius) y luego relacionar $\Delta G^{\mp} \operatorname{con} \lambda$.

La energía de reorganización, $\lambda$, está determinada por dos contribuciones, una de esfera externa y otra de esfera interna.

$$
\lambda=\lambda_{\text {esfera externa }}+\lambda_{\text {esfera int erna }}
$$

Ambas contribuciones pueden ser evaluadas independientemente si hay suficiente información del sistema.

El valor de $\lambda_{\text {esfera int ema }}$ reflejará los cambios en la estructura de los reactivos y productos. Examinando las estructuras de reactivos y productos, es posible tener una 
idea intuitiva de la magnitud de $\lambda_{\text {esfera int erna }}$. Este parámetro puede ser evaluado por la siguiente ecuación:

$$
\lambda_{\text {esfera int erma }}=\sum_{i}\left(\frac{f_{i}^{R} f_{i}^{R}}{f_{i}^{R}+f_{i}^{P}}\right) \Delta q_{i}
$$

Donde $\Delta q_{i}$ es el cambio en la distancia interatómica y $f_{i}$ es constante de fuerza para la i-ésima vibración. Valores típicos de $\lambda_{\text {esfera int erna }}$ van entre 0 y unas pocas $\mathrm{Kcal} / \mathrm{mol}$. Es común expresar el valor en unidades de $\mathrm{eV}$.

La energía de reorganización de esfera externa, puede ser estimada con la ecuación:

$$
\begin{aligned}
& \lambda_{\text {esfera extema }}=e^{2}\left(\frac{1}{2 r_{D}}+\frac{1}{2 r_{A}}-\frac{1}{r_{A D}}\right)\left(\frac{1}{\varepsilon_{o p}}-\frac{1}{\varepsilon_{s}}\right) \\
& \mathrm{r}_{\mathrm{AD}}=\mathrm{r}_{\mathrm{A}}+\mathrm{r}_{\mathrm{D}}
\end{aligned}
$$

donde $\mathrm{r}_{\mathrm{A}} \mathrm{y}_{\mathrm{D}}$ son los radios atómicos de aceptor y donor, $\varepsilon_{o p}$ es la constante dieléctrica del medio que responde a la polarización (es el cuadrado del índice de refracción), y $\varepsilon_{s}$ es la constante dieléctrica estática o permitividad relativa, correspondiente al dipolo del solvente. Los valores de $\lambda_{\text {esfera externa }}$ son típicamentes del orden de $10 \mathrm{Kcal} / \mathrm{mol}$ (frecuentemente $\lambda_{\text {esfera externa }} \angle 40 \mathrm{Kcal} / \mathrm{mol}$ ). 
La expresión dada para $\lambda$ supone un modelo dieléctrico continuo para el solvente y un modelo de oscilador armónico para los términos vibracionales.

La predicción de una región invertida donde al aumentar el gradiente de potencial disminuye la reactividad ha sido un desafío para los experimentalistas.

Muchos resultados indican que $\mathrm{k}_{\mathrm{ET}}$ aumenta típicamente con el incremento del gradiente de potencial, como es de esperar intuitivamente, pero al hacerse aún más grande el gradiente de potencial el valor de $\mathrm{k}_{\mathrm{ET}}$ alcanza un valor límite, dado por el control difusional de la reacción. Es necesario en este tipo de comportamientos una expresión para $\Delta G^{\mp}$ que tienda asintóticamente a cero para valores muy negativos de $\Delta \mathrm{G}^{0}$, la relación de Rehm-Weller cumple este requerimiento y es:

$$
\Delta G^{\mp}=\frac{\Delta G^{0}}{2}+\left[\left(\frac{\Delta G^{0}}{2}\right)^{2}+\Delta G^{\mp}(0)^{2}\right]^{1 / 2}
$$

\section{v. Cálculo de la constante de velocidad para el proceso de transferencia de electrones}

En el cálculo clásico, cada estado estable y estado de transición que se supone que actúa en la reacción de transferencia de electrones es descripto utilizando la teoría electrostática clásica, y no se hace referencia explícita a la existencia de estados cuánticos para el medio de reacción o para las moléculas reaccionantes. Específicamente, los estados de los sistemas se definen mediante conceptos 
mecanísticos y sus energías por un modelo de cargas puntuales o esféricas inmersas en cavidades del medio de reacción. El medio se describe como un contínuo y la descripción de la energética del sistema se realiza sólo a través de su polarizabilidad y la constante dieléctrica. Ya que la velocidad de reacción depende de la población de los estados definidos mecanísticamente, se supone una distribución de Boltzmann sobre los mismos.

Las reacciones de transferencia de electrones que se considerarán son las llamadas de esfera externa, esto es, la interacción electrónica entre el donor y el aceptor es débil como en el caso de las reacciones entre dos centros metálicos que no comparten un ligando común. La etapa fundamental en un proceso bimolecular de transferencia de electrones es la transferencia de un electrón entre A y D en el complejo de encuentro [A*--D] en el mecanismo de la reacción de transferencia electrónica. Un problema muy importante en esta etapa es el hecho de que la configuración nuclear de equilibrio de las especies cambia cuando estas ganan o pierden un electrón. En soluciones fluidas, estos cambios de configuración pueden involucrar cambios en distancias de enlace y ángulos y en la repolarización del solvente ${ }^{3 \mathrm{a}, 3 \mathrm{~b}, 52-55}$. Dado que los movimientos electrónicos son mucho más rápidos que los movimientos nucleares (Principio de Franck-Condon), en el tratamiento clásico se requiere un ajuste de las configuraciones nucleares previo a la transferencia de electrones. Esto lleva a la aparición de una barrera de activación, $\Delta G^{\mp}$, como se muestra en la figura 3 . Cuando se ha alcanzado una configuración nuclear apropiada, que ocurra o no la transferencia de electrones es un problema que depende de factores electrónicos. 
En una aproximación clásica, utilizando la teoría de las velocidades absolutas de reacción, es posible obtener la constante de velocidad $k_{e}$ para la transferencia de electrones en términos de la energía de activación $\Delta G^{\mp}$ para la reacción ${ }^{10,56-59}$ :

$$
\mathrm{k}_{\mathrm{e}}=\kappa v_{\mathrm{N}} \exp \left(-\Delta G^{\mp} / \mathrm{RT}\right)
$$

donde $\kappa$ es la probabilidad de que ocurra la transferencia del electrón normalizada al número de veces que la molécula adquiere la configuración nuclear correcta para pasar a través de la intersección de las curvas de energía potencial de los reactivos y los productos y $v_{\mathrm{N}}$ es una frecuencia nuclear efectiva para el movimiento nuclear.

La expresión para la energía de activación, $\Delta G^{\mp}$ en función de la energía libre de reacción, $\Delta G^{0}$ según la teoría de Marcus está dada por

$$
\Delta \mathrm{G}^{\mp}=\Delta \mathrm{G}^{\mp}(0)\left[1+\frac{\Delta \mathrm{G}^{0}}{4 \Delta \mathrm{G}^{\mp}(0)}\right]^{2}
$$

La expresión para la energía de activación, $\Delta G^{\mp}$ en función de la energía libre de reacción, $\Delta G^{0}$ según la teoría de Rehm-Weller está dada por:

$$
\Delta G^{\mp}=\frac{\Delta G^{0}}{2}+\left[\left(\frac{\Delta G^{0}}{2}\right)^{2}+\Delta G^{\mp}(0)^{2}\right]^{1 / 2}
$$


donde $\Delta G^{\mp}(0)$ es la llamada barrera nuclear intrínseca y representa la energía de activación cuando el cambio de energía libre de la reacción de transferencia de electrones es nulo (ver figura 3). Utilizando la expresión de la ecuación 10 para la constante de desactivación observada, $\mathrm{k}_{\mathrm{q}}$, $\mathrm{y}$ las ecuaciones 26,27 y 28 se obtiene:

$$
\mathrm{k}_{\mathrm{q}}=\frac{\mathrm{k}_{\mathrm{d}}}{1+\frac{\mathrm{k}_{\mathrm{d}}}{\kappa v_{\mathrm{N}} \mathrm{K}_{\mathrm{d}}} \exp \left[\frac{\Delta \mathrm{G}^{\mp}(0)}{\mathrm{RT}}\left(1+\frac{\Delta \mathrm{G}^{0}}{4 \Delta \mathrm{G}^{\mp}(0)}\right)^{2}\right]}
$$

$$
\mathrm{k}_{\mathrm{q}}=\frac{\mathrm{k}_{\mathrm{d}}}{1+\frac{\mathrm{k}_{\mathrm{d}}}{\kappa \nu_{\mathrm{N}} \mathrm{K}_{\mathrm{d}}} \exp \left[\frac{\Delta G^{0} / 2+\left[\left(\Delta G^{0} / 2\right)^{2}+\Delta G^{\mp}(0)^{2}\right]^{1 / 2}}{R T}\right]}
$$

donde $K_{d}$ es la constante de equilibrio para el complejo de encuentro $\left(K_{d}=k_{d} / k_{-d}\right)$.

Las ecuaciones 29 y 30 expresan la constante kq en función de la energía de activación según la teoría de Marcus y Rehm-Weller respectivamente. Estas ecuaciones fueron la utilizadas para ajustar los datos experimentales de $\log \mathrm{kq}$ versus $\Delta \mathrm{G}^{0}$ obtenidos de la desactivación no radiativa del polímero y del monómero con aminas.

De acuerdo con Smoluchowski ${ }^{60}$, la constante de velocidad $k_{d}$ puede escribirse como: 


$$
\mathrm{k}_{\mathrm{d}}=\frac{2 \mathrm{RT}}{3000 \eta}\left(\frac{1}{\mathrm{r}_{\mathrm{D}}}+\frac{1}{\mathrm{r}_{\mathrm{A}}}\right)\left(\mathrm{r}_{\mathrm{D}}+\mathrm{r}_{\mathrm{A}}\right)
$$

La constante de equilibrio para el complejo de encuentro $\left(\mathrm{K}_{\mathrm{d}}\right)$, siguiendo a Fuoss y a Eigen $^{61}$ puede obtenerse de:

$$
\mathrm{K}_{\mathrm{d}}=\frac{\mathrm{k}_{\mathrm{d}}}{\mathrm{k}_{-\mathrm{d}}}=\frac{4 \pi \mathrm{R}^{3} \mathrm{~N}_{\mathrm{A}}}{3000}
$$

donde $\mathrm{N}_{\mathrm{A}}$ es el número de Avogadro.

Para una serie homogénea de reacciones de transferencia de electrones tales como aquellas entre el mismo oxidante $A^{*}$ y una serie de donores $D_{1}, D_{2}, D_{3}$, etc, estructuralmente relacionados que tienen potenciales redox variables pero similar tamaño y forma e igual carga electrónica, se puede suponer que a través de la serie los parámetros $\mathrm{k}_{\mathrm{d}}, \mathrm{k}_{-\mathrm{d}}, \kappa, v_{\mathrm{N}}$ y $\lambda$ son constantes. Bajo esta suposición, $\mathrm{k}_{\mathrm{q}}$ en las ecuaciones 29 y 30 es sólo función de $\Delta \mathrm{G}^{0}$, y una gráfica de $\log \left(\mathrm{k}_{\mathrm{q}}\right)$ vs. $\Delta \mathrm{G}$ es una curva en forma de campana, según la teoría de Marcus, ecuación 29, que presenta: (i) una región “normal" para las reacciones endoergónicas y levemente exoergónicas, donde $\log \left(\mathrm{k}_{\mathfrak{q}}\right)$ se incrementa cuando aumenta la fuerza impulsora y llega a un máximo cuando $\Delta \mathrm{G}=-\lambda$, (ii) una región "invertida", para las reacciones fuertemente exoergónicas en la cual $\log \left(\mathrm{k}_{\mathrm{q}}\right)$ disminuye cuando aumenta la fuerza impulsora.

La observación de la región invertida para reacciones bimoleculares es a menudo muy difícil debido a que el máximo de la curva en forma de campana es cortado por la 
difusión. Para estos casos los datos pueden ser ajustados con la ecuación 30, que es la expresión de kq en función de $\Delta \mathrm{G}^{0}$ que surge de la teoría de Rehm-Weller y tiende asintóticamente a cero para reacciones muy exoergónicas. 


\section{Resultados experimentales}

\section{i. Desactivación no radiativa de la luminiscencia del polímero \\ $\left\{(v p y)_{2} v p y \operatorname{Re}(\mathrm{CO})_{3}\left(2,2^{\prime} \mathrm{Bpy}\right)^{+}\right\}_{n \sim 200}$ por aminas}

Se estudió la desactivación no radiativa del estado excitado emisor del complejo de Renio $-\operatorname{Re}(\mathrm{CO})_{3}\left(2,2^{\prime} \mathrm{Bpy}\right)^{+}$presente en el polímero $\left\{(\mathrm{vpy})_{2} \mathrm{vpyRe}(\mathrm{CO})_{3}\left(2,2^{\prime} \mathrm{Bpy}\right)^{+}\right\}_{200}$, figura 6 , sobre soluciones preparadas en acetonitrilo utilizando como desactivadores diferentes aminas alifáticas y aromáticas.

El polímero fue sintetizado y purificado siguiendo los pasos de literatura ${ }^{62}$.

Para las medidas de desactivación de la luminiscencia del estado excitado emisor (TCML) se utilizaron dos equipos diferentes. Algunas medidas se realizaron en un espectrofluorómetro, descripto en las técnicas experimentales y otras se realizaron determinando la disminución del tiempo de vida del estado excitado utilizando la técnica de láser flash fotólisis con un láser de $\mathrm{N}_{2} \lambda_{\text {exc }}: 337 \mathrm{~nm}$ y un láser Nd-Yag $\lambda_{\text {exc }}: 355 \mathrm{~nm}$.

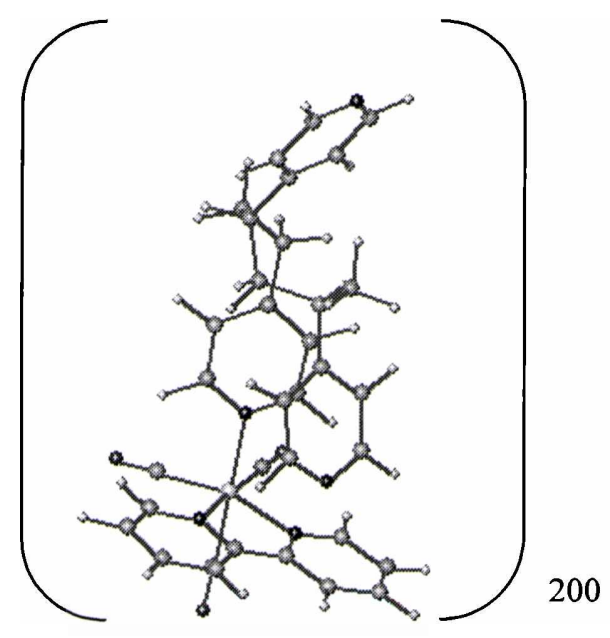

Figura 6: Representación esquemática de $\left\{(\mathrm{vpy})_{2} \mathrm{vpyRe}(\mathrm{CO})_{3}\left(2,2^{\prime} \mathrm{Bpy}\right)^{+}\right\}_{200}$ 
La concentración del complejo de $\operatorname{Re}^{\mathrm{I}}$ en todos los experimentos fue $5 \times 10^{-5} \mathrm{M}$ y la concentración de las aminas se varió entre $1 \times 10^{-5}$ y $1 \times 10^{-2} \mathrm{M}$. Las aminas utilizadas en los experimentos de desactivación no radiativa fueron: (1) $N, N_{,} N^{\prime}, N^{\prime}$ tetrametil 1,4 fenilendiamina, (2) 1,4 fenilendiamina, (3) 1,2 fenilendiamina, (4) 1,4 diazabiciclo [2.2.2] octano (DABCO), (5) N,N,N',N' tetrametil etilendiamina, (6) $\mathrm{N}, \mathrm{N}$ diisopropil etil amina, (7) Trietanolamina, (8) Trietilamina, (9) N,N,N',N' tetrametil diamino metano, (10) Dietilamina, (11) Dibencilamina y (12) n-Butilamina, ver esquema.

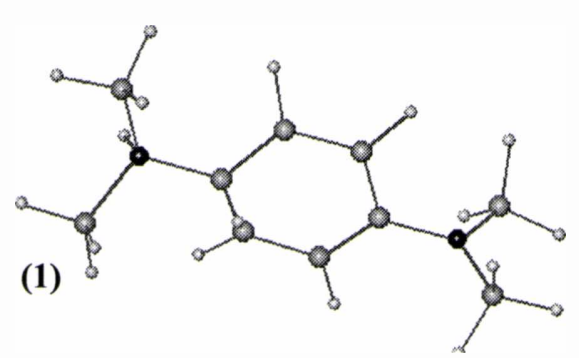

(3)

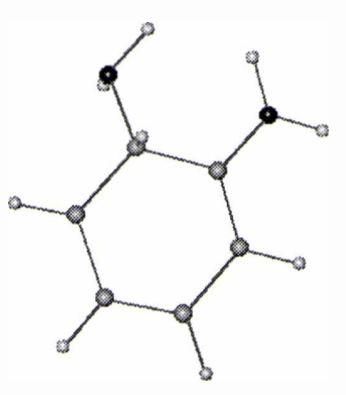

(2)

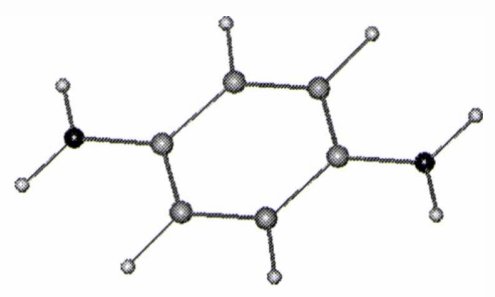

(4)

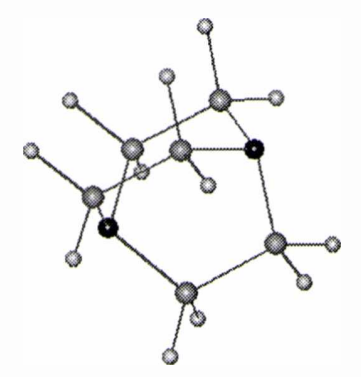




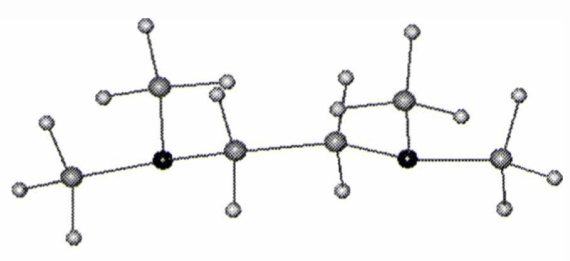

(5)

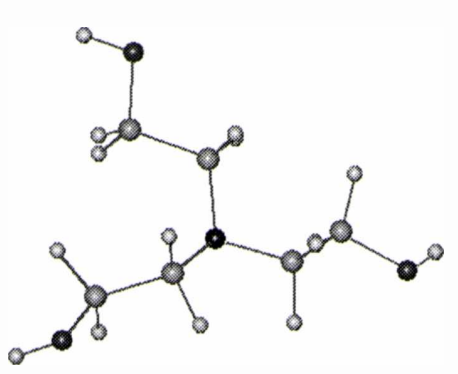

(7)<smiles>C[13C](=O)OC(=O)OC(=O)OC(=O)OC(=O)O</smiles>

(9)

(6)

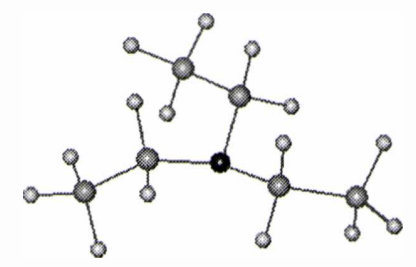

(8)

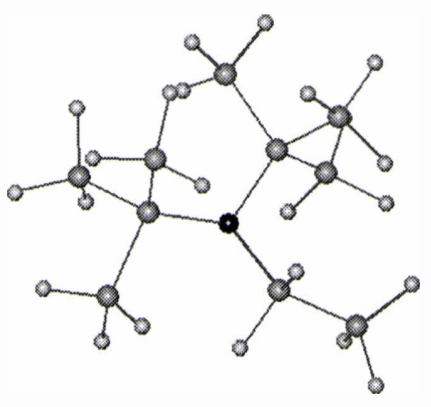

(10) 


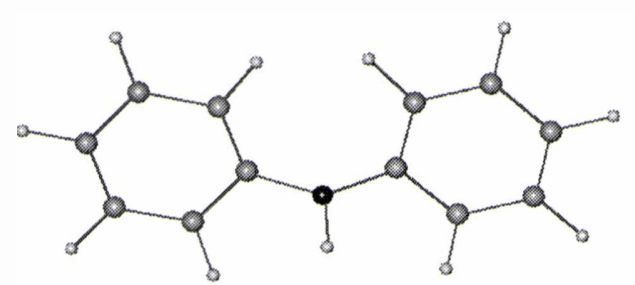

(11)

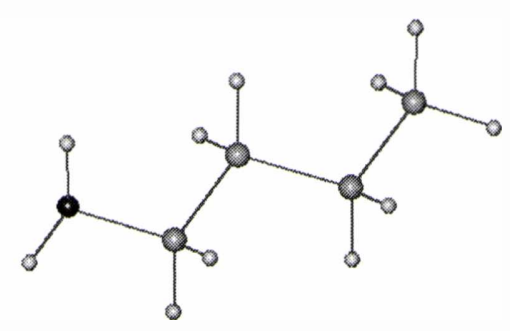

(12)

Representación esquemática de las aminas utilizadas, donde los átomos de Carbono están representados por ${ }^{\bullet}$, los átomos de Nitrógeno están representados por $\bullet$, los átomos de Oxígeno están representados por $\bullet$ y los átomos de Hidrógeno están representados por $\bigcirc$

En todos los casos estudiados, la desactivación de la emisión siguió una cinética típica de un mecanismo de Stern-Volmer:

$$
\phi_{0} / \phi=1+\mathrm{Ksv}[\text { amina }]
$$

ó

$$
\tau_{0} / \tau=1+K_{s v}[\text { amina }]
$$

donde $\phi_{0}$ representa el rendimiento cuántico de emisión de $\left\{(\mathrm{vpy})_{2} \mathrm{vpyRe}(\mathrm{CO})_{3}\left(2,2^{\prime} \mathrm{Bpy}\right)^{+}\right\}_{200}$ en ausencia de desactivador ([amina] $=0$ ), $\phi$ representa el rendimiento cuántico de emisión del complejo cuando la concentración de 
la amina es [amina] Esta expresión se utilizó en el caso en que los experimentos de desactivación se realizaron por determinación de la disminución del rendimiento cuántico de emisión.

$\tau_{0}$ es el tiempo de vida de emisión del polímero en ausencia de amina y $\tau$ es el tiempo de vida del complejo cuando la concentración de amina es [amina], esta expresión fue utilizada cuando los experimentos de desactivación se realizaron con la técnica de láser flash fotólisis utilizando un láser de $\mathrm{N}_{2}$ de $\lambda: 337 \mathrm{~nm}$ ó un láser Nd-Yag de $\lambda: 355 \mathrm{~nm}$.

y Ksv es la constante de Stern-Volmer.

Teniendo en cuenta el tiempo de vida de emisión del estado excitado $\left(\tau_{0}\right)$ en ausencia de quenching, se puede calcular la constante de desactivación bimolecular $\left(\mathrm{k}_{\mathrm{q}}\right)$ como:

$$
\mathrm{k}_{\mathrm{q}}=\mathrm{K}_{\mathrm{sv}} / \tau_{0}
$$

Los resultados de la desactivación no radiativa de la emisión de $\left\{(\mathrm{vpy})_{2} \mathrm{vpyRe}(\mathrm{CO})_{3}\left(2,2^{\prime} \mathrm{Bpy}\right)^{+}\right\}_{\mathrm{n} \sim 200}$ por las aminas representados como gráficos de Stern-Volmer se muestran en las figuras 7 a 9. 

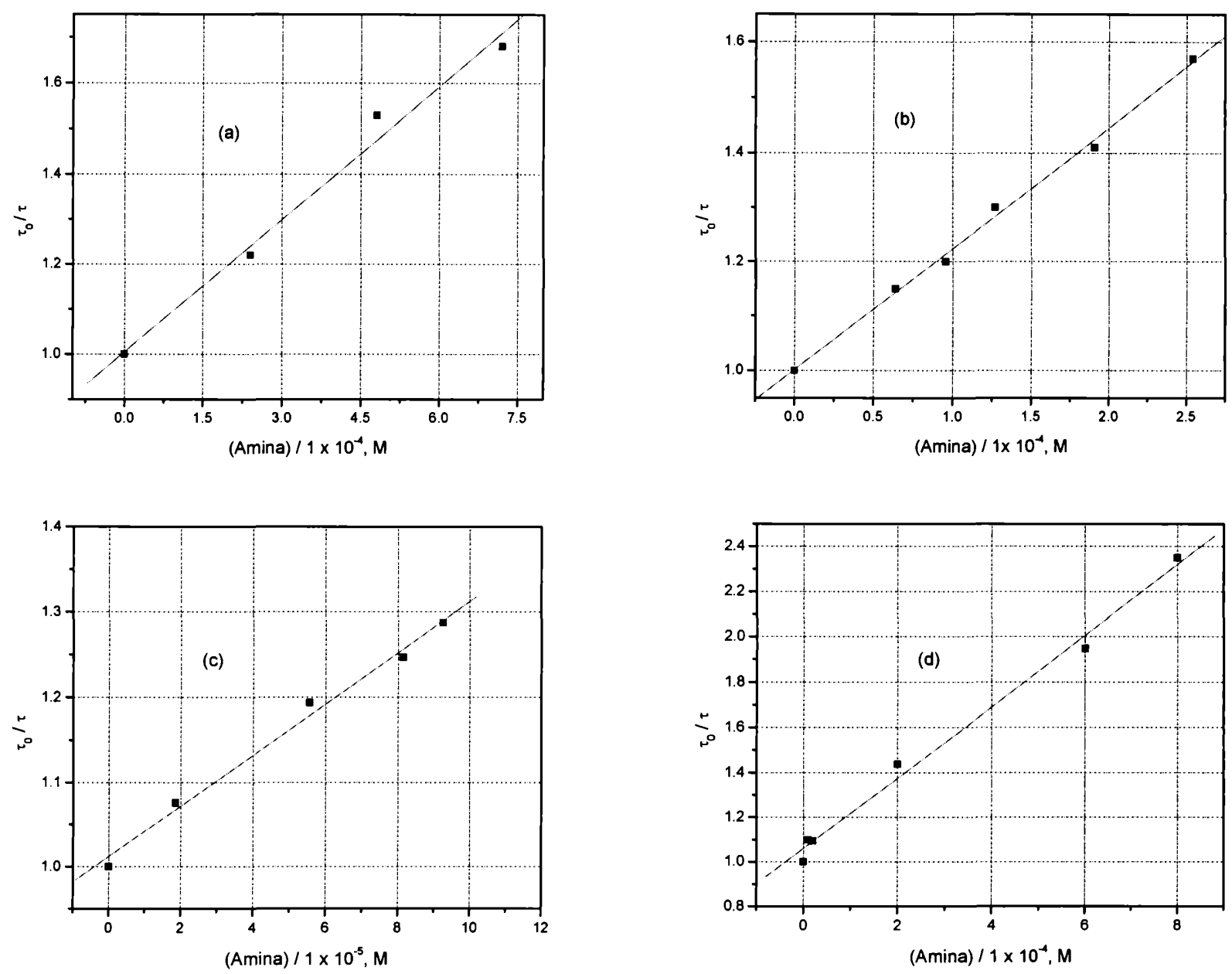

Figura 7: Cinética de Stern-Volmer para la desactivación de la emisión de $\left\{(\mathrm{vpy})_{2} \mathrm{vpy} \operatorname{Re}(\mathrm{CO})_{3}(\mathrm{Bpy})^{+}\right\}_{200}$ con aminas: (a) N,N,N',N'tetrametil 1,4-fenilendiamina; (b) 1,4-fenilendiamina; (c) 1,2-fenilendiamina y (d) Dabco. 

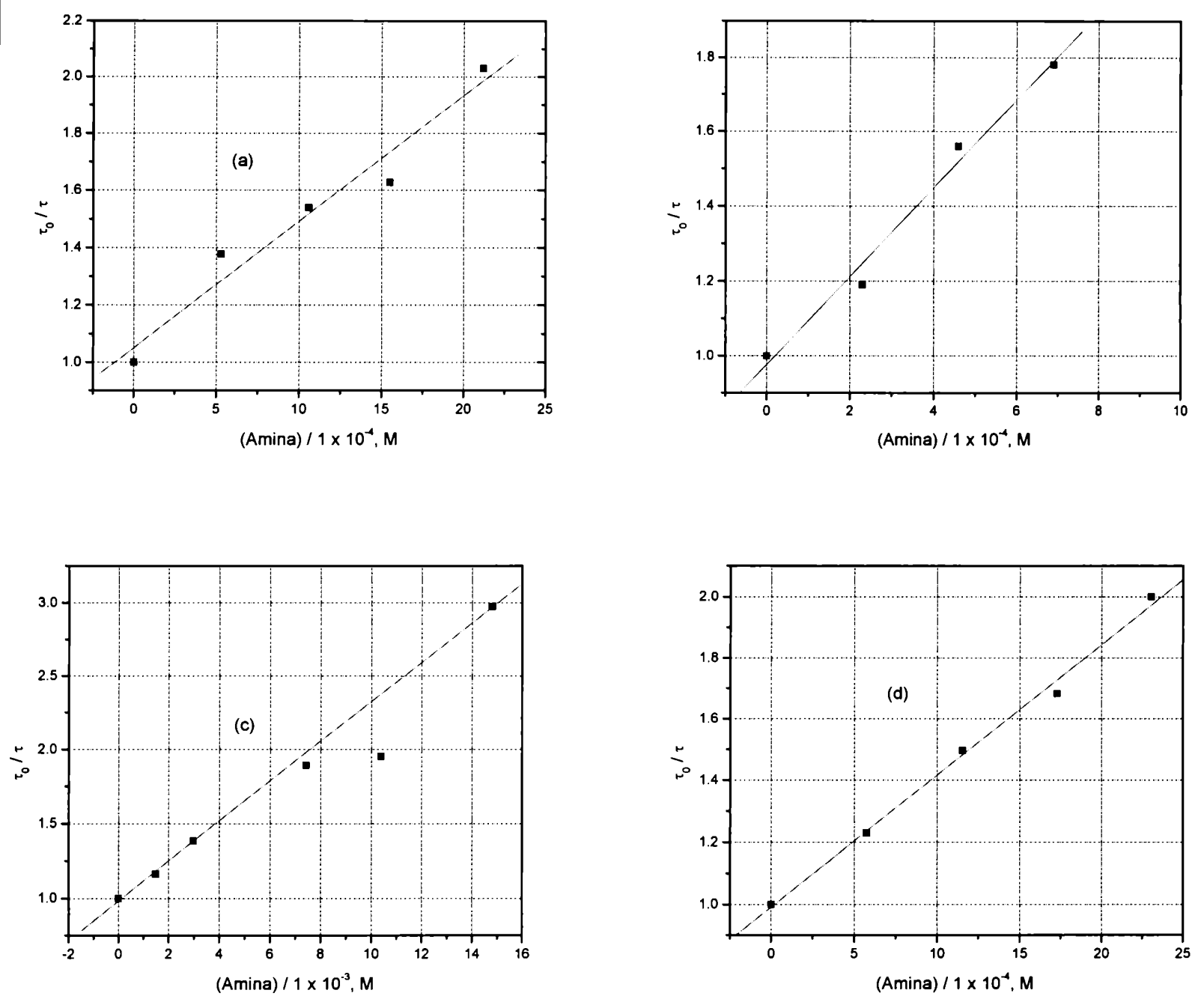

Figura 8: Cinética de Stern-Volmer para la desactivación de la emisión de $\left\{(\mathrm{vpy})_{2} \mathrm{vpyRe}(\mathrm{CO})_{3}(\mathrm{Bpy})^{+}\right\}_{200}$

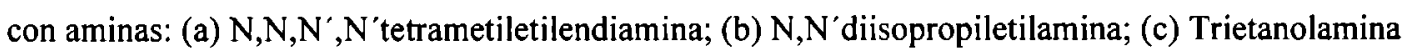
y (d) trietilamina. 

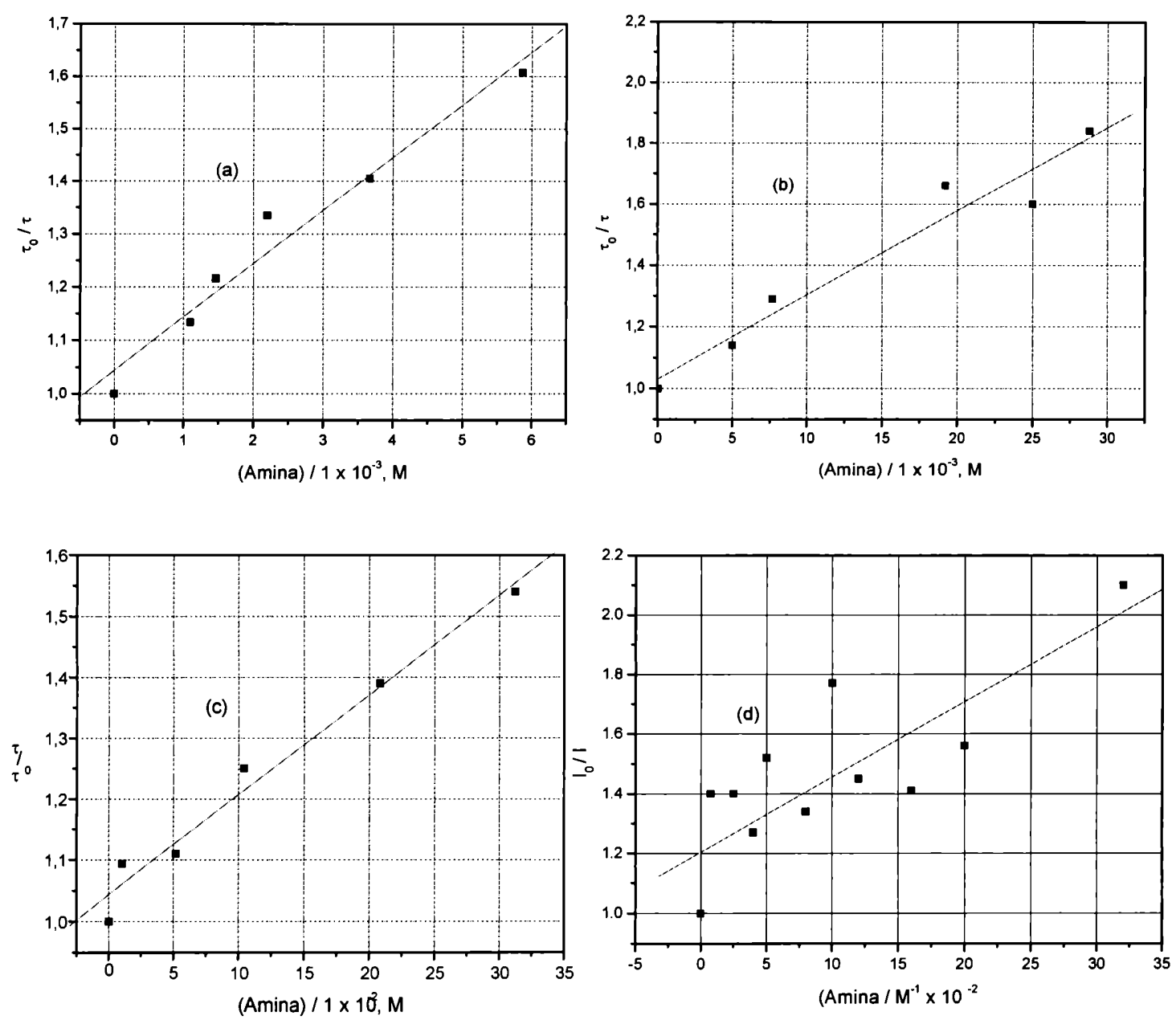

Figura 9: Cinética de Stern-Volmer pla desactivación de la emisión de $\left\{(\mathrm{vpy})_{2} \mathrm{vpy} \operatorname{Re}(\mathrm{CO})_{3}(\mathrm{Bpy})^{+}\right\}_{200}$ con aminas: (a) N,N,N',N'tetrametildiaminometano; (b) Dietilamina; (c) Dibencilamina y (d) n-butilamina. 


\section{ii. Desactivación no radiativa de la luminiscencia del monómero $\mathrm{Py} \operatorname{Re}(\mathrm{CO})_{3}\left(2,2^{\prime} \mathrm{Bpy}\right)^{+}$}

\section{por aminas}

La desactivación no radiativa del monómero $\operatorname{PyRe}(\mathrm{CO})_{3}\left(2,2^{\prime} \mathrm{Bpy}\right)^{+}$,figura 10 , que fue sintetizado y purificado según literatura ${ }^{62}$ se estudió con los mismos métodos y con las mismas aminas utilizadas para el estudio de la desactivación no radiativa del polímero $\left\{(\mathrm{vpy})_{2} \mathrm{vpyRe}(\mathrm{CO})_{3}\left(2,2^{\prime} \mathrm{Bpy}\right)^{+}\right\}_{200}$. Los resultados se muestran en las figuras 11 a 13.

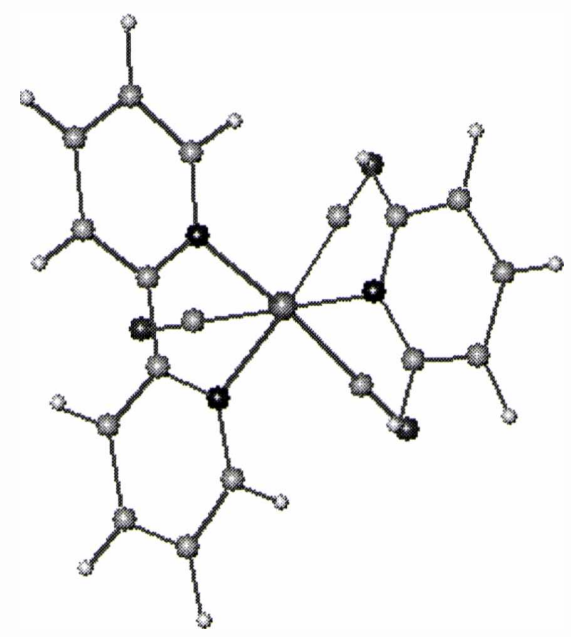

Figura 10: Representación esquemática de $\left.\operatorname{PyRe}(\mathrm{CO})_{3}\left(2,2^{\prime} \mathrm{Bpy}\right)^{+}\right\}$ 


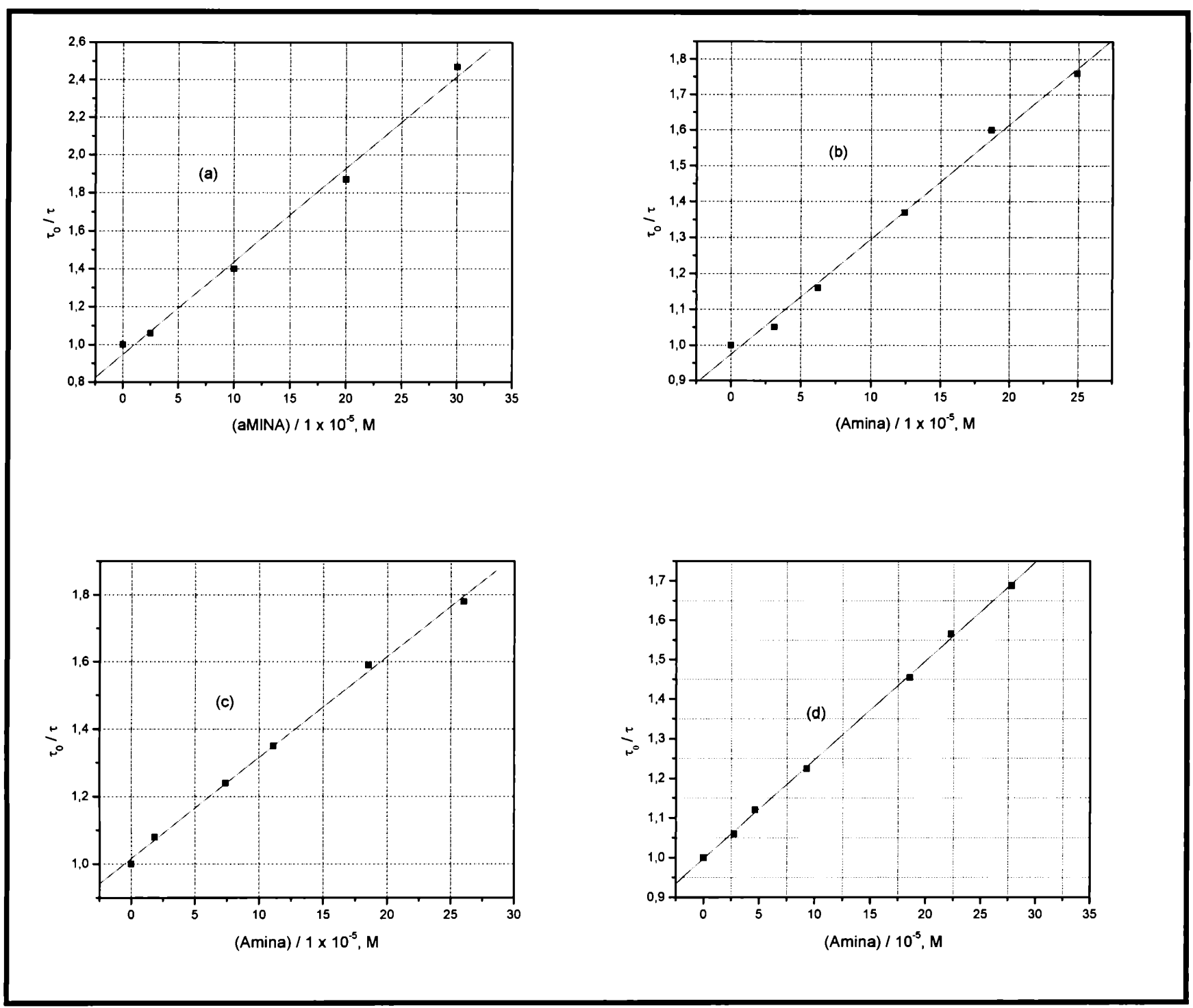

Figura 11: Cinética de Stern-Volmer para la desactivación de la emisión de $\operatorname{PyRe}(\mathrm{CO})_{3}\left(2,2^{\prime} \mathrm{Bpy}\right)^{+}$con aminas: (a) $\mathrm{N}, \mathrm{N}, \mathrm{N}^{\prime}, \mathrm{N}^{\prime}$ tetrametil 1,4-fenilendiamina; (b) 1,4-fenilendiamina; (c) 1,2-fenilendiamina y (d) Dabco. 


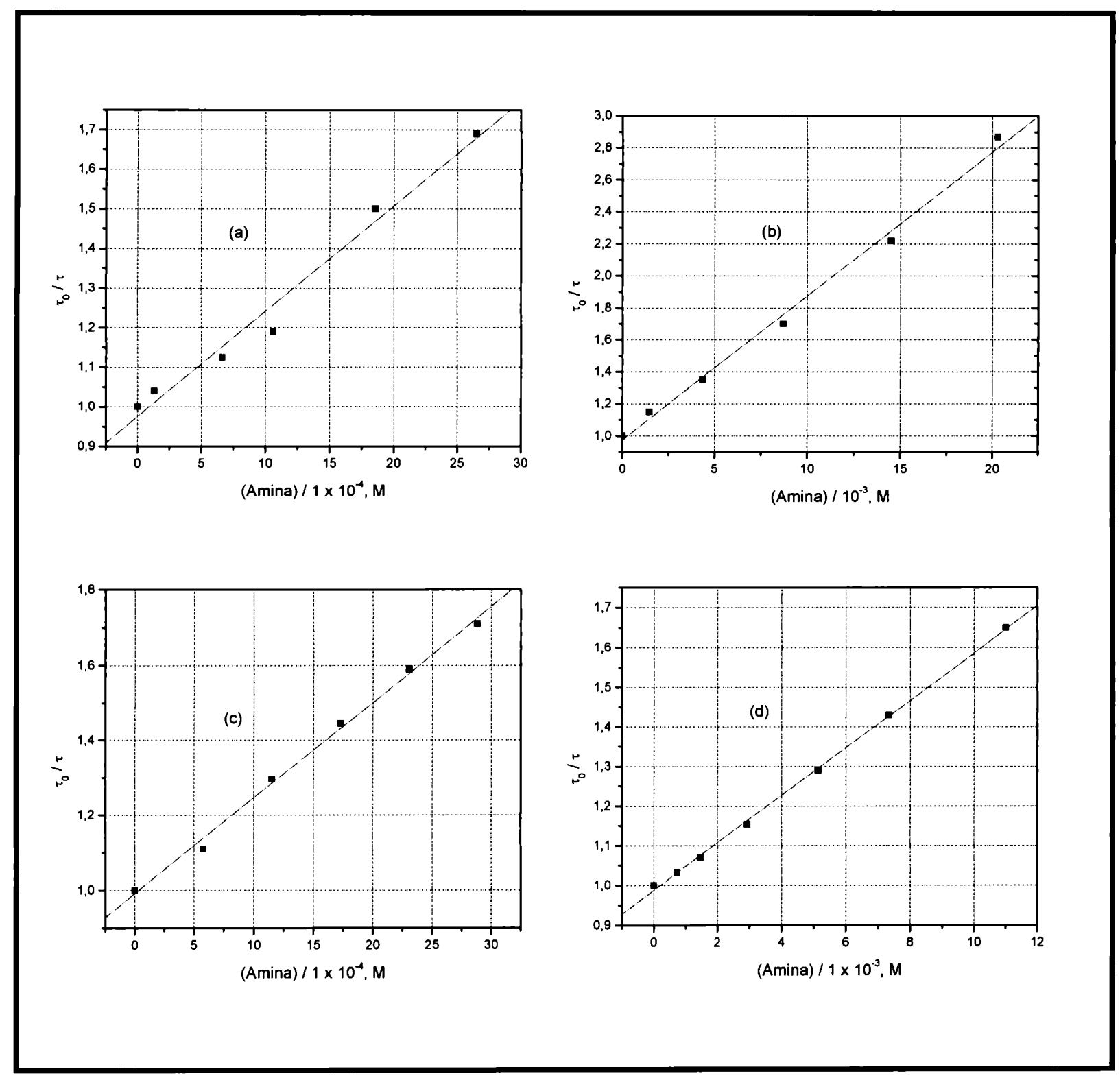

Figura 12: Cinética de Stern-Volmer para la desactivación de la emisión de $\left\{(\mathrm{vpy})_{2} \mathrm{vpyRe}(\mathrm{CO})_{3}(\mathrm{Bpy})^{+}\right\}_{\mathrm{n}-200}$ con aminas: (a) $\mathrm{N}, \mathrm{N}, \mathrm{N}^{\prime}, \mathrm{N}^{\prime}$ tetrametiletilendiamina; (b) Trietanolamina, (c) trietilamina y (d) $\mathrm{N}, \mathrm{N}, \mathrm{N}^{\prime}, \mathrm{N}^{\prime}$ tetrametildiaminometano 


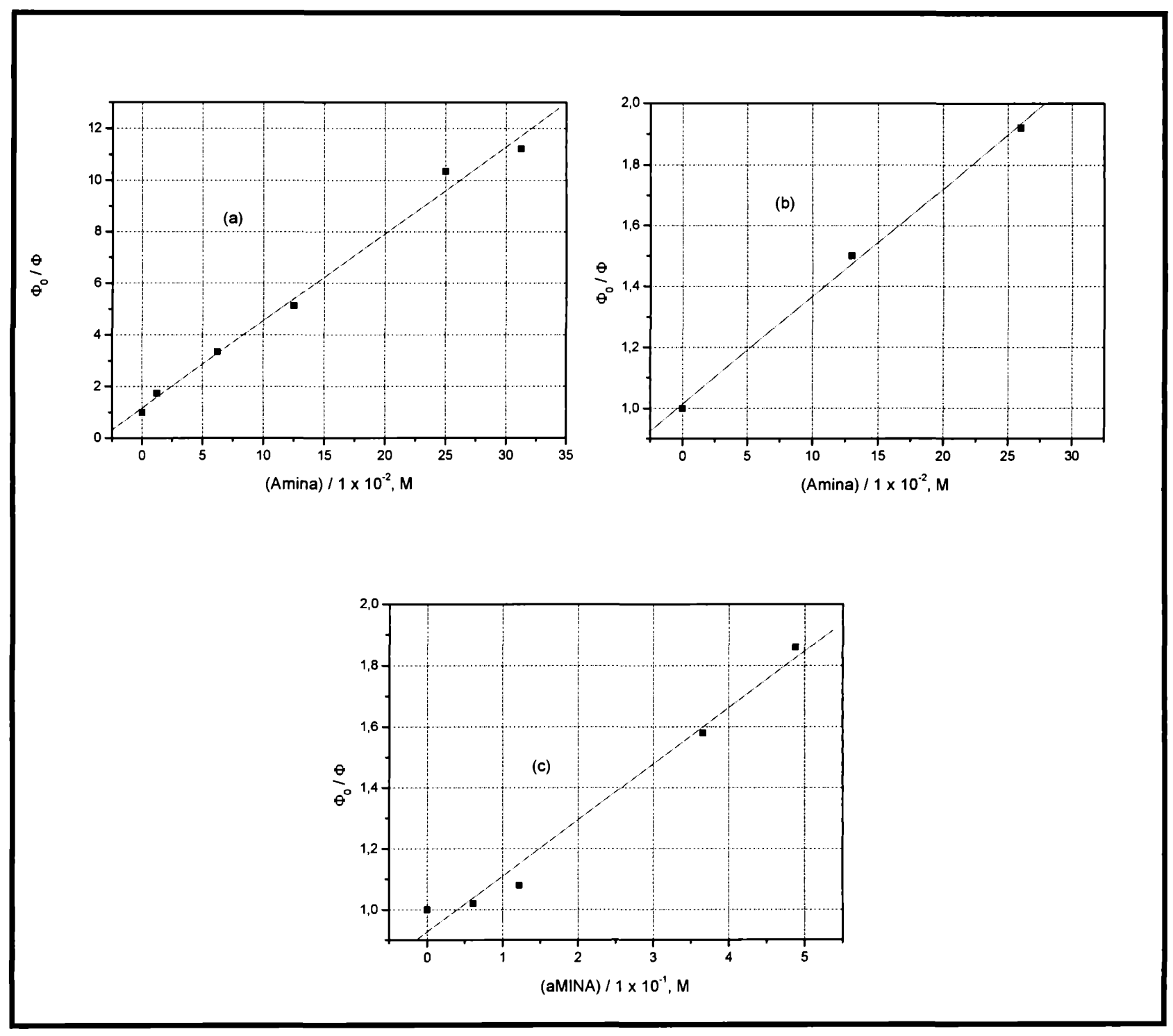

Figura 13: Cinética de Stern-Volmer para la desactivación de la emisión de $\operatorname{PyRe}(\mathrm{CO})_{3}(\mathrm{Bpy})^{+}$con aminas:

(a) Dietilamina; (b) Dibencilamina y (c) n-butilamina. 
En la tabla 1 se muestran los valores de kq obtenidos para el monómero y el polímero con las diferenctes aminas. Además se incluye el valor de $\Delta G^{0}$ calculado con la ecuación 17 :

$$
\Delta G^{0} \approx E_{D^{+} / D}^{0}-E_{A / A^{-}}^{0}-E_{D^{*}}
$$

Donde $\mathrm{E}_{\mathrm{D}^{*}}$ fue estimado del espectro de emisión del monómero $\operatorname{PyRe}(\mathrm{CO})_{3}(\mathrm{Bpy})^{+}$, dando un valor de $\mathrm{E}_{\mathrm{D}^{*}}=2.37 \mathrm{eV}$.

$E_{D^{+} / D}^{0} y E_{A / A^{-}}^{0}$, son los potenciales de reducción del cromóforo $-\operatorname{Re}(\mathrm{CO})_{3}\left(2,2^{\prime} \mathrm{Bpy}\right)^{+63} \mathrm{y}$ de la amina. 


\begin{tabular}{|c|c|c|c|c|}
\hline & & & Polímero & Monómero \\
\hline Desactivador & $\mathrm{E}_{\mathrm{ox}} / \mathrm{eV}$ & $\Delta \mathrm{G}^{0} / \mathrm{eV}$ & $\mathrm{Kq} / \mathrm{M}^{-1} \mathrm{~s}^{-1}$ & $\mathrm{Kq} / \mathrm{M}^{-1} \mathrm{~s}^{-1}$ \\
\hline $\mathrm{N}, \mathrm{N}, \mathrm{N}^{\prime} \mathrm{N}^{\prime}$ tetrametil 1,4-fenilendiamina & 0.13 & $-1,15$ & $4,8 \times 10^{9}$ & $2 \times 10^{10}$ \\
\hline 1,4 -fenilendiamina & 0.20 & $-1,08$ & $1,1 \times 10^{10}$ & $1,31 \times 10^{10}$ \\
\hline 1,2-fenilendiamina & 0.40 & $-0,88$ & $1,5 \times 10^{10}$ & $1,3 \times 10^{10}$ \\
\hline Dabco & 0.56 & $-0,72$ & $7,8 \times 10^{9}$ & $1,02 \times 10^{10}$ \\
\hline $\mathrm{N}, \mathrm{N}, \mathrm{N}^{\prime} \mathrm{N}^{\prime}$ tetrametiletilendiamina & 0.87 & $-0,41$ & $2,1 \times 10^{9}$ & $1,01 \times 10^{9}$ \\
\hline $\mathrm{N}, \mathrm{N}^{\prime}$ diisopropilamina & 0.89 & $-0,39$ & $5,8 \times 10^{9}$ & -- \\
\hline Trietanolamina & 0.90 & $-0,38$ & $6,1 \times 10^{8}$ & $3,2 \times 10^{8}$ \\
\hline Trietilamina & 0.99 & $-0,29$ & $2,1 \times 10^{9}$ & $1,04 \times 10^{9}$ \\
\hline $\mathrm{N}, \mathrm{N}, \mathrm{N}$ 'N'tetrametildiaminometano & 1.06 & $-0,22$ & $4,9 \times 10^{8}$ & $2,4 \times 10^{8}$ \\
\hline Dietilamina & 1.31 & 0,03 & $1,34 \times 10^{8}$ & $1,7 \times 10^{8}$ \\
\hline Dibencilamina & 1.36 & 0,08 & $8,1 \times 10^{6}$ & $8,36 \times 10^{6}$ \\
\hline n-butilamina & 1.39 & 0,11 & $1,5 \times 10^{7}$ & $3,84 \times 10^{6}$ \\
\hline
\end{tabular}

Tabla 1: Contantes de desactivación no radiativa del estado excitado de TCML del polímero $\left\{(\mathrm{vpy})_{2} \text { vpy } \operatorname{Re}(\mathrm{CO})_{3}(\mathrm{Bpy})^{+}\right\}_{\mathrm{n}-200}$ y el monómero PyRe $(\mathrm{CO})_{3}(\mathrm{Bpy})^{+} . \Delta \mathrm{G}^{0}$ para la reacción de transferencia de electrones entre el monómero y las aminas.

iii. Especies reducidas de $\left\{(v p y)_{2} v p y \operatorname{Re}(\mathrm{CO})_{3}\left(2,2^{\prime} \mathrm{Bpy}\right)^{+}\right\}_{n \sim 200}$ vía fotoquímica y por radiólisis de pulso

Para estudiar las especies reducidas del polímero $\left\{(\text { vpy })_{2} \text { vpyRe }(\mathrm{CO})_{3}\left(2,2^{\prime} \mathrm{Bpy}\right)^{+}\right\}_{200}$ generadas vía fotoquímica se realizaron experimentos de láser flash fotólisis con soluciones de $\left\{(\mathrm{vpy})_{2} \mathrm{vpyRe}(\mathrm{CO})_{3}\left(2,2^{\prime} \mathrm{Bpy}\right)^{+}\right\}_{200}$ preparadas 
en acetonitrilo, deaereadas con $\mathrm{N}_{2}$ ultrapuro y en presencia de trietilamina TEA en una concentración [TEA].

En el espectro registrado para el transiente reducido, $\Delta \mathrm{A}_{\max }$ en función de la longitud de onda, figura 14 , pueden observarse tres máximos a $\lambda=430,462$ y $490 \mathrm{~nm}$. Este espectro muestra los valores máximos del $\Delta \mathrm{A}$ registrados vs. la longitud de onda. Puede observarse de esta figura que $\Delta \mathrm{A}_{\max }$ es ligeramente mayor que $\Delta \mathrm{A}_{\mathrm{t}=0}$ (el $\Delta \mathrm{A}$ registrado inmediatamente después del disparo del pulso laser). Luego de este incremento en el $\Delta \mathrm{A}$ se observa el decaimiento de las trazas de absorción en la escala de los microsegundos, figura 15. Los decaimientos de las trazas de absorción en función del tiempo se ajustaron a una cinética de primer orden con un $\tau=4.9 \mu \mathrm{s}$. 


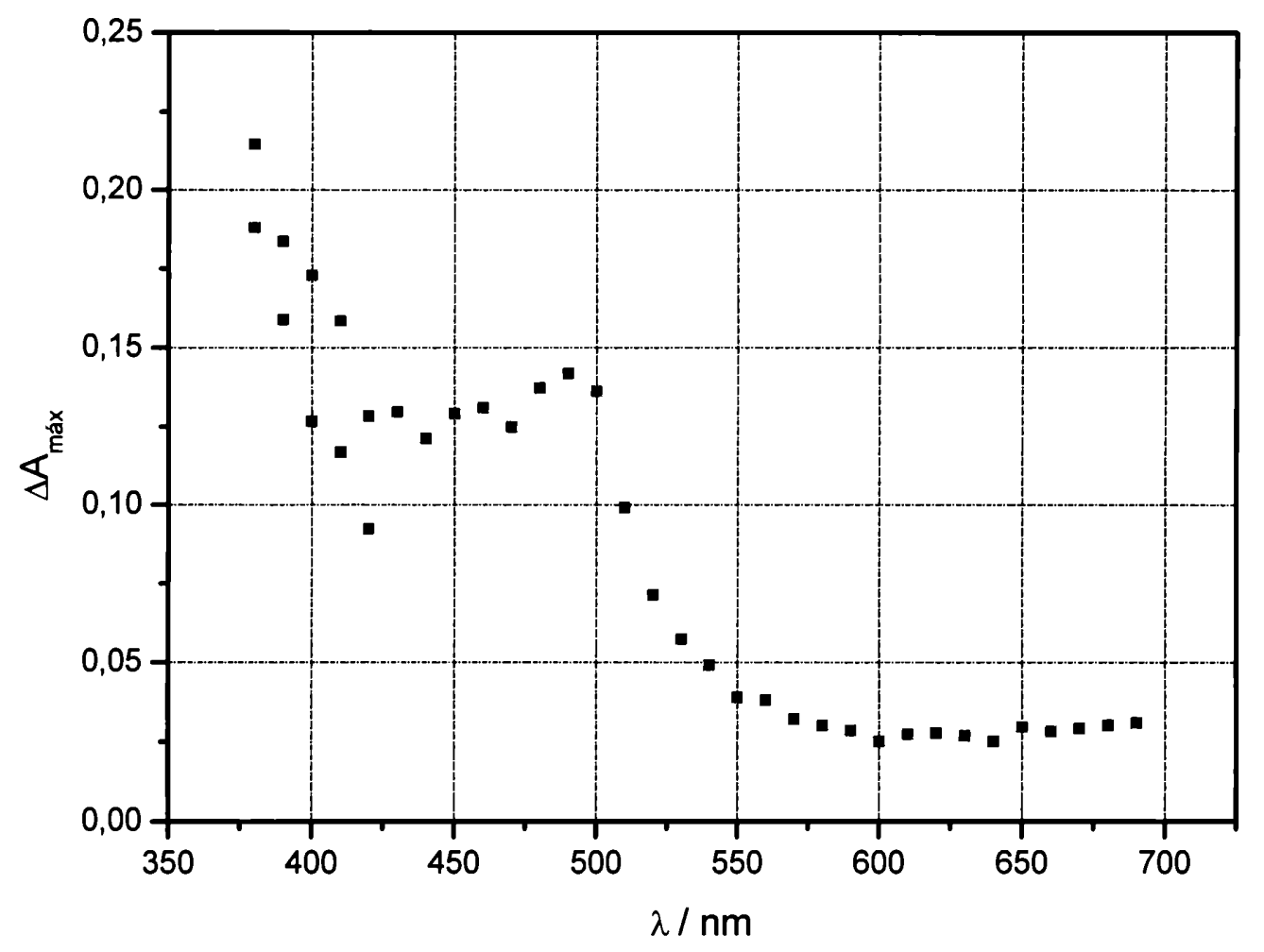

Figura 14: Espectro del transientes, registrado en experimentos de flash fotólisis del polímero, $\left\{\left[(\mathrm{vpy})_{2} \mathrm{vpyRe}^{\mathrm{I}}(\mathrm{CO})_{3}\left(2,2^{\prime} \mathrm{Bpy}\right)^{+}\right]\right\}_{200}\left(\left[\mathrm{Re}^{\mathrm{I}}\right]=1.6 \times 10^{-4} \mathrm{M}\right)$ en soluciones de $\mathrm{CH}_{3} \mathrm{CN}$ deaereadas con $\mathrm{N}_{2}$ ultrapuro en presencia de TEA en concentración $0.1 \mathrm{M}$. 

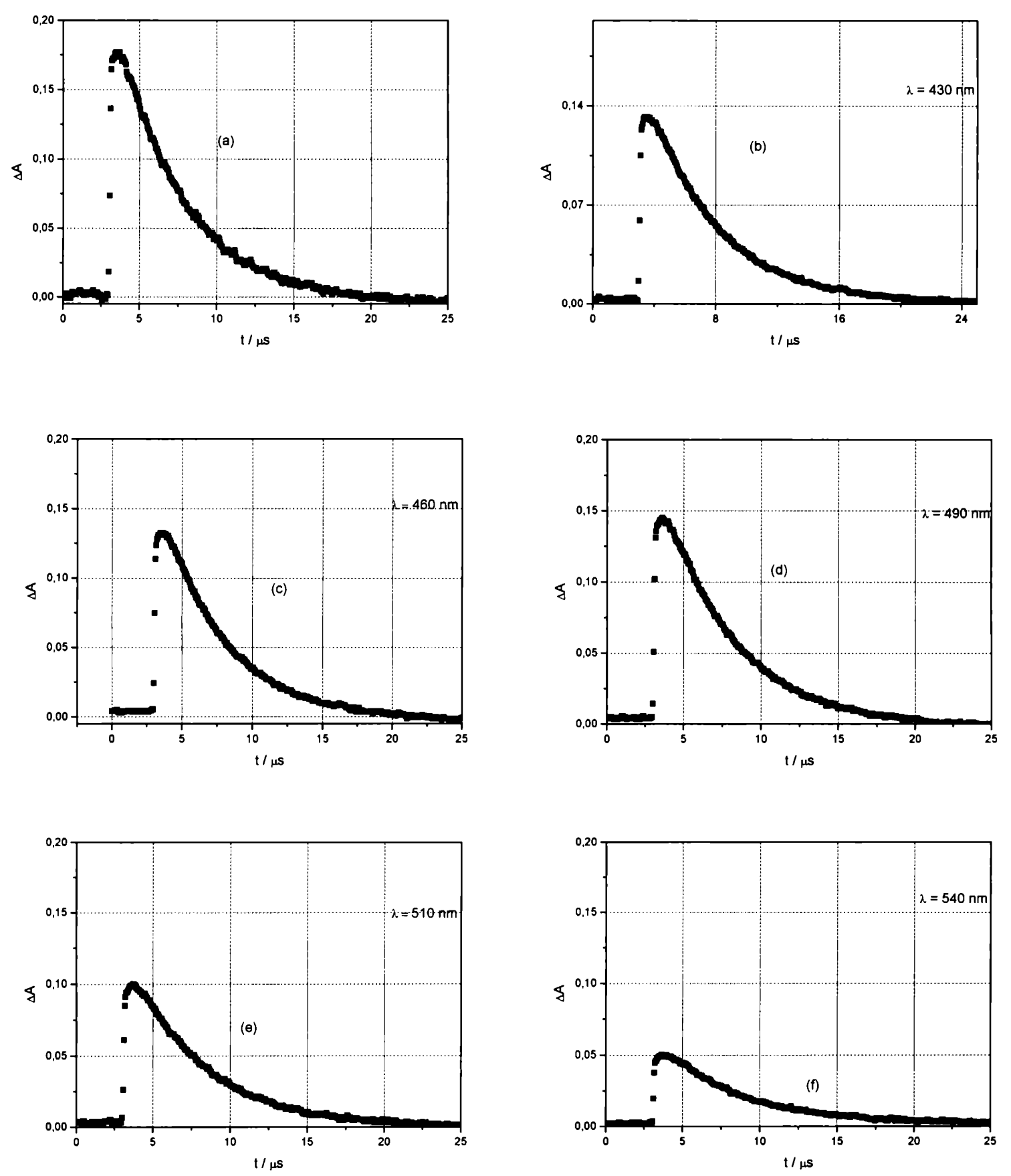

Figura 15: Cinética de decaimiento de absorbancia en función del tiempo del transiente reducido del polímero $\left\{(\text { vpy })_{2} \text { vpyRe }(\mathrm{CO})_{3}\left(2,2^{\prime} \mathrm{Bpy}\right)^{+}\right\}_{200}$ generado vía reacción fotoquímica con TEA en experimentos de láser flash fotólisis. (a) $\lambda_{\text {obs }}=400 \mathrm{~nm}$; (b) $\lambda_{\text {obs }}=430 \mathrm{~nm}$; (c) $\lambda_{\text {obs }}=460 \mathrm{~nm}$; (d) $\lambda_{\text {obs }}=490 \mathrm{~nm}$; (e) $\lambda_{\text {obs }}=510 \mathrm{~nm}$; (f) $\lambda_{\text {obs }}=540 \mathrm{~nm}$ 
Es posible atribuir el espectro de la figura 14 a la especie fotogenerada $-\operatorname{Re}^{\mathrm{l}}(\mathrm{CO})_{3}\left(2,2^{\prime} \mathrm{Bpy}\right)^{\circ}$. Esta asignación puede ser confirmada debido a que el mismo cromóforo fue generado con la técnica de radiólisis de pulso que se realizó al polímero $\left\{(\mathrm{vpy})_{2} \mathrm{vpyRe}(\mathrm{CO})_{3}\left(2,2^{\prime} \mathrm{Bpy}\right)^{+}\right\}_{n \sim 200}\left(\left[\mathrm{Re}^{\mathrm{l}}\right]=5.3 \times 10^{-5} \mathrm{M}\right)$ en $\mathrm{MeOH}$ deaereada con $\mathrm{N}_{2}$ ultrapuro. Como se describió en la Parte I, la radiólisis de soluciones preparadas en $\mathrm{CH}_{3} \mathrm{OH}$ y deaereadas con $\mathrm{N}_{2}$ ultrapuro, producen como principales productos $e_{\text {solv }}^{-}$y $\mathrm{CH}_{2} \mathrm{OH}^{\circ}$. Cuando las soluciones de polímero fueron deaereadas con $\mathrm{N}_{2} \mathrm{O}$ en lugar de $\mathrm{N}_{2}$, el pulso radiolítico no genera cambios en el espectro de la solución. Este experimento demuestra la falta de poder reductor del radical $\mathrm{CH}_{2} \mathrm{OH}^{\circ}$ sobre el ligando 2,2'Bpy, presente en el polímero de $\mathrm{Re}^{\mathrm{I}}$, y que los cambios que se reflejan en la figura 14 son generados por la reacción del polímero sólo con $e_{s o l v}^{-}$.

La reacción entre el $e_{s o l v}^{-}$, en experimentos de radiólisis de pulso y el polímero $\left\{(\text { vpy })_{2} \text { vpyRe }(\mathrm{CO})_{3}\left(2,2^{\prime} \mathrm{Bpy}\right)^{+}\right\}_{\mathrm{n} \sim 200}$ fue completa luego de los primeros microsegundos con una constante de velocidad de $(2.1 \pm 0.2) \times 10^{10} \mathrm{M}^{-1} \mathrm{~s}^{-1}$. El espectro generado por reacción del polímero con el $e_{\text {solv }}^{-}$se muestra en la figura 16 y este exhibe tres bandas de absorción con $\lambda_{\text {máx }}=420 \mathrm{~nm}, 460 \mathrm{~nm}$ y $490 \mathrm{~nm}$, este espectro coincide con el espectro fotogenerado con TEA. 


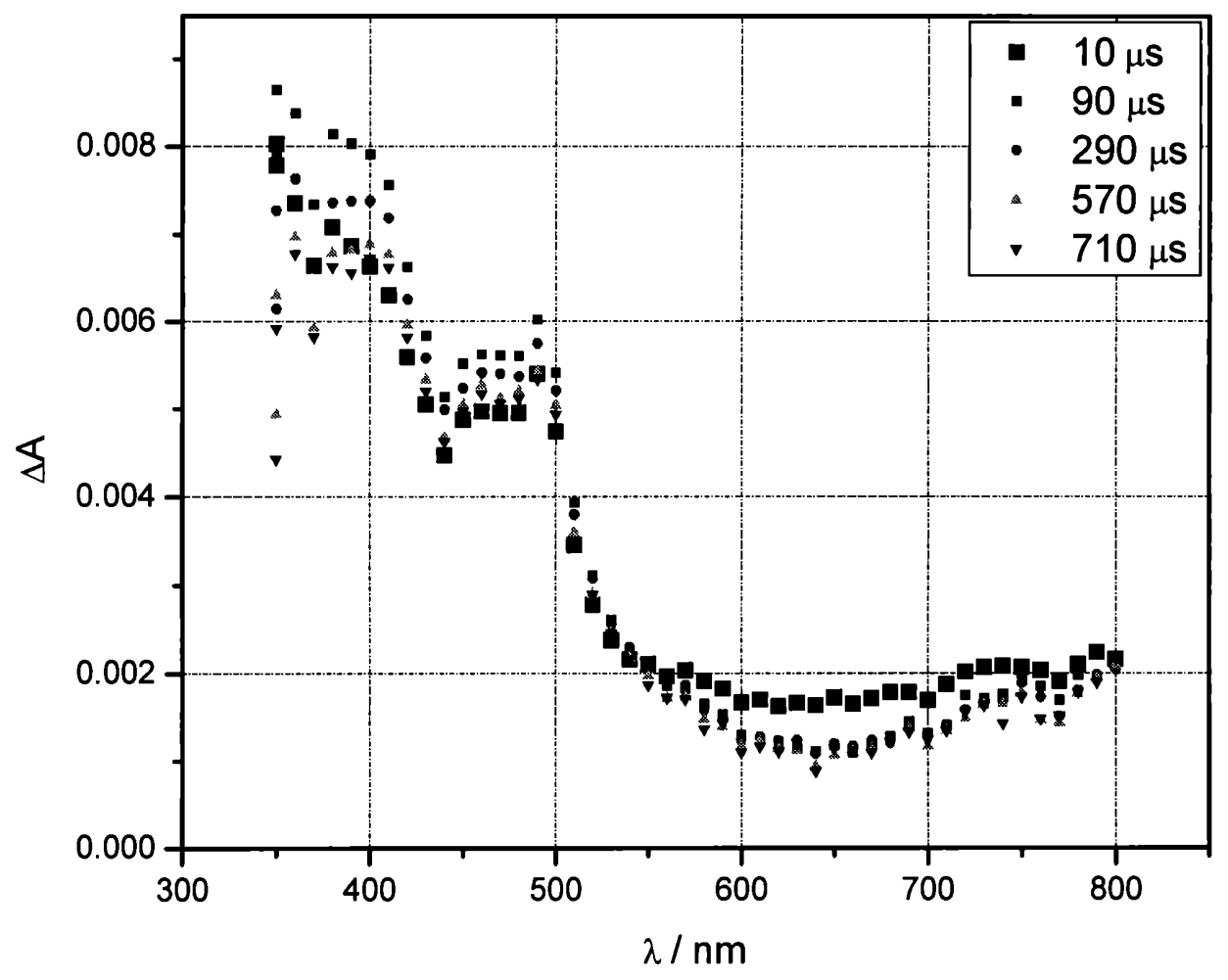

Figura 16 : Espectro del transientes registrado luego de un pulso radiolítico en experimentos de radiólisis de pulso sobre soluciones metanólicas deaereadas con $\mathrm{N}_{2}$ del polímero:

$\left\{\left[(\text { vpy })_{2} \operatorname{vpyRe}^{\prime}(\mathrm{CO})_{3}\left(2,2^{\prime} \mathrm{Bpy}\right)^{+}\right]\right\}_{\mathrm{n} \sim 200}$ 


\section{Cálculos y conclusiones}

De los experimentos de láser flash fotólisis sobre soluciones del polímero $\left\{(\mathrm{vpy})_{2} \mathrm{vpyRe}(\mathrm{CO})_{3}\left(2,2^{\prime} \mathrm{Bpy}\right)^{+}\right\}_{200}$ en presencia de TEA, puede observarse que el transiente reducido decae en la escala de los microsegundos, siguiendo una cinética de primer orden y con un tiempo de vida, $\tau=4.9 \mu \mathrm{s}$, figura 13. La reducción del estado excitado de TCML en el polímero por TEA, se produce en un tiempo menor a 25 ns. Además puede verse un crecimiento en $\Delta \mathrm{A}$, debido a la formación adicional de transientes reducido generado por reacción de $-\operatorname{Re}(\mathrm{CO})_{3}\left(2,2^{\prime} \mathrm{Bpy}\right)^{+}$con radicales reductores , como TEA', generados de la oxidación de la TEA:

$$
\left(\mathrm{CH}_{3} \mathrm{CH}_{2}\right)_{3} \mathrm{~N}^{\bullet+}+\mathrm{TEA} \rightarrow\left(\mathrm{CH}_{3} \mathrm{CH}_{2}\right)_{2} \mathrm{~N}\left(\mathrm{CH}_{2} \mathrm{CH}_{2}^{\bullet}\right)+\mathrm{TEAH}^{+} \text {(36) }
$$

De acuerdo a los resultados experimentales aquí presentados, puede plantearse un mecanismo general para la desactivación no radiativa de la emisión del cromóforo $-\operatorname{Re}(\mathrm{CO})_{3} \mathrm{Bpy}^{+}$, por aminas:

$$
\begin{aligned}
& -\operatorname{Re}(\mathrm{CO})_{3}\left(2,2^{\prime} \mathrm{Bpy}\right)^{+}+\mathrm{hu} \longrightarrow-\operatorname{Re}(\mathrm{CO})_{3}\left(2,2^{\prime} \mathrm{Bpy}\right)^{+^{*}} \\
& -\operatorname{Re}(\mathrm{CO})_{3}\left(2,2^{\prime} \mathrm{Bpy}\right)^{+^{*}} \longrightarrow-\operatorname{Re}(\mathrm{CO})_{3}\left(2,2^{\prime} \mathrm{Bpy}\right)^{+}+\text {calor } \\
& -\operatorname{Re}(\mathrm{CO})_{3}\left(2,2^{\prime} \mathrm{Bpy}\right)^{+^{*}} \longrightarrow-\operatorname{Re}(\mathrm{CO})_{3}\left(2,2^{\prime} \mathrm{Bpy}\right)^{+}+\mathrm{hu}^{\prime}
\end{aligned}
$$




$$
-\operatorname{Re}(\mathrm{CO})_{3}\left(2,2^{\prime} \mathrm{Bpy}\right)^{+^{*}}+\mathrm{Amina} \longrightarrow-\operatorname{Re}(\mathrm{CO})_{3}\left(2,2^{\prime} \mathrm{Bpy}\right)^{\bullet}+\mathrm{Amina}^{+\bullet}
$$

En este mecanismo el complejo de Re absorbe luz de longitud de onda, $\lambda: 355$ $\mathrm{nm}$ ó $\lambda: 337 \mathrm{~nm}$ poblando el estado excitado de TCML, que en ausencia de un "desactivador" decae emitiendo luz y en forma no radiativa disipando calor al medio ambiente. Cuando en el medio está presente la Amina, "desactivador", el estado excitado del complejo reacciona con el mismo produciendo el radical reducido $\operatorname{Re}(\mathrm{CO})_{3} \mathrm{Bpy}^{\bullet}$ y la forma oxidada de la amina Amina ${ }^{+\bullet}$. Este proceso está representado por la constante bimolecular kq.

Posteriormente el radical $-\operatorname{Re}(\mathrm{CO})_{3}\left(2,2^{\prime} \mathrm{Bpy}\right)^{\bullet}$ decae con una cinética de primer orden.

\section{Cálculo clásico}

Considerando un mecanismo común para la reacción de transferencia de electrones entre el cromóforo $-\operatorname{Re}(\mathrm{CO})_{3}\left(2,2^{\prime} \mathrm{Bpy}\right)^{+}$, tanto en el monómero como en el polímero, puede plantearse mecanismo descripto en la introducción teórica, ecuación 2::

$$
\mathrm{A}^{+*}+\mathrm{D} \underset{\mathrm{k}_{-\mathrm{d}}}{\stackrel{\mathrm{k}_{\mathrm{d}}}{\rightleftarrows}} \mathrm{A}^{+*}--\mathrm{D} \underset{\mathrm{k}_{-\mathrm{e}}}{\stackrel{\mathrm{k}_{\mathrm{e}}}{\rightleftarrows}} \mathrm{A}--\mathrm{D}^{+} \stackrel{\mathrm{k}_{\mathrm{p}}}{\longrightarrow} \mathrm{A}+\mathrm{D}^{+}
$$

Donde $\mathrm{A}^{+}$representa al cromóforo $-\operatorname{Re}(\mathrm{CO})_{3}\left(2,2^{\prime} \mathrm{Bpy}\right)^{+}$; 
D representa a la amina;

$\mathrm{k}_{\mathrm{d}}$ es la constante de velocidad difusional;

$\mathrm{k}_{-\mathrm{d}}$ es la constante de velocidad de disociación;

$\mathrm{k}_{\mathrm{e}} \mathrm{y} \mathrm{k}_{\mathrm{e}}$ son las constantes de velocidad de transferencia de electrones directa e inversa $\mathrm{y}$

$\mathrm{k}_{\mathrm{p}}$ es la constante de velocidad para la separación del par iónico

Si $k_{p} \gg k_{-e}$, la constante de velocidad global se reduce a la ecuación 10 :

$$
\mathrm{k}_{\mathrm{q}}=\frac{\mathrm{k}_{\mathrm{d}}}{1+\frac{\mathrm{k}_{-\mathrm{d}}}{\mathrm{k}_{\mathrm{e}}}}
$$

Introduciendo el cambio en la energía de Gibbs para el proceso de transferencia de electrones:

$$
k_{e}=k_{e}^{0} \exp \left(-\Delta G^{\mp} / R T\right)
$$

donde, $k_{e}^{0}=\kappa v_{N}$

Para la relación entre $\Delta G^{\mp}$ y $\Delta G^{0}$ se han utilizado la teoría de Marcus ecuación y la teoría de Rehm-Weller, ecuación 42 y 43 respectivamente:

$$
\Delta \mathrm{G}^{\mp}=\Delta \mathrm{G}^{\mp}(0)\left[1+\frac{\Delta \mathrm{G}^{0}}{4 \Delta \mathrm{G}^{\mp}(0)}\right]^{2}
$$




$$
\Delta G^{\mp}=\frac{\Delta G^{0}}{2}+\left[\left(\frac{\Delta G^{0}}{2}\right)^{2}+\Delta G^{\mp}(0)^{2}\right]^{1 / 2}
$$

Con los valores de los radios de las aminas y el radio del complejo $\operatorname{Py} \operatorname{Re}(\mathrm{CO})_{3}\left(2,2^{\prime} \mathrm{Bpy}\right)^{+}$y teniendo en cuenta que para acetonitrilo $\mathrm{n}=1.34$ y $\varepsilon=37.5 \mathrm{a}$ $298 \mathrm{~K}$, se puede estimar la energía de reorganización del solvente $\lambda_{\text {esfera extema }}=0.73 \mathrm{eV}$

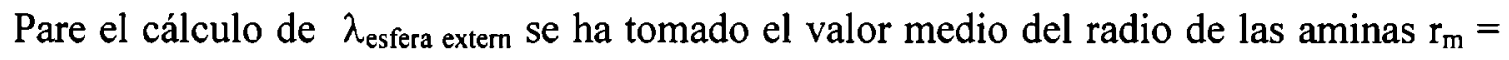
$4.8 \AA^{64}$, y el radio calculado para el complejo $\operatorname{PyRe}(\mathrm{CO})_{3}\left(2,2^{\prime} \mathrm{Bpy}\right)^{+}$, con el programa Hyperchem, $\mathrm{r}_{\mathrm{A}}=7.3 \AA$

La constante de velocidad difusional, $\mathrm{k}_{\mathrm{d}}$, para moléculas sin carga y radios ra $\mathrm{y}$ rm calculada de acuerdo a Smoluchowski da un valor de $2 \times 10^{10} \mathrm{M}^{-1} \mathrm{~s}^{-1}$. Sin embargo en el presente trabajo se ha usado el valor $2.5 \times 10^{10} \mathrm{M}^{-1} \mathrm{~s}^{-1}$ para $\mathrm{kd}$ que es muy similar al valor máximo encontrado para kq. Este valor ha sido utilizado en trabajos previos ${ }^{65}$.

Con las ecuaciones 44 y 45 que surgen de reemplazar ke por su expresión en función del $\Delta G^{\mp} \mathrm{m}$ dada para la teoría de Marcus y la teoría de Rehm-Weller, respectivamente, en la expresión de $\mathrm{kq}$ se realizaron los ajustes de los datos experimentales de kq tanto para el monómero como para el polímero, figura 17.

$$
k_{q}=\frac{k_{d}}{k_{-d}} k_{e}^{0 \exp -\left(\Delta G^{\mp}(0)\left[1+\frac{\Delta G^{0}}{4 \Delta G^{\mp}(0)}\right]^{2}\right) / R T}
$$




$$
k_{q}=\frac{k_{d}}{1+\frac{k_{-d}}{k_{e}^{0} \exp -\left(\frac{\Delta G^{0}}{2}+\left[\left(\frac{\Delta G^{0}}{2}\right)^{2}+\Delta G^{\mp}(0)^{2}\right]^{1 / 2}\right) / R T}}
$$
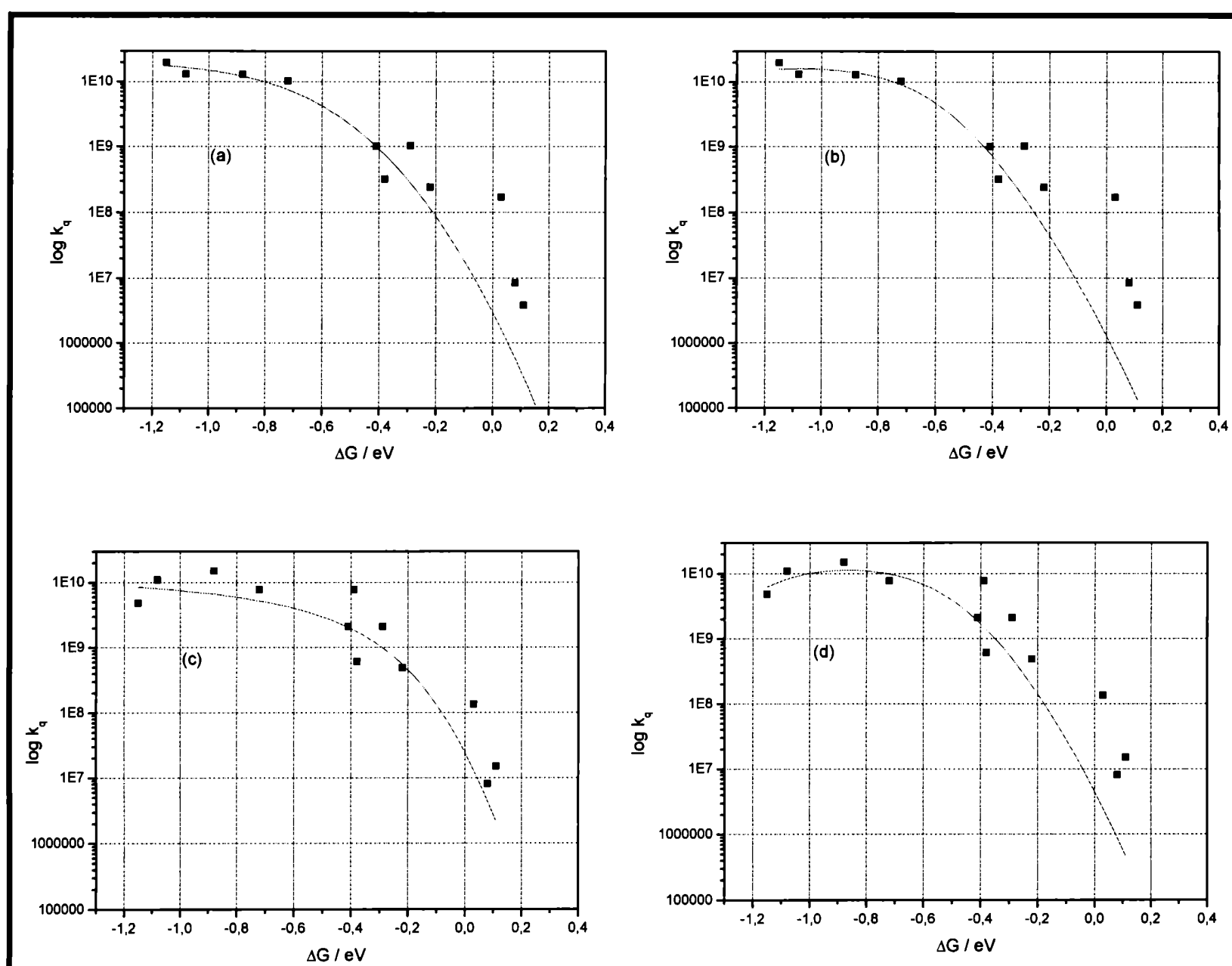

Figura 17: Ajustes de los datos experimentales de log kq versus $\Delta \mathrm{G}$ para (a) $\operatorname{PyRe}(\mathrm{CO}) 3\left(2,2^{\prime} \mathrm{Bpy}\right)$ con la teoría de Rehm-Weller; (b) PyRe(CO)3(2,2'Bpy) con la teoria de Marcus; (c) $\left\{(\mathrm{vpy})_{2} \mathrm{vpyRe}(\mathrm{CO})_{3}\left(2,2^{\prime} \mathrm{Bpy}\right)^{+}\right\}_{\mathrm{n}-200}$ con la teoría de Rehm-Weller y (d) $\left\{(\mathrm{vpy})_{2} \mathrm{vpyRe}(\mathrm{CO})_{3}\left(2,2^{\prime} \mathrm{Bpy}\right)^{+}\right\}_{\mathfrak{n} 200}$ con la teoría de Marcus 
Los parámetros, $\Delta G^{\mp}(0)$, y $k_{e}^{0}$ que resultan de los ajustes de los datos experimentales de quenching del polímero y el monómero se muestran en la tabla 2.

\begin{tabular}{|l|l|l|l|l|}
\hline \multirow{2}{*}{} & \multicolumn{2}{|c|}{ Teoría de Marcus } & \multicolumn{2}{c|}{ Teoría de Rehm-Weller } \\
\cline { 2 - 5 } & $\Delta \mathrm{G}^{*}(0) / \mathrm{eV}$ & $k_{e}^{0} / \mathrm{s}^{-1}$ & $\Delta \mathrm{G}^{*}(0) / \mathrm{eV}$ & $k_{e}^{0} / \mathrm{s}^{-1}$ \\
\hline PyRe(CO $)_{3}\left(2,2^{\prime} \mathrm{Bpy}\right)^{+}$ & 0.26914 & $8.4 \times 10^{9}$ & 0.3575 & $6.2 \times 10^{11}$ \\
\hline$\left\{(\mathrm{vpy})_{2} \mathrm{vpy}^{\mathrm{N}}(\mathrm{CO})_{3}\left(2,2^{\prime} \mathrm{Bpy}\right)^{+}\right\}_{\mathrm{n}-200}$ & 0.21649 & $3.8 \times 10^{9}$ & 0.19264 & $8.1 \times 10^{9}$ \\
\hline
\end{tabular}

Tabla 2: Parámetros $\Delta \mathrm{G}^{*}(0)$ y $k_{e}^{0}$ para el polímero $\left\{(\mathrm{vpy})_{2} \mathrm{vpyRe}(\mathrm{CO})_{3}\left(2,2^{\prime} \mathrm{Bpy}\right)^{+}\right\}_{\mathrm{n}-200}$ y el monómero $\operatorname{PyRe}(\mathrm{CO})_{3}\left(2,2^{\prime} \mathrm{Bpy}\right)^{+}$obtenidos de los ajustes según la teoría de Marcus y la teoría de RehmWeller

El valor de $\Delta G^{\mp}(0)$ que se obtiene del ajuste según la teoría de Marcus, es menor para el polímero que para el monómero. Según la teoría de Marcus $\Delta G^{\mp}(0)=\lambda /$ 4, como se mencionó en la parte teórica $\lambda$ representa la energía de reorganización y está representada por dos contribuciones, una energía de reorganización de esfera interna, y una energía de reorganización de esfera externa.

En trabajos anteriores ${ }^{66}$ se ha observado con microscopía electrónica de transmisión, TEM, y dispersion de luz estática y dinámica, que el polímero $\left\{(\mathrm{vpy})_{2} \mathrm{vpyRe}(\mathrm{CO})_{3}\left(2,2^{\prime} \mathrm{Bpy}\right)^{+}\right\}_{200}$ forma micelas cuando se obtienen los films por evaporación del solvente acetonitrilo.

Si bien la teoría de Marcus ha sido desarrollada para sistemas en soluciones homogéneas; esta teoría ha sido aplicada a sistemas micelares ${ }^{67,68}$. Los movimientos de las moléculas de solvente están restringidos en un medio micelar, varios órdenes de 
magnitud comparados con un medio homogéneo. Por lo tanto la reorganización del solvente puede no contribuir completamente a la energía de reorganización exerna, en la escala de tiempo en la que se produce la transferencia de electrones entre el estado excitado de TCML del polímero y las aminas. Además la energía de reorganización interna para la transferencia de electrones, puede disminuir en el polímero, vía modos vibracionales de las piridinas no complejadas. Estos hechos, harían que $\Delta G^{\mp}(0)$ para la transferencia de electrones sea menor en el polímero con respecto al monómero.

Para el ajuste según la teoría de Rehm-Weller, se observa también que $\Delta \mathrm{G}^{*}(0)$ es menor en el polímero que en el monómero.

Además en todos los casos, el valor de $k_{e}^{0}$ que se obtiene del ajuste de ambos sistemas con amabs teorías, es menor que el valor límite esperado para una reacción de transferencia electrónica adiabática. Esta disminución puede ser entendida, si se introduce cierto grado de no adiabaticidad a la reacción ${ }^{69}$. Ha sido propuesto un carácter no-adiabático para el quenching del triplete de hidrocarburos aromáticos por $\mathrm{Cu}(\mathrm{II})$ $\operatorname{bis}(2,4 \text { pentadionato })^{70}$.

Como se ha visto en la parte teórica según la teoría de transición clásica la constante de primer orden $k_{e}^{0}$ está dada por:

$$
k_{e}^{o}=\kappa_{e l} \nu_{N}
$$

Donde $\kappa_{e l}$ es el coeficiente de transmisión electrónica y $v_{N}$ es la frecuencia nuclear para el pasaje de estados de transición $\left(v_{N}=10^{13} \mathrm{~s}^{-1}\right)$ con $\kappa_{e l}=1$ en el límite clásico. 
De la teoría de mecánica cuántica se obtiene la ecuación:

$$
k_{e}^{0}=\frac{H_{r p}^{2}}{h}\left(\frac{\pi}{\lambda R T}\right)^{1 / 2}
$$

Donde $H_{r p}$ es la matriz de acoplamiento electrónico entre reactivos y productos y $\lambda$ la energía de reorganización. La matriz elemental para el acoplamiento electrónico está dada por:

$$
H_{r p}=\left(\Psi_{R}\left|H_{e l}\right| \Psi_{P}\right)
$$

Donde $\Psi_{R}$ y $\Psi_{P}$ son las funciones de onda electrónica de equilibrio para reactivos y productos y $\mathrm{H}_{\mathrm{el}}$ es el Hamiltoniano electrónico. La función de onda electrónica total debe incluir las funciones de onda espacial y de spin. Por lo tanto la matriz electrónica elemental puede ser factorizada en un factor espacial y un factor de spin. El factor de spin es diferente de cero para estados de igual multiplicidad. Este grado de aproximación no es válido para reacciones de estado triplete, debido a la contribución de estados de difernete multiplicidad a la función de onda de los productos. En este caso se espera un valor mas bajo para Hrp, lo cual resulta en un valor mas bajo de $k_{e}^{0}$.

La dispersidad observada, puede estar relacionada, en parte, con la diferencia en la energía de reorganización interna para cada una de las aminas. 


\section{Referencias}

1. H. Taube, Electron Transfer Reactions of Complex Ions in Solution, Academic Press, New York, 1970.

2. R.D. Cannon, Electron Transfer Reactions, Butterworths, London, 1980.

3. (a) R.A. Marcus, Discuss. Faraday Soc. 29, 1960 21; (b) Annu. Rev. Phys. Chem. 15, 1964 155; (c) J. Chem. Phys. 43, 1965, 679; (d) Electrochim. Acta 13, $1968, \mathrm{p} 995$.

4. N.S. Hush Trans. Faraday Soc. 57, 1961, p 155.

5. (a) M.A. Vorotyntsev, R.R. Dogonadze and A.M. Kuznetsov, Dokl. Akad. Nauk SSSR 195, 1970 1135; (b) E.D. German, V.G. Dvali, R.R. Dogonadze and A.M. Kuznetsov Elektrokimiya 12, 1976, 639; (c) R.R. Dogonadze and A.M. Kuznetsov, Prog. Surf. Sci. 6 (1975) 1, Elektrokimiya 2, 1967, 1324; (d) R.R. Dogonadze, A.M. Kuznetsov and V.G. Levich Electrochim. Acta 13, 1968, 1025; (e) V.G. Levich Adv. Electrochem. Eng. 4, 1966, p 249.

6. W.F. Libby, J. Phys. Chem. 56, 1952, p 863.

7. R.A. Marcus, B.J. Zwolinski and H. Eyring J. Phys. Chem. 58, 1954, p 432.

8. J. Weiss Proc. Roy. Soc. (London) 222, 1954, p 128.

9. B.J. Zwolinski, R.A. Marcus and H. Eyring Chem. Rev. 55, 1955, p 157.

10. R.A. Marcus J. Chem. Phys. 24, 1956, p 966.

11. R.A. Marcus J. Chem. Phys. 26, 1957, p 867.

12. R.A. Marcus, Office of Naval Research, Project No. NR 051-339, 1957, published in Special Topics in Electrochemistry, P.A. Rock, Ed., Elsevier, New York, 1977, p. 180; cf. Can. J. Chem. 37, 1959, p 155.

13. R.L. Platzman and J. Franck, L. Farkas Memorial Volume, A. Farkas and E.P. Wigner, Eds., Research Council of Israel, Jerusalem, Special Publication No. 1, p. $21,1952$.

14. R.L. Platzman and J. Franck, Z. Phys. 138, 1954, p 411.

15. R.A. Marcus, Trans. N. Y. Acad. Sci. 19, 1957, p 423.

16. N. Sutin, Ann. Rev. Nucl. Sci. 12, 1962, p 285.

17. V.G. Levich and R.R. Dogonazde Dokl. Akad. Nauk SSSR 124, 1959, p 123.

18. V.G. Levich and R.R. Dogonazde Dokl. Akad. Nauk SSSR 133, 1960, p 158. 
19. R.R. Dogonadze Dokl. Akad. Nauk SSSR 133, 1960, p 1368.

20. R.R. Dogonadze Dokl. Akad. Nauk SSSR 142, 1960, p 1108.

21. R.R. Dogonadze Reactions of Molecules at Electrodes, N.S. Hush, Ed., Wiley, New York, 1971; Chapter 3, p. 135.

22. R.R. Dogonadze, J. Ulstrup and Yu. I. Kharkats J. Chem. Soc. Faraday Trans. $68,1972, \mathrm{p} 744$.

23. S. Efrima and M. Bixon J. Chem. Phys. 64, 1976, p 3639.

24. S. Efrima and M. Bixon Chem. Phys. 13, 1976, p 445.

25. N.R. Kestner, J. Logan and J. Jortner J. Phys. Chem. 78, 1974, p 2148.

26. S.B. Piepho, E.R. Krausz and P.N. Schatz J. Am. Chem. Soc. 100, 1978, p 2996.

27. P.P.Schmidt Australian J. Chem. 23, 1970, p 1287.

28. M.A. Vorotyntsev, R.R. Dogonazde and A.M. Kuznetsov Phys. Stat. Solidi 54, 1972, p 125.

29. M.A. Vorotyntsev, R.R. Dogonazde and A.M. Kuznetsov Phys. Stat. Solidi 54 1972, p 425.

30. M.J. Weaver and E.L. Yee Inorg. Chem. 19, 1980, p 1936.

31. M.D. Newton Int. J. Quant. Chem. Symp. 14. 1980, p 363.

32. M.D. Newton ACS Symp. Series No. 198, 1982, p 255.

33. J. Ulstrup and J. Jortner J. Chem. Phys. 63, 1975, p 4358.

34. R.Ballardini, G. Varani, M.T. Indelli, F. Scandola and V. Balzani J. Am. Chem. Soc. 100, 1978, p 7219.

35. J. V. Beitz and J. R. Miller J. Chem. Phys. 71, 1979, p 4579.

36. C. R. Bock, J. A. Connor, A. R. Gutierrez, T. J. Meyer, D. G. Whitten, B.P. Sullivan and J. K. Nagle J. Am. Chem. Soc. 101, 1979, p 4815.

37. C. Creutz and N. Sutin J. Am. Chem. Soc. 99, 1977, p 241.

38. D. Rehm and A. Weller Israel J. Chem. 8, 1970, p 259.

39. S. Efrima and M. Bixon Chem. Phys. Lett. 25, 1974, p 34.

40. R.A. Marcus Int. J. Chem. Kin. 13, 1981, p 865.

41. P. Siders and R.A. Marcus J. Am. Chem. Soc. 103, 1981, 748-752.

42. R. P. Van Duyne and S.F. Fischer Chem. Phys. 5, 1974, p 183.

43. D. Rehm and A. Weller Ber. Bunsenges. Phys. Chem. 73, 1969, p 834. 
44. (a) N. Mataga and N. Nakashima Spectrosc. Lett. 8, 1975, p 275, (b) H. Masuhara and N. Mataga Acc. Chem. Res.14, 1981, p 312.

45. H.D. Gafney and A.W. Adamson J. Am. Chem. Soc. 94, 1972, p 8238.

46. V. Balzani, F. Bolleta, M.T. Gandolfi and M. Maestri Top. Curr. Chem. 75, 1978, p 1 .

47. N. Sutin and C. Creutz Pure Appl. Chem. 52, 1980, p 2717.

48. N. Sutin J. Photochem. 10, 1979, p 19.

49. C.R. Bock, T. J. Meyer and D. G. Whitten J. Am. Chem. Soc. 96, 1974, p 4710.

50. (a) C. P. Anderson, D.J. Salmon, T.J. Meyer and R.C. Young J. Am. Chem. Soc. 99, 1977, p 1980; (b) M. Maestri and M. Grätzel, Ber. Bunsenges. Phys. Chem. 81, 1977. p 504.

51. T. J. Meyer Acc. Chem. Res. 11, 1978, p 94.

52. F. Scandola and V. Balzani J. Chem. Ed. 60, 1983, p 814.

53. N.S. Hush Electrochim. Acta 13, 1968, p 1005.

54. N. Sutin Progr. Inorg. Chem. 30, 1983441.

55. R.A. Marcus and N. Sutin Biochim. Biophys. Acta 811, 1985, p 265.

56. R.A. Marcus J. Chem. Phys. 43, 1965, 679-601.

57. R.A. Marcus, Electron transfer reactions, in: H. Hartman Ed., Chemische Elementarprozesse, Springer-Verlag, 1968, pp. 348-356.

58. R.A. Marcus, Electron transfer and tunneling in chemical and biologycal systems, in: B. Chance, D. DeVault, H. Frauenfelder, R.A. Marcus, J.R. Schrieffer and N. Sutin (Eds.), Tunneling in Biologycal Systems, Academic Press, New York, 1979, pp. 109-127.

59. R.A. Marcus, Electron transfer and tunneling in chemical and biologycal systems, in: H. Gerischer and J.J. Katz (Eds.), Light-Induced Charge Separation in Biology and Chemistry, Verlag-Chemie, Berlin, 1979, pp. 15-43.

60. N. Mataga, Photochemycal Energy Conversion (Eds. J.R. Norris Jr. and D. Meisel), Elsevier, Amsterdam, 1989, p. 32.

61. V. Balzani and F. Scandola: Energy Resources through Photochemistry and Catalysis (Ed. M. Graetzel), Academic, London, 1983, p. 1.

62. Wolcan, E. And Félia, M. Photochem. Photobiol. Sci, 2003,2,412-417 
63. L. Sacksteder; A. Zipp; E. A. Brown; J. Streich; J. N. Demas and B. A DeGraff. Inorg. Chem. 1990, 29, 4335-4340.

64. Tesis docotoral de E. Wolcan, Universidad Nacional de La Plata. 1995.

65. T.M. McCleskey, J.R. Winkler, H.B. Gray, J. Am. Chem. Soc. 114, 1992, 6935

65. E. Wolcan; J. L. Alessandrini; and M. R. Féliz. J. Phys. Chem. B, 2005, 1091 22890-22898.

66. H. L. Tavernier; A. V. Barzykin; M. Tachiya; M. Fayer. J. Phys. Chem. B, 1998, 102,6078 .

67. M. Kumbhakar; S. Nath; T. Mukherjee; H. Pal. J. Chem. Phys. 2004, 120, 2824.

68. G. Porcal; G. Bertolotti; C. M. Previtali; and M. V. Encinas. Chem. Phys. 2003, $5,4123-4128$.

69. G. L. Hug; and B. Marciniak. J. Phys. Chem. 1994, 98, 7523-7532. 